NOVEMBER 2019

VOLUME 10 ISSUE S1
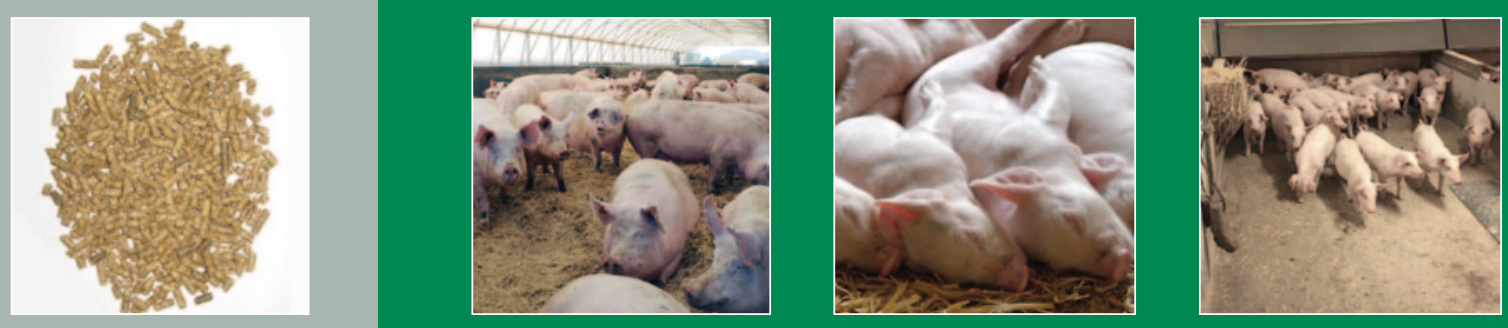

\title{
Advances in Animal Biosciences
}

\section{Manipulating Pig Production XVII}

Proceedings of the Seventeenth Biennial Conference of the Australasian Pig Science Association (APSA)

Adelaide, Australia

17-20 November 2019 


\section{Advances in Animal Biosciences}

\section{Management Board}

Nigel Scollan (chair), Maggie Mitchell, Eileen Wall, Howard Simmins (BSAS); Philippe Chemineau, Matthias Gauly, Andrea Rosati (EAAP); Nicolas Friggens, Stephane Ingrand, Odile Hologne (INRA)

\section{Editor-in-Chief}

Cledwyn Thomas

\section{Aims and Scope}

Advances in Animal Biosciences is an associated publication to the journal animal. It aims to publish highquality conference, symposium and workshop proceedings about animal-related aspects of the life sciences with emphasis on farmed and other managed animals. These can be in the form of a book of abstracts, summaries or complete papers. The format will highlight the title of the meeting and organisations involved but the publications will have the added advantage of forming a series under Advances in Animal Biosciences.

Subject areas can include aspects of Breeding and Genetics, Nutrition, Physiology and Functional Biology of Systems, Behaviour, Health and Welfare, Livestock Farming Systems, Human Health and Product Quality.

However, due to the integrative nature of biological systems, monographs and conference proceedings dealing with the translation of basic and strategic science into the whole animal and farming system and the impact on Productivity, Product Quality, Food Security, the Environment, Climate Change and Humans will be particularly welcome.

\section{Information for Conference Organisers}

The Animal Consortium together with Cambridge University Press offers conference organisers a package that enables publication of high-quality conference, symposium and workshop proceedings about animal-related aspects of the life sciences with emphasis on farmed and other managed animals.

Summaries, abstracts or full papers may be published in Advances in Animal Biosciences and high-quality invited papers from these meetings may be submitted and published as a defined series in animal.

Conference organisers interested in publishing their proceedings should send an outline proposal for publication in Advances in Animal Biosciences, animal, or both journals to cko @ cambridge.org. The publisher together with the Editors-in-Chief will then provide an estimate of costs and the procedures to be used.

Manuscripts submitted to Advances in Animal Biosciences will be reviewed by the Editor-in-Chief, supported by guest editors and papers submitted to animal will be peer reviewed. If accepted after review, proceedings will be published within 12 weeks of receipt by the Publisher.

Advances in Animal Biosciences and animal are titles included on Cambridge Core which can be found at: www.cambridge.org/ABS and www.cambridge.org/anm, respectively.

Further information on these and on other Press titles can be found at www.cambridge.org/core. 


\section{Manipulating Pig Production XVII}

Proceedings of the Seventeenth Biennial Conference of the Australasian Pig Science Association (APSA)

Adelaide, Australia

17-20 November 2019

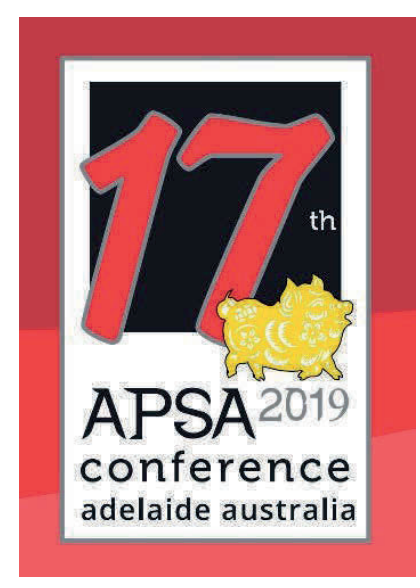

Edited by Dr Kate Plush and Mr Robert Hewitt AUSTRALASIAN PIG SCIENCE ASSOCIATION (INC.) 
Manipulating Pig Production XVII comprises extended abstracts presented at the Seventeenth Biennial Conference of the Australasian Pig Science Association held in Adelaide, 17-20 November 2019.

The extended abstracts were peer-reviewed and edited to maintain the scientific standards set by the APSA Committee. Abstracts not meeting these standards were rejected and have not been included in the proceedings. Views expressed in all contributions are those of the authors and not those of APSA.

(C) Copyright 2019 by the Australasian Pig Science Association (Inc.) Viveash, WA 6056, Australia. ISSN: 2040-4700 


\section{Contents}

page

Preliminary Material

i - xviii

Summaries

s01 -s99

Author Index

I - II 


\section{Acknowledgements}

The Australasian Pig Science Association (APSA) was established in 1987 to provide a forum for excellence in science related to all aspects of the pig industry. APSA hosts the APSA conference every two years and in 2019, the 17th APSA conference will be held. Since its inception, the APSA conference has continued to grow both in size and in recognition for the quality of papers presented. The success of APSA and the APSA conference may still be attributed to the dedication and foresight of the original founding Committee members for which we remain grateful.

One of the priorities of the APSA conference is to provide the opportunity for new research scientists and students to be able to present their work and foster collaboration with the pig science community. We are pleased to be able to continue with this tradition of supporting new scientists and I wish them a warm welcome to our community.

The APSA conference is well known for its invited speakers and at this conference we are pleased to host Dean Boyd, Carol Bagnell, Kristy DiGiacomo, Mark Hutchinson, Clay Lents, Steve Little, Tomas Norton, Chris Piotrowski, Mike Tokach and Robyn Warner. On behalf of the organising Committee I would like to thank them each for their contributions. The 17th APSA conference brings a new chapter for the publication of the conference proceedings and for the first time, we have partnered with Advances in Animal Biosciences and animal to increase the exposure of the submitted papers and provide greater recognition to the contributing authors. To our Editors, Kate Plush, Robert Hewitt and John Patience, thank you for your work in coordinating the publication of our invited and contributed papers.

Of course, the conference would not be possible without the work of a small but dedicated team of people. I would like to thank the organising Committee who have worked tirelessly and were always willing to assist where matters arose. Accordingly, thanks go to Pat Mitchell (Immediate Past President), Robert Hewitt (Vice President), Frank Dunshea (Treasurer), Cameron Ralph (Secretary), Rebecca Athorn, John Pluske, Charlie Rikard-Bell and Robyn Terry. A big thank you also to Emalyn Loudon who has undertaken the role of Executive Manager for APSA. I would also like to acknowledge the hard work of our conference Secretariat, Kate Murphy and her team at YRD who continue to assist the organising Committee to produce a world-leading pig science conference.

On behalf of the APSA conference Committee, I would like to acknowledge all the conference sponsors, which without their support, the APSA conference would not be possible. Special mention should be given to Australian Pork Limited (APL) and Australasian Pork Research Institute Limited (APRIL) who are joint principal sponsors of APSA 2019. To our Gold, Silver, Bronze and supporting partners I also offer my thanks for your continued support of our great APSA conference.

Finally, it has been a great pleasure to serve as APSA President for the past two years. I am incredibly grateful and humbled to have been able to work with so many dedicated and supportive people. Thank you for your support of APSA and to the delegates attending the 17th APSA conference, I wish you all a happy and productive conference.

\section{Dr Stuart Wilkinson}

President 


\title{
APSA Awards
}

\section{The APSA Fellow Award}

The APSA Fellow Award was first presented in 2007. This prestigious award is offered in recognition of past and present members who have made an outstanding contribution to APSA as well as their contribution and commitment to pig science. Nominations for the APSA Fellow Award are received from the current APSA Committee and the initial nominations are supported by a verbal statement addressing the selection criteria, weighted to assist the Committee in its deliberations. The selection criteria for the deliberation of APSA Fellow nominations cover contributions to:

- The APSA Committee (President, Vice-President, Secretary, Treasurer, ordinary Committee member)

- Technical content contribution (Editor, referee, scientific authors, etc.)

- Membership development (students, national and overseas)

- Contribution and commitment to Pig Science (research, involvement in post-graduate training)

All past APSA fellows were significant contributors to APSA and pig science, and given both their professional and personal commitments at the time, their substantial contributions are more utterly astonishing and valued.

Previous recipients:

\author{
Dr Ray King (2007) \\ Dr David Hennessy (2007) \\ Dr Michael Taverner (2009) \\ Dr Ian Williams (2011) \\ Professor Frank Dunshea (2013) \\ Dr Bruce Mullan (2013) \\ Professor John Pluske (2015) \\ Dr Robert van Barneveld (2017)
}

\section{The Batterham Memorial Award}

The Batterham Memorial Award is a prestigious award conferred by APSA in memory of the late Dr Ted Batterham. Ted Batterham's love of pigs began at the NSW Agriculture, Wollongbar Research Station in the mid-1960s when he began work with Dr John Holder to solve the problem of variability in the growth of pig fed meat meals. At that time abattoirs in NSW produced meat meals that were very variable because there was little control on either the raw materials used or cooking times and temperatures. Ted soon realised that part of the variability was explained by the content of bone but, something much more fundamental that would keep Ted focussed and fascinated for the rest of his professional life, was the variability of available lysine in these meals. Ted knew that if proteins were heated in the presence of carbohydrates and fats, lysine would become unavailable to the pigs own enzymes.

Ted went to The University of Melbourne to commence a PhD with Tony Dunkin to develop an in vivo assay in rats and pigs to quantify the available lysine not just on meat meals but in a range of other protein sources and cereals. He returned to Wollongbar and became a world leader in the availability of amino acids in feedstuffs for pigs and poultry. Not content just to solve a problem, Ted wanted to find solutions and reasoned that, if the availability of lysine was known, any shortfall could be remedied by supplementation with synthetic lysine. That idea stimulated research that delved into ways that the biological value of proteins could be enhanced by supplementation with synthetic amino acids.

Ted's research career was always focused on industry issues and driven by a desire to find suitable solutions. He knew that progress was best made by teams of people stimulating and supporting each other, and that investment in young people was essential.

Therefore, the Batterham Memorial Award is made to a young scientist, a person within 10 years of graduation. Its aim is to 'stimulate and develop innovation in the pig industry'. It is anticipated that the cash award will enable the recipient to broaden their exposure to national or international pig science. Previous winners of the Batterham Memorial Award:

Robert van Barneveld (1995)

John Pluske (1997)

Kaye Coates (1999)

Darryl D'Souza (2001)

Patricia Mitchell (2003)

Eva Ostrowska (2005)
David Cadogan (2007)

Rebecca Morrison (2009)

Cherie Collins (2011)

Robert Smits (2013)

Heather Channon (2015)

Kate Plush (2017) 


\section{APSA - Behind the scenes}

APSA has remained a successful and relevant Association through the dedication and commitment of the elected Committees since 1987 . The following contributions are gratefully acknowledged by the Australasian pig science community.

1987

President
Ray King
Vice President
Paul Hemsworth

1989

1991

1993

1995

\section{President \\ Ray King \\ Vice President \\ Paul Hemsworth}

President

Paul Hemsworth

Vice President

Mike Taverner

Past President

Ray King

President

Mike Taverner

Vice President

Bruce Mullan

Past President

Paul Hemsworth

President

Bruce Mullan

Vice President

Andy Paterson

Past President

Mike Taverner

President

Frank Dunshea

Vice President

Robert van Barneveld

Past President

Bruce Mullan

President

Robert van Barneveld

Vice President

Neil Gannon

Past President

Frank Dunshea

\section{President}

Ian Williams

Vice President

John Pluske

Past President

Robert van Barneveld

Secretary
David Hennessy
Treasurer
Greg Cronin

Committee

John Barnett

Ted Batterham

Chris Hansen

Paul Hughes

Noel Johnston

Secretary
David Hennessy
Treasurer
Greg Cronin

Secretary
David Hennessy
Treasurer

Greg Cronin

\section{Secretary}

David Hennessy

Treasurer

Greg Cronin

Secretary

Dean Revell

Treasurer

Rob Smits

Secretary

Neil Gannon

Treasurer

Rob Smits

Secretary

Colin Cargill

Treasurer

Heather Bray

Secretary

Sally Tritton

Treasurer

Rob Smits
Committee

John Barnett

Ted Batterham

Chris Hansen

Paul Hughes

Noel Johnston

Committee

Ross Cutler

Ted Batterham

Bruce Mullan

Roger Campbell

Peter Cranwell

Chris Hansen

Committee

Robert Love

Ross Cutler

Peter Cranwell

Ted Batterham

\section{Committee}

Paul Hartmann

David Hampson

Sue Skirrow

Frank Dunshea

Committee

Robert Love

Tony Peacock

Robyn Warner

Ian Williams

Committee

John Pluske

Susanne Hermesch

Ian Williams

John Hargraves

\section{Committee}

Susanne Hermesch

Mingan Choct

Darryl D'Souza

Ray King

Mike Taverner
Editor

APSA Committee

\section{Editors}

John Barnett

David Hennessy

Editor

Ted Batterham

\section{Editor}

Ted Batterham

Editors

David Hennessy

Peter Cranwell

Editor

Peter Cranwell

Editor

Peter Cranwell

Editor

Janet Paterson 
Past President

Ian Williams

2005

President

Pat Mitchell

Vice President

Bruce Mullan

Past President

John Pluske

2007

President

Bruce Mullan

Vice President

Neil Gannon

Past President

Pat Mitchell

2009

President

Neil Gannon

Vice President

Darryl D'Souza

Past President

Bruce Mullan

2011

President

Darryl D'Souza

Vice President

David Cadogan

Past President

Neil Gannon

2013

President

David Cadogan

Vice President

Cherie Collins

Past President

Darryl D'Souza

2015

President

Cherie Collins

Vice President

Alison Collins

Past President

David Cadogan

President

Pat Mitchell

Vice President

Stuart Wilkinson

Past President

Cherie Collins

President

Stuart Wilkinson

Vice President

Robert Hewitt

Past President

Pat Mitchell
Susanne Hermesch

Ray King

Geogy Philip

Secretary

Ian Barugh

Treasurer

Committee

Frank Dunshea

Geogy Philip

Graham Pearson

Patrick Morel

Rob Smits

\section{Secretary}

Hugh Payne

Karen Moore

Treasurer

Megan Trezona

Committee

Frank Dunshea

Heather Channon

Rob Smits

Darryl D’Souza

\section{Secretary}

Karen Moore

Treasurer

Megan Trezona

Committee

Frank Dunshea

Heather Channon

Brian Luxford

Trish Holyoake

Secretary

Karen Moore

Treasurer

Megan Trezona

Secretary

Amy Lealiifano

Treasurer

Megan Trezona

Secretary

Amy Lealiifano

Treasurer

Emalyn Loudon

Secretary

Cameron Ralph

Treasurer

Frank Dunshea

Secretary

Cameron Ralph

Treasurer

Frank Dunshea
Committee

John Pluske

Cherie Collins

Heather Channon

Trish Holyoake

\section{Committee}

John Pluske

Alison Collins

Jae Kim

Emalyn Loudon

\section{Committee}

Frank Dunshea

Jae Kim

Pat Mitchell

John Pluske

Committee

Robert Hewitt

John Pluske

Charles Rikard-Bell

Robyn Terry

Committee

Rebecca Athorn

John Pluske

Charles Rikard-Bell

Robyn Terry
Editor

Janet Paterson

Editors

Janet Paterson

Jenny Barker

Editor

Robert van Barneveld

\section{Editor}

Robert van Barneveld

\section{Editors}

John Pluske

Jo Pluske

Editors

John Pluske

Jo Pluske

Editors

Lucy Waldron

Shay Hill

Editors

Kate Plush

Robert Hewitt

Executive Manager

Emalyn Loudon 


\section{APSA XVII Supporters}

The APSA Committee would like to acknowledge the following sponsors for their continued support.

Principal Sponsors

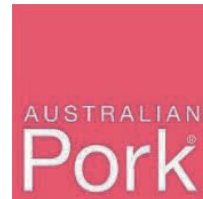

Australasian

Pork Research

Institute Ltd

APRIL

Diamond Sponsor

\section{DSM}

BRIGHT SCIENCE. BRIGHTER LIVING.

Gold Sponsors

W-BASF
We create chemisty

Silver Sponsors

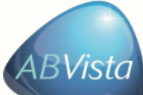
RESEARCH AND
A R D I SOUTH AUSTRALAN
DEVELPENT INSTITUTE
PHYTÓBIOTICS
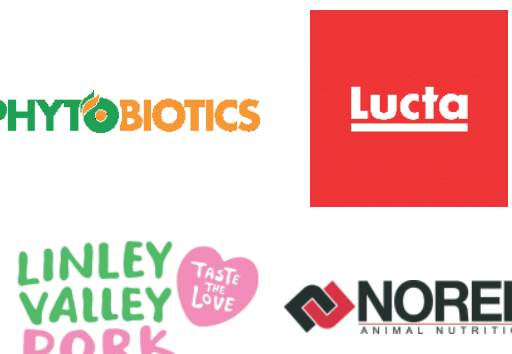

NOREL

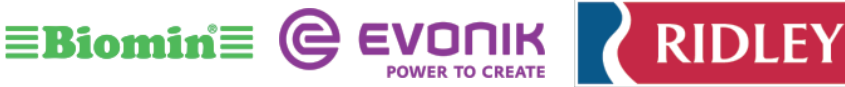

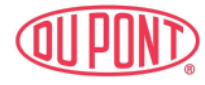

Danisco

Animal Nutrition
Bronze Sponsors

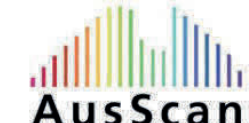

Aus Scan 'I|||'י11|||||||||'

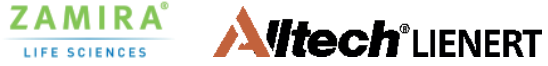

Coffee Cart

Award Sponsors

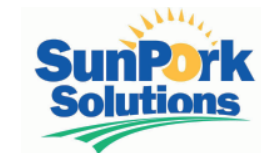

Pen and Notebook

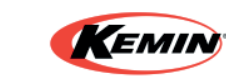

INSPIREO MOLECULAR SOLUTIONS",
Lanyard

\section{LALLEMAND}

LALLEMAND ANIMAL NUTRITION
Meat Suppliers

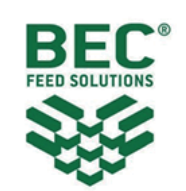

Rivalea zoetis

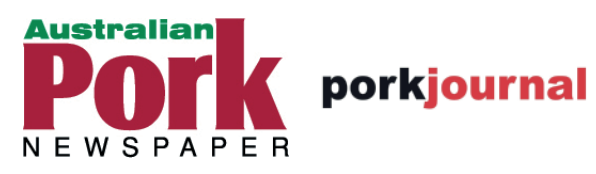




\section{List of contributors}

Abbott L K

Abelilla J J

Abraham S

Aitken R

Alexopoulos J G

Allerson M W

Ao X

Artuso-Ponte V

Ashman $\mathrm{H}$

Athorn R Z

Bacou E

Ball R O

Ballares A

Barbosa F F

Barnes T S

Barton M D

Bedford M R

Beer S L

Bell A W

Biggs L

Billman-Jacobe $\mathrm{H}$

Bittner E P

Blackall P J

Bortolozzo F P

Botella J

Braden C J

Brewster C J

Browne N

Bruneel B

Buchanan E S

Bunter K L

Bunz A M G

Cadogan D J

Calderon H I

Campbell R G

Canal B

Cantarelli V

Celi P

Channon H A

Chaves R

Chen $\mathrm{C}$

Chen D-W

Cheng L

Cho H M

Chuck G

Clarke T

Cochran Z

Cockshott I

Condous P C

Cordero G
The University of Western Australia, Crawley, WA 6009, Australia

University of Illinois at Urbana-Champaign, Champaign 61801, USA

Murdoch University, Murdoch, WA 6150, Australia

Rivalea Australia Pty Ltd, Corowa, NSW 2646, Australia

SunPork Group, Murarrie, QLD 4172, Australia

Holden Farms, Inc., Northfield, MN 55057, USA

Dankook University, Cheonan 31116, Republic of Korea

Phytobiotics Futterzusatzstoffe GmbH, 65343 Eltville, Germany

The University of Melbourne, Parkville, VIC 3010, Australia

Australian Pork Limited, Barton, ACT 2600, Australia

DSM Nutritional Products France, 68128 Village-Neuf, France

University of Alberta, Edmonton, AB T6G 2R3, Canada

Peel Pork, Yarloop, WA 6218, Australia

EW Nutrition GmbH, 49429 Visbek, Germany

The University of Queensland, Gatton, QLD 4343, Australia

University of South Australia, Adelaide, SA 5000, Australia

AB Vista, Marlborough, Wiltshire SN8 4AN, UK

Rivalea Australia Pty Ltd, Corowa, NSW 2646, Australia

Cornell University, Ithaca, NY 14850, USA

Integrity Ag \& Environment, Highfields, QLD 4352, Australia

The University of Melbourne, Parkville, VIC 3010, Australia

The University of Melbourne, Parkville, VIC 3010, Australia

QAAFI, The University of Queensland, St Lucia, QLD 4067, Australia

Federal University of Rio Grande do Sul, Porto Alegre, RS 90040-060, Brazil

The University of Queensland, St Lucia, QLD 4067, Australia

Rivalea Australia Pty Ltd, Corowa, NSW 2646, Australia

Rivalea Australia Pty Ltd, Corowa, NSW 2646, Australia

Alltech Bioscience Centre, Dunboyne, Meath A86, Ireland

Orffa Additives BV, 4251 LC Werkendam, the Netherlands

University of Minnesota, Morris, MN 56267, USA

Animal Genetics and Breeding Unit, University of New England, Armidale, NSW 2351, Australia

Rivalea Australia Pty Ltd, Corowa, NSW 2646, Australia

Feedworks Pty Ltd, Romsey, VIC 3434, Australia

Kansas State University, Manhattan, KS 66506, USA

RG Campbell Advisory, Semaphore, SA 5019, Australia

NOREL S.A., Madrid 28007, Spain

Universidade Federal de Lavras, Lavras, MG 37200-000, Brazil

DSM Nutritional Products USA, Parsippany, NJ 07054, USA

Australian Pork Limited, Barton, ACT 2600, Australia

Universidade Federal de Lavras, Lavras, MG 37200-000, Brazil

Jiangsu University, Zhenjiang 212013, Jiangsu, People's Republic of China

Sichuan Agricultural University, Yaan, Sichuan 625014, People's Republic of China

Dankook University, Cheonan 31116, Republic of Korea

Chungnam University, Daejeon 34134, Republic of Korea

Apiam Animal Health Limited, East Bendigo, VIC 3550, Australia

Westpork Pty Ltd, Maylands, WA 6051, Australia

Cool Sparky, Stoneville, WA 6081, Australia

ANIMINE, 74960 Meythet, Annecy, France

SunPork Group, Murarrie, QLD 4172, Australia

AB Vista, Marlborough, Wiltshire SN8 4AN, UK 
Corso A C

Cottrell J J

Craig J R

D'Souza D N

de Groot N

De Luca V

de Ruyter E M

Degroote J

dela Cruz A R

Della Pia E A

Delles R M

DeRouchey J M

Doyle R E

Dritz S S

Dunlop R H

Dunshea F R

Dutton S

Edwards A C

Edwards L E

Edwards L J

Faba L

Faccin J E G

Ferguson N F

Ford E M

Frerichs K A

Frugé E D

Furness J B

Gannon N

Gerhart A J

Glencorse D

Goodband R D

Gourley K M

Grapentin J

Greenwood E C

Grupen C G

Guzmán-Pino S A

Hamilton D R

Hansen E L

Hansen S A

Harper J

Hayes M

Hazel S J

Hebert M

Heim G

Heller J

Hemsworth L M

Hemsworth P H

Henman D J

Heo J M

Hermesch S

Hernández-Jover M

Hetchler B

Hewitt R J E

Hiendleder S

Hieu H L
SunPork Group, Murarrie, QLD 4172, Australia

The University of Melbourne, Parkville, VIC 3010, Australia

Rivalea Australia Pty Ltd, Corowa, NSW 2646, Australia

SunPork Group, Murarrie, QLD 4172, Australia

Trouw Nutrition Innovation, 3811 MH Amersfoort, the Netherlands

Universidad de Chile, La Pintana, Santiago, Chile

Charles Sturt University, Wagga Wagga, NSW 2678, Australia

Ghent University, B-9000 Gent, Belgium

The University of Queensland, Gatton, QLD 4343, Australia

Novozymes A/S, 2880 Bagsværd, Denmark

Alltech, Nicholasville, KY 40356, Australia

Kansas State University, Manhattan, KS 66506, USA

Animal Welfare Science Centre, The University of Melbourne, Parkville, VIC 3010, Australia

Kansas State University, Manhattan, KS 66506, USA

Apiam Animal Health Limited, East Bendigo, VIC 3550, Australia

The University of Melbourne, Parkville, VIC 3010, Australia

University of Southern Queensland, Toowoomba, QLD 4350, Australia

ACE Livestock Consulting Pty Ltd, Cockatoo Valley, SA 5351, Australia

Animal Welfare Science Centre, The University of Melbourne, Parkville, VIC 3010, Australia

Ridley Agriproducts Pty Ltd, Pakenham, VIC 3810, Australia

Trouw Nutrition Innovation, 3811 MH Amersfoort, the Netherlands

Federal University of Rio Grande do Sul, Porto Alegre, RS 90040-060, Brazil

Trouw Nutrition R\&D, Burford, ON N0E 1A0, Canada

Rivalea Australia Pty Ltd, Corowa, NSW 2646, Australia

Hubbard Feeds, Mankato, MN 56001, USA

Hubbard Feeds, Mankato, MN 56001, USA

The University of Melbourne, Parkville, VIC 3010, Australia

Biomin Singapore Pte Ltd, Singapore 159471, Singapore

Hubbard Feeds, Mankato, MN 56001, USA

SunPork Group, Murarrie, QLD 4172, Australia

Kansas State University, Manhattan, KS 66506, USA

Kansas State University, Manhattan, KS 66506, USA

EW Nutrition GmbH, 49429 Visbek, Germany

The University of Adelaide, Roseworthy, SA 5371, Australia

The University of Sydney, Camden, NSW 2570, Australia

Universidad de Chile, La Pintana, Santiago, Chile

South Australian Research and Development Institute, Urrbrae, SA 5064, Australia

Hubbard Feeds, Mankato, MN 56001, USA

Hubbard Feeds, Mankato, MN 56001, USA

Rivalea Australia Pty Ltd, Corowa, NSW 2646, Australia

Animal Welfare Science Centre, The University of Melbourne, Parkville, VIC 3010, Australia

The University of Adelaide, Roseworthy, SA 5371, Australia

The University of Adelaide, Roseworthy, SA 5371, Australia

Trouw Nutrition Innovation, 3811 MH Amersfoort, the Netherlands

Charles Sturt University, Wagga Wagga, NSW 2678, Australia

Animal Welfare Science Centre, The University of Melbourne, Parkville, VIC 3010, Australia

Animal Welfare Science Centre, The University of Melbourne, Parkville, VIC 3010, Australia

Rivalea Australia Pty Ltd, Corowa, NSW 2646, Australia

Chungnam University, Daejeon 34134, Republic of Korea

Animal Genetics and Breeding Unit, University of New England, Armidale, NSW 2351, Australia

Charles Sturt University, Wagga Wagga, NSW 2678, Australia

University of Minnesota, St Paul, MN 55108, USA

SunPork Group, Murarrie, QLD 4172, Australia

The University of Adelaide, Roseworthy, SA 5371, Australia

The University of Melbourne, Parkville, VIC 3010, Australia 
Hilbrands A M

Hill I

Hine B C

Hong J S

Horgan K

Howard A J

$\mathrm{Hu} \mathrm{J}$

Hughes P E

Hutchings $S$

Janni K A

Jenkin S

Jenkins S N

Jing $\mathrm{H}$

Johnston L J

Johnston R

Jolley J Y C

Jordan D

Jung H I

Kelly J

Kerssen N

Kiermeier A

Kim I H

Kim J C

Kim Y B

Kim Y M

Kind $\mathrm{K}$

Kirkwood R N

Klapish E

Kocher A

Lahaye L

Laird T

Le H H

Lealiifano A K

Lester S

Leury B J

Li Y

Lines D S

Little S B

Liu D

Liu F

Lobo E T

Loudon E

Low W Y

Luo Y

Luo Y-H

Luxford B G

Macelline S P

Mallo J J

Mansfield J P

Maronich A

Marsh L

Mason M

Matsumoto N

McGahan E J

McKenny L A
University of Minnesota, Morris, MN 56267, USA

Bailey Creek Pork, Mulgildie, QLD 4630, Australia

CSIRO Agriculture and Food, Armidale, NSW 2350, Australia

Chungnam University, Daejeon 34134, Republic of Korea

Alltech Bioscience Centre, Dunboyne, Meath A86, Ireland

South Australian Research and Development Institute, Urrbrae, SA 5064, Australia

Dankook University, Cheonan 31116, Republic of Korea

Paul Hughes Consulting, North Adelaide, SA 5006, Australia

The University of Melbourne, Parkville, VIC 3010, Australia

University of Minnesota, St Paul, MN 55108, USA

Apiam Animal Health Limited, East Bendigo, VIC 3550, Australia

The University of Western Australia, Crawley, WA 6009, Australia

Dankook University, Cheonan 31116, Republic of Korea

University of Minnesota, Morris, MN 56267, USA

SunPork Group, Murarrie, QLD 4172, Australia

South Australian Research and Development Institute, Urrbrae, SA 5064, Australia

NSW Department of Primary Industries, Wollongbar, NSW 2477, Australia

Dankook University, Cheonan 31116, Republic of Korea

South Australian Research and Development Institute, Rosedale, SA 5350, Australia

Wageningen University and Research, 6708 PB Wageningen, the Netherlands

Statistical Process Improvement Consulting \& Training Pty Ltd, Gumeracha, SA 5233, Australia

Dankook University, Cheonan 31116, Republic of Korea

AB Vista, Marlborough, Wiltshire SN8 4AN, UK

Chungnam University, Daejeon 34134, Republic of Korea

Dankook University, Cheonan 31116, Republic of Korea

The University of Adelaide, Roseworthy, SA 5371, Australia

The University of Adelaide, Roseworthy, SA 5371, Australia

Rivalea Australia Pty Ltd, Corowa, NSW 2646, Australia

Alltech Lienert Australia Pty Ltd, Roseworthy, SA 5371, Australia

Jefo Nutrition Inc., Saint-Hyacinthe, QC J2R 2E7, Canada

Murdoch University, Murdoch, WA 6150, Australia

The University of Melbourne, Parkville, VIC 3010, Australia

Rivalea Australia Pty Ltd, Corowa, NSW 2646, Australia

APFoodIntegrity Pty Ltd, Grange, SA 5022, Australia

The University of Melbourne, Parkville, VIC 3010, Australia

University of Minnesota, Morris, MN 56267, USA

SunPork Group, Murarrie, QLD 4172, Australia

The University of Melbourne, Parkville, VIC 3010, Australia

KU Leuven, 3000 Leuven, Belgium

Rivalea Australia Pty Ltd, Corowa, NSW 2646, Australia

QAAFI, The University of Queensland, St Lucia, QLD 4067, Australia

Pork Innovation WA Inc., Viveash, WA 6056, Australia

The University of Adelaide, Roseworthy, SA 5371, Australia

DSM China Ltd, Shanghai 201203, People's Republic of China

Sichuan Agricultural University, Yaan, Sichuan 625014, People's Republic of China

Rivalea Australia Pty Ltd, Corowa, NSW 2646, Australia

Chungnam University, Daejeon 34134, Republic of Korea

NOREL S.A., Madrid 28007, Spain

Murdoch University, Murdoch, WA 6150, Australia

South Australian Research and Development Institute, Urrbrae, SA 5064, Australia

The University of Adelaide, Roseworthy, SA 5371, Australia

The University of Queensland, St Lucia, QLD 4067, Australia

Apiam Animal Health Limited, East Bendigo, VIC 3550, Australia

Integrity Ag \& Environment, Highfields, QLD 4352, Australia

South Australian Research and Development Institute, Roseworthy, SA 5371, Australia

viii 
McQuade R

Meaney D

Meers J

Mesas L

Meza K

Michiels J

Miller D W

Montalvo G

Monteiro A

Moore K L

Morales J

Morrison R S

Muller M

Musgrave I

Nahar N

Nairn K

Norton T

Nowland T L

O’Halloran K S

O'Dea M

Palmieri C

Parke C

Pedersen N R

Peelman L

Perez Calvo E

Phillips D

Plush K J

Pluske J M

Pluske J R

Pointon A M

Puyalto M

Rademacher-Heilshorn M

Rakhshandeh A

Rakhshandeh A R

Ralph C R

Ramos S H M

Rayner J

Reese M H

Ren W

Rice M

Rodríguez M

Rombola L

Romero L F

Roubos-van der Hil P

Roura E

Sahibzada S

Sampaio M O

Saunders D

Savage K

Schmeisser J

Segers L

Sharpe K T

Sherwood D

Shi $\mathrm{H}$

Siegford J
The University of Melbourne, Parkville, VIC 3010, Australia

Biomin Singapore Pte Ltd, Singapore 159471, Singapore

The University of Queensland, Gatton, QLD 4343, Australia

NOREL S.A., Madrid 28007, Spain

Montana S.A., La Molina, Lima 12, Peru

Ghent University, B-9000 Gent, Belgium

Murdoch University, Murdoch, WA 6150, Australia

PigCHAMP Pro Europa, 40006 Segovia, Spain

ANIMINE, 74960 Meythet, Annecy, France

Pork Innovation WA Inc., Viveash, WA 6056, Australia

PigCHAMP Pro Europa, 40006 Segovia, Spain

Rivalea Australia Pty Ltd, Corowa, NSW 2646, Australia

QAAFI, The University of Queensland, St Lucia, QLD 4067, Australia

The University of Adelaide, Adelaide, SA 5000, Australia

QAAFI, The University of Queensland, St Lucia, QLD 4067, Australia

Peel Pork, Yarloop, WA 6218, Australia

KU Leuven, 3000 Leuven, Belgium

The University of Adelaide, Roseworthy, SA 5371, Australia

Rivalea Australia Pty Ltd, Corowa, NSW 2646, Australia

Murdoch University, Murdoch, WA 6150, Australia

The University of Queensland, Gatton, QLD 4343, Australia

The University of Queensland, Gatton, QLD 4343, Australia

Novozymes A/S, 2880 Bagsværd, Denmark

Ghent University, B-9000 Gent, Belgium

DSM Nutritional Products France, 68128 Village-Neuf, France

The University of Melbourne, Parkville, VIC 3010, Australia

SunPork Group, Murarrie, QLD 4172, Australia

SciEcons Consulting, Subiaco, WA 6904, Australia

Murdoch University, Murdoch, WA 6150, Australia

APFoodIntegrity Pty Ltd, Grange, SA 5022, Australia

NOREL S.A., Madrid 28007, Spain

Evonik Nutrition and Care GmbH, 45128 Essen, Germany

Texas Tech University, Lubbock, TX 79409, USA

South Plains College, Levelland, TX 79336, USA

Agersens Pty Ltd, Camberwell, VIC 3124, Australia

Evonik (SEA) Pte Ltd, Singapore 609927, Singapore

South Australian Research and Development Institute, Roseworthy, SA 5371, Australia

University of Minnesota, Morris, MN 56267, USA

Sichuan Agricultural University, Yaan, Sichuan 625014, People's Republic of China

Animal Welfare Science Centre, The University of Melbourne, Parkville, VIC 3010, Australia

PigCHAMP Pro Europa, 40006 Segovia, Spain

Orffa Additives BV, 4251 LC Werkendam, the Netherlands

DSM Nutritional Products Switzerland, 4503 Kaiseraugst, Switzerland

Trouw Nutrition Innovation, 3811 MH Amersfoort, the Netherlands

QAAFI, The University of Queensland, St Lucia, QLD 4067, Australia

Murdoch University, Murdoch, WA 6150, Australia

SunPork Group, Murarrie, QLD 4172, Australia

Link Asia Partners Pte Ltd, 408568 Singapore

Apiam Animal Health Limited, East Bendigo, VIC 3550, Australia

DSM Nutritional Products France, 68128 Village-Neuf, France

Orffa Additives BV, 4251 LC Werkendam, the Netherlands

University of Minnesota, Morris, MN 56267, USA

EW Nutrition GmbH, 49429 Visbek, Germany

Dankook University, Cheonan 31116, Republic of Korea

Michigan State University, East Lansing, MI 48824, USA 
Sinclair M

Smith P

Smith S

Smits R J

Smyth D

Sol C

Stamatopoulos K

Staveley L M

Steel A N

Steibel J

Stein $\mathrm{H} \mathrm{H}$

Stensland I

Sterndale S O

Sumner J

Swanson A J

Tactacan G

Tallaksen J E

Taylor-Pickard J A

Terry R

Tilbrook A

Tokach M D

Torok V A

Trezona M

Tritton S M

Turni C

Turpin D L

Upadhaya S D

van Barneveld R J

van Dissel J

Van Liefferinge E

Van Noten M

Van Poucke M

van Sliedregt $\mathrm{H}$

van Wettere W H E J

Vargovic L

Verlhac Trichet V

Vidacs E

Waite I S

Walker J

Walsh M C

Wang $S$

Ward S A

Watson K J L

Watt A

Wheat L

Whittaker A

Wickramasuriya S S

Wiedemann S G

Wijesiriwardana D A

Wilkinson A R

Woodworth J C

Wooten $\mathrm{H}$

$\mathrm{Wu} \mathrm{J}$

Yee S

Yu B
Orffa Additives BV, 4251 LC Werkendam, the Netherlands

Australian Pork Limited, Barton, ACT 2600, Australia

Rivalea Australia Pty Ltd, Corowa, NSW 2646, Australia

Rivalea Australia Pty Ltd, Corowa, NSW 2646, Australia

South Australian Research and Development Institute, Urrbrae, SA 5064, Australia

NOREL S.A., Madrid 28007, Spain

DSM Nutritional Products Australia Pty Ltd, Wagga Wagga, NSW 2650, Australia

SunPork Group, Murarrie, QLD 4172, Australia

The University of Sydney, Camden, NSW 2570, Australia

Michigan State University, East Lansing, MI 48824, USA

University of Illinois at Urbana-Champaign, Champaign 61801, USA

Linley Valley Pork, Wundowie, WA 6560, Australia

Murdoch University, Murdoch, WA 6150, Australia

M \& S Food Consultants Pty Ltd, Deviot, TAS 7275, Australia

Kansas State University, Manhattan, KS 66506, USA

Jefo Nutrition Inc., Saint-Hyacinthe, QC J2R 2E7, Canada

University of Minnesota, Morris, MN 56267, USA

Alltech Bioscience Centre, Dunboyne, Meath A86, Ireland

South Australian Research and Development Institute, Roseworthy, SA 5371, Australia

QAAFI, The University of Queensland, St Lucia, QLD 4067, Australia

Kansas State University, Manhattan, KS 66506, USA

South Australian Research and Development Institute, Adelaide, SA 5000, Australia

Linley Valley Pork, Wundowie, WA 6560, Australia

SunPork Group, Murarrie, QLD 4172, Australia

QAAFI, The University of Queensland, St Lucia, QLD 4067, Australia

Murdoch University, Murdoch, WA 6150, Australia

Dankook University, Cheonan 31116, Republic of Korea

SunPork Group, Murarrie, QLD 4172, Australia

The University of Adelaide, Roseworthy, SA 5371, Australia

Ghent University, B-9000 Gent, Belgium

Ghent University, B-9000 Gent, Belgium

Ghent University, B-9000 Gent, Belgium

Riverbend Pork Group, Toowoomba, QLD 4350, Australia

The University of Adelaide, Roseworthy, SA 5371, Australia

Animal Genetics and Breeding Unit, University of New England, Armidale, NSW 2351, Australia

DSM Nutritional Products Switzerland, 4503 Kaiseraugst, Switzerland

The University of Melbourne, Parkville, VIC 3010, Australia

The University of Western Australia, Crawley, WA 6009, Australia

Rivalea Australia Pty Ltd, Corowa, NSW 2646, Australia

DSM Nutritional Products Switzerland, 4503 Kaiseraugst, Switzerland

DSM China Ltd, Shanghai 201203, People's Republic of China

The University of Adelaide, Roseworthy, SA 5371, Australia

Integrity Ag \& Environment, Highfields, QLD 4352, Australia

The University of Melbourne, Parkville, VIC 3010, Australia

Future Green Solutions Pty Ltd, Geraldton, WA 6530, Australia

The University of Adelaide, Roseworthy, SA 5371, Australia

Chungnam University, Daejeon 34134, Republic of Korea

Integrity Ag \& Environment, Highfields, QLD 4352, Australia

The University of Melbourne, Parkville, VIC 3010, Australia

Rivalea Australia Pty Ltd, Corowa, NSW 2646, Australia

Kansas State University, Manhattan, KS 66506, USA

Texas Tech University, Lubbock, TX 79409, USA

DSM China Ltd, Shanghai 201203, People's Republic of China

QAAFI, The University of Queensland, St Lucia, QLD 4067, Australia

Sichuan Agricultural University, Yaan, Sichuan 625014, People's Republic of China 
Zentek J

Zhai H-X

Zhang S

Zhao W

Zhu W
Freie Universität Berlin, 14195 Berlin, Germany

DSM China Ltd, Shanghai 201203, People's Republic of China

Dankook University, Cheonan 31116, Republic of Korea

The University of Melbourne, Parkville, VIC 3010, Australia

Jiangsu University, Zhenjiang 212013, Jiangsu, People's Republic of China 


\section{Acknowledgements to referees}

The proceedings of the seventeenth biennial conference of the Australasian Pig Science Association, 'Manipulating Pig Production XVII', contains 99 one-page papers, 6 review papers, 3 symposia papers and the paper presented as the Dunkin Memorial Lecture. As is the policy of the Association, all papers were reviewed by external referees. The APSA Committee would like to extend our sincerest thanks to Dr John Patience for his role as Guest Editor for Animal. The APSA Committee and Editors gratefully acknowledge the assistance generously given during 2019 by the following referees:

$\begin{array}{llll}\text { Abraham S } & \text { Doyle RE } & \text { Kim JC } & \text { Richards KJ } \\ \text { Athorn RZ } & \text { D'Souza DN } & \text { King RH } & \text { Rikard-Bell CV } \\ \text { Ball RO } & \text { Edwards AC } & \text { Kirkwood RN } & \text { Roura E } \\ \text { Black JL } & \text { Edwards MV } & \text { Lealiifano AK } & \text { Smits RJ } \\ \text { Brewster CJ } & \text { Gannon NJ } & \text { Lines DS } & \text { Stein HH } \\ \text { Bunter KL } & \text { Gleeson BL } & \text { Luxford BG } & \text { Terry R } \\ \text { Cadogan DJ } & \text { Grieve A } & \text { Mitchell PM } & \text { Tilbrook AJ } \\ \text { Campbell RG } & \text { Grupen CG } & \text { Moore KL } & \text { Tokach MD } \\ \text { Channon HA } & \text { Hemsworth LM } & \text { Morrison RS } & \text { Trezona M } \\ \text { Collins CL } & \text { Hemsworth PH } & \text { Mullan BP } & \text { Tritton SM } \\ \text { Condous PC } & \text { Henman DJ } & \text { Muller TL } & \text { Turni C } \\ \text { Cottrell JJ } & \text { Hermesch S } & \text { Plush KJ } & \text { van Barneveld RJ } \\ \text { Cutler RS } & \text { Hewitt RJE } & \text { Pluske JR } & \text { van Breda LK } \\ \text { DeRouchey JM } & \text { Holyoake PK } & \text { Pointon AM } & \text { van Sliedregt H } \\ \text { DiGiacomo K } & \text { Jolley JYC } & \text { Pollock YJ } & \text { van Wettere WHEJ } \\ \text { Downing JA } & \text { Jongman EC } & \text { Ralph CR } & \text { Wilkinson S }\end{array}$




\section{Summary List}

s01 Evaluation of changes in plasma biomarkers of gastrointestinal functionality in early weaned piglets in response to high protein diets and feed restriction

E Perez Calvo, E Bacou, J Schmeisser, V Verlhac Trichet, L F Romero, M C Walsh and P Celi

s02 Satiating responses to branched chain amino acids are mediated through ileal GLP-1 secretion in postweaning pigs

M Muller, A Tilbrook, R J van Barneveld and E Roura

s03 Gene expression of Transient Receptor Potential Ankyrin 1 and Transient Receptor Potential Vanilloid 1 in the gastro-intestinal tract of piglets during the peri-weaning period

E Van Liefferinge, N Van Noten, J Degroote, M Van Poucke, L Peelman, E Roura and J Michiels

s04 The effect of added phytase in weaner pig diets on intestinal alkaline phosphatase activity in the mucosa of the small intestine

I Stensland, J C Kim, J P Mansfield, D L Turpin, M R Bedford and J R Pluske

s05 Supplementation of xylo-oligosaccharides plus xylanase increased VFA and decreased BCFA production in weaned pigs

H M Cho, S S Wickramasuriya, S P Macelline, J S Hong, Y B Kim, J C Kim, G Cordero, M R Bedford and J $\mathrm{M} \mathrm{Heo}$

s06 Effects of glucocorticoid receptor agonist on apparent ileal and total tract digestibility of dietary nutrients and the histomorphology of the small intestine in newly weaned pigs

H Wooten, A R Rakhshandeh and A Rakhshandeh

s07 Effect of glucocorticoid administered to sows at farrowing on piglet performance

S A Ward, R N Kirkwood, D S Lines, K J Plush

S08 Non-linear associations were found between litter size and sow survival to the next parity

$\mathrm{N}$ Kerssen and S Hermesch

s09 Differences in farrowing rate according to parity and trait specific temperature groupings around mating events

A M G Bunz, K L Bunter, R S Morrison, B G Luxford and S Hermesch

s10 A comparison of different physiological temperature measurements of lactating pure and crossbred lines sows over summer

S L Beer, F Liu and S Smith

s11 Heat stress during mid-gestation up-regulates placental insulin-like growth factor 2 (IGF-2) mRNA

expression and affects hormonal profiles in pregnant gilts

W Zhao, F Liu, A W Bell, J J Cottrell, B J Leury and F R Dunshea

s12 A comparison of placenta and piglet birth weight of sows mated in summer and autumn

F Liu, E M Ford, R J Smits, R S Morrison, C J Brewster, D J Henman, W Zhao, B J Leury, J J Cottrell, F R

Dunshea and A W Bell

s13 Birth weight is an early indicator of reproductive development in the gilt

L M Staveley, K Kind, J Kelly and W H E J van Wettere

s14 What factors recorded before farrowing affect sow's ability to wean piglets?

L Vargovic, K L Bunter, S Hermesch, R S Morrison and R Johnston

s15 Increasing essential amino acids in late gestation improves birth weight in multiparous sows

D J Cadogan, I Hill, H van Sliedregt and R O Ball

s16 Evaluation of sow and piglet characteristics related to stillborn rate and pre-weaning survival in high producing sows

K M Gourley, H I Calderon, A J Swanson, J C Woodworth, J M DeRouchey, M D Tokach, R D Goodband and S S Dritz 
s17 Anti-inflammatory administration to sows prior to farrowing does not improve piglet survival or growth K J Plush, J R Pluske, D S Lines, C R Ralph and R N Kirkwood

s18 Dextrose supplementation in the wean to mate period increases litter size and maintains piglet viability D Glencorse, J G Alexopoulos, S M Tritton, D N D’Souza and K J Plush

s19 Dietary 1,3-Butanediol increases weight and decreases small for age progeny at birth D A Wijesiriwardana, J R Craig, J R Pluske, J J Cottrell and F R Dunshea

s20 Supplementing guanidinoacetic acid in the gestating and lactating sow diet improves lactation feed intake and body condition of sows at weaning

J R Craig, K S O’Halloran, S L Beer, F Liu, M Rademacher-Heilshorn, S H M Ramos and D J Henman

s21 Black soldier fly technology can convert piggery manure into valuable fertiliser

S N Jenkins, I S Waite, L K Abbott and L Wheat

s22 Quantifying use of electricity to heat piglet creep areas in farrowing rooms

L J Johnston, K T Sharpe, M H Reese, E S Buchanan, J E Tallaksen, A M Hilbrands, K A Janni, B Hetchler and $\mathrm{Y} \mathrm{Li}$

s23 Trends in the environmental impacts from the Australian pork industry: 1980-2020

K J L Watson, S G Wiedemann, L Biggs and E J McGahan

s24 Effects of weaning age and antibiotic use on pig performance in a commercial system

J E G Faccin, M W Allerson, S S Dritz, M D Tokach, J C Woodworth, R D Goodband, J M DeRouchey and F

P Bortolozzo

s25 The effect of Lactobacillus and yeast ferment-based additives on post-weaning diarrhoea in enterotoxigenic Escherichia coli challenged piglets

T Laird, J P Mansfield, S Sahibzada, D L Turpin, J R Pluske, S Abraham and M O’Dea

s26 A multiplex molecular assay for Glaesserella australis

S Yee, N Nahar, E T Lobo, S Jenkin and C Turni

s27 The association between sire immune competence and antibody response of progeny

J Harper, K L Bunter, S Hermesch and B C Hine

s28 Faecal microbiota transplantation during lactation had no effect on piglets to weaning

T L Nowland, W Y Low, V A Torok, M D Barton, K J Plush and R N Kirkwood

s29 Rapid and easy extraction method using cellulose dipstick in oral fluid samples

A R dela Cruz, J Meers, T S Barnes, C Palmieri, C Parke, M Mason, P J Blackall, J Botella and C Turni

s30 Prevalence of MRSA in Australian pig herds and molecular typing of isolates

S Sahibzada, M Hernández-Jover, D Jordan, S Abraham, M O'Dea and J Heller

s31 Coarse milled wheat inclusion in a pelleted feed provides no health benefits and reduces feed efficiency in growing, but not finishing pigs

C J Brewster, E Klapish and D J Henman

s32 Effect of standardized ileal digestible lysine and added copper on growth performance, nutrient digestibility, and backfat thickness in growing pigs

Y M Kim, X Ao, J Hu, S Zhang and I H Kim

s33 Feeding Lupinus albus L. to immunocastrated male pigs to reduce feed intake and backfat K L Moore, E Loudon and F R Dunshea

s34 Disappearance of dietary fiber in the small intestine, large intestine, and total tract of growing pigs fed cornor wheat-based diets without or with microbial xylanase

$\mathrm{J} \mathrm{J}$ Abelilla and $\mathrm{H}$ H Stein

s35 Isoquinoline alkaloids and betaine supplementation ameliorate heat stress in grower pigs

H L Hieu, D Phillips, E Vidacs, W Zhao, J B Furness, R McQuade, F R Dunshea, V Artuso-Ponte and J J Cottrell 
s36 Rapid eating-quality assessment of premium Australian pork in an Asian market E P Bittner, R J van Barneveld, D N D’Souza, H Ashman, S Hutchings and F R Dunshea

s37 Pork sensory quality fail rates can be due to pigs from different supply chains varying in breed and rearing environments and fed novel diet ingredients

R J Smits, A K Lealiifano, H A Channon, J Y C Jolley, D Smyth, A Maronich and P Smith

s38 Colour difference between free range and conventional pork from entire males is greatest during spring M Trezona, K L Moore, I Stensland

s39 Review of pig post-mortem inspection and disposition schedules of Australian Standard 4696 A M Pointon, D R Hamilton, A Kiermeier

s40 An improved microbiological monitoring system for the Australian pork industry - an industry trial J Y C Jolley, A Kiermeier and J Sumner

s41 Exploratory behaviour and general locomotor activity of Sprague Dawley rats (Rattus norvegicus) following repeated daily exposure to several anticoagulant rodenticides (AR)

A J Howard, D R Hamilton, I Musgrave and J Y C Jolley

s42 Provision of straw or cotton rope to sows may result in more piglets being born alive R S Morrison, L E Edwards, S L Beer, K J Plush and R E Doyle

s43 Positive human contact reduces avoidance behaviour of gestating sows during routine husbandry procedures M Hayes, L M Hemsworth, R S Morrison, M Rice and P H Hemsworth

s44 Corticosteroid administration can mitigate the stress response of post-weaned pigs exposed to mixing S O Sterndale, D W Miller, J P Mansfield, J C Kim and J R Pluske

s45 Minimal increases in space allowance during the nursery phase in a commercial setting improves performance and health

D L Turpin and J R Pluske

s46 The analgesic effect of three pain relief protocols on behaviour in castrated piglets N Matsumoto, D L Turpin, A Ballares, R H Dunlop, G Chuck, K Savage and K Nairn

s47 Validation of routine visual only post-mortem inspection of pigs in Australia A M Pointon, D R Hamilton and A Kiermeier

s48 Pig risk-based review of post-mortem inspection of kidneys of pigs in Australia A M Pointon, D R Hamilton and A Kiermeier

s49 Antibiotic use and the expression of wild type patterns for Enterococcus species isolates: A comparison of three different pork production systems

D L Turpin, J M Pluske and J R Pluske

s50 Impact of water quality on the solubility of veterinary antibiotics administered to pigs through drinking water L J Edwards, S B Little, A Watt and H Billman-Jacobe

s51 Competitive exclusion by promoting growth of beneficial bacteria after Salmonella infection $\mathrm{N}$ de Groot, L Faba and P Roubos-van der Hil

s52 Organic acids in drinking water improve water intake and performance of nursery piglets - a meta-analysis $\mathrm{N}$ de Groot, G Heim and N F Ferguson

s53 Impact of dietary fibre type and content on Escherichia coli shedding and microbial structure after weaning S N Jenkins, I S Waite, J P Mansfield, J C Kim and J R Pluske

s54 Xylo-oligosaccharide and xylanase improved growth performance in weaner pigs fed an antibiotic free low $\mathrm{ZnO}$ diet when exposed to an unsanitary environment H M Cho, S S Wickramasuriya, S P Macelline, J S Hong, Y B Kim, J C Kim, G Cordero, M R Bedford and J $\mathrm{MHeo}$ 
s55 Feeding larger diameter pellets to piglets in lactation does not improve the weaning weight of piglets born to gilts but decreases the removal rate of pigs in the post-weaning period

J R Pluske, J C Kim, J R Craig, R J Smits, C J Braden and C J Brewster

s56 Phytogenic feed additives and dietary energy density can be used to improve the growth performance of pigs between 20 and $95 \mathrm{~kg}$ live weight.

J Walker, D J Henman, S L Beer, D Meaney and N Gannon

s57 Improving growth performance of 6 to $13 \mathrm{~kg}$ nursery pigs

K Horgan, N Browne, J A Taylor-Pickard, E D Frugé, A J Gerhart, E L Hansen, S A Hansen and K A Frerichs

s58 Lupin cell-wall in vitro solubilisation with combination of exogenous feed enzyme products K Stamatopoulos, E A Della Pia and N R Pedersen

s59 Evaluation of different feed acidification strategies for improvement of growth performance and economics of nursery pigs under commercial conditions

L Lahaye, G Tactacan, and R G Campbell

s60 Soybean meal in weaner diets improves growth performance, when compared with other soybean products M O Sampaio, R J E Hewitt, S M Tritton, A C Corso, D N D’Souza and R J van Barneveld

s61 Including grape marc in weaner diets reduces growth performance when fed at higher levels M O Sampaio, R J E Hewitt, S M Tritton, A C Corso, D N D'Souza and R J van Barneveld

s62 Effect of organic trace minerals in finisher pig diets on growth performance and loin muscle depth at slaughter

A R Wilkinson, D J Henman, C J Brewster, A K Lealiifano, A Kocher and R M Delles

s63 Influence of Bacillus-based probiotic complex on growth performance, nutrient digestibility, blood metabolites, and faecal E.coli and Lactobacillus counts in weaner pigs

S D Upadhaya, H Jing, H I Jung and I H Kim

s64 Increased glutamate intake did not result in higher levels in milk when supplemented to gestating and lactating sow diets

V De Luca, E Roura and S A Guzmán-Pino

s65 Glutamate inclusion in gestating and lactating diets in sows increased sweet taste sensitivity and appetite in postweaning pigs

V De Luca, E Roura and S A Guzmán-Pino

s66 Defining temperature groupings around mating of sows for farrowing rate using cluster analysis A M G Bunz, K L Bunter, R S Morrison, B G Luxford and S Hermesch

s67 Relationship between serum anti-Müllerian hormone levels and ovarian reserve in gilts A N Steel, R Z Athorn and C G Grupen

s68 Fence-line boar exposure at the end of lactation did not improve the reproductive performance of group housed sows

E M Ford, E C Greenwood, R Z Athorn, R S Morrison and W H E J van Wettere

s69 Piglet birth weight does not correlate with umbilical venous-arterial glucose difference measured at birth F Liu, B J Leury, E M Ford, R J Smits, R S Morrison, C J Brewster, D J Henman, W Zhao, J J Cottrell, F R Dunshea and A W Bell

s70 The impact of assistance at farrowing on subsequent reproduction of sows J G Alexopoulos and K J Plush

s71 The effect of fostering status and nurse sow parity on pre-weaning survival J Harper and K L Bunter

s72 Can we predict shortened or prolonged gestation upon transfer to the farrowing shed? L Vargovic, K L Bunter, S Hermesch, P C Condous, J Harper 
s73 Thermal imaging of skin but not eye can be used as an alternative body temperature measurement in lactating sows in a hot environment.

S L Beer, A M G Bunz, J Harper, F Liu and S Smith

s74 Risk-based assessment of criteria used for disposition judgement for polyarthritis of pigs A M Pointon, D R Hamilton, S Lester and A Kiermeier

s75 Validation of alternative post-mortem inspection procedures with reference to pleurisy of pigs in Australia A M Pointon, D R Hamilton and A Kiermeier

s76 Dietary supplementation of a Bacillus licheniformis derived-protease, alone or in combination with valine to a lower protein diet improves growth performance in pigs

J Hu, L Cheng and I H Kim

s77 Effects of low dose $\mathrm{ZnO}$ combined with probiotic complex relative to high dose $\mathrm{ZnO}$ on the performance, nutrient digestibility and faecal microbial counts in weaner pigs

S D Upadhaya, H Shi, H Jing and I H Kim

s78 Dexamethasone administrations can reduce intestinal permeability in weaner pigs exposed to mixing stress S O Sterndale, D W Miller, J P Mansfield, J C Kim and J R Pluske

s79 Effect of imprinting with essential oils on performance and health parameters in weaned piglets B Canal, M Puyalto, L Mesas, C Sol, R Chaves, V Cantarelli and J J Mallo

s80 Efficacy of medium chain fatty acid based additive at graded dose levels on performance and faecal consistency in piglets

C Sol, M Puyalto, J J Mallo and J Zentek

s81 Effects of dietary supplementation of non-starch polysaccharide enzymes on the growth performance and nutrient digestibility of grower-finisher pigs

Y Luo, H Zhai, S Wang and J Wu

s82 Dietary caffeine reduces feed intake and improves feed efficiency in female grower pigs

C J Braden, R S Morrison, F Liu and C J Brewster

s83 Feeding the same diet to pigs in the grower-finisher period reduces feed costs compared to phase feeding with no effect on growth performance and carcass quality

K L Moore, T Clarke and A C Edwards

s84 Effect of a nutritional emulsifier in an energy reduced diet on the performance of fattening pigs

K Meza, L Rombola, M Sinclair, B Bruneel and L Segers

s85 Is it possible to reduce zinc supplementation in nursery piglets without affecting growth performance? M Rodríguez, A Monteiro, G Montalvo, I Cockshott, D Saunders and J Morales

s86 The inclusion of phytomolecules in weaner pig diets improves growth performance

R J E Hewitt, F F Barbosa, J Grapentin, D Sherwood, S M Tritton, A C Corso and D N D'Souza

s87 Effects of Prevotella/Bacteroides ratio and dietary starch type on SCFAs concentrations, composition of butyrate-producing bacteria and gene expression in finishing pigs

W Ren, Y-H Luo, B Yu, D-W Chen, H Zhai and M C Walsh

s88 Use of novel photoperiod schedules for stimulating weaner performance

J R Craig, R S Morrison, D J Henman, C J Brewster, E M de Ruyter and K S O’Halloran

s89 Sorting newly weaned pigs by body weight in a commercial setting takes more time and does not improve performance

D L Turpin and J R Pluske

s90 Farm differences, but not novel dietary ingredients, affects carcass and meat quality of pork R J Smits, A K Lealiifano, R Aitken, H A Channon and P Smith

s91 Recognition of high-speed aggressive interactions of pigs on group level by depth sensor

C Chen, W Zhu, D Liu, J Steibel, J Siegford, T Norton

xvii 
s92 Mixing sows into group lactation housing shows marked differences between multisuckle and sow separation systems

E C Greenwood, J van Dissel, J Rayner, P E Hughes and W H E J van Wettere

s93 Pronounced inter-individual variation in plasma cortisol response to fluoxetine hydrochloride in the pig L Marsh, S Hiendleder1, A Whittaker, R Terry and C R Ralph

s94 Hessian stimulates nest building behaviour in sows and the provision of straw improves traits important for piglet survival

L A McKenny, K J Plush, T L Nowland and W H E J van Wettere

s95 The provision of straw within a farrowing crate improves piglet survival

L A McKenny, K J Plush, T L Nowland and W H E J van Wettere

s96 Systematic review and meta-analysis of pre-weaning mortality for farrowing crates and pens

D Glencorse, K J Plush, S J Hazel, D N D'Souza and M Hebert

s97 Use of a synthetic olfactory agonist in the farrowing house

R Terry, T L Nowland and K J Plush

s98 Preliminary study: A novel snout cooling system in farrowing huts reduced stillbirths in free range sows during summer

M Trezona, I Stensland, E Loudon, Z Cochran and S Dutton

s99 Proof of concept: Does snout cooling in farrowing huts during summer encourage free range sows to remain with piglets for longer periods during early lactation?

M Trezona, I Stensland, E Loudon, Z Cochran and S Dutton 


\title{
Evaluation of changes in plasma biomarkers of gastrointestinal functionality in early weaned piglets in response to high protein diets and feed restriction
}

\author{
$\underline{\text { E Perez Calvo }}^{1}$, E Bacou ${ }^{1}$, J Schmeisser ${ }^{1}$, V Verlhac Trichet ${ }^{2}$, L F Romero ${ }^{2}, \mathrm{M} \mathrm{C} \mathrm{Walsh}^{2}$ and P Celi ${ }^{3}$ \\ ${ }^{1}$ DSM Nutritional Products, France, ${ }^{2}$ DSM Nutritional Products, Switzerland, ${ }^{3}$ DSM Nutritional Products, USA \\ estefania.perez-calvo@dsm.com
}

\begin{abstract}
Application Understanding of the impact of high protein diet and feed restriction in plasma biomarkers of gastrointestinal functionality in early weaned to use it as a model to evaluate different nutritional strategies
\end{abstract}

Introduction The process of weaning imposes simultaneous stressors which compromise gastrointestinal functionality, including impairment of intestinal integrity, disturbance of digestive and absorptive capacity, alteration of the microbiota and immune system and increases in oxidative stress. These stressors result in clinical signs like post-weaning diarrhoea and reduced growth and feed intake, particularly during the first week after weaning. Both leguminous plant proteins and high level of dietary proteins can influence gastrointestinal functionality in weaned piglets (Pluske et al., 2002). Feed deprivation impacts nutrient absorption, gut secretions, and growth performance (Lalles et al., 2004). The hypothesis of this study was that early weaned piglets fed a high protein diet containing soybean meal plus feed restriction events 4-5 and 11-12 days post-weaning would result in changes in plasma biomarkers of gastrointestinal functionality.

Materials and methods A total of 32 male pigs weaned at 21 days with initial BW of $5.7 \pm 0.9 \mathrm{~kg}$ were used in a 2 week trial. There were two treatments that consisted of 1) low protein diet (LP; $182 \mathrm{~g} \mathrm{of} \mathrm{CP} / \mathrm{kg}$ ) offered ad libitum and 2) high protein diet (246 g of CP/kg) including feed restriction between 0800 and $1400 \mathrm{~h}$ during days 4-5 and 11-12 of the study (HP-R). Faecal scores were assessed daily using a scoring system from 0 to 3 . Individual piglet weights and blood samples were taken on d 0,6 and 13. Plasma urea nitrogen (PUN) and alkaline phosphatase activity (APA) were determined using Cobas 6000. Vitamin E (alpha-tocopherol) and Vitamin A (retinol) of heparin plasma concentrations were assayed using liquid chromatography (HPLC). Superoxide dismutase (SOD) and catalase (CAT) activities were analysed using commercial colorimetric assay kits. The concentration of hydroperoxides (d-ROMs test) and plasma antioxidant potential (PAT test) were assayed using commercial kits on a FRAS5 instrument (H\&D, Parma, Italy), and the oxidative stress index (OSI) was calculated as the ratio of d-ROMs to PAT. Data were analysed as a 2 × 2 factorial with piglet as the experimental unit. The model included time to weaning, diet, and time $\mathrm{x}$ diet as fixed effects and replicate as random. $\chi^{2}$ test was used to analyse frequency of diarrhoea between treatments. Statistical significance was considered at $P<0.05$.

Results No significant differences in average daily weight gain ( $69 \pm 30.6$ vs $98 \pm 59.4 \mathrm{~g} / \mathrm{d})$ between the LP ad libitum and HP-R fed piglets were observed during the two weeks. Feeding HP-R diets increased diarrhoea frequency $(P=0.04)$ considering diarrhoea as faecal score 2 and 3. PUN concentration increased, while SOD activity decreased when piglets were fed with an HP-R diet, especially 6 days after weaning (Table 1). In addition, the OSI tended to be higher after weaning in piglets fed with an HP-R diet. Overall, plasma concentration of vitamin A and E, and CAT and APA activities decreased over time. Redox balance was also affected by time in that d- ROMs and OSI values increased over time, while PAT levels decreased. The increase in OSI tended to be higher in the HP-R fed piglets $(P<0.09)$.

Table 1. Plasma biomarkers of gastrointestinal functionality from d 0 to $\mathrm{d} 13$ post-weaning according to diet treatment.

\begin{tabular}{|c|c|c|c|c|c|c|c|c|c|}
\hline \multirow{2}{*}{$\begin{array}{l}\text { Time to weaning }(\mathrm{d}) \\
\text { Parameters }\end{array}$} & \multirow[t]{2}{*}{ d 0} & \multicolumn{2}{|c|}{ d 6} & \multicolumn{2}{|c|}{ d 13} & \multirow[b]{2}{*}{ SEM } & \multicolumn{3}{|c|}{$P$ value } \\
\hline & & LP & HP-R & LP & HP-R & & Time $(\mathrm{T})$ & $\operatorname{Diet}(\mathrm{D})$ & $\mathrm{T}^{*} \mathrm{D}$ \\
\hline PUN (mg/dl) & 17.64 & 15.04 & 28.27 & 14.83 & 20.29 & 1.38 & 0.29 & 0.006 & 0.24 \\
\hline $\mathrm{APA}(\mathrm{U} / \mathrm{L})$ & $525.0^{\mathrm{a}}$ & $235.3^{\mathrm{b}}$ & $201.4^{\mathrm{b}}$ & $269.6^{\mathrm{ab}}$ & $269.2^{\mathrm{b}}$ & 21.6 & $<0.001$ & 0.75 & 0.67 \\
\hline Vitamin A (ng/mg) & $161.0^{\mathrm{b}}$ & $173.9^{\mathrm{b}}$ & $177.4^{\mathrm{ab}}$ & $259.0^{\mathrm{a}}$ & $258.0^{\mathrm{a}}$ & 9.6 & $<0.001$ & 0.49 & 0.72 \\
\hline Vitamin E (ng/mg) & $4606^{\mathrm{a}}$ & $1879^{b}$ & $2511^{\mathrm{b}}$ & $1450^{b}$ & $1693^{\mathrm{ab}}$ & 233 & $<0.001$ & 0.20 & 0.82 \\
\hline $\mathrm{SOD}(\mathrm{U} / \mathrm{mL})$ & 5.68 & 5.33 & 4.26 & 6.89 & 7.08 & 0.35 & 0.26 & 0.024 & 0.59 \\
\hline CAT (U/mL) & $899.1^{\mathrm{ab}}$ & $580.4^{\mathrm{b}}$ & $589.4^{\mathrm{b}}$ & $416.5^{\mathrm{bc}}$ & $559.4^{\mathrm{b}}$ & 37.3 & $<0.001$ & 0.64 & 0.02 \\
\hline d-ROMs (U.Carr*) & $688^{\mathrm{b}}$ & $920^{\mathrm{a}}$ & $917^{\mathrm{ab}}$ & $825^{\mathrm{ab}}$ & $940^{\mathrm{a}}$ & 30.4 & 0.009 & 0.40 & 0.65 \\
\hline PAT (U.Cor**) & $2809^{a}$ & $2547^{\mathrm{ab}}$ & $2033^{b}$ & $2349^{b}$ & $2395^{\mathrm{ab}}$ & 478 & 0.012 & 0.20 & 0.16 \\
\hline OSI (arbitrary units) & $0.24^{\mathrm{b}}$ & $0.37^{\mathrm{ab}}$ & $0.54^{\mathrm{a}}$ & $0.35^{\mathrm{ab}}$ & $0.41^{\mathrm{a}}$ & 0.02 & 0.005 & 0.09 & 0.35 \\
\hline
\end{tabular}

${ }_{\mathrm{a}, \mathrm{b}, \mathrm{c} M e a n s}$ within a row with different superscripts differ significantly $(P<0.05)$; LP= low protein $\operatorname{diet}(182 \mathrm{~g} \mathrm{of} \mathrm{CP} / \mathrm{kg})$ offered ad libitum; HP-R= high protein diet $(246 \mathrm{~g}$ of $\mathrm{CP} / \mathrm{kg})$ including feed restriction between 0800 and $1400 \mathrm{~h}$ during days 4-5 and 11-12 of the study; PUN= Plasma Urea Nitrogen; APA = Alkaline Phosphatase Activity; SOD = Superoxide dismutase activity; CAT $=$ Catalase activity; $\mathrm{d}-\mathrm{ROMs}=$ hydroperoxide $*$ Ucarr $=$ Caratelli unit (arbitrary unit) $=0.08 \mathrm{mg}$ $\mathrm{H} 2 \mathrm{O} 2 / \mathrm{dl}$ of sample; PAT $=$ plasma antioxidant potential $* * \mathrm{U}$. Cor $=$ Corelli unit (arbitrary unit) $=$ equivalent $1.4 \mu \mathrm{M}$ Ascorbic acid

Conclusion High soy protein diet in a restricted pattern affects negatively the gastrointestinal functionality in weaned piglets: increasing diarrhoea post-weaning, plasma urea nitrogen and tending to disrupt the oxidative status.

\section{References}

Lallès JP, Boudry G, Favier C, Le Floc'h N, Luron I, Montagne L, Oswald IP, Pié S, Piel C and Seve B 2004. Animal Research. 53, 301-316.

Pluske JR, Pethick DW, Hopwood DE and Hampson DJ 2002. Nutrition Research Reviews. 15, 333-371. 


\section{Satiating responses to branched chain amino acids are mediated through ileal GLP-1 secretion in post-weaning pigs}

M Muller $^{1}$, A Tilbrook ${ }^{1}$, R J van Barneveld ${ }^{2}$ and E Roura $^{1}$

${ }^{I}$ The University of Queensland, Australia, ${ }^{2}$ SunPork Group, Australia

e.roura@uq.edu.au

Application Avoiding excess dietary levels of branched chain amino acids (BCAA) may help decrease the secretion of satiety hormones and, therefore, increase feed intake in post-weaning pigs.

Introduction Previous studies have shown a negative effect of BCAA on feed intake and growth performance in young pigs when provided in high concentrations in starter diets (Wessels et al., 2016). However, the mechanisms behind their satiating effect remain unclear. Similar to what has been described in other species, we hypothesized that the satiating effect of Valine (Val), Leucine (Leu) and Isoleucine (Ile) in post-weaning pigs is related to the secretion of the anorexigenic gut peptides Cholecystokinin (CCK) and Glucagon-like peptide 1 (GLP-1).

Material and methods Six 25 day-old male pigs (Landrace x Large White) with an average weight of $6.94 \pm 0.29 \mathrm{~kg}$ were housed in pairs in an environmentally controlled room. Animals had ad-libitum access to a high-protein (24\%) diet in order to supply an adequate level of essential amino acids (AA) without adding synthetic AA. Pigs underwent an adaptation period of 3 days before being euthanized for collection of duodenum and ileum samples. Intestinal samples were stripped from their muscle layer and incubated in a 24 -well plate for $1 \mathrm{~h}$ at $37^{\circ} \mathrm{C}$ in a humidified $\mathrm{CO}^{2}$ incubator, with either glucose-free Krebs Ringer Bicarbonate (KRB) buffer containing no added AA (control) or with the addition of $10 \mathrm{mmol}$ of Leu, Ile, or Val. Following incubation, the supernatant within each well was retrieved and used for hormone analysis. A mixed analysis of variance (ANOVA) model considering AA as a fixed effect, pigs as a random effect and interactions, was used to compare the effect of each AA individually with control on CCK and GLP-1 secretion.

Results The effect of BCAA on CCK release from duodenum (A) and GLP-1 release from ileum (B) of post weaning pigs are shown below (Figure 1). GLP-1 secretion from ileum samples was significantly $(P<0.05)$ increased after Ile and Leu exposure compared to the control ( $310.68 \pm 36.33,297.44 \pm 34.42$, and $203.54 \pm 29.46 \mathrm{pmol} / \mathrm{L}$, respectively). However, only a tendency $(P>0.1)$, was observed for Val $(259.26 \pm 28.69 \mathrm{pmol} / \mathrm{L})$. Non-significant $(P>0.05) \mathrm{CCK}$ values were observed for Ile and Val compared to control $(30.36 \pm 4.77,24.17 \pm 3.62$, and $20.11 \pm 2.42 \mathrm{pg} / \mathrm{mL}$, respectively). Nonetheless, Leu resulted in a tendency $(31.68 \pm 3.57 \mathrm{pg} / \mathrm{mL}, \mathrm{P}<0.1)$ with a $57 \%$ higher CCK release than control.
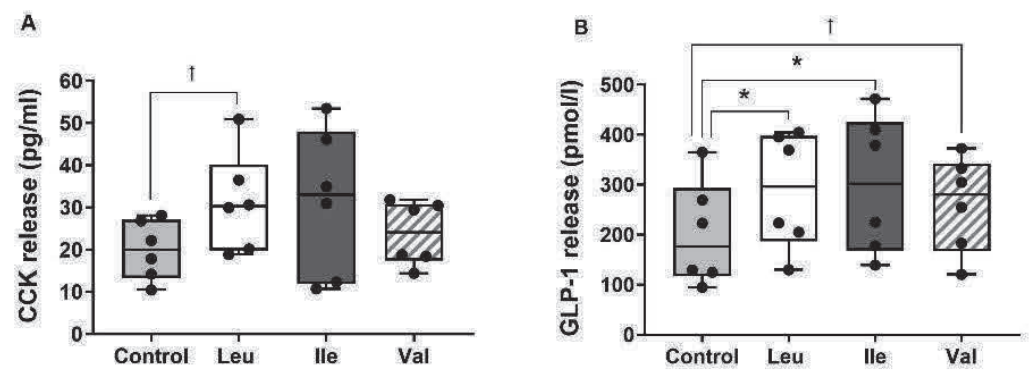

Figure 1. Duodenum CCK (A) and ileum GLP-1 (B) release following a 1-hour incubation with control (KRB buffer with no added AA), Ile, Leu or Val at $10 \mathrm{mmol} . \mathrm{n}=6$, each data point represents the average of three biological replicates within each pig. $*=P<0.05, \mathrm{t}=P<0.1$.

Conclusion The satiating effect of high dietary Ile and Leu might be mainly related to increase GLP-1 secretion from the ileum of young pigs. Excess Val did not significantly influence gut peptides from duodenum or ileum.

Acknowledgements Supported by Australian Pork Limited (APL) and The University of Queensland

\section{References}

Wessels AG, Kluge H, Hirche F, Kiowski A, Schutkowski A, Corrent E, Bartelt J, König B and Stang GI 2016. PLoS One. 11, e0150376. 


\section{Gene expression of Transient Receptor Potential Ankyrin 1 and Transient Receptor Potential} Vanilloid 1 in the gastro-intestinal tract of piglets during the peri-weaning period E Van Liefferinge ${ }^{1}, N_{\text {Van Noten }}^{1}$, J Degroote ${ }^{1}$, M Van Poucke ${ }^{1}$, L Peelman $^{1}$, E Roura $^{2}$ and J Michiels ${ }^{1}$ ${ }^{I}$ Ghent University, Belgium; ${ }^{2}$ The University of Queensland, Australia Elout.VanLiefferinge@UGent.be

Application Results will contribute to a more rational use of phytochemicals and a better understanding of the effects on gastric acid secretion, hormone release and motility.

Introduction Phytochemicals are used in animal feeds to improve nutrient digestion (Michiels et al., 2010). Several of these compounds are known to activate TRP channels. These excitatory ion channels are well described in sensory neurons and sensory terminals of taste organs, however, recent evidence indicates their expression in cells lining the GIT of mice (Camacho et al., 2015). Furthermore, it was shown that a single dose $(250 \mathrm{mg} / \mathrm{kg}$ body weight) of cinnamaldehyde, as an agonist of Transient Receptor Potential Ankyrin Channel 1 (TRPA1), could alter gastric emptying rates. Targeting TRP channels could thus be used to regulate GIT secretion and motility, yet still their expression is unknown in pig. The present study therefore investigates the potential of targeting these channels with phytochemicals, by measuring the gene expression of TRPA1 and Transient Receptor Potential Vanilloid Channel 1 (TRPV1), both known to have affinity for several phytochemicals, in different parts of the GIT in piglets pre- and post-weaning.

Material and methods Thirty-six piglets were identified from 6 litters $(\mathrm{n}=6)$. One median weight piglet from each litter group was selected for sampling at different times relative to weaning ( $23 \mathrm{~d}$ of age), $10 \mathrm{~d}$ prior (w-10d), $4 \mathrm{~h}$ post ( $\mathrm{w}+4 \mathrm{~h})$, $2,5,14$ and $28 \mathrm{~d}$ post weaning $(\mathrm{w}+2 \mathrm{~d}, \mathrm{w}+5 \mathrm{~d}, \mathrm{w}+14 \mathrm{~d}, \mathrm{w}+28 \mathrm{~d}$, respectively). Mucosal scrapings from each region of the stomach (cardia, fundus and pylorus) and SI (SI1, SI2, SI3; at 2.5, 25 and 75\% of total SI length, respectively) were collected. RT-qPCR was performed as described by Wang et al. (2016). RT-qPCR efficiencies per gene were between 97 and $102 \%$ and correlation coefficients $>0.99$. The relative expression for each gene was calculated according to the method described by Vandesompele et al. (2002), using 3 stable expressed reference genes (HPRT1, RPL4 and YWHAZ). Data were analysed using GLM procedures (SAS Institute Inc., Cary, NC, USA) with age, compartment and their interaction as fixed factors. $P$ values $<0.05$ were considered significant.

Results Both TRPA1 and TRPV1 are expressed in pig mucosa. Regarding TRPV1, age, compartment and the interaction had a significant effect on mRNA level. It increased gradually in both stomach and SI with age, though the increase was most notable in SI2 and SI3 (Figure 1). For example; $w+28 d$ piglets exhibited a significant 15- and 12-fold higher mRNA level in SI2 and SI3, respectively, as compared to newly weaned counterparts $(\mathrm{w}+4 \mathrm{~h})$. This is in contrast to TRPA1, which showed a stable expression along all time points and locations, except for a higher expression in the pyloric part at $\mathrm{w}+4 \mathrm{~h}$ (Figure 2). A 3-and 5-fold higher expression was found for $\mathrm{w}+4 \mathrm{~h}$ when compared to $\mathrm{w}-10 \mathrm{~d}$ and $\mathrm{w}+2 \mathrm{~d}$, respectively.

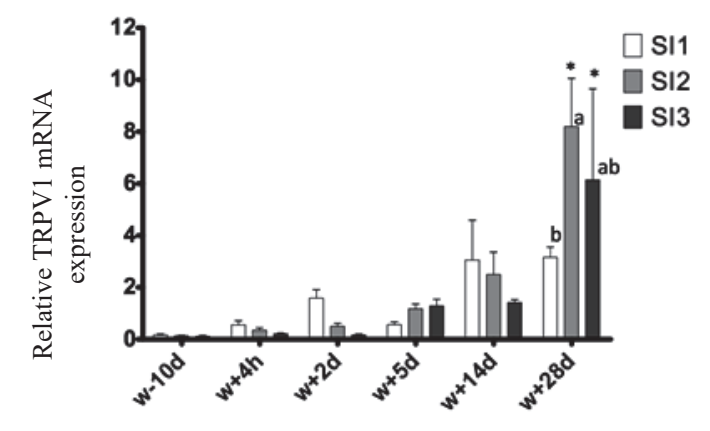

Figure 1. Relative TRPV1 mRNA level in small intestine (SI), before and after weaning $(\mathrm{w})$. *significantly different from other times $(P<0.05)$ within SI compartment. ${ }^{\mathrm{a}, \mathrm{b}}$ significantly different between compartments within same time $(P<0.05)$.

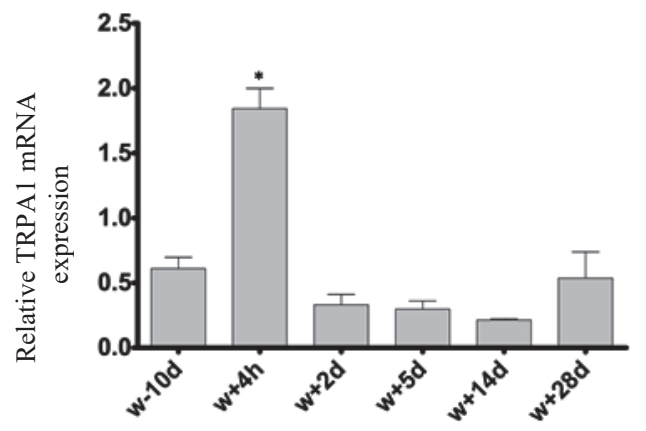

Figure 2. Relative TRPA1 mRNA level in pylorus, before and after weaning $(\mathrm{w})$. *significantly different from other times $(P<0.05)$. Error bars indicate SEM.

Conclusion Both genes are expressed in the mucosa of the piglet's GIT, which was not reported before in the case of the pig. Age has a well-defined effect on the expression of TRPV1, whereas TRPA1 is more stably expressed along all compartments with the exception of a remarkable higher expression in the pyloric region at weaning. Characterization of these channels on protein level will be necessary to localize these channels on specific enteroendocrine cell types and other GIT cell populations in order to elucidate potential functions related to GIT secretion and motility.

\section{References}

Camacho S, Michlig S, De Senarclens-Bezençon C, Meylan J and Le Coutre J 2015. Scientific Reports. 5, 7919

Michiels J, Missotten J, Van Hoorick A, Ovyn A and Dierick N 2010. Archives of Animal Nutrition. 64, 136-154.

Vandesompele J, De Preter K, Pattyn I, De Paepe A and Speleman R 2002. Genome Biology. 3, RESEARCH0034.

Wang W, Degroote J, Van Ginneken C, Dam TMT and Michiels J 2016. The FASEB Journal. 30, 863-873. 


\section{The effect of added phytase in weaner pig diets on intestinal alkaline phosphatase activity in} the mucosa of the small intestine

I Stensland $^{1}$, J C Kim ${ }^{1,2}$, J P Mansfield ${ }^{1}$, D L Turpin ${ }^{1}$, M R Bedford ${ }^{2}$ and J R Pluske ${ }^{1}$

${ }^{1}$ Murdoch University, Australia ${ }^{2}$ AB Vista, Inc., UK

ingunn.stensland@murdoch.edu.au

Application The addition of phytase in weaner diets improves zinc solubility, which will become increasingly important as pork industries worldwide implement bans on the use of pharmacological levels of zinc oxide ( $\mathrm{ZnO})$ in pig production.

Introduction Zinc oxide is a compound typically added to weaner diets at pharmacological levels to reduce post weaning diarrhoea, however there are increasing concerns and pressures around the world about its continued use due to both environmental and antimicrobial resistance issues (Pluske et al., 2018). Intestinal alkaline phosphatase (IAP) is an enzyme expressed in the mucosal layer of the gastrointestinal tract (GIT) epithelium and assists in preventing pathogen translocation and detoxification of bacterial endotoxin (LPS) (Beumer et al., 2003). Phytases, phosphorous enzymes, may increase IAP activity through increasing the availability of minerals needed for IAP expression (Martínez et al., 2004). Zinc has been showed to have an important role in this. Therefore, supplementing weaner diets with phytase, which may increase zinc availability, may be an option for preventing the decrease in IAP expression due to weaning. This experiment examined the proposition that supplementing weaner diets with phytase will increase the IAP concentration in the digesta and mucosa of the GIT.

Material and methods A total of 22 entire male pigs (Large White $\mathrm{x}$ Landrace) weighing $6.6 \pm 0.4 \mathrm{~kg}$ (mean $\pm \mathrm{SD}$ ) and weaned at $21 \mathrm{~d}$ of age were randomly allocated to one of two treatment groups $(\mathrm{n}=11)$. Diet treatments were: 1$)$ a base diet (control) and 2) control plus phytase (8 000 FTU/kg; Quantum Blue, AB Vista, Inc., UK) (Phytase). Both diets were formulated to provide $150 \mathrm{ppm} \mathrm{Zn}$ and $0.8 \%$ calcium from ingredients and premix (100 ppm $\mathrm{Zn}$ as $\left.\mathrm{ZnSO}_{4}\right)$. Both groups were offered their respective pelleted diets (15 MJ DE/kg, 1.4\% standardised ileal digestible lysine) ad libitum for $14 \mathrm{~d}$ after weaning. On d 14 and 15 pigs were euthanised and digesta samples were collected from stomach, jejunum, ileum and rectum, plus mucosal scrapings from the jejunum and ileum. The IAP activity was determined using an alkaline phosphatase assay kit according to the manufacturer's instructions (Abcam plc; Cambridge, UK). Zinc solubility in the digesta was determined as per methods described by Wang et al. (2019). Using SPSS (IBM SPSS Statistics for Windows, v24.0. Armonk, NY), mucosa and solubility data were analysed using a two-way ANOVA, there was no day of euthanasia effect. Solubility data for ileum were not normally distributed and therefore logarithmically transformed before analysis. Means were back transformed and expressed as least square means with 95\% confidence intervals (CI).

Results The measured phytase levels in the control diet and the phytase diet was $<50$ and 6540 FTU/kg of feed, respectively. The addition of phytase had no significant effect on IAP activity in the mucosa of either the jejunum (control; 1276 , phytase; 1209$)$ or ileum (control; 1019 , phytase; 947) $(P>0.05)$. An increase in zinc solubility was observed in both the jejunum (control; $16 \%$, phytase $48 \%, P<0.001$ ) and ileum (control 26\% (CI 17-35), phytase 60\%, (CI 50-69) $P$ $<0.001)$.

Conclusion The use of phytase did not cause an increase in IAP activity in the mucosa of the jejunum or ileum. Nevertheless, increases in the solubility of zinc in the digesta were observed when supplementing the diet with phytase, in agreement with previous work (Martinez et al., 2004). Future research will explore IAP gene expression to investigate in more detail these relationships.

Acknowledgements Supported in part by AB Vista and Apiam Animal Health, Portec Veterinary Services and Murdoch University for postgraduate scholarship funding.

\section{References}

Pluske JR, Turpin DL and Kim JC 2018. Animal Nutrition. 4, 187-196.

Beumer C, Wulferink M, Raaben W, Fiechter D, Brands R and Seinen W 2003. The Journal of Pharmacology and Experimental Therapeutics. 307, 737-744.

Martínez MM, Hill GM, Link JE, Raney NE, Tempelman RJ and Ernst CW 2004. The Journal of Nutrition. 134, 538544.

Wang W, Van Noten N, Degroote J, Romeo A, Vermeir P and Michiels J. 2019. Journal of Animal Physiology and Animal Nutrition. 103(1), 231-241. 


\section{Supplementation of xylo-oligosaccharides plus xylanase increased VFA and decreased BCFA production in weaned pigs}

H M Cho ${ }^{1}$, S S Wickramasuriya ${ }^{1}$, S P Macelline ${ }^{1}$, J S Hong ${ }^{1}$, Y B Kim¹ ${ }^{1}$ J C Kim² G Cordero $^{2}$, M R Bedford ${ }^{2}$ and J M $\underline{\mathrm{Heo}}^{1}$

${ }^{1}$ Chungnam National University, Republic of Korea. ${ }^{2} A B$ Vista, $U K$

jmheo@cnu.ac.kr

Application Xylo-oligosaccharides and xylanase combination can modify fermentation pattern in weaner pigs.

Introduction The reduction of antimicrobial growth promotors (AGP) increases pathogenic bacterial challenge in weaned pigs particularly in a poorer sanitary environment. Previous reports have indicated that dietary xylo-oligosaccharides (XOS) stimulates beneficial bacteria in the intestinal tract enhancing the immunity of weaner pigs (Liu et al., 2018). The hypotheses tested in this study were (1) pigs housed in a poorer sanitary condition will increase pro-inflammatory cytokine production, and (2) combined supplementation of xylo-oligosaccharides (XOS) and xylanase will reduce proinflammatory cytokine production via stimulation of fibre fermenting microbiota when compared to a mannanoligosaccharide (MOS) or fructo-oligosaccharide (FOS).

Materials and methods A total of 144 male pigs [Duroc $\mathrm{x}$ (Yorkshire $\mathrm{x}$ Landrace)] weaned at $28 \mathrm{~d}$ of age with initial body weight $(\mathrm{BW})$ of $7.5 \pm 0.7 \mathrm{~kg}$ (mean \pm SEM) were randomly allocated to six dietary treatments [Sanitary condition: control (CON; $<150$ ppm ZnO), CON with XOS plus xylanases (CXX; Signis, AB Vista, Marlborough, UK; 100 g/t); Poorer sanitary condition (without cleaning/disinfection of a previously populated room) : CON, CXX, CON with MOS (CM; Bio-Mos ${ }^{\circledR}$, Alltech Inc, Nicholasville, KY, USA; 1000 g/t), CON with FOS (CF; FOS-MAX ${ }^{\circledR}$, Dreamfeed Inc., Seoul, Republic of Korea; $2000 \mathrm{~g} / \mathrm{t}$ )] to give 6 replicates per treatment with 4 pigs per pen. All diets were formulated base on corn, wheat and soybean meal to meet nutrition specification (NRC, 2012). Blood samples $(5-10 \mathrm{~mL}$ ) and faecal samples were collected from 6 selected pigs per treatment (closest to the median BW) on d 14. The concentrations of interleukin 1 $\beta, 6$ (IL-1 $\beta$, IL-6; R\&D Systems, Minneapolis, MN, USA), tumour necrosis factor $\alpha$ (TNF- $\alpha$; R\&D Systems, Minneapolis, MN, USA) in plasma were quantified using commercially available ELISA kits. Faecal samples were analysed for volatile fatty acids (VFA) including branched chain fatty acids (BCFA). Data were analysed as a completely randomized block design using general linear model procedure of ANOVA for environmental effect and diet effect under poorer sanitary condition.

Results Housing piglets in poorer sanitary condition increased TNF- $\alpha$ production by $90 \%(P<0.001)$ and supplementation of CXX, FOS and MOS did not reduce TNF- $\alpha$ production. Supplementation of CXX increased total VFA production $(26 \%$ improvement under poor unsanitary; $P<0.001)$ and reduced BCFA production $(14 \%$ and $60 \%$ reduction under sanitary and poor sanitary condition, respectively; $P<0.001$ ). The interaction between sanitary condition and diet $(P<0.001)$ indicated that a diet effect on VFA and BCFA is more obvious in piglets housed under poor sanitary conditions.

Table 1. Impact of sanitary conditions and dietary treatments (control, CON; control with xylo-oligosaccharide plus xylanases, CXX; control with mannan-oligosaccharides, CM; control with fructo-oligosaccharides, $\mathrm{CF}$ ) on volatile fatty acid (VFA) and cytokine contents (interleukin 1 $\beta$, IL-1 $\beta$; interleukin 6, IL-6; tumour necrosis factor $\alpha$, TNF- $\alpha$ ) measured at day 14 after weaning.

\begin{tabular}{lcccccccccc}
\hline Sanitation & \multicolumn{2}{c}{ Sanitary } & \multicolumn{4}{c}{ Poorer Sanitary } & \multirow{2}{*}{ SEM } & \multicolumn{3}{c}{$P$ value $^{1}$} \\
Diet & CON & CXX & CON & CXX & CM & CF & & CXX & E $\times$ CXX & DPS \\
\hline Total VFA $(\mathrm{mg} / \mathrm{l})$ & $148^{\mathrm{b}}$ & $153^{\mathrm{b}}$ & $130^{\mathrm{c}}{ }_{\mathrm{y}}$ & $164^{\mathrm{a}}{ }_{\mathrm{x}}$ & $153^{\mathrm{b}}{ }_{\mathrm{x}}$ & $153^{\mathrm{b}}{ }_{\mathrm{x}}$ & 1.9 & $<0.001$ & $<0.001$ & $<0.001$ \\
BCFA (\%) & $10.9^{\mathrm{b}}$ & $9.6^{\mathrm{c}}$ & $13.1^{\mathrm{a}}{ }_{\mathrm{x}}$ & $8.2^{\mathrm{d}}{ }_{\mathrm{z}}$ & $9.1^{\text {cd }}$ & $9.2^{\text {cd }}$ & 0.13 & $<0.001$ & $<0.001$ & $<0.001$ \\
IL-1 $\beta(\mathrm{pg} / \mathrm{mL})$ & 21.7 & 19.8 & 31.6 & 24.6 & 26.8 & 27.5 & 5.12 & 0.962 & 0.790 & 0.970 \\
IL-6 $(\mathrm{pg} / \mathrm{mL})$ & 47.0 & 38.3 & 82.6 & 57.4 & 70.3 & 68.9 & 10.26 & 0.554 & 0.653 & 0.860 \\
TNF- $\alpha(\mathrm{pg} / \mathrm{mL})$ & $5.4^{\mathrm{bc}}$ & $5.2^{\mathrm{c}}$ & $10.2^{\mathrm{a}}$ & $7.8^{\mathrm{abc}}$ & $8.6^{\mathrm{ab}}$ & $8.3^{\mathrm{abc}}$ & 0.51 & 0.008 & 0.282 & 0.359 \\
\hline
\end{tabular}

a,b,c,d Means within a row with different superscripts differ significantly in dietary treatments $(P<0.05)$; ${ }^{\mathrm{x}, \mathrm{y}, \mathrm{z} \text { Means }}$ within a row with different subscripts differ in diets under poorer sanitary $(P<0.05)$; SEM, standard error of mean (for poorer sanitary); E, environment; DPS, diet effect under poorer sanitary; BCFA (branched chain fatty acids), molar proportion of isobutyic acid, isovaleric acids and valeric acid with respect to the total VFA. Environment effect was not significant $(P>0.05)$ except TNF- $\alpha(P<0.001)$.

Conclusion Results indicate that housing pigs under poorer sanitary conditions increases the inflammatory response. Supplementation of CXX, CF and CM could not completely ameliorate this response but the combined supplementation of CXX resulted in better fermentation and inflammatory characteristics than CF or CM alone when piglets were housed under poorer sanitary conditions.

Acknowledgements This study was financially supported by AB Vista (Marlborough, UK)

\section{Reference}

Liu JB, Cao SC, Liu J, Xie YN and Zhang HF 2018. Asian-Australasian Journal of Animal Sciences. $31,1660$.

NRC 2012. Nutrient Requirements of Swine: $11^{\text {th }}$ Revised Edition. The National Academies Press, Washington DC, USA. 


\section{Effects of glucocorticoid receptor agonist on apparent ileal and total tract digestibility of dietary nutrients and the histomorphology of the small intestine in newly weaned pigs}

H Wooten ${ }^{1}$, A R Rakhshandeh ${ }^{2}$ and A Rakhshandeh ${ }^{1}$

${ }^{1}$ Texas Tech University, USA, ${ }^{2}$ South Plains College, USA

anoosh.rakhshandeh@ttu.edu

Application The purpose of this study is to better understand the mechanism through which a glucocorticoid receptor agonist, an alternative to in-feed antibiotics, improves the productivity of newly weaned pigs.

Introduction In previous studies, we observed that a glucocorticoid receptor agonist (GRA) mitigated the negative effects of systemic inflammation in early weaned pigs (Wooten et al., 2019b). It also improved the intestinal integrity (at the transcriptional level) and overall immune function, which led to improved growth performance during the starter phase (Wooten et al., 2019a,b). Apparent ileal digestibility (AID) of dietary crude protein (Cp), apparent total tract digestibility (ATTD) of dietary dry matter (DM) and histomorphology of the jejunum were evaluated to better understand the mechanisms through which GRA improves the productivity of piglets.

Material and methods Two hundred and nine PIC piglets $(7 \pm 2.1 \mathrm{~kg})$ weaned at $26 \pm 1.5 \mathrm{~d}$ of age were blocked by time and assigned to eight treatments (5-7 piglets/pen; 4 pens/treatment) in a 2 x 4 factorial arrangement. The main effects were i) sex (barrow and gilts) and, ii) four pharmaceutical treatments (i.m. injection of saline, CON; i.m. injection of GRA; in-feed antibiotic, ANT; and antibiotic plus GRA, ANGA). Dexamethasone (two injections of $0.2 \mathrm{mg} / \mathrm{kg}$ body weight, given at $-24 \mathrm{~h}$ and $48 \mathrm{~h}$ post-weaning) and Tylosin (110 ppm for $7 \mathrm{~d}$ ) were used as the GRA and the in-feed antibiotic. Pigs were fed a corn soy-based diet according to a phase-feeding program (NRC, 2012). One hundred and twenty piglets were euthanized via lethal injection with sodium pentobarbital on $\mathrm{d} 1,3$ and 5 post-weaning (15 pigs/ treatment; 5 pigs/d) and ileal digesta was collected for AID measurements. A segment of the jejunum was excised and used for histomorphological evaluations. Faecal samples were collected weekly for four weeks and used to determine ATTD of dietary DM. Statistical analysis was performed with sex, pharmaceutical treatment, time (day), the interaction and block as fixed effects and pen within treatment as a random effect. Mixed model repeated measure analysis of variance was used to compare the treatment means (SAS Institute Inc., Cary, NC, USA).

Results No interaction among the main effects and time on AID of dietary Cp was observed. Relative to CON, GRA increased the ATTD and AID of dietary DM and Cp, respectively (Table 1). No differences between the effects of ANT and GRA on nutrient digestibility were observed. Villus height and the villus height-to-crypt depth ratio were higher in the ANT and CON groups, compared to the GRA group. No treatment effects were observed on villus density.

Table 1. Effect of pharmaceutical treatment of weaned piglets (saline control, CON; glucocorticoid receptor agonist, GRA; in-feed antibiotic, ANT; or in-feed antibiotic plus GRA, ANGA) on measures of digestibility (apparent total tract digestibility of dietary dry matter, ATTD of DM; apparent ileal digestibility of dietary crude protein, AID of Cp) and jejunum intestinal mucosa histomorphology.

\begin{tabular}{lcccccc}
\hline & CON & GRA & ANT & ANGA & SE & $P$ value \\
\hline Nutrient digestibility & & & & & & \\
ATTD of DM & $0.90^{\mathrm{b}}$ & $0.94^{\mathrm{a}}$ & $0.93^{\mathrm{a}}$ & $0.93^{\mathrm{a}}$ & 0.010 & 0.01 \\
$\quad$ AID of Cp & $0.87^{\mathrm{b}}$ & $0.90^{\mathrm{a}}$ & $0.90^{\mathrm{a}}$ & $0.87^{\mathrm{ab}}$ & 0.019 & 0.02 \\
Histomorphology & & & & & & \\
$\quad$ Villus height, $\mu \mathrm{m}$ & $0.26^{\mathrm{a}}$ & $0.24^{\mathrm{c}}$ & $0.27^{\mathrm{a}}$ & $0.25^{\mathrm{a}}$ & 0.010 & 0.02 \\
Crypt depth, $\mu \mathrm{m}$ & 0.23 & 0.26 & 0.25 & 0.24 & 0.010 & 0.08 \\
Villus density, villi/mm & 9.29 & 9.35 & 9.41 & 9.11 & 0.220 & 0.78 \\
Villus height: crypt depth & $1.15^{\mathrm{a}}$ & $0.96^{\mathrm{b}}$ & $1.13^{\mathrm{a}}$ & $1.09^{\mathrm{ab}}$ & 0.054 & 0.02 \\
\hline a,b,c Means within a row with different superscripts differ significantly $(P<0.05) ;$ SE, standard error
\end{tabular}

Conclusion Our results demonstrate that GRA increases the AID and ATTD of dietary nutrients, compared to control pigs. However, GRA does this by most likely mitigating the negative effects of inflammation, rather than improving villus architecture. Thus, GRA treatment may result in improved postabsorptive nutrient utilization that is comparable to treatment with subtherapeutic antibiotics.

Acknowledgements Supported in part by USDA-NIFA 2017-68008-26304.

\section{References}

NRC 2012. Nutrient Requirements of Swine: $11^{\text {th }}$ Revised Edition. The National Academies Press, Washington DC, USA. Wooten H, Garcia A, McGlone JJ, Rakhshandeh AR and Rakhshandeh A (2019a). Journal of Animal Science. 97 (Suppl.2), 46-47.

Wooten H, McGlone JJ, Wachtel M, Thompson G, Rakhshandeh AR and Rakshandeh A (2019b). Animal. $10,1-10$. 


\section{Effect of glucocorticoid administered to sows at farrowing on piglet performance}

\section{S A Ward ${ }^{1}$, R N Kirkwood ${ }^{1}$, D S Lines ${ }^{2}$, K J Plush ${ }^{2}$}

${ }^{I}$ The University of Adelaide, Australia, ${ }^{2}$ SunPork Group, Australia

sophia.ward@adelaide.edu.au

Application When inducing sows to farrow, the provision of dexamethasone 24 hours after induction has the potential to improve piglet preweaning survival. A major driver of profit in the swine industry is the number of pigs marketed per sow per year, (Tokach et al., 2016) so this protocol shows promise from both an economic and welfare perspective.

Introduction The farrowing period is a time of great risk to the neonate, and analgesia may allow sows to cope with farrowing and/or lactation processes more effectively. In contrast to non-steroidal anti-inflammatories, glucocorticoids (GCs) diffuse across cell membranes to stimulate anti-inflammatory responses directly within the tissue. As well as treating inflammatory pain, administering GCs in late gestation has been found to prompt the maturation of visceral organs in offspring of a variety of mammalian species (Aghajafari et al., 2002). The aim of this study was to assess the effect of GCs on piglet performance, with the hypothesis that an optimum timing of administrating GCs in relation to parturition exists.

Material and methods Sows were induced to farrow with a split-dose cloprostenol injection the day before their due date and randomly allocated to receive a $20 \mathrm{mg}$ dose of dexamethasone either with induction (DexInd; $\mathrm{n}=20$ ), $24 \mathrm{~h}$ after induction (Dex24h; $n=20$ ), with induction and $24 \mathrm{~h}$ after (Dexboth; $\mathrm{n}=19$ ) or no treatment (Control; $\mathrm{n}=20$ ). At d 2 a blood sample was assayed for total protein in plasma (\%) using Brix refractometry as an indicator of colostrum ingestion. Piglet weights at d 1, 2 and 18, and all pig mortalities were recorded. Data were analysed using a linear mixed model with the random terms replicate, room and sow identification, and the main fixed effects of gender and treatment (IBM SPSS Statistics for Windows, v20.0. Armonk, NY). Litter size and sow parity were fit as covariates.

Results Piglets born to Dexboth and DexInd sows had significantly lower d 1 and 2 weights and tended to have reduced d 18 weights (Table 1). Piglets from Dex24h sows had the lowest incidence of piglet mortality to weaning. Dex 24 and Control sows had piglets with higher $24 \mathrm{~h}$ serum protein $(\%)$.

Table 1. The effect (mean \pm SEM) of treatment with dexamethasone (Control, no treatment; DexInd, a single $20 \mathrm{mg}$ dose at induction; Dexboth, a double $20 \mathrm{mg}$ dose at and $24 \mathrm{~h}$ after induction; Dex $24 \mathrm{~h}$, a single $20 \mathrm{mg}$ dose $24 \mathrm{~h}$ after induction) on the performance of piglets.

\begin{tabular}{lccccc}
\hline & Control & DexInd & Dexboth & Dex24h & $P$ value \\
\hline d 1 piglet weight $(\mathrm{kg})$ & $1.37^{\mathrm{a}} \pm 0.02$ & $1.28^{\mathrm{b}} \pm 0.02$ & $1.24^{\mathrm{b}} \pm 0.02$ & $1.38^{\mathrm{a}} \pm 0.02$ & 0.001 \\
d 2 piglet weight $(\mathrm{kg})$ & $1.55^{\mathrm{a}} \pm 0.02$ & $1.46^{\mathrm{c}} \pm 0.02$ & $1.40^{\mathrm{b}} \pm 0.02$ & $1.54^{\mathrm{a}} \pm 0.02$ & 0.001 \\
$\mathrm{~d} 18$ piglet weight $(\mathrm{kg})$ & $5.83^{\mathrm{a}} \pm 0.08$ & $5.52^{\mathrm{b}} \pm 0.08$ & $5.47^{\mathrm{b}} \pm 0.8$ & $6.00^{\mathrm{a}} \pm 0.09$ & 0.060 \\
24 h serum protein $(\%)$ & $6.8^{\mathrm{a}} \pm 0.1$ & $6.2^{\mathrm{b}} \pm 0.1$ & $6.2^{\mathrm{b}} \pm 0.1$ & $6.9^{\mathrm{a}} \pm 0.1$ & 0.003 \\
Incidence of piglet mortality & $0.12^{\mathrm{a}} \pm 0.02$ & $0.12^{\mathrm{a}} \pm 0.01$ & $0.13^{\mathrm{a}} \pm 0.02$ & $0.08^{\mathrm{b}} \pm 0.01$ & 0.044 \\
\hline \multicolumn{5}{c}{ a,b,c Means within a row with different superscripts differ significantly $(P<0.05)$}
\end{tabular}

Conclusion Administering dexamethasone with induction affects piglet weight from birth to weaning and is not an effective protocol for improving piglet performance. Further research should focus on administering dexamethasone 24 hours after induction as it did not negatively affect piglet weights and has the potential to improve piglet survival to weaning.

Acknowledgements Supported in part by Australian Pork Limited and The University of Adelaide.

\section{References}

Aghajafari F, Murphy K, Matthews S, Ohlsson A, Amankwah K and Hannah M 2002. American Journal of Obstetrics and Gynecology. 186, 843-849.

Tokach MD, Goodband BD and O’Quinn TG 2016. Animal Frontiers. 4, 15-21. 


\section{Non-linear associations were found between litter size and sow survival to the next parity}

$\mathrm{N}$ Kerssen $^{1,2}$ and $\underline{\mathrm{S} \mathrm{Hermesch}}^{1}$

${ }^{1}$ Animal Genetics and Breeding Unit, a joint venture of NSW Department of Primary Industries and University of New England, University of New England, Australia, ${ }^{2}$ Animal Breeding and Genomics, Wageningen University \& Research, the Netherlands.

Susanne.Hermesch@une.edu.au

Application Number of piglets born alive showed positive or no phenotypic associations with sow survival to subsequent parities. This implies that high litter sizes did not have detrimental effects on sow longevity, and both can be improved.

Introduction Improving sow longevity is necessary for both economic importance and welfare concerns. But, sow longevity is a difficult trait to select for because it can only be measured at the end of a sow's life. Number born alive (NBA) was positively associated with sow longevity and it was suggested as an early indicator trait for sow longevity (Engblom et al., 2016). However, non-linear associations between NBA and sow survival were not explored. This study aimed to investigate whether a larger NBA continuously favoured sow survival to subsequent parities.

Material and methods Data included 2190 Large White (LW) and 1685 Landrace (LR) sows from one herd with at least 1 parity recorded. Birth years were from 2006 to 2016, and all sows had the opportunity to reach at least parity 4. Data were therefore not censored. Number of piglets born alive was grouped when the number was 5 or less and 16 or more. Binomial generalized linear mixed models were used to test significance of fixed effects for the survival rates of sows to the subsequent parity up to parity 4 (SAS Institute Inc., Cary, NC, USA). Farrowing year-season (3-month period) and NBA in the previous parity (e.g. 1, 2 and 3 ) were significant fixed effects $(P<0.001)$ for survival rates of sows which were analysed separately for each parity and breed. Additional analyses combining both breeds showed that breed was only significant for survival rate to parity 2 and there was no breed by NBA interaction. To display the results, Least Squares Means of the survival rates from parity 1 to 2, 2 to 3 and 3 to 4 were visualised on the observed scale (Figure 1).

Results The percentages of sows that survived to a next parity were 79.0, 75.4 and 74.9\% for LW and $61.7,68.8$ and $70.6 \%$ for LR sows. In both breeds, sows with larger litter sizes had higher survival rates to the next parity (Figure 1). Survival rates of sows increased with larger NBA until 11 piglets in both breeds. The survival rates for the levels below 11 piglets born alive mainly reflect management decisions, since litter size in maternal sow lines is an important production trait. In general, sows with low litter sizes are not retained for the next parity. The survival rates for the levels above 11 piglets born alive indicate that sows did not have decreased survivability when farrowing large litters, confirming the findings by Koketsu (2007) for high and low productivity herds that large litter sizes were not in conflict with better longevity of sows. Finally, survival rates of LW sows to parity 2 were higher for low NBA values in comparison to later parities, however, these differences were not significant.
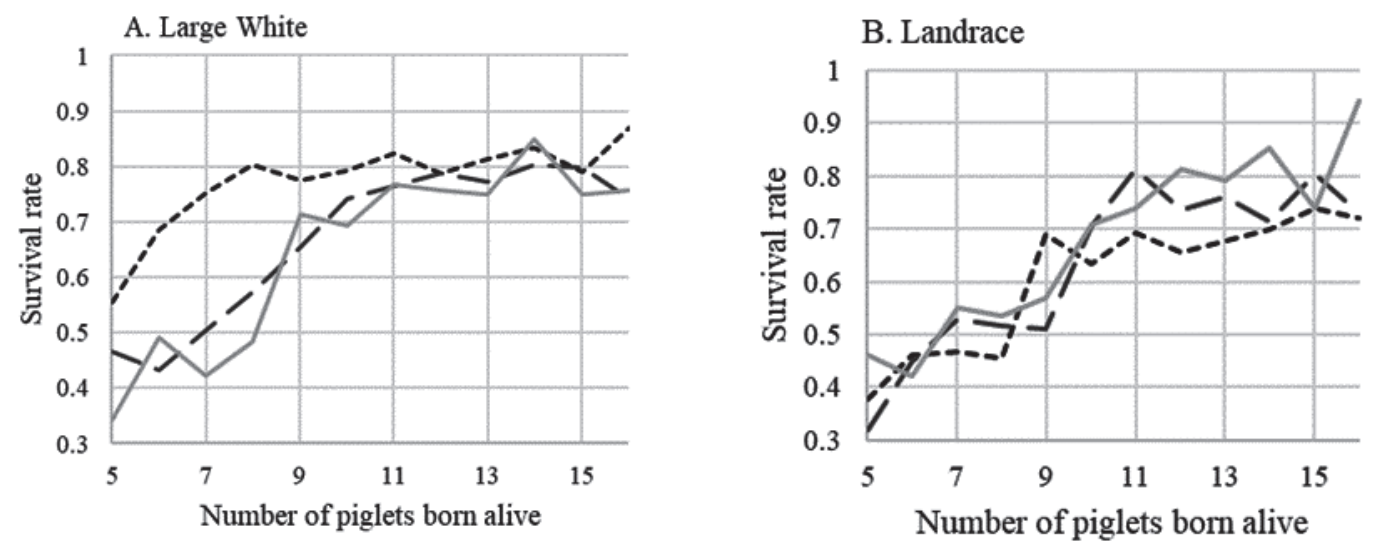

Figure 1. Survival rates to the next parity (2, 3 and 4) for Large White (A, LW) and Landrace (B, LR) per number of piglets born alive in litter 1 (dotted line), 2 (dashed line) and 3 (solid grey line). Standard errors of Least Squares Means varied from 0.02 to 0.09 in LW and from 0.03 to 0.12 in LR.

Conclusion Survival rates of sows to the next parity increased with higher NBA in the first three parities until 11 piglets and were not affected by NBA levels above 11 piglets.

\section{References}

Engblom L, Calderón Díaz JA, Nikkilä M, Gray K, Harms P, Fix J, Tsuruta S, Mabry J and Stalder KJ 2016. Journal of Animal Breeding and Genetics. 133, 138-144.

Koketsu Y 2007. Journal of Animal Science. 85, 1086-1091. 


\title{
Differences in farrowing rate according to parity and trait specific temperature groupings around mating events
}

\author{
A M G Bunz $^{1,2}$, K L Bunter ${ }^{2}$, R S Morrison ${ }^{1}$, B G Luxford ${ }^{1}$ and S Hermesch ${ }^{2}$ \\ ${ }^{1}$ Rivalea Australia Pty Ltd, Australia, ${ }^{2}$ Animal Genetics and Breeding Unit, a joint venture of NSW Department of \\ Primary Industries and University of New England, University of New England, Australia \\ abunz@rivalea.com.au
}

Application Temperature groupings quantify the effects of different temperature patterns around mating on farrowing rate. Providing better cooling systems in the farrowing house could possibly reduce the reduction in farrowing rate seen in this study, especially for matings of parity 1 sows.

Introduction A methodology has been developed using cluster analysis to define trait-specific temperature groupings (Bunz et al., 2019). These temperature groupings accounted for the different maximum temperature (Tmax) histories sows were exposed to around first mating, independently of calendar months. It is hypothesized, that temperature groupings have an impact on farrowing rate and that there are significant differences in farrowing rate between mating parities in temperature grouping.

Material and Methods Information about 36767 first-insemination farrowing rate (FR) records of 17090 parity 0,1 and 2 sows collected from 2012 to 2017 from a single farm in southern New South Wales, Australia were available. Sows were housed in naturally ventilated sheds and had drip cooling provided during their lactation period when shed temperature exceeded $30^{\circ} \mathrm{C}$. A generalized linear model with a logit link function was applied to the data. The model included mating parity, line (Large White, Landrace and Duroc, Primegro ${ }^{\mathrm{TM}}$ Genetics, Corowa, NSW), temperature grouping (T-group) relevant to mating date, year-quarter of mating date, mating parity by T-group interaction and line by T-group interaction as predictors for FR (R Core Team, 2018). The following steps outlined further in Bunz et al. (2019) were applied for defining 5 T-groups $(\mathrm{n}=5)$ : a) a generalized linear model with a logit link was used to identify significant days $(P<0.05)$ for FR at first insemination regarding daily maximum ambient temperature (Tmax) in the time period 35 days prior to and 35 days post mating date; b) for every mating date the Tmax of significant days were extracted; and, c) a cluster approach based on partitioning around medoids (PAM; Kaufmann and Rousseeuw, 1990) methods was applied to group temperature patterns for every mating date according to their similarity.

Results Mating parity, line, T-group and mating year quarter were significant $(P<0.001)$ for the prediction of farrowing rate at first insemination. Interactions between line and T-group $(P<0.05)$ and parity by T-group $(P<0.05)$ were also evident. High temperatures prior to mating caused the largest reduction in predicted farrowing rate across all mating parity groups (Table 1). Mating parity one had the greatest reduction in predicted FR when maximum ambient temperature was high $\left(>29^{\circ} \mathrm{C}\right)$ before the mating event.

Table 1. Mean predicted farrowing rate $(\%) \pm$ SEM of the prediction according to temperature grouping (T-group) and

\begin{tabular}{|c|c|c|c|c|c|}
\hline \multirow[t]{2}{*}{ T-group } & \multirow{2}{*}{$\begin{array}{l}\text { Mean maximum temperature } \\
\text { characteristic of T-group }^{1}\end{array}$} & \multirow{2}{*}{$\begin{array}{l}\text { Number of } \\
\text { records }\end{array}$} & \multicolumn{3}{|c|}{ Mating parity } \\
\hline & & & 0 & 1 & 2 \\
\hline 1 & high prior and post mating & 8686 & $79.5^{\mathrm{a}} \pm 0.84$ & $75.0^{\mathrm{b}} \pm 1.00$ & $77.6^{\mathrm{a}} \pm 1.03$ \\
\hline 2 & low prior and medium post mating & 6989 & $84.2^{\mathrm{a}} \pm 0.82$ & $79.9^{b} \pm 1.05$ & $81.9^{\mathrm{ab}} \pm 1.07$ \\
\hline 3 & high prior and medium post mating & 5471 & $81.1^{\mathrm{a}} \pm 0.93$ & $72.1^{\mathrm{b}} \pm 1.21$ & $78.5^{\mathrm{a}} \pm 1.22$ \\
\hline 4 & medium prior and low post mating & 5204 & $82.6^{\mathrm{a}} \pm 1.04$ & $82.6^{\mathrm{a}} \pm 1.10$ & $84.2^{\mathrm{a}} \pm 1.15$ \\
\hline 5 & low prior and post mating & 10411 & $85.9^{\mathrm{a}} \pm 0.67$ & $83.5^{\mathrm{a}} \pm 0.81$ & $84.4^{\mathrm{a}} \pm 0.86$ \\
\hline
\end{tabular}

${ }^{\mathrm{ab}}$ Means within a row with different superscripts differ significantly $(P<0.05) ;{ }^{1}$ Mean max temperature characteristics: high $>29^{\circ} \mathrm{C}$; medium $21-29^{\circ} \mathrm{C}$; low $<21^{\circ} \mathrm{C}$

Conclusion Significant differences occurred in FR from the first insemination event for different T-groups and parities. This suggests that the mechanism involved with heat stress differs between the three mating parity groups. Gourdine et al. (2017) found a greater thermal response to heat stress in lactating primiparous compared to multiparous sows, which is consistent with the largest reduction in FR for sows in mating parity 1 observed in this study. The larger reduction in parity 1 sows is may associated with higher heat stress during lactation.

Acknowledgements Supported in part by Australian Pork Limited (Project number 2017/2208 and Student scholarship 2018/007).

\section{References}

Bunz AMG, Bunter KL, Morrison RS, Luxford BG and Hermesch S. 2019. Advances in Animal Biosciences. 10 , s66.

Gourdine JL, Mandonnet N, Giorgi M and Renaudeau D. 2017. Animal. 11, 365-374.

Kaufman L and Rousseeuw PJ 1990. Finding groups in data: an introduction to cluster analysis. pp. 68-107. John Wiley \& Sons, Hoboken, NJ, USA.

R Core Team 2018. R: A language and environment for statistical computing. R Foundation for Statistical Computing, Vienna, Austria. 


\section{A comparison of different physiological temperature measurements of lactating pure and crossbred lines sows over summer}

$\underline{\text { S L Beer }}{ }^{1}$, F Liu ${ }^{1}$ and S Smith ${ }^{1}$

${ }^{1}$ Rivalea Australia Pty Ltd, Australia

sbeer@rivalea.com.au

Application Crossbred sows (Line A x Line B) had significantly lower rectal temperature than pure Line A sows during a lactation period with a daily average maximum ambient temperature above $34^{\circ} \mathrm{C}$. This difference in breed response may indicate the cross bred sows cope better under these conditions.

Introduction Heat stress has been observed to impact sows' productivity and welfare particularly during lactation (Parois et al., 2017). This has impacts on pig farm productivity and increases variability in litters raised by sows during conditions of high ambient temperatures. The results of a previous study by Gourdine et al. (2007) indicates that some breeds have a better tolerance to heat stress than others. However, there are limited reports in literature of physiological response to high ambient temperature between pure and crossbred sows in lactation due to the irregularity of these animals being housed together. Therefore, the aim of this study is to evaluate physiological temperature measurements of lactating pure and crossbred line sows over summer when housed in a commercial environment. It is hypothesised that there are differences in thermal response between pure and crossbred lactating sows over summer.

Material and methods Thermal physiological measurements were collected from parity 2 and 3 pure and crossbred line sows (Landrace-Line A ( $\mathrm{n}=35)$, Large White-Line $\mathrm{B}(\mathrm{n}=35)$ and Large White $\mathrm{x}$ Landrace $(\mathrm{n}=33)$, Primegro ${ }^{\mathrm{TM}}$ Genetics, Corowa, NSW) during lactation. Sows were housed in naturally ventilated sheds and drip cooling system provided, as temperature exceeded $30^{\circ} \mathrm{C}$. Sows in each of the three replicates were measured for thermal physiological responses on the same calendar day at the average $\mathrm{d} 3 \pm 1.1$ (mean \pm standard deviation (SD)), d $15 \pm 1.3$ and $\mathrm{d} 21 \pm 1.1$ of lactation. The average lactation days on each monitoring day point were similar among the breeds. The thermal physiological measurements collected were respiration rate (RR), rectal temperature (RT), eye temperature (EYE) and skin temperature (SKIN). Skin and eye temperature were collected using an FLIR thermal imaging camera (emissivity $=0.98$, FLIR Systems Inc., Tallin, Estonia), with skin temperature being the hottest point of the sows' shoulder (clean and dry) and eye temperature the centre of the sows' open eye. Rectal temperature was measured using a digital thermometer and respiration rate was counted as breaths/minute. The study was conducted over summer months from January to February 2019 and the average daily maximum temperature was $34.5^{\circ} \mathrm{C}$. The in-shed temperature during the physiological monitoring $\left(1500-1700 \mathrm{~h}\right.$ ) was $33.5 \pm 4.52^{\circ} \mathrm{C}, 28.1 \pm 4.49^{\circ} \mathrm{C}$, and $32.6 \pm 4.58^{\circ} \mathrm{C}$ (mean $\pm \mathrm{SD}$ ) for d 3,15 and 21 , respectively. A linear mixed model with line, lactation week, environmental group (replicate) and parity as fixed effects and sow as a random effect (R Core Team 2013) was used to quantify the differences between lines in physiological measurements (Table 1).

Results Parity was only included in the model for RR as this was the only measurement where it was found to have significant effect. Environmental group and lactation week were significant for all physiological temperature measurements. Line was only significant for rectal temperature $(P=0.023)$. Specifically, the rectal temperature of Line A sows was $0.29^{\circ} \mathrm{C}$ greater than the crossbred sows $(P=0.009)$.

Table 1. Physiological temperature measurements and respiration rate (LSM $\pm \mathrm{SE}$ ) of lactating sows over summer in

\begin{tabular}{lccccc}
\multicolumn{6}{c}{ pure and crossbred lines } \\
\hline Line & n. Sows & $\begin{array}{c}\text { Rectal } \\
\left({ }^{\circ} \mathrm{C}\right)\end{array}$ & $\begin{array}{c}\text { Respiration Rate } \\
(\text { breaths } / \text { min })\end{array}$ & $\begin{array}{c}\text { Eye } \\
\left({ }^{\circ} \mathrm{C}\right)\end{array}$ & $\begin{array}{c}\text { Skin } \\
\left({ }^{\circ} \mathrm{C}\right)\end{array}$ \\
\hline $\mathrm{A}$ & 35 & $39.32^{\mathrm{b}} \pm 0.07$ & $54.8 \pm 2.87$ & $37.64 \pm 0.09$ & $37.96 \pm 0.09$ \\
$\mathrm{~B}$ & 35 & $39.11^{\mathrm{ab}} \pm 0.07$ & $51.8 \pm 2.85$ & $37.50 \pm 0.09$ & $37.92 \pm 0.09$ \\
$\mathrm{~A} \times \mathrm{B}$ & 33 & $39.04^{\mathrm{a}} \pm 0.07$ & $48.0 \pm 2.87$ & $37.58 \pm 0.09$ & $37.72 \pm 0.10$ \\
$P$ value & & 0.023 & 0.23 & 0.52 & 0.17 \\
\hline
\end{tabular}

a,b Means within a column with different superscripts differ significantly $(\mathrm{P}<0.05)$

Conclusion It was observed that the rectal temperature of crossbred sows was significantly lower than the pure Line A sows during summer lactation. Whether this was due to a greater thermoregulatory ability of the cross bred sows remains to be further investigated. In addition, it is of potential interest to study whether the lactation performance and subsequent reproductive performance of the crossbred sows are more thermal tolerant than the pure line A.

Acknowledgements Supported by Research and Innovation Unit and Farming Operations at Rivalea Australia Pty Ltd

\section{References}

Gourdine J, Bidanel J, Noblet J and Renaudeau D 2007. Asian-Australian Journal of Animal Science. 20, $832-841$. Parois S, Cabezon F, Schinckel A, Johnson J, Stwalley RM and Marchant-Forde J 2017. Pig Welfare Symposium, Des Moines, IA, USA.

R Core Team 2013. R: A language and environment for statistical computing. R Foundation for Statistical Computing, Vienna, Austria. 


\section{Heat stress during mid-gestation up-regulates placental insulin-like growth factor 2 (IGF-2)} mRNA expression and affects hormonal profiles in pregnant gilts

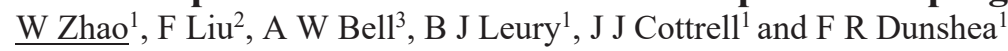

${ }^{1}$ The University of Melbourne, Australia, ${ }^{2}$ Rivalea Australia Pty Ltd, Australia, ${ }^{3}$ Cornell University, USA

weichengz@student.unimelb.edu.au

Application Heat stress during mid-gestation up-regulated placental insulin-like growth factor 2 (IGF-2) mRNA relative expression and altered gilt circulating endocrine levels, which may be an adaptation to compromised placental function.

Introduction Seasonal infertility occurs when summer conditions collectively reduce sow fertility. A further concern with seasonal infertility is the effect of gestational heat stress (GHS) on placental efficiency (foetal:placental weight ratio), which is a causative factor for altered foetal development (Bell et al., 1989). We have previously demonstrated that GHS increased placental weights and size while reducing placental efficiency (Zhao et al., 2019). This placental insufficiency may be due to changes in mediators that are associated with placental and foetal development. Therefore, our subsequent hypotheses were that GHS would: 1) alter placental gene expression of IGF-2 and vascular endothelial growth factor (VEGF); and, 2) affect maternal and foetal circulating endocrine profiles.

Material and Methods Fifteen primiparous pregnant gilts (Large White $\mathrm{x}$ Landrace; $112 \pm 5 \mathrm{~kg}$, mean $\pm \mathrm{SD}$ ) were randomly allocated to either thermoneutral conditions (GTN; constant $28^{\circ} \mathrm{C}, \mathrm{n}=7$ ) or cyclical heat stress conditions (GHS; $8 \mathrm{~h} / \mathrm{d}, 28-33^{\circ} \mathrm{C}, \mathrm{n}=8$ ) between d 40 to 60 of gestation. All pigs were exposed to an artificial $12 \mathrm{~h} / \mathrm{d}$ light regimen and were fed a gestational diet containing $13 \mathrm{MJ} / \mathrm{kg}$ digestible energy (DE) $/ \mathrm{kg}$. On the last day of thermal treatments, maternal blood was obtained via jugular venipuncture for plasma endocrine assays. Following euthanasia of the gilts, foetuses and placentas were harvested from the reproductive tract, and foetal blood was withdrawn via cardiac puncture. Placental IGF-2 and VEGF mRNA abundance were quantified using RT-qPCR and presented as relative normalized expression. Data were analysed using Linear Mixed Models (GenStat $18^{\text {th }}$ ed, VSN International, Hemel Hempstead, UK).

Results Placental IGF-2 mRNA relative expression was up-regulated in GHS placentae (1.00 vs 1.26, $P=0.037)$, while the VEGF mRNA abundance was not significantly different $(1.00$ vs $0.95, P=0.29)$. Heat stressed gilts had lower circulating cortisol and progesterone concentrations $(P=0.05$ and 0.036 , respectively; Table 1), while plasma total IGF1 concentrations remained unchanged $(P=0.14)$. Both foetal plasma thyroid hormone (free T4 and T3) and cortisol concentrations did not differ between GTN and GHS treatments (Table 1).

Table 1. Placental IGF-2 and VEGF mRNA relative expression and circulating endocrine variables of the gilts (maternal) and foetuses (foetal) subjected to gestational thermoneutral treatment (GTN) or gestational heat stress treatment (GHS).

\begin{tabular}{|c|c|c|c|c|c|c|c|c|}
\hline & \multirow{2}{*}{\multicolumn{2}{|c|}{$\begin{array}{l}\text { Placental mRNA } \\
\text { relative expression }\end{array}$}} & \multicolumn{3}{|c|}{ Maternal circulation } & \multicolumn{3}{|c|}{ Foetal circulation } \\
\hline & & & \multirow{2}{*}{$\begin{array}{c}\text { Progesterone } \\
(\mathrm{ng} / \mathrm{ml})\end{array}$} & \multirow{2}{*}{$\begin{array}{l}\text { IGF-1 } \\
(\mathrm{ng} / \mathrm{ml})\end{array}$} & \multirow{2}{*}{$\begin{array}{c}\text { Cortisol } \\
(\mu \mathrm{g} / \mathrm{dl})\end{array}$} & \multirow{2}{*}{$\begin{array}{c}\text { Cortisol } \\
(\mu \mathrm{g} / \mathrm{dl})\end{array}$} & \multirow{2}{*}{$\begin{array}{c}\text { Free T4 } \\
(\mathrm{ng} / \mathrm{dl})\end{array}$} & \multirow{2}{*}{$\begin{array}{r}\text { Free T3 } \\
(\mathrm{pg} / \mathrm{ml}) \\
\end{array}$} \\
\hline & IGF-2 & VEGF & & & & & & \\
\hline GTN & $1.00^{\mathrm{a}}$ & 1.00 & $17.70^{\mathrm{a}}$ & 80.9 & $5.74^{\mathrm{a}}$ & 1.08 & 0.20 & 0.34 \\
\hline GHS & $1.26^{\mathrm{b}}$ & 0.95 & $15.52^{\mathrm{b}}$ & 68.7 & $4.02^{\mathrm{b}}$ & 0.98 & 0.21 & 0.35 \\
\hline SED & 0.127 & 0.074 & 0.908 & 7.72 & 0.806 & 0.144 & 0.017 & 0.072 \\
\hline$P$ value & 0.037 & 0.29 & 0.036 & 0.14 & 0.05 & 0.67 & 0.93 & 0.48 \\
\hline
\end{tabular}

a,b ${ }^{2}$ Means within a column with different superscripts differ significantly $(P<0.05)$; IGF, insulin-like growth factor; VEGF, vascular endothelial growth factor; SED, standard error of the difference of the means.

Conclusion Our hypotheses were supported by the data that heat stress during mid-gestation up-regulates placental IGF2 mRNA relative expression and reduced maternal circulating progesterone and cortisol levels. These data, coupled with morphological enlargement of placental mass and size, possibly suggest adaptive responses of placentas to GHS, which have implications on foetal development. Further work is required to determine the impacts of GHS on placental function.

Acknowledgements Supported in part by Australian Pork Limited (Project number 2017/2213) and Postgraduate Research Scholarship, and the Melbourne Research Scholarship. The authors acknowledge the assistance provided by staff and students from the Animal Production research group, Faculty of Veterinary and Agricultural Sciences, The University of Melbourne.

\section{References}

Bell AW, McBride BW, Slepetis R, Early RJ and Currie WB 1989. Journal of Animal Science. 67, 3289-3299. Zhao W, Liu F, Bell AW, Cottrell JJ, Leury BJ and Dunshea FR 2019. In Book of Abstracts of the $70^{\text {th }}$ Annual Meeting of the European Federation of Animal Science. Wageningen Academic Publishers, the Netherlands. In press 


\section{A comparison of placenta and piglet birth weight of sows mated in summer and autumn}

$\underline{\text { F Liu }}^{1}$, E M Ford ${ }^{1}$, R J Smits ${ }^{1}$, R S Morrison ${ }^{1}$, C J Brewster ${ }^{1}$, D J Henman ${ }^{1}$, W Zhao ${ }^{2}$, B J Leury ${ }^{2}$, J J Cottrell ${ }^{2}$, F R Dunshea $^{2}$ and A W Bell ${ }^{3}$

${ }^{1}$ Rivalea Australia Pty Ltd, Australia, ${ }^{2}$ The University of Melbourne, Australia, ${ }^{3}$ Cornell University, USA

fliu@rivalea.com.au

Application An increased percentage of light piglets were born to sows mated in summer compared to autumn, which may partially explain the greater carcass backfat in finishers observed around October (spring) in Australia.

Introduction The profitability of Australian pork production is driven by meeting strict carcass weight and backfat requirements. Light-born piglets, known to grow with greater adiposity (Poore and Fowden, 2004), may be partially responsible for the peaked carcass backfat thickness of finishers observed around October in Australia - a phenomenon that is not fully explained by carcass weights (Trezona et al., 2004). The hypothesis was that October finisher pigs that are born to sows mated in summer have lower birth weights possibly due to heat stress induced reductions in placental efficiency.

Material and methods Farrowing outcomes of multiparous sows (Large White $\mathrm{x}$ Landrace, Primegro ${ }^{\mathrm{TM}}$ Genetics, Corowa, NSW) that were mated in summer between 15-26 February $(\mathrm{n}=119)$ and autumn between 23 May - 2 June $(\mathrm{n}=118)$ in 2018 were studied. Sow parity was similar between the two mating seasons $(3.7 \pm 1.3$, mean $\pm \mathrm{SD})$. Sows were group housed and their average daily feed allowance was provided at $2.2 \mathrm{~kg} / \mathrm{d}$ using electronic sow feeders. The average daily maximum in-shed temperature was $28.8 \pm 2.6^{\circ} \mathrm{C}$ and $14.2 \pm 3.1^{\circ} \mathrm{C}$ for the first four weeks after summer and autumn matings, respectively. Fifteen focal sows from each mating season were monitored for heat stress signs (respiration rate and rectal temperature), at $1700 \mathrm{~h}$ once per week post-mating. Body weight of sows ( $\mathrm{n}=15$ per season) were measured on the second day after mating. On average, five placentas and five piglets from each focal litter were individually tagged, matched and weighed after birth ( $\mathrm{n}=38$ focal litters per season). Birth weight of live piglets was individually recorded in all litters. Data were analysed by General Linear Model (IBM SPSS Statistics for Windows, v25.0. Armonk, NY).

Results During the first four weeks gestation rectal temperature $\left(38.4^{\circ} \mathrm{C}\right.$ vs $38.1^{\circ} \mathrm{C}$, standard error $\left.(\mathrm{SE})=0.07\right)$ and respiration rate $(25.4$ vs 17.4 breaths/minute, $\mathrm{SE}=0.90)$ were both significantly $(P<0.001)$ greater for sows mated in summer than those mated in autumn, suggesting mild heat stress. The sows mated in summer had lower post-mating body weight $(P<0.05)$ and produced an increased percentage of light-born piglets $(\leq 1.1 \mathrm{~kg}$, Table 1$)$. Reduced litter weight at birth, individual piglet birth weight and placental weight were also observed in the sows mated in summer (all $P<$ 0.05 ), although the number of piglets born alive was similar. The placental efficiency (piglet birth weight:placental weight) was higher $(P=0.054)$ for those sows mated in summer, suggesting an effort to maintain nutrient supply to foetuses.

Table 1. Farrowing outcomes of sows mated in summer vs autumn (estimated marginal means with standard errors)

\begin{tabular}{|c|c|c|c|c|c|c|c|c|c|}
\hline $\begin{array}{l}\text { Total } \\
\text { number } \\
\text { of litters }\end{array}$ & $\begin{array}{c}\text { Sow } \\
\text { weight } \\
\text { post- } \\
\text { mating } \\
(\mathrm{kg}) \\
(\mathrm{n}=30)\end{array}$ & $\begin{array}{c}\text { Number } \\
\text { of born } \\
\text { alive } \\
\text { piglet }\end{array}$ & $\begin{array}{c}\text { Number } \\
\text { of } \\
\text { stillborn } \\
\text { piglet }\end{array}$ & $\begin{array}{c}\text { Litter } \\
\text { birth } \\
\text { weight } \\
\text { (born } \\
\text { alive }) \\
(\mathrm{kg}) \\
(\mathrm{n}=237)\end{array}$ & $\begin{array}{c}\% \text { of } \\
\text { piglets } \\
\leq 1.1 \mathrm{~kg}\end{array}$ & $\begin{array}{c}\text { Piglet } \\
\text { birth } \\
\text { weight } \\
\text { (born } \\
\text { alive }) \\
(\mathrm{kg}) \\
(\mathrm{n}=237)\end{array}$ & $\begin{array}{c}\text { Placenta } \\
\text { weight } \\
(\mathrm{kg})\end{array}$ & $\begin{array}{c}\text { Piglet } \\
\text { weight } \\
\text { for } \\
\text { matched } \\
\text { placenta } \\
(\mathrm{kg}) \\
(\mathrm{n}=76)\end{array}$ & $\begin{array}{c}\text { Piglet: } \\
\text { placenta } \\
\text { weight } \\
\text { ratio }\end{array}$ \\
\hline & 250 & & 1.4 & & & & & & 5.73 \\
\hline & 270 & & & & 15 & & & & \\
\hline SE & 4.1 & 0.29 & 0.18 & 0.3 & 0.015 & 0.021 & 0.012 & 0.038 & 0.28 \\
\hline$P$ value & 0.003 & 0.73 & 0.92 & 0.024 & $<0.001$ & $<0.001$ & 0.002 & 0.040 & 0.054 \\
\hline
\end{tabular}

Conclusion Sows mated in summer had lighter placentas and piglets than those mated in autumn, which may partially explain the greater carcass backfat in progeny observed around October in Australia. Whether the impacts were due to the weight loss of sows from previous summer lactation or the heat stress in early gestation remains to be further explored.

Acknowledgements Supported in part by Australian Pork Limited (Project number 2017/2213) and supported by staff at Research and Innovation Unit and Farming Operations of Rivalea Australia Pty Ltd. We thank Dr Rebecca Athorn (APL) for her professional input in experimental design.

\section{References}

Trezona M, Mullan BP, D'Antuono M, Wilson RH and Williams IH 2004. Australian Journal of Agricultural Research. 55, 273-277.

Poore KR and Fowden AL 2004. The Journal of Physiology. 558, 295-304. 


\section{Birth weight is an early indicator of reproductive development in the gilt}

L M Staveley $^{1,3}$, K Kind ${ }^{1}$, J Kelly ${ }^{2}$ and W H E J van Wettere ${ }^{1}$

${ }^{1}$ The University of Adelaide, Australia, ${ }^{2}$ South Australian Research and Development Institute, Australia, ${ }^{3}$ SunPork Group, Australia

lauren.staveley@sunporkfarms.com.au

Application Exclusion of the lowest birth weight animals from each litter will ensure only the highest fertility gilts will enter the breeding herd at selection, as weight differences at puberty attainment remain relatively small, and do not account for within litter variability.

Introduction The selection of replacement gilts has generally been focused around physical structure and weight prior to and during puberty attainment (weeks 16-21), however it is accepted in many species that the prenatal environment plays an equally important role in reproductive development as the pre-pubertal environment (Mousseau and Fox, 1998). Intrauterine growth restriction is evident at birth but can be less so later in life, as these animals will often show accelerated growth, termed "catch-up growth", which in many species is predictive of later impairment of health and function (De Blasio et al., 2006). As the Australian pig industry continues to select for highly prolific sows there will be an inevitable increase in compromised and lightweight piglets (Campos et al., 2010). There have been a number of studies into the effects of low birth weight (BW) on ovarian developmental measures pre-weaning, however the aim of this study was to investigate the effect of within litter low vs high birth weight on ovarian development at age of selection.

Material and methods This study used the two highest and lowest weight female piglets from 166 litters, identified and weighed at d 1, 21 and 154 of life, however only a subset of 214 animals were analysed as this was part of a larger study. Reproductive tracts were collected post slaughter at d $154 \pm 9$ with both ovaries weighed and antral follicles counted and classified as either small (1-3.99 $\mathrm{mm})$ or large $(\geq 4 \mathrm{~mm})$, only large follicle diameter was measured. Neonatal fractional growth rate (NFGR), which is the measure of "catch-up growth", was calculated as the average daily gain (ADG) from 1 to $21 \mathrm{~d}$ of age and divided by the $\mathrm{d} 1$ weight of the piglet. Linear mixed models with birth weight, litter size, maternal parity, and season farrowed (in lieu of batch) and their interactions as fixed effects, and maternal sow as a random term were run for all variables (IBM SPSS Statistics for Windows, v25.0. Armonk, NY). Weaning weight was calculated as weight at $\mathrm{d} 14$, due to changes in piggery management resulting in early weaning ages during this study.

Results BW significantly affected all measures of weight gain, with high BW animals remaining heavier throughout their life. ADG was increased in these high BW animals, but NFGR was decreased. BW was negatively correlated with small antral follicle counts $(r=-0.12, P<0.05)$ and positively correlated with large antral follicle counts $(r=0.12, P<0.05)$, weaning weight was positively correlated with small antral follicle counts $(r=0.33, P<0.05)$, and slaughter weight was positively correlated with ovarian weight $(r=0.39, P<0.05)$, and average follicle diameter $(r=0.39, P<0.05)$.

Table 1. Mean \pm SEM gilt weight $(\mathrm{kg})$ at 1 and $14 \mathrm{~d}$ of age when categorised by low and high (within litter) birth weight $(\mathrm{BW})$, including average daily gain $(\mathrm{ADG}, \mathrm{kg} / \mathrm{d})$ and neonatal fractional growth rate (NFGR, $\mathrm{kg} / \mathrm{d})$. Ovarian data at slaughter expressed as average ovarian weight (Ov Wt, g), small antral follicle count (AFC $<4 \mathrm{~mm}$ ), large antral follicle count (AFC $\geq 4 \mathrm{~mm}$ ) and average antral follicle diameter $\geq 4 \mathrm{~mm}$ (AFD).

\begin{tabular}{cccccccccc}
\hline & & & & & & AFC & AFC \\
BW & d 1 Wt & d 14 Wt & ADG & NFGR & d 154 Wt & Ov Wt & $<4 \mathrm{~mm}$ & $\geq 4 \mathrm{~mm}$ & AFD \\
\hline Low & $1.31^{\mathrm{a}} \pm 0.0$ & $4.06^{\mathrm{a}} \pm 0.1$ & $0.213^{\mathrm{a}} \pm 0.0$ & $0.163^{\mathrm{a}} \pm 0.0$ & $89.6^{\mathrm{a}} \pm 1.6$ & $3.9 \pm 0.1$ & $87.8 \pm 4.8$ & $8.9 \pm 0.5$ & $4.9 \pm 0.1$ \\
High & $1.80^{\mathrm{b}} \pm 0.0$ & $4.90^{\mathrm{b}} \pm 0.1$ & $0.235^{\mathrm{b}} \pm 0.0$ & $0.132^{\mathrm{b}} \pm 0.0$ & $94.8^{\mathrm{b}} \pm 1.3$ & $4.2 \pm 0.2$ & $79.1 \pm 4$ & $9.8 \pm 0.5$ & $4.9 \pm 0.1$ \\
\hline
\end{tabular}

${ }^{\mathrm{a}, \mathrm{b}}$ Means within a column with different superscripts differ significantly $(P<0.05)$;

Conclusion Low BW gilts remained significantly lighter until slaughter, however with an average difference of $5.2 \pm 2.9$ $\mathrm{kg}$ this is potentially not distinctive enough to enable easy identification at selection. The premise that nutrients are being partitioned away from reproductive development in the low BW animals is supported by the relatively small weight difference at slaughter and the positive correlations between weaning weight and antral follicle counts, slaughter weight and ovarian weight, and slaughter weight and both preovulatory follicle counts and average follicle diameter. The negative correlation between d 1 weight and small follicles can be explained in conjunction with the positive correlation of preovulatory follicles, suggesting that a greater number of the high BW gilts had reached puberty by d 154, hence the greater number of large antral follicles. As such we can conclude that the hypothesis is supported, with the BW of a gilt proving to be an important factor to consider in breeder selection. The exclusion of low BW animals at this timepoint has the potential to reduce the likelihood of potentially inferior animals being selected due to the significant, although relatively small, weight difference at puberty attainment.

\section{References}

Campos PHRF, Silva BAN, Donzele JL, Oliveira RFM and Knol EF 2012. Animal. 6, 797-806.

De Blasio M, Gatford K, Robinson K and Owens J 2006. American Journal of Physiology-Regulatory, Integrative and Comparative Physiology. 292, 2.

Dwyer CM, Stickland NC and Fletcher JM 1994. Journal of Animal Science. 72, 911-917.

Mousseau TA and Fox CW 1998. Oxford University Press. 


\section{What factors recorded before farrowing affect sow's ability to wean piglets?}

$\underline{\text { L Vargovic }}^{1}$, K L Bunter ${ }^{1}$, S Hermesch ${ }^{1}$, R S Morrison ${ }^{2}$ and R Johnston ${ }^{3}$

${ }^{1}$ Animal Genetics and Breeding Unit, a joint venture of NSW Department of Primary Industries and University of New England, University of New England, Australia, ${ }^{2}$ Rivalea Australia Pty Ltd, Australia, ${ }^{3}$ SunPork Group, Australia lvargovi@myune.edu.au

Application Identifying predictors that indicate the ability of a sow to wean more piglets might offer opportunities for improved sow management.

Introduction The number of weaned piglets has a high impact on profitability per sow and also reflects animal welfare. The most critical period for piglets is within first $72 \mathrm{~h}$ after birth, where common reasons for pre-weaning mortality are crushing and starvation. Over the last twenty years, there has been a linear increase in the number of live born piglets, while trends for pre-weaning mortality fluctuate (Baxter and Edwards, 2018). This study tested the hypothesis that close observation of sows upon transfer to the farrowing shed for possible health indicators might identify factors which have an impact on the number of piglets weaned by individual sows.

Material and methods Data were available for 1088 sows recorded at two nucleus farms located in NSW, Australia. Sows were recorded for a range of attributes upon transfer to the farrowing shed, including: locomotion score, with lameness increasing from $0-2$; dirtiness of the udder, scored as clean $=0$ or dirty $=1$; teat access, scored from $1-3$, reflecting increasing interference of crate to teat access; the extent of fight lesions: scored from 0 (no lesions) to 2, in increments of 5 lesions per score; body condition score assessed by caliper increments (Knauer and Baitinger, 2015), Score 0: <11; 1: [11-12]; 2: [13-14]; 3: [15-16] and 4: > 16; and count of injured teats, scored from 0 (no teats injured) to 3 (3+ injured teats). The trait analysed was the number of weaned piglets (NWEAN). Sows which failed to wean any piglets, due to their death or removal, received trait values of NWEAN=0. A generalised linear model, assuming a Poisson distribution, was applied to NWEAN using the GLM procedure in R 3.5.1 (R Foundation, Vienna, Austria). The basic model accounted for breed group (maternal vs terminal) nested within farm, parity group (gilts: 1 vs sows: 2) and additional terms noted above in a multi-variate analysis. Cross-fostering was not recorded and was not accounted for. Means were compared using Tukey's test, with model terms approaching significance $(P<0.10)$ also shown, to better illustrate the impact of class effects with low incidence (e.g. teat injuries).

Results Poor locomotion score and restricted teat access significantly $(P<0.001)$ reduced the number of weaned piglets (Table 1). Sows with fight lesions score $\geq 1$ tended to wean more piglets than sows with no lesions $(P=0.053)$. Very thin (caliper $=0$ ) and very fat (caliper $=4$ ) sows also tended to wean less piglets than sows in the targeted body condition (caliper=1-3), as did sows with increasing pre-farrowing teat injuries scores $(P=0.051)$.

Table 1. Least square means for the number of weaned piglets, Breed group:Farm (levels 1:2 maternal breeds for farms $\mathrm{A}$ and $\mathrm{B}, 3: 4$ terminal breeds)

\begin{tabular}{|c|c|c|c|c|c|c|}
\hline \multirow[b]{2}{*}{ Predictor } & \multicolumn{5}{|c|}{ NWEAN for each level of predictor } & \multirow[b]{2}{*}{$P$ value } \\
\hline & 0 & 1 & 2 & 3 & 4 & \\
\hline Breed group:Farm & & $9.80^{\mathrm{a}} \pm 0.15$ & $9.11^{\mathrm{b}} \pm 0.16$ & $9.16^{\mathrm{b}} \pm 0.34$ & $8.59^{\mathrm{b}} \pm 0.27$ & $<0.001$ \\
\hline Parity & & $9.06^{\mathrm{a}} \pm 0.18$ & $9.46^{\mathrm{a}} \pm 0.12$ & & & 0.079 \\
\hline Locomotion score & $9.45^{\mathrm{a}} \pm 0.10$ & $8.78^{\mathrm{ab}} \pm 0.32$ & $7.52^{\mathrm{b}} \pm 0.48$ & & & $<0.001$ \\
\hline Dirty udder & $9.29^{\mathrm{a}} \pm 0.10$ & $10.4^{\mathrm{b}} \pm 0.51$ & & & & 0.022 \\
\hline Teat access & & $9.52^{\mathrm{a}} \pm 0.14$ & $9.58^{\mathrm{b}} \pm 0.19$ & $8.57^{\mathrm{b}} \pm 0.22$ & & $<0.001$ \\
\hline Fight lesions & $8.92^{\mathrm{a}} \pm 0.19$ & $9.46^{\mathrm{a}} \pm 0.16$ & $9.52^{\mathrm{a}} \pm 0.17$ & & & 0.053 \\
\hline Caliper score & $8.48^{\mathrm{a}} \pm 0.35$ & $9.49^{\mathrm{a}} \pm 0.24$ & $9.37^{\mathrm{a}} \pm 0.17$ & $9.57^{\mathrm{a}} \pm 0.19$ & $9.17^{\mathrm{a}} \pm 0.22$ & 0.074 \\
\hline Teat injuries & $9.49^{\mathrm{a}} \pm 0.12$ & $9.34^{\mathrm{a}} \pm 0.19$ & $9.04^{\mathrm{a}} \pm 0.27$ & $8.50^{\mathrm{a}} \pm 0.35$ & & 0.051 \\
\hline
\end{tabular}

${ }^{\mathrm{a}, \mathrm{b}}$ Means in a row with different superscripts differ significantly, $P<0.05$ (Tukey method).

Conclusion Poor locomotion, restricted teat access, teat injuries and low caliper scores pre-farrowing reduced the number of weaned piglets. Poor locomotion has a negative impact on sow's ability to prevent piglet crushing and can influence the sow's ability to eat and drink (Edwards and Baxter, 2015). Avoidance or management of these conditions may improve performance outcomes.

Acknowledgements Supported in part by Pork CRC Limited (Project 2A-116) and the UNE via International Postgraduate Research Award (UNE IPRA).

\section{References}

Baxter EM and Edwards SA 2018. In Advances in Pig Welfare (Ed: Špinka M), Woodhead Publishing, UK. pp. 73-100. Edwards DB and Baxter EM 2015. In The gestating and lactating sow (Ed: Farmer C), Wageningen Academic Publisher, the Netherlands. pp. 253-269.

Knauer MT and Baitinger DJ 2015. Applied Engineering in Agriculture. 31, 175-178. 


\section{Increasing essential amino acids in late gestation improves birth weight in multiparous sows} D J Cadogan $^{1}$, I Hill ${ }^{2}$, H van Sliedregt ${ }^{3}$ and R O Ball ${ }^{4}$

${ }^{1}$ Feedworks, Australia, ${ }^{2}$ Bailey Creek Pork, Australia, ${ }^{3}$ Riverbend, Australia, ${ }^{4}$ University of Alberta, Canada david.cadogan@feedworks.com.au

Application Novel nutritional strategy to increase birth weight and possibly subsequent reproductive performance.

Introduction Previously, most authorities and genetic companies have recommended a constant nutrient and energy supply during gestation and many operations still abide by this strategy. However, birth weight is falling significantly as modern prolific sows have higher litter numbers (Wilson, 2014). In an attempt to arrest this decline in birth weight, increased dietary energy during late gestation was proposed. Yet several experiments have shown that increasing energy during late gestation had no significant effect on birth weight and had a negative effect on sow feed intake during lactation (Ball et al., 2008). Models developed recently (eg NRC, 2012) have attempted to predict the essential amino acid and energy requirements of the gestating sow in order to better match the dietary specification to the requirements of the sow (Ball and Moehn, 2013). This study hypothesised that supporting the rapidly increasing essential amino acid levels as reported by Ball and Moehn (2013) in late gestation will increase birth weight and potentially improve subsequent reproductive performance.

Material and methods Three hundred and thirty-four multiparous (average parity $3.6 \pm 0.36$ ) PIC sows with an average of $261 \pm 16.3 \mathrm{~kg}$ live weight were housed in a dynamic gestation pen after mating and randomly assigned to one of two diet regimes. Diet regimes were produced by blending two feeds through an electronic sow feeder (ESF). Two $13.5 \mathrm{MJ} / \mathrm{kg}$ digestible energy (DE) gestation diets were formulated to two essential amino acid levels $(0.45$ and $0.55 \mathrm{~g}$ standard ileal digestible (SID) lysine/MJ DE), with the control diet blended by the ESF to produce a SID lysine of $0.48 \mathrm{~g}$ per MJ DE as per PIC nutrient recommended ideal essential amino ratios and offered at $2.2 \mathrm{~kg} / \mathrm{d}$ per sow. The treatment diets were blended by the ESF to achieve essential amino acids levels predicted by Ball and Moehn (2013). For example, the dietary lysine requirement was calculated by regression analysis to be $49 \mathrm{mg} / \mathrm{kg}^{0.75}$. The difference in the treatment diet was the rapid daily, incremental increase in digestible lysine, threonine, isoleucine and tryptophan from d 85 of gestation to parturition. There was no difference in diet specifications between parities due to the limited number of some parities during the study. The sows were weighed at movement to the farrowing shed at $\mathrm{d} 110$ of pregnancy and both treatment groups were offered the same $14 \mathrm{MJ} / \mathrm{kg}$ DE lactation diet at $3 \mathrm{~kg} / \mathrm{d}$ until farrowing. Once the sows farrowed, the number of born alive, still born, individual birth and still born weights were recorded. Birth weights, sow weight gain (SWG) and number born were analysed using a general linear model in JMP software (SAS Institute Inc., Cary, NC, USA).

Results Increasing essential amino acid levels in late gestation significantly increased gestational weight gain of sows by $8.8 \%$. There was no effect on the number total born or born alive, however, the higher essential amino acid diets significantly improved individual live birth and total birth weight by $47 \mathrm{~g}$ and $50 \mathrm{~g}$, respectively (Table 1). The higher birth weights resulted in a significantly greater total litter birth weight. Subsequent reproductive performance results were not available at time of submission.

Table 1. The effect of matching daily essential amino acids requirement in gestation on SWG, birth and total litter

\begin{tabular}{lcccccc}
\hline & $\begin{array}{c}\text { WWG } \\
(\mathrm{kg})\end{array}$ & Total born & Born alive & $\begin{array}{c}\text { TBW } \\
(\mathrm{g})\end{array}$ & $\begin{array}{c}\text { ABW } \\
(\mathrm{g})\end{array}$ & $\begin{array}{c}\text { TLW } \\
(\mathrm{kg})\end{array}$ \\
\hline Control & $42.2^{\mathrm{a}}$ & 12.9 & 11.2 & $1266^{\mathrm{a}}$ & $1282^{\mathrm{a}}$ & $13.97^{\mathrm{a}}$ \\
Treatment & $47.8^{\mathrm{b}}$ & 13.3 & 11.6 & $1316^{\mathrm{b}}$ & $1329^{\mathrm{b}}$ & $15.22^{\mathrm{b}}$ \\
SE & 1.62 & 0.24 & 0.23 & 16.3 & 17.1 & 0.29 \\
$P$ value & 0.004 & 0.312 & 0.144 & 0.034 & 0.045 & 0.003 \\
\hline
\end{tabular}

a,b Means within a column with different superscripts differ significantly $(P<0.05)$. SWG sow weight gain, TBW Total birth weight, ABW alive birth weight, TLW total litter weight

Conclusion Increasing essential amino acid levels in late gestation as predicted by Ball and Moehn (2013) increased birth weight by $3.5 \%$ and supports the theory that essential amino acids, not energy, in late gestation is perhaps the bigger driver of birth weights in the modern prolific sow.

\section{References}

Ball RO, Samuel RS and Moehn S 2008. Advances in Pig Production. 19, 223-236.

Ball RO and Moehn S 2013. Manipulating Pig Production. 14, 151-169.

NRC 2012. Nutrient Requirements of Swine: $11^{\text {th }}$ Revised Edition. The National Academies Press, Washington DC, USA. Wilson S (2014). https://pork.ahdb.org.uk/media/72741 


\section{Evaluation of sow and piglet characteristics related to stillborn rate and pre-weaning survival in high producing sows}

K M Gourley ${ }^{1}$, H I Calderon ${ }^{1}$, A J Swanson ${ }^{1}$, J C Woodworth ${ }^{1}$, J M DeRouchey ${ }^{1}$, M D Tokach ${ }^{1}$, R D Goodband ${ }^{1}$ and S S Dritz ${ }^{1}$

${ }^{1}$ Kansas State University, USA

kgourley@k-state.edu

Application In sows with large litter sizes, short parturition time reduces stillborn rate and tends to improve survival of live born pigs to weaning. Increased birth weights, colostrum intake, and teat pig ratio contribute to improved piglet survival, whereas birth order does not affect live born survival. These results suggest that reduction in farrowing duration may help increase weaned pigs per litter.

Introduction As litter size increases, piglet survival becomes a challenge due to factors including low birth weight, competition for teats, colostrum intake, and increased farrowing duration. The objective of this study was to determine the effects of piglet birthweight, birth order, farrowing duration, teat to pig ratio, and colostrum intake on piglet survival to weaning in high producing sows.

Material and methods A total of 6939 piglets from 472 litters (DNA 600 sire x F1 Landrace x Large White dam) were used. At birth, all pigs (including stillborn) were weighed, tagged, and birth time recorded. At $24 \mathrm{~h}$ after birth of the first pig in the litter, piglets were individually reweighed to calculate colostrum intake. All piglet mortalities were weighed with the date recorded. At weaning (d $20 \pm 3$ ), all piglets were individually weighed. Data for each response criteria was divided into quartiles to create categorical responses. Categorical variables include; birth order, birth weight, $24 \mathrm{~h}$ piglet weight, colostrum intake, sow farrowing duration, teat to pig ratio, and total born. Piglet data was analysed using Pearson's chi-squared test for relationships between categorical variables and survival to d 3, d 7, and weaning. Associations were considered significantly at $P<0.05$, and a tendency between $0.05 \leq P<0.10$.

Results An increased percentage of stillborn pigs was observed as litter size increased. Birth order (first to last) did not influence survival of live born pigs to weaning. However, the odds of being a stillborn pig increased as pigs were born later in the birth order. Pigs in the lowest quartile for weight at birth $(<1205 \mathrm{~g})$ or at $24 \mathrm{~h}(<1300 \mathrm{~g})$ had a greater mortality percentage at weaning compared to pigs in heavier weight categories. Percentage of stillborn pigs increased, and piglet survival to weaning tended to decrease for pigs in the highest farrowing duration quartile ( $>308 \mathrm{~min})$. Pigs in the lowest colostrum intake quartile $(<336 \mathrm{~g})$ or lowest teat to pig ratio quartile $(<0.94$ teat:pig) had reduced survival to weaning compared to pigs with more colostrum intake or an increased teat to pig ratio, respectively.

Table 1. Percentage of pigs weaned and stillborn within response category quartiles

\begin{tabular}{lccccc}
\hline \multicolumn{7}{c}{} & \multicolumn{3}{c}{$\chi^{2} P$ value } \\
\hline Total Born, $\mathrm{n}$ & $<13$ & $13-15$ & $16-17$ & $>17$ & \\
Stillborn, \% & 4.3 & 5.4 & 6.8 & 9.9 & $<0.001$ \\
& & & & & \\
Birth order ranking & $<25 \%$ & $25-50 \%$ & $51-75 \%$ & $>75 \%$ & \\
Weaned, \% & 93.3 & 94.0 & 93.9 & 93.9 & 0.215 \\
Stillborn, \% & 1.5 & 3.0 & 6.3 & 17.2 & $<0.001$ \\
& & & & & \\
Birth weight, g & $<1205$ & $1205-1440$ & $1441-1670$ & $>1671$ & \\
Weaned, \% & 85.1 & 94.6 & 95.8 & 97.8 & $<0.001$ \\
Stillborn, \% & 10.8 & 6.7 & 5.0 & 5.1 & $<0.001$ \\
& & & & & \\
Farrowing duration, min & $<162$ & $162-225$ & $226-308$ & $>308$ & 0.085 \\
Weaned, \% & 94.5 & 94.5 & 93.4 & 92.4 & 8.9 \\
Stillborn, \% & 4.5 & 6.0 & 6.8 & & $<01$ \\
& & & & & \\
Teat to pig ratio & $<0.94$ & $0.95-1.07$ & $1.08-1.25$ & $>1.25$ & \\
Weaned, \% & 88.7 & 93.8 & 96.6 & 96.2 & $<0.001$ \\
\hline
\end{tabular}

Conclusion This study quantifies the relationship between litter variables, stillborn, and piglet survival to weaning in sows with large litter sizes. Piglet birth order did not appear to influence live born piglet survival as hypothesised; however, pigs born at the end of the parturition had increased odds of being stillborn. Any pig that is born from a litter with a long farrowing duration tended to have a reduced survival chance regardless of birth order. Therefore, future research should be directed towards nutritional or management strategies to reduce farrowing duration.

Acknowledgements Appreciation is expressed to the Minnesota Pork Board for funding, Christensen Farms for use of animals and facilities, and Kansas State University graduate students and professors for assistance with data collection. 


\section{Anti-inflammatory administration to sows prior to farrowing does not improve piglet survival or growth}

K J Plush ${ }^{1}$, J R Pluske ${ }^{2}$, D S Lines ${ }^{1}$, C R Ralph ${ }^{3}$ and R N Kirkwood ${ }^{4}$

${ }^{1}$ SunPork Group, Australia, ${ }^{2}$ Murdoch University, Australia, ${ }^{3}$ Agersens, Australia, ${ }^{4}$ The University of Adelaide, Australia kate.plush@sunporkfarms.com.au

Application Neither non-steroidal (meloxicam) or steroidal (dexamethasone) anti-inflammatory compounds are suitable agents to promote piglet survival and growth when administered before farrowing.

Introduction Parturition is associated with both pain and inflammation. Pain is likely to cause sow restlessness at farrowing, which will put piglets at risk from sow overlay. Inflammation is linked to reduced milk output in dairy cows (Farney et al., 2013), and both dexamethasone and meloxicam are anti-inflammatory agents (Maślanka et al., 2013). The aim of this experiment was to determine if steroidal and non-steroidal anti-inflammatory agents given to sows before farrowing would reduce pain, restlessness and inflammation, thereby increasing piglet survival and growth.

Material and methods At d 114 of gestation, multiparous sows were randomly allocated to one of the following treatments; Control $(\mathrm{n}=40), 10 \mathrm{~mL}$ saline; SAID $(\mathrm{n}=53), 1 \mathrm{~mL} / 20 \mathrm{~kg}$ dexamethasone $2 \mathrm{mg} / \mathrm{mL}$; and NSAID ( $\mathrm{n}=55), 1$ $\mathrm{mL} / 50 \mathrm{~kg}$ meloxicam $20 \mathrm{mg} / \mathrm{mL}$, all via intramuscular injection. Treatments were applied again on $\mathrm{d} 116$ if farrowing had not occurred. Piglets born alive (BA), born dead (BD) and postnatal piglet deaths were recorded. Piglet colostrum intake was estimated by percent plasma protein via Brix refractometry, and sow rectal temperature taken to indicate incidence of mastitis-metritis-agalactia (MMA). Litters were fostered within treatment and weighed on d 1 and d 21 of lactation. The feed delivered to sows was used to estimate average daily feed intake. An appropriate linear mixed model was applied to the data based on distribution with the fixed effects of room, parity group, treatment, and the interaction between parity and treatment (IBM SPSS Statistics for Windows, v25.0. Armonk, NY).

Results Average gestation length was $116.5 \pm 0.2 \mathrm{~d}$ and was unaffected by treatment. There was no treatment effect on piglets BA or BD from parity 2 to 4 sows, but in parity 5 and older, NSAID administration reduced piglets BA and increased piglets BD $(P<0.05)$. Incidence of MMA was unaffected by treatment, but a tendency for reduced piglet Brix protein in NSAID and SAID litters was identified. Treatment effects were not observed for BA mortality, or litter weight at $\mathrm{d} 21$. Average feed intake was improved by both NSAID and SAID treatments $(P=0.001)$.

Table 1. The mean \pm SEM lactation performance of sows administered saline (Control, $10 \mathrm{~mL}$ ), meloxicam (NSAID, 1 $\mathrm{mL} / 50 \mathrm{~kg},(20 \mathrm{mg} / \mathrm{mL}))$ or dexamethasone (SAID, $1 \mathrm{~mL} / 20 \mathrm{~kg},(2 \mathrm{mg} / \mathrm{mL}))$ prior to farrowing.

\begin{tabular}{lcccc}
\hline & Control & NSAID & SAID & $P$ value \\
\hline Gestation length (days) & $116.5 \pm 0.3$ & $116.4 \pm 0.2$ & $116.8 \pm 0.2$ & 0.225 \\
Born alive & $12.4 \pm 0.9$ & $10.7 \pm 1.2$ & $11.9 \pm 0.8$ & 0.525 \\
Born dead & $0.78 \pm 0.17$ & $0.98 \pm 0.14$ & $1.04 \pm 0.16$ & 0.536 \\
Incidence of MMA (\%) & $20(10-38)$ & $6(2-18)$ & $9(3-24)$ & 0.137 \\
Piglet Brix protein (\%) & $6.8 \pm 0.4$ & $5.6 \pm 0.4$ & $5.8 \pm 0.4$ & 0.097 \\
Piglet mortality & $2.2 \pm 0.3$ & $2.4 \pm 0.2$ & $2.0 \pm 0.2$ & 0.418 \\
Litter size d 21 & $9.5 \pm 0.3$ & $9.5 \pm 0.2$ & $9.3 \pm 0.3$ & 0.808 \\
Litter weight d 21 (kg) & $59.7 \pm 2.3$ & $60.5 \pm 1.8$ & $59.4 \pm 1.9$ & 0.921 \\
Average feed intake/d (kg) & $7.1^{\mathrm{a}} \pm 0.1$ & $7.4^{\mathrm{b}} \pm 0.1$ & $7.5^{\mathrm{b}} \pm 0.1$ & 0.002 \\
\hline
\end{tabular}

${ }^{\mathrm{a}, \mathrm{b}}$ Means within a row with different superscripts differ significantly $(P<0.05)$; SEM, standard error of the mean; $* \mathrm{CI}$ rather than SEM given for binary data.

Conclusion The increase in piglets born dead to NSAID-treated sows could be driven by the combined actions of inhibited prostaglandin synthesis reducing uterine contractility, as well as potentially poorer foetal heart function. Piglets from SAID-treated sows may be buffered from the latter via the rise in corticosteroid-binding globulin prior to parturition. Whilst there was an increase in sow feed intake for NSAID-treated sows, this did not translate to improvements in piglet growth. Anti-inflammatory compound administration to sows before farrowing is not recommended.

Acknowledgements Supported in part by Pork CRC Limited. Jena Alexopoulos, Dannielle Glencorse and Jemma Seyfang are thanked for technical assistance.

\section{References}

Farney J, Mamedova L, Coetzee J, Minton J, Hollis L and Bradford B 2013. Journal of Dairy Science. 96, 7709-18. Maślanka T, Jaroszewski J, Markiewicz W, Jasiecka A, Ziółkowski H and Jędrzkiewicz D 2013. Research in Veterinary Science. 94, 662- 674. 


\section{Dextrose supplementation in the wean to mate period increases litter size and maintains piglet viability}

D Glencorse $^{1}$, J G Alexopoulos ${ }^{1}, \mathrm{~S}$ M Tritton ${ }^{1}$, D N D’Souza ${ }^{1}$ and K J Plush ${ }^{1}$

${ }^{1}$ SunPork Group, Australia

dannielle.glencorse@sunporkfarms.com.au

Application The inclusion of dextrose in sow wean-to-mate diets enhances total piglets born whilst maintaining piglet viability and is a suitable dietary addition to increase litter size.

Introduction Seasonal changes negatively impact sow performance, with the most substantial effect being a reduction in conception and farrowing rates (De Rensis et al., 2017). It has been proposed that reduced luteinising hormone (LH) concentrations are a potential mechanism to explain seasonal infertility (De Rensis et al., 2017). Campos et al. (2012) suggested that lower levels of LH results in a decrease in the population of large follicles, leading to an abundance of small follicles with minimal LH receptors. If LH secretion is reduced in summer months, ovulation from smaller follicles may be more frequent resulting in more variable oocyte quality (Wetteman and Bazer, 1985). If the follicle population is more uniform, oocyte quality will be enhanced resulting in reduced litter heterogeneity. Bolstering follicle growth through the provision of dextrose in the sow diet during the insemination period has been shown to reduce piglet birth-weight variation (Van den Brand et al., 2005). We hypothesised that piglet birth weight variation would be increased in sows bred during summer, and that dextrose supplementation would ameliorate this manifestation of seasonal infertility.

Material and methods Sows (Parity 2-5) were fed $3.5 \mathrm{~kg}$ of one of two diets (control, standard diet; dextrose, control + $5 \%$ dextrose) from weaning until insemination during summer (March; $\mathrm{N}=290$ ) and winter (August; N=296). At the subsequent farrowing, sow litter performance was recorded including farrowing rate, total number of piglets born, piglets born alive and piglets born dead. All piglets born were weighed individually. Data were analysed using the appropriate linear mixed model for observed distribution (IBM SPSS Statistics for Windows, v25.0. Armonk, NY).

Results The farrowing rate was affected by season with an increase from $64 \%$ in summer to $89 \%$ in winter $(P<0.001)$. Inclusion of dextrose in the wean-to-mate diet of sows caused an increase in the total number of piglets born from 13.6 in control sows to 14.6 in treatment sows $(P=0.025)$. The number of piglets born alive increased from 12.3 in control sows to 13.7 in treatment sows $(P=0.008)$. The increased litter size was not associated with an increase in individual piglet weight variation $(P>0.05)$.

Table 1: Sow farrowing performance of control and dextrose supplemented sows by season. Data are presented as

\begin{tabular}{|c|c|c|c|c|c|c|c|}
\hline \multicolumn{8}{|c|}{ Summer } \\
\hline & Control & Dextrose & Control & Dextrose & Season & Treatment & $\mathrm{S} \times \mathrm{T}$ \\
\hline $\mathrm{N}$ & 148 & 142 & 145 & 151 & & & \\
\hline Farrowing rate $(\%)$ & 56.9 & 69.7 & 88.1 & 89.6 & $<0.001$ & NS & NS \\
\hline Total born & $13.6 \pm 0.4$ & $14.1 \pm 0.4$ & $13.7 \pm 0.4$ & $14.8 \pm 0.4$ & NS & 0.025 & NS \\
\hline Born alive & $12.3 \pm 0.4$ & $12.7 \pm 0.4$ & $12.3 \pm 0.4$ & $13.9 \pm 0.3$ & NS & 0.008 & NS \\
\hline Stillborn & $0.9 \pm 0.1$ & $1.0 \pm 0.1$ & $0.9 \pm 0.1$ & $0.7 \pm 0.1$ & NS & NS & NS \\
\hline Piglet weight (kg) & $1.43 \pm 0.03$ & $1.40 \pm 0.03$ & $1.43 \pm 0.03$ & $1.39 \pm 0.03$ & NS & NS & NS \\
\hline $\mathrm{CV}^{1}$ weight $(\mathrm{kg})$ & $16.9 \pm 0.6$ & $16.4 \pm 0.5$ & $15.6 \pm 0.5$ & $15.9 \pm 0.5$ & NS & NS & NS \\
\hline Piglets $<1.1 \mathrm{~kg}(\%)^{2}$ & 20.8 & 25.8 & 18.5 & 24.3 & NS & NS & NS \\
\hline Pigs weaned & $9.0 \pm 0.3$ & $9.3 \pm 0.2$ & $9.7 \pm 0.2$ & $9.7 \pm 0.2$ & NS & NS & NS \\
\hline
\end{tabular}

${ }^{1}$ Coefficient of variation; ${ }^{2}$ Back-transformed data

Conclusion The inclusion of dextrose in a wean-to-mate sow diet increased subsequent litter size by one piglet without compromising litter homogeneity. These results contradict van den Brand et al. (2005) who identified no change in litter size with a significant reduction in birth weight uniformity, possibly due to the absence of there being a season analysis. The current study indicates that season has a substantial effect on farrowing performance.

Acknowledgements Supported in part by Australian Pork Limited (Project number 2017/2226).

\section{References}

Campos PHRF, Silva BAN, Donzele JL, Oliveira RFM and Knol EF 2012. Animal. 6, 797-806.

De Rensis F, Ziecik AJ and Kirkwood RN 2017. Theriogenology. 96, 111-117.

van den Brand H, Soede NM and Kemp B 2005. Animal Reproduction Science. 91, 353-358.

Wettemann RP and Bazer FW 1985. Journal of Reproduction Fertility Supplement. 33, 199-208. 


\title{
Dietary 1,3-Butanediol increases weight and decreases small for age progeny at birth
} D A Wijesiriwardana ${ }^{1}$, J R Craig ${ }^{2,3}, \mathrm{~J} \mathrm{R} \mathrm{Pluske}^{3}$, J J Cottrell ${ }^{1}$ and F R Dunshea ${ }^{1}$

${ }^{1}$ The University of Melbourne, Australia, ${ }^{2}$ Rivalea Australia Pty Ltd, Australia, ${ }^{3}$ Murdoch University, Australia dwij@student.unimelb.edu.au

\begin{abstract}
Application Supplementing gestation diets with 1,3-Butanediol can increase birth and total litter weights and reduce the percentage of light-for-age gilt progeny and sow progeny by providing an alternative source of energy in the neonatal period. This can lead to increased vigour and milk-seeking behaviour resulting in improved growth performance, reduced pre-weaning mortality, more pigs reaching market and increased profits for the farmer.
\end{abstract}

Introduction Gilt progeny (GP) are born and weaned lighter and have poorer whole-of-life performance than sow progeny (SP, Craig et al., 2017). Low birth weight (LBW) in piglets and reduced vigour are strongly linked and are often the main cause of pre-weaning mortality (Herpin et al., 2002). Glycogen stores provide a primary source of energy within the first 16 hours of life with reduced amounts of these stores found in LBW piglets (Theil et al., 2011). Similar to glucose, ketones are able to pass transplacentally from mother to foetus. Gestation diets supplemented with exogenous ketones offer a novel source of energy to the piglet and may spare metabolism of glycogen stores deposited in utero (Spence, 1985). The aim of this study was to evaluate the use of 1,3-Butanediol (BD), a ketogenic compound, on piglet growth and survival when fed to both primiparous and multiparous sows in late gestation. The hypothesis was that BD would increase birth weights, have fewer light-for-age piglets and decrease pre-weaning mortality in GP.

Material and methods The experiment was a $2 \times 2$ factorial design (parity $\mathrm{x}$ diet) with a total of 77 gilts (parity 1 ) and 74 multiparous sows (parities 2, 3 and 4; Large White $x$ Landrace, Primegro ${ }^{\mathrm{TM}}$ Genetics, Corowa, NSW) being used across a total of five replicates. Diets were randomly allotted and commenced from d 90 where gilts and sows were fed a common gestation diet with the experimental diets containing 4\% BD (1,3-Butylene Glycol, Consolidated Chemical Co, Dandenong South, Australia) substituted for tallow and balanced with wheat. Diets were balanced for energy and protein. Plasma samples were taken at d 110 and analysed for circulating ketones bodies ( $\beta$-OHB). Piglets from each litter were weighed at birth $(n=1822)$ and again at $21 \mathrm{~d}$ of age $(\mathrm{n}=1518)$. Weights were analysed using restricted maximum likelihood (REML, GenStat 16th ed, VSN International, Hemel Hempstead, UK). Pre-weaning mortalities in piglets were analysed using Pearson's Chi-Squared $\left(\chi^{2}\right)$ test, with a value of $P<0.05$ indicating statistical significance and a value of $P<0.10$ was considered a statistical trend.

Results Increased levels of $\beta$-OHB were found in both gilts and sows administered the BD $\operatorname{diet}(0.43 v s 0.52 \mu \mathrm{M}, P=$ 0.006). Individual piglet weights tended to be heavier at birth for progeny born to dams fed $\mathrm{BD}(P=0.085)$ and had a tendency towards heavier total litter weights $(P=0.078)$. At d 21, BD tended to reduce individual piglet weights $(P=$ $0.064)$ with these effects not extending to total litter weights $(P=0.83)$. At $\mathrm{d} 21$, a tendency towards increased litter weights in GP born to the BD diet was observed while reduced total litter weights were observed in SP born to the GP $\operatorname{diet}(P=0.10)$. Gilt progeny were lighter than SP at both time points $(P<0.001)$. Butanediol decreased the percentage of progeny that were light-for-age $(<1.1 \mathrm{~kg})$ at birth $(P=0.034)$. There was no effect of BD on mortality rate $(P=0.64)$.

Table 1. Effects of parity (P) and diet (D) on individual piglet and litter weights in response to feeding BD.

\begin{tabular}{ccccccccc}
\hline & \multicolumn{3}{c}{ Gilt } & \multicolumn{3}{c}{ Sow } & \multicolumn{3}{c}{$P$ value } \\
Weights (kg) & Control & BD & Control & BD & SED & Parity & Diet & P x D \\
\hline Birth (n) & 428 & 468 & 455 & 471 & & & & \\
Individual & 1.34 & 1.39 & 1.51 & 1.56 & 0.02 & $<0.001$ & 0.085 & 0.98 \\
Total litter & 15.5 & 17.1 & 18.5 & 18.7 & 0.69 & $<0.001$ & 0.078 & 0.15 \\
d 21 (n) & 327 & 385 & 395 & 411 & & & & \\
Individual & 5.02 & 4.98 & 6.18 & 6.03 & 0.01 & $<0.001$ & 0.064 & 0.43 \\
Total litter & 47.1 & 50.6 & 66.4 & 62.8 & 3.10 & $<0.001$ & 0.83 & 0.10 \\
\hline
\end{tabular}

Conclusion Birth weights and litter birth weights had a tendency to be increased by the BD diet while also reducing the percentage of light-for-age piglets at birth. Mortality rates were unaffected by the BD diet but were higher in GP compared to SP. Sow progeny continuously outperformed gilt progeny indicating a strong parity effect was still evident throughout the study.

Acknowledgements Supported in part by Australian Pork Limited and the Research and Innovation team at Rivalea Australia Pty Ltd.

\section{References}

Craig JR, Collins CL, Bunter KL, Cottrell JJ, Dunshea FR and Pluske JR 2017. Journal of Animal Science. 95, 49044916.

Herpin P, Damon M and Le Dividich J 2002. Livestock Production Science. 78, 25-45.

Spence CA 1985. Journal of Animal Science. 60, 1280-1287.

Theil PK, Cordero G, Henckel P, Puggard L, Oksjberg N and Sørensen MT 2011. Journal of Animal Science. 89, 18051816. 


\section{Supplementing guanidinoacetic acid in the gestating and lactating sow diet improves lactation feed intake and body condition of sows at weaning}

J R Craig ${ }^{1}$, K S O’Halloran ${ }^{1}$, S L Beer ${ }^{1}$, F Liu $^{1}$, M Rademacher-Heilshorn ${ }^{2}$, S H M Ramos ${ }^{3}$ and D J Henman ${ }^{1}$

${ }^{1}$ Rivalea Australia Pty Ltd, Australia, ${ }^{2}$ Evonik Nutrition \& Care GmbH, Germany, ${ }^{3}$ Evonik SEA Pte Ltd, Singapore jcraig@rivalea.com.au

Application Guanidinoacetic acid can be fed to multiparous sows from day 70 of gestation until weaning to improve sow feed intake and body condition in lactation.

Introduction Guanidinoacetic acid (GAA) is an amino acid derivative and natural precursor of creatine. Creatine is of central importance to energy metabolism, particularly for muscle cells, and lactating sows may be deficient in creatine due to a high requirement for milk production (Brosnan et al., 2009). In high producing animals, de novo synthesis of creatine may be limiting and therefore nutritional supplementation of GAA may result in improved average daily gain (ADG) and feed conversion ratio (FCR) in growing pigs and improved foetal growth in gestating sows. It was therefore hypothesised that supplementation of GAA in the late gestation and lactation diets of sows would improve their lactation feed intake and body condition at weaning, and increase the pre-weaning performance of their litter.

Material and methods A total of 196 multiparous sows (parities 1 to 9 at time of allocation; Large White x Landrace, Primegro $^{\mathrm{TM}}$ Genetics, Corowa, NSW) were selected over 4 time replicates, at d 70 of gestation and were randomly allocated to one of two diets based on parity: a control dietary regime based on the current commercial diet (parity 3.0 \pm 0.2 , mean $\pm \mathrm{SE}$ ); or the current commercial diet supplemented with $1 \mathrm{~g} / \mathrm{kg}$ of GAA (parity $3.4 \pm 0.2$ ). Diets were fed from d 70 of gestation until weaning, changing from a commercial gestation to a commercial lactation diet at entry to the farrowing house (d $109 \pm 0.1$ of gestation). Sows were weighed when they entered the farrowing shed and again at weaning. Total litter weight and number of piglets were recorded at fostering and again at d 20 of lactation. Minimal fostering within treatment was carried out $(0.8 \pm 0.1$ piglets fostered on and $0.6 \pm 0.1$ piglets fostered off per litter $)$ and the number of piglets born below $1 \mathrm{~kg}$ was recorded for each sow after fostering. Sows were restrict fed until d 3 after farrowing and then fed ad libitum until weaning. Average daily feed intake (ADFI) of sows and all piglet mortalities were recorded. Variables were analysed using the UNIVARIATE procedure of SPSS (IBM SPSS Statistics for Windows, v24.0. Armonk, NY) with diet (Control vs. GAA) and parity (1, 2, 3, 4 and $\geq 5$ ) included in a 2 x 5 factorial arrangement, with replicate as a blocking factor and litter as the experimental unit. Binary data, such as percentage of low birth weight piglets and mortality were analysed using Pearson $\chi^{2}$ for the dietary effect.

Results Sows supplemented with GAA had a higher ADFI during lactation, lost less weight during lactation, were heavier at weaning than sows on the control diet, and had less piglets weighing $<1 \mathrm{~kg}$ at fostering $(P<0.05$; Table 1$)$. Number of piglets born alive, piglet pre-weaning mortality and piglet ADG were similar $(P \geq 0.05)$ among treatments.

Table 1. Reproductive performance and litter performance (mean \pm SE) of sows fed either a control (CON) diet, or a diet supplemented with $1 \mathrm{~g} / \mathrm{kg}$ guanidinoacetic acid (GAA) from day 70 of gestation until weaning.

\begin{tabular}{lccccc}
\hline & \multicolumn{3}{c}{ Diet } & \multicolumn{3}{c}{$P$ values } \\
Variable & CON & GAA & Diet (D) & Parity (P) & $\mathrm{D} \times \mathrm{P}$ \\
\hline Sow BW at entry $(\mathrm{kg})$ & $275 \pm 3$ & $280 \pm 3$ & 0.18 & $<0.001$ & 0.031 \\
Sow BW at weaning $(\mathrm{kg})$ & $246^{\mathrm{a}} \pm 3$ & $255^{\mathrm{b}} \pm 3$ & 0.015 & $<0.001$ & 0.12 \\
Sow ADFI $(\mathrm{kg} / \mathrm{d})$ & $7.0^{\mathrm{a}} \pm 0.1$ & $7.3^{\mathrm{b}} \pm 0.1$ & 0.006 & 0.40 & 0.47 \\
Total piglets born & $13.2 \pm 0.3$ & $12.5 \pm 0.3$ & 0.08 & 0.96 & 0.51 \\
Piglets born alive & $12.2 \pm 0.3$ & $11.7 \pm 0.3$ & 0.19 & 0.75 & 0.44 \\
Piglet PFM (\%) & 1.3 & 1.7 & 0.47 & - & - \\
Pigs $<1$ kg at fostering (\%) & $5.5^{\mathrm{a}}$ & $3.4^{\mathrm{b}}$ & 0.019 & - & - \\
Piglet ADG (g/d) & $226 \pm 4$ & $232 \pm 5$ & 0.37 & 0.93 & 0.40 \\
Piglet PWM (\%) & 11.0 & 11.4 & 0.78 & - & - \\
\hline
\end{tabular}

a,b Means within a row with different superscripts differ significantly $(P<0.05)$; BW, body weight; ADFI, average daily feed intake (during lactation); ADG, average daily gain; PFM, pre-foster mortality; PWM, pre-weaning mortality.

Conclusion The results from this study support the hypothesis that addition of $1 \mathrm{~g} / \mathrm{kg}$ GAA to multiparous sow diets improves their lactation feed intake and body condition at weaning but does not significantly improve piglet pre-weaning performance.

Acknowledgements The authors gratefully acknowledge the technical assistance of the Research and Innovation team at Rivalea Australia Pty Ltd.

\section{References}

Brosnan JT, Wijekoon EP, Warford-Woolgar L, Trottier NL, Brosnan ME, Brunton JA and Bertolo RF 2009. The Journal of Nutrition. 139, 1292-1297. 


\section{Black soldier fly technology can convert piggery manure into valuable fertiliser}

$\underline{\text { S N Jenkins }}^{1}$, I S Waite ${ }^{1}$, L K Abbott ${ }^{1}$ and L Wheat ${ }^{2}$

${ }^{1}$ The University of Western Australia, Australia, ${ }^{2}$ Future Green Solutions Pty Ltd, Australia

sasha.jenkins@uwa.edu.au

Application Black Soldier Fly (Hermetia illucens, BSF) farming provides an alternative waste management solution on piggeries by utilising manure to produce a high protein insect meal, whilst the fly castings (frass) are a valuable fertiliser. Its adoption will increase sustainability and productivity whilst safeguarding future food security.

Introduction BSF farming is an emerging industry that provides a low-cost waste management solution for converting agricultural waste into high quality fertiliser (BSF casting residue or frass) and protein (BSF larvae as animal feed) (Bosch et al., 2019; Alattar et al., 2016). However, the BSF frass cannot be developed further in Australia as a fertiliser until the agronomic and economic value of this product is fully evaluated. The objective was to quantify the agronomic benefit of frass derived from piggery manure as an alternative fertiliser for wheat production. It was hypothesised that the frass amendment would increase yield, nutrient content and soil fertility in comparison to other manure derived fertiliser products.

Materials and methods Fresh and stockpiled manure and two graded composts were collected from a piggery in Gingin. Frass was generated from feeding pig manure to BSF larvae at Future Green Solutions (Geraldton, WA). After 2-5 d the resulting BSF pupa was harvested leaving behind the frass residue. A pot experiment was established to compare crop productivity between soil amended with frass and soil amended with four manure products currently on the market (fresh and stockpiled manure, low and high grade composts). Control treatments included a zero fertiliser control, and an addition of synthetic fertiliser (NPS) at rates of 10, 25, 50,75 and $100 \mathrm{~kg} / \mathrm{ha}$. District practice for this area and soil type is $100 \mathrm{~kg} \mathrm{NPS} /$ ha. Wheat (Triticum aestivum L.) was used as the host plant in a randomized block design in four replicates. The pots were maintained at $80 \%$ water holding capacity. Seedlings were thinned to two per pot and grown to maturity before harvest at $118 \mathrm{~d}$. Total shoot dry weight per pot for each treatment was then calculated.

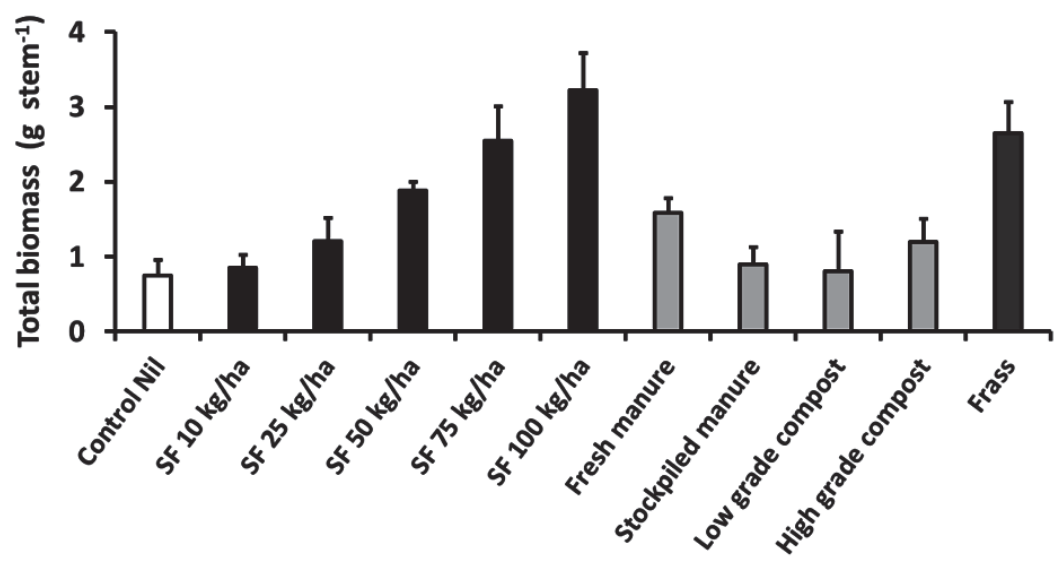

Figure 1. Total biomass production of wheat, in response to no amendment, 5 rates of synthetic fertiliser (SF, applied at $10,25,50,75$ and $100 \mathrm{~kg} / \mathrm{ha}$ ), four conventional manure products (fresh and stockpiled manure, low and high grade composts) and BSF frass. Error bars represent standard error $(\mathrm{n}=4)$.

Results The synthetic fertiliser applications resulted in a typical fertiliser response for grain yield (Figure 1). The BSF frass performed as well as synthetic fertiliser in terms of nutrient provision to wheat crops and yield $(P \leq 0.05)$. In contrast, the total biomass of the plants receiving the conventional manure and compost products were a lot more variable with only the fresh manure treatment having a yield greater than the control.

Conclusion These data show that the use of frass derived from piggery manure as an alternative fertiliser significantly improved overall wheat production when compared to the fresh, stockpiled or composed piggery manure. Adoption of BSF technology on piggeries has potential to increase productivity and profitability via reduced input costs and generation of high quality products from manure that provide additional revenue streams.

Acknowledgements Supported in part by Australian Pork Limited and the Department of Agriculture.

\section{References}

Alattar MA, Alattar FN and Popa R 2016. Plant Science Today. 3, 57-62.

Bosch G, van Zanten HHE, Zamprogna A, Veenenbos M, Meijer NP, van der Fels-Klerx HJ and van Loon JJA 2019. Journal of Cleaner Production. 222, 355-363. 


\section{Quantifying use of electricity to heat piglet creep areas in farrowing rooms}

L J Johnston $^{1}$, K T Sharpe ${ }^{1}$, M H Reese ${ }^{1}$, E S Buchanan ${ }^{1}$, J E Tallaksen ${ }^{1}$, A M Hilbrands ${ }^{1}$, K A Janni ${ }^{2}$, B Hetchler ${ }^{2}$ and $\mathrm{Y} \mathrm{Li}^{1}$

${ }^{1}$ West Central Research and Outreach Centre, University of Minnesota, USA, ${ }^{2}$ Bioproducts and Biosystems

Engineering, University of Minnesota, USA

johnstlj@umn.edu

Application Incandescent electric heat lamps used to warm piglet creep areas consumed over 50\% of all the electricity supplied to the farrowing operations studied. Replacing incandescent heat lamps with electric heat mats may provide a practical approach to reduce electricity consumption and improve the environmental footprint of pork production.

Introduction Consumers and food retailers increasingly desire food products with a reduced environmental footprint. One of many approaches to reduce the environmental footprint of pork production is to reduce the use of fossil fuels. In many parts of the world, electricity is generated from combustion of fossil fuels which releases greenhouse gases. If pork producers could reduce their consumption of electricity, a reduction in greenhouse gas emissions could be realized. A first step in reducing electrical use is to quantify what processes and equipment use the most electricity. We surmised that piglet heat lamps would be a large consumer of electricity. Therefore, our objectives were to quantify the amount of electricity used by incandescent heat lamps to warm piglet creep areas in confinement farrowing systems and investigate use of heat mats as a method to potentially reduce use of electricity.

Material and methods Electrical use and weaned pig production were monitored over several years at three different swine farrowing operations. The initial phase comprised two years of data collected from two commercial farrowing operations (Commercial farm A, 2600 and Commercial farm B, 3300 breeding sows). The second phase included data collected from farrowing rooms at the University of Minnesota Swine Research Farm (60 breeding sows) using two contemporary farrowing groups (University group A and B). Phases 1 and 2 used incandescent heat lamps (125 W) in the piglet creep area. In phase three, data were collected in a farrowing room at the University (same room as used in phase 2) equipped with electric heat mats (Innovative Heating Technologies, Inc., Manitoba, Canada) in place of incandescent heat lamps. All farrowing accommodations housed sows and litters in farrowing stalls and heat lamps and mats operated continuously from farrowing to weaning. Electrical loads related to pig production were measured using stand-alone data loggers (HOBO UX120-006M, Onset Computer Corporation, Bourne, MA) and current sensors (CR Magnetics, St. Louis, MO and Magnelab Inc., Longmont, CO) at the circuit panel box (Sharpe et al., 2018). Data for weaned pig production were captured from routine production records of the farms.

Results Electricity use ranged from about 5 to $7.25 \mathrm{kWh}$ (Table 1) per weaned pig produced. Electricity consumption was consistent across the large commercial farms and the small, near-commercial research farm. Electric heat lamps consumed over half of all the electricity used on the commercial sow farms and $75 \%$ or more of the electricity used in the farrowing rooms of the University research farm. Clearly, heat lamps used to warm piglet creep areas are a large user of electricity in farrowing operations and represent a segment of energy use that might be improved on sow farms. In the farrowing room equipped with electric heat mats, 165 weaned pigs were produced and $2.84 \mathrm{kWh}$ of electricity/weaned pig $(61 \%$ of electricity used in the farrowing room) were required to operate the heat mats. Use of heat mats reduced electricity consumption to warm piglets by about $50 \%$. This reduction in electricity use equates to a reduction of about $1.65 \mathrm{~kg} \mathrm{CO} 2 \mathrm{e}$ of greenhouse gases per weaned pig (U.S. EPA 2018).

Table 1. Electricity use by heat lamps in piglet creep areas of farrowing stalls

\begin{tabular}{|c|c|c|c|}
\hline Location & $\begin{array}{l}\text { Number of weaned } \\
\text { pigs produced }\end{array}$ & $\begin{array}{c}\text { Electricity used } \\
(\mathrm{kWh} / \text { weaned pig) }\end{array}$ & $\begin{array}{c}\text { Proportion of total } \\
\text { electricity for heat lamps }\end{array}$ \\
\hline Commercial sow farm $\mathrm{A}$ & 116838 & 6.60 & $58 \% \%^{\mathrm{a}}$ \\
\hline Commercial sow farm B & 175344 & 7.27 & $61 \%{ }^{a}$ \\
\hline University group A & 169 & 5.06 & $75 \% \mathrm{~b}$ \\
\hline University group B & 153 & 6.65 & $83 \% \mathrm{~b}$ \\
\hline
\end{tabular}

Conclusion Incandescent heat lamps used to warm piglet creep areas used over $50 \%$ of all electricity consumed in a farrowing operation. Attempts to reduce the environmental footprint of farrowing operations should focus on reducing electricity use for heating piglet creep areas. Electric heat mats may provide a practical solution to reduce electricity use on farrowing operations.

Acknowledgements Authors thank the Minnesota Environment and Natural Resources Trust Fund for funding this work.

\section{References}

Sharpe KT, Reese MH, Buchanan ES, Tallaksen JE, Janni KA and Johnston LJ 2018. Applied Engineering in Agriculture. 34, 857-864.

U.S. EPA 2018. https://www.epa.gov/energy/emissions-generation-resource-integrated-database-egrid 
Trends in the environmental impacts from the Australian pork industry: 1980-2020

$\underline{\text { K J L Watson }}^{1}$, S G Wiedemann ${ }^{1}$, L Biggs ${ }^{1}$ and E J McGahan ${ }^{1}$

${ }^{1}$ Integrity Ag \& Environment, Australia

kalinda.watson@integrityag.net.au

Application This study demonstrated significant improvements in environmental performance in the pork industry over 40 years, highlighting trends that will influence future environmental performance of the industry. This information will inform pork consumers interested in the overall environmental efficiency of the industry.

Introduction Australian pig production has transitioned from small farm enterprises to large scale, specialist pig farming operations in the second half of last century (Gardner, 1990), which has profoundly influenced production efficiency (e.g. weaning rates, live weight produced per sow, average daily gain and feed conversion ratio - FCR). The study investigated the "cradle-to-farm gate" trend in environmental performance of the Australian pork industry over 40 years. We hypothesised that productivity improvements and changes in housing and manure management would also lead to lower environmental impacts from pork over the study period.

Material and methods The study used a life cycle assessment (LCA) approach and reported impacts per kilogram of pork (live weight - LW) at 10 year intervals from 1980 to 2010, and impacts were projected for 2020. Greenhouse gas (GHG) emissions were calculated using Global Warming Potentials from Working Group 1 (2007). GHG emissions arising from land use (LU) and direct land use change (dLUC) were calculated and reported separately following the guidance of ISO/TS 14067. Energy demand and freshwater consumption was assessed from inventory data, and water stress was assessed using the method of Pfister et al. (2009). Modelling was conducted using SimaPro 8.5 (PRé Sustainability, Amersfoot, the Netherlands).

Results The analysis showed that over the four decades since 1980 there has been a decrease in GHG emission intensity, excluding land use (LU) and direct land use change (dLUC) emissions, from 10.6 to $3.3 \mathrm{~kg} \mathrm{CO}$-e/ $\mathrm{kg} \mathrm{LW}$. GHG emissions associated with LU and dLUC were estimated to have declined by $89 \%$ from $2.94 \mathrm{~kg} \mathrm{CO}$-e/ $/ \mathrm{kg} \mathrm{LW}$ in 1980 to a projected $0.32 \mathrm{~kg} \mathrm{CO}$-e/kg LW in 2020. Freshwater consumption decreased from $441 \mathrm{~L} / \mathrm{kg} \mathrm{LW}$ in 1980 to a projected $90 \mathrm{~L} / \mathrm{kg} \mathrm{LW}$ in 2020. Water stress followed a similar trend, decreasing from $442 \mathrm{~L} \mathrm{H}_{2} \mathrm{O}-\mathrm{e} / \mathrm{kg} \mathrm{LW}$ in 1980 to a projected $79 \mathrm{~L} \mathrm{H}_{2} \mathrm{O}-$ e/kg LW in 2020. Fossil energy use decreased from $34 \mathrm{MJ} / \mathrm{kg} \mathrm{LW}$ in 1980 to a projected $14 \mathrm{MJ} / \mathrm{kg} \mathrm{LW}$ in 2020. Land occupation decreased from $31 \mathrm{~m}^{2} / \mathrm{kg} \mathrm{LW}$ in 1980 to a projected $11 \mathrm{~m}^{2} / \mathrm{kg} \mathrm{LW}$ in 2020 . Improvements were principally driven by improved herd productivity, changes in housing and manure management, and improved feed production systems. In the pig production system, improved FCR reduced upstream impacts from feed production and reduced relative manure production, and related environmental impacts. Concurrently, improvements in feed grain production systems resulted in lower impacts per tonne of feed grain produced, which was related to reduced tillage, higher yields and a decrease in the proportion of irrigation used for grain production.

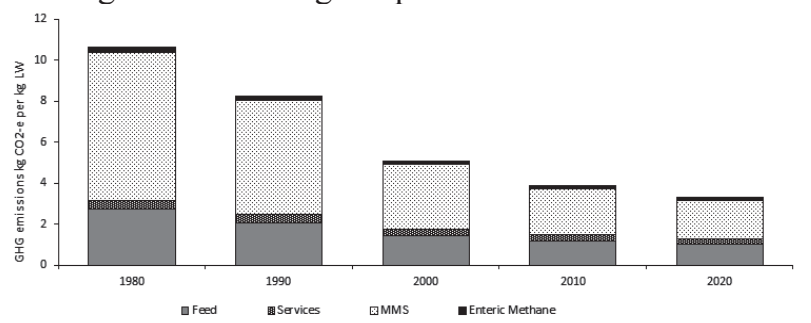

Figure 1. Changes in GHG (excluding LU and dLUC) from the production of $1 \mathrm{~kg}$ LW of pork from 1980-2020.

Conclusion There has been a significant improvement in productivity, with more LW sold per sow, and lower FCRs over 40 years in the Australian pork industry. Over the four decades since 1980 this has contributed to a $69 \%, 58 \%, 80 \%$ and $63 \%$ reduction in GHG emissions, fossil fuel use, water consumption and land occupation, respectively, supporting the hypothesis. While further improvement in FCR is expected to result in lower environmental impacts, this will be limited by practical and biological potential, suggesting that adoption of improved manure management (i.e. expansion of biogas and closed loop technologies) and utilisation of low impact feed sources and by-products are also important in order for the industry to position itself as a low impact meat product.

Acknowledgements Supported in part by Australian Pork Limited. Thanks is expressed to industry members who contributed data to this project.

\section{References}

Gardner JAA 1990. In Pig Production in Australia: 2nd Edition (Eds: Gardner JAA, Dunkin AC, Lloyd LC). Butterworths Pty Limited, Australia. pp. 7-11

Pfister S, Koehler A and Hellweg S 2009. Environmental Science \& Technology. 43, 4098-4104.

Working Group 1 2007. In Climate change 2007 - the physical science basis: Working group I contribution to the fourth assessment report of the IPCC (Eds: Solomon S, Qin D, Manning M, Chen Z, Marquis M, Averyt K, Tignor M and Miller H) Cambridge University Press, UK. 


\section{Effects of weaning age and antibiotic use on pig performance in a commercial system}

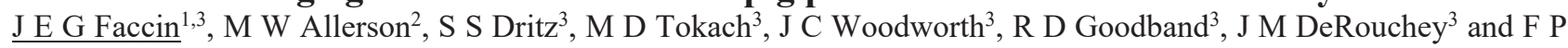
Bortolozzo $^{1}$

${ }^{1}$ Federal University of Rio Grande do Sul, Brazil, ${ }^{2}$ Holden Farms, USA, ${ }^{3}$ Kansas State University, USA

jamilfaccin@gmail.com

Application Increasing weaning age and antibiotic use exhibit an important impact in the wean-to-finish performance maximising growth rate, minimising losses, and thus, improving the volume of pork produced per pig weaned. Since swine production has followed a trend of reducing use of antibiotics, other management strategies that influence growth and mortality, such as older weaning ages, are needed to maintain economically competitiveness of pork production.

Introduction More than a decade ago, a study conducted by Main et al. (2004) assessed the impact of weaning age on performance in a commercial system. The range of weaning ages used in that work was from 12 to $21.5 \mathrm{~d}$, which was very applicable to the swine production of that time. Most of the findings of that study exhibited linear improvements such as growth rate, mortality, and weight sold per pig weaned, reinforcing the necessity to evaluate an older range of weaning ages. Likewise, as pressure to lower antibiotic use increases, a renewed look at weaning age appears warranted for production systems. Therefore, the objective of this study was to determine the effects of weaning age and antibiotic use on pig performance in a commercial production system.

Material and methods A total of 2184 barrows and gilts $\left(\mathrm{DNA}^{\circledR} \times \mathrm{L}^{\mathrm{B}} 2^{\circledR}\right)$ from four weaning batches were used in a study from weaning to market. A 3 x 2 factorial arrangement was used. Litters were weaned at 18, 19, 21, 22, 24, or 25 d of age. The treatments included weaning age $(18.5,21.5$ or $24.5 \mathrm{~d})$ and antibiotic use (AB or ABF). There were 14 replicate pens per treatment and 26 pigs per pen (13 barrows and 13 gilts). Pigs were ear tagged at birth to guarantee the exact age at weaning. Pigs were weaned from a 4000 sow farm and were placed in pens by weaning age with pens randomly assigned to $\mathrm{AB}$ or $\mathrm{ABF}$. Pigs assigned to $\mathrm{AB}$ had access to a diet containing 400 ppm of chlortetracycline (CTC) from d 10 to 24 post-weaning, and after a PRRS outbreak (experienced by all pigs) at week 7 post-weaning, they were again medicated via drinking water for five consecutive days with CTC (22 $\mathrm{mg} / \mathrm{kg}$ of body weight per day). Individual pigs on both treatments were treated with antibiotics as required. Data were analysed with GLIMMIX procedure (SAS Institute Inc., Cary, NC, USA) and polynomial contrasts were implemented. Pens were considered as the experimental unit.

Results For the first 42 d post-weaning, increasing weaning age reduced the number of pigs treated with injectable antibiotic (quadratic, $P=0.004)$, but AB use did not influence this variable $(P=0.626)$. At weaning and $197 \mathrm{~d}$ of age, increasing weaning age resulted in greater body weight $(\mathrm{BW})$ with slopes of $0.220 \mathrm{~kg}$ and $0.675 \mathrm{~kg}$, respectively for each day increase in weaning age (linear, $P<0.001$ ). From weaning to $197 \mathrm{~d}$ of age, increasing weaning age increased average daily gain $(11 \mathrm{~g} / \mathrm{d}$ of weaning age; linear, $P<0.001)$ and the same effect was found for $\mathrm{AB}(14 \mathrm{~g} / \mathrm{d} ; P=0.020)$. Interactions (linear, $P<0.045)$ were found for average daily feed intake and feed efficiency. Pigs with access to AB in feed and water had lower total losses $(2.7 \%$ less mortality + removal; $P<0.001)$. Increasing weaning age also marginally decreased total losses (Losses $=-0.21 \%$ per day increase in weaning age; linear, $P=0.097$ ). The weight sold (at $197 \mathrm{~d}$ of age) per pig weaned was increased by increasing weaning age (an additional $705 \mathrm{~g}$ for each day increase in weaning age; linear, $P=$ 0.050 ) and by using $\mathrm{AB}$ in feed and water (an addition of $4.6 \mathrm{~kg} / \mathrm{pig} ; P=0.019$ ).

Conclusion Increasing weaning age linearly improved pig performance. Relatively short term use of antibiotics reduced mortality and removals. Driven by a reduction of the percentage of losses and performance improvement, the weight sold per pig weaned was maximised when pigs were weaned at older ages and antibiotics were used in the feed and water.

Acknowledgements The authors gratefully acknowledge funding from Holden Farms, Inc., Northfield MN, USA.

\section{References}

Main RG, Dritz SS, Tokach MD, Goodband RD and Nelssen JL 2004. Journal of Animal Science. 82, $1499-1507$. 


\section{The effect of Lactobacillus and yeast ferment-based additives on post-weaning diarrhoea in enterotoxigenic Escherichia coli challenged piglets}

T Laird $^{1}$, J P Mansfield ${ }^{1}$, S Sahibzada ${ }^{1}$, D L Turpin ${ }^{1}$, J R Pluske ${ }^{1}$, S Abraham ${ }^{1}$ and M O’Dea ${ }^{1}$

${ }^{1}$ Murdoch University, Australia

t.laird@murdoch.edu.au

Application This study indicates that pig producers may see an increased average daily gain (ADG) when feeding SFP products to pigs recovering from PWD.

Introduction Post-weaning diarrhoea (PWD) in piglets is a financial burden to the swine industry with substantial economic loss due to the associated reduced growth performance, increase in treatment costs and increased mortality rates (Van Breda et al., 2017). Lactobacillus acidophilus fermentation products (LFP) and Saccharomyces cerevisiae fermentation products (SFP) supplemented in animal feed have displayed increased ADG (Feye et al., 2016) and reduced shedding of E. coli in healthy pigs (Lan et al., 2016). This study hypothesised that these feed additives would reduce shedding of enterotoxigenic Escherichia coli (ETEC) and would lessen the severity of PWD, resulting in increased ADG in ETEC-challenged piglets.

Material and methods Male pigs ( $\mathrm{n}=80$ ) of three weeks of age and weighing $6.04 \pm 1.07 \mathrm{~kg}$ were transported to Murdoch University and randomly allocated into pens on the basis of weight. Each pen contained four pigs, with four pens assigned to each of the five treatment groups. The five treatment groups were; (1) basal diet (BD) (negative control), (2) BD with 3000 ppm zinc oxide ( $\mathrm{ZnO}$; positive control), (3) BD with 2000 ppm LFP (SynGenX, Diamond V, Cedar Rapids, IA, USA), (4) BD with 2000 ppm SFP (Diamond V XPC, Diamond V, Cedar Rapids, IA, USA), and (5) BD with 2000 ppm LFP and 2000 ppm SFP (LFP + SFP). All pigs received feed and water ad libitum. Pigs were orally dosed with ETEC on d 6 and 7 after arrival and were monitored for a further 4 weeks. Faecal consistency scores were recorded daily and pig weights and feed intake measured weekly. Pooled pen faecal samples were taken on d 0 (infection), 1, 2, 3, 4 and 7. Faeces were homogenised in PBS and dilutions plated onto 5\% sheep blood agar. Rectal swabs were also taken on these days and additionally on d 9 and 27. Rectal swabs were manually streaked onto sheep blood agar and the presence of ETEC detected and scored as no, low, moderate or pure growth. Data were statistically analysed using a linear regression and generalised linear models in SPSS (IBM SPSS Statistics for Windows, v24.0. Armonk, NY).

Results Two days after infection the cumulative number of pigs with predominant or pure growth of E. coli was $38 \%$, the cumulative number with any haemolytic $E$. coli shedding was $87 \%$ and the cumulative number with diarrhoea scores above 4 was $70 \%$. A significant difference $(P<0.05)$ in ADG between groups 2 and 4 was seen in $\mathrm{d} 17$ to 27 with the mean ADG being 554 and $690 \mathrm{~g} / \mathrm{d}$, respectively. There was no difference $(P>0.05)$ in the ADG between other groups within this time period and no difference $(P>0.05)$ in ADG when assessed over the full duration of the study (Table 1). Following infection, pigs fed diet (2) peaked in ETEC faecal shedding on d 1, diets (4) and (5) on d 2, and the other two treatments showed increasing ETEC cfu/g faeces up until d 3, with no significant association observed between the diet and ETEC shedding.

Table 1. Average daily gain of piglets offered diets supplemented with Lactobacillus acidophilus fermentation products (LFP) and Saccharomyces cerevisiae fermentation products (SFP), challenged with enterotoxigenic E. coli at d 0.

\begin{tabular}{lccccc}
\hline & \multicolumn{3}{c}{ Average daily gain (g/d) } \\
& d -5 to 2 & d 3-9 & d 10-16 & d 17-27 & Overall d -5 to 27 \\
\hline Basal diet (BD; -ve control) & 42.9 & 175.7 & 347.9 & $601.5^{\text {ab }}$ & 311.9 \\
BD + 3 000 ppm ZnO (+ve control) & 67.9 & 230.9 & 337.8 & $553.9^{\mathrm{a}}$ & 312.3 \\
BD + 2 000 ppm LFP & 58.6 & 201.8 & 379.8 & $626.9^{\text {ab }}$ & 336.0 \\
BD + 2 000 ppm SFP & 49.8 & 175.2 & 317.1 & $689.7^{\mathrm{b}}$ & 334.1 \\
BD + 2 000 ppm LFP + SFP & 60.2 & 239.9 & 381.7 & $672.1^{\text {ab }}$ & 359.2 \\
$P$ value & N.S. & N.S. & N.S. & $<0.05$ & N.S. \\
\hline
\end{tabular}

Conclusion The addition of SFP to a basal diet resulted in a significant increase in ADG when compared to a basal diet with $3000 \mathrm{ppm}$ zinc oxide over the period of d 17 to 27 . No effects on faecal haemolytic E. coli shedding in ETECchallenged pigs were noted, however a combination of SFP and LFP in the diet decreased the duration of shedding by one day. Further processing of samples will include 16S RNA sequencing to determine treatment effects on faecal microbiota.

\section{References}

Feye KM, Anderson KL, Scott MF, McIntyre DR and Carlson SA 2016. Poultry Science. 95, 2902-2910.

Lan RX, Koo JM, Lee SI and Kim IH 2016. Journal of Animal Science. 94 (Supp 2), 71.

Van Breda LK, Dhungyel OP, Ginn AN, Iredell JR and Ward MP 2017. PLoS One. 12, e0172528. 


\section{A multiplex molecular assay for Glaesserella australis}

$\mathrm{S} \mathrm{Yee}^{1}, \mathrm{~N}$ Nahar ${ }^{1}$, E T Lobo ${ }^{1}, \mathrm{~S}$ Jenkin ${ }^{2}$ and $\mathrm{C} \mathrm{Turni}^{1}$

${ }^{1}$ Queensland Alliance for Agriculture and Food Innovation, The University of Queensland, Australia, ${ }^{2}$ Apiam Animal Health, Australia

C.Turni1@uq.edu.au

Application The development of a PCR for Glaesserella australis will result in its correct identification and therefore improve its prevention and control.

Introduction A new bacterial species, consisting of 17 isolates, has been previously identified (Turni et al., 2018) and has been unofficially named Glaesserella australis. The isolates collected came from different disease scenarios. Some isolates were taken from lungs that displayed acute consolidation of dorsal lung lesions, which affected approximately $50 \%$ of the lung with no gross abscesses or pleurisy and were from pigs aged 12 to 20 weeks that died on farm. From some of these lungs, the only bacterial species isolated was G. australis. The other isolates came from lungs collected at the abattoir that displayed lung lesions, lung abscesses and pleurisy while on farm no symptoms or only mild respiratory symptoms were observed. The lesions observed at the abattoir were very similar to lesions of Actinobacillus pleuropneumoniae in appearance and it was hypothesised that due to the similarity in lesions and time of onset of the disease caused by $G$. australis and A. pleuropneumoniae, it might have been that these lesions at the abattoir had been attributed to the wrong bacterial species. To be able to do a study on the abundance of this species, a species-specific PCR has been developed, which is a multiplex PCR for the detection of G. australis, Pasteurella multocida and A. pleuropneumoniae. The hypothesis of this study was that the multiplex PCR developed would be specific for G. australis without amplifying any related strains.

Material and methods A total of 26 isolates of $G$. australis including the original 17 isolates and a further 9 field isolates collected from lungs at the abattoir were used. Isolates of the other two species in this PCR were also tested with 15 reference serovar strains and one field isolate of $A$. pleuropneumoniae and 16 reference serovar strains plus the type strain of $P$. multocida. To assure specificity, 47 related species including type strains and field isolates were tested in the PCR. DNA was extracted by suspending a loopful of bacteria into $\mathrm{H}_{2} \mathrm{O}$, heating and boiling twice and spinning at $13000 \mathrm{x} g$ for 2 minutes. The $24 \mu \mathrm{L}$ PCR reaction mix contained $1.5 \mathrm{mM} \mathrm{MgCl}_{2}, 10 \mathrm{mM}$ Tris, $50 \mathrm{mM} \mathrm{KCl,} 0.24 \mathrm{mM} \mathrm{dNTPs}, 0.3$ $\mu \mathrm{M}$ of each primer (Table 1), $0.5 \mathrm{U}$ of Taq and $1 \mu \mathrm{L}$ of template. The reaction mix was run for one cycle at $94^{\circ} \mathrm{C}$ for 2 min and then for 30 cycles at $94^{\circ} \mathrm{C}$ for $30 \mathrm{sec}, 65^{\circ} \mathrm{C}$ for $30 \mathrm{sec}, 72^{\circ} \mathrm{C}$ for $2 \mathrm{~min}$. After this followed a final cycle at $72^{\circ} \mathrm{C}$ for 10 min. Furthermore, a total of 16 swabs were sampled from 15 lungs that were sampled at the abattoir, cultured and resulting isolates tested by PCR.

Table 1. Primers used in the assay

\begin{tabular}{|c|c|c|c|}
\hline Primers & & Amplicon & Reference \\
\hline LPF & 5' - AAG GTT GAT ATG TCC GCA CC -3' & \multirow{2}{*}{951} & \multirow{2}{*}{ Gram and Ahrens 1998} \\
\hline LPR & 5' - CAC CGA TTA CGC CTT GCC A - 3' & & \\
\hline PM1231F & 5' - AGA AAG CAC ATG ACC AAA GGG - 3' & \multirow{2}{*}{601} & \multirow{2}{*}{ Liu et al., 2004} \\
\hline PM1231R & 5' - GCA GCT ACT CGC AGA AGG TT - 3' & & \\
\hline GA F & 5' - AAG ATG ATG ATC GCC CAA TCG 3' & \multirow{2}{*}{449} & \multirow{2}{*}{ This study } \\
\hline GAR1 & 5' - CCA CGA GAA GCA AGA ACA TCT TTG ATC - 3' & & \\
\hline
\end{tabular}

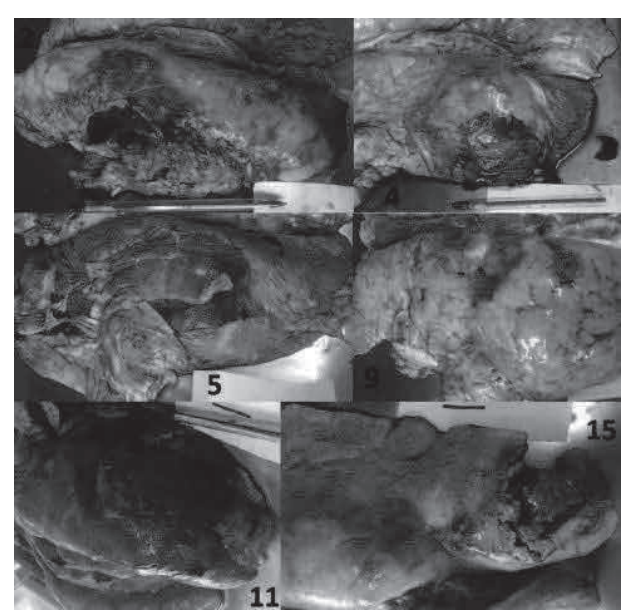

Figure 1. Showing the six lungs with lesions from which $G$. australis was isolated.
Results The multiplex PCR amplified all the $G$. australis, the $A$. pleuropneumoniae and $P$. multocida reference, type and field strains. The PCR was very specific and did not amplify any of the related strains, be that type strains or field strains. The sampling at the abattoir yielded pure culture of $G$. australis from six lungs. The appearance of the lesions of some lungs resembled A. pleuropneumoniae lesions (Figure 1).

Conclusion The new multiplex is specific and can identify G. australis in pure culture. The assay would appear to be a useful addition to the suite of diagnostic tools for pig respiratory disease diagnostics.

Acknowledgements Supported in part by Australian Pork Limited.

\section{References}

Gram T and Ahrens P 1998. Journal of Clinical Microbiology. 36, 443-448.

Liu D, Lawrence ML and Austin FW 2004. Journal of Microbiological Methods. 58, 263-267.

Turni C, Giang N, Wu Y, Omaleki L, Blackall PJ and Christensen H 2018. In Australian Society for Microbiology Annual Scientific Meeting. Abstract 385. 
The association between sire immune competence and antibody response of progeny

$\mathrm{J}$ Harper $^{1}, \mathrm{~K}$ L Bunter ${ }^{2}$, S Hermesch ${ }^{2}$ and B C Hine ${ }^{3}$

${ }^{1}$ Rivalea Australia Pty Ltd, Australia, ${ }^{2}$ Animal Genetics and Breeding Unit, a joint venture of NSW Department of Primary Industries and University of New England, University of New England, Australia, ${ }^{3}$ CSIRO Agriculture and Food, Australia

jharper@rivalea.com.au

Application Selection for improved immune competence in sires has the potential to improve antibody response to immune challenges and improve survival of progeny in commercial piggery environments.

Introduction Procedures using commercially available test antigens have been developed to assess the immune competence phenotype of pigs (Harper et al., 2017), which combines measures of an animal's ability to mount both antibody-mediated (Ab-IR) and cell-mediated immune response (Cell-IR). In previous work, Harper et al. (2018) demonstrated that sire differences in Cell-IR, but not Ab-IR, were associated with breeding values for pre-weaning survival of their progeny. We speculated that extensive post-weaning vaccination schedules for progeny potentially influenced associations between sire immune competence phenotypes and breeding values for post-weaning survival observed. The hypothesis of this study was that the antibody response of progeny reared in commercial environments would be favourably associated with immune competence phenotypes of their sires, when assessed using common model antigens.

Material and methods Mature boars, located in a commercial artificial insemination (AI) centre (Primegro ${ }^{\mathrm{TM}}$ Genetics, Corowa, NSW) in southern NSW, Australia were assessed for overall immune competence using a model antigen, as outlined in Harper et al. (2018). These boars were, at the time of testing, being used to sire purebred progeny. A subset of 90 purebred progeny, grown in the commercial piggery environment, not previously vaccinated against tetanus toxoid (TT) and with low likelihood of natural exposure to Clostridium tetani, were available from boars with predominantly high or low Ab-IR and Cell-IR immune response phenotypes, to test for their own ability to mount an antibody response to the same model antigen. On d 0 and 21, progeny were vaccinated subcutaneously, with $2 \mathrm{ml}$ of $\mathrm{Ultravac}^{\circledR} 5 \mathrm{in} 1$ (Zoetis Inc., Rhodes, NSW), containing tetanus toxoid (TT) antigen. On d 30, when progeny were on average $136 \pm 6.8 \mathrm{~d}$ of age, blood was collected. Serum samples were tested, on a single plate, for TT-specific IgG antibody levels using an indirect enzyme-linked immunosorbent assay (ELISA), as described previously by Miller et al. (2013). The antibody response of individual animals was represented as a sample to positive ratio (SP):

$$
\mathrm{SP}=\frac{\left(\text { Sample optical density }- \text { Negative control mean }{ }^{a}\right)}{\left(\text { Positive control }^{b}-\text { Negative control mean }^{a}\right)}
$$

${ }^{a}$ mean optical density of negative control samples; ${ }^{b}$ highest sample optical density from d 30

The effect of sire immune group was estimated by fitting a mixed model, where the combined grouping (Ab-IR-Cell-IR) of sires was fitted as a fixed effect, and sire was treated as a random effect, using the 'Ime' procedure in R (R Core Team 2016).

Results The differences in progeny antibody response between sire immune groups are presented in Table 1. Progeny from low-low immune competence sires tended $(P=0.06)$ to have lower antibody response to the same model antigen used to assess their sires for immune competence.

Table 1. Number of progeny tested and mean $\pm \mathrm{SE}$ of sample to positive ratio (SP) for sire immune grouping - group codes represent the concatenation of Ab-IR and Cell-IR respectively.

\begin{tabular}{lcccc}
\hline Sire Immune Group & Low - Low & Low - High & High - Low & High - High \\
No. Progeny & 40 & 5 & 5 & 40 \\
SP & $0.70 \pm 0.02$ & $0.81 \pm 0.05$ & $0.82 \pm 0.06$ & $0.75 \pm 0.03$ \\
\hline
\end{tabular}

Conclusion This study demonstrated that the immune competence phenotype of sires was reflected by the ability of their progeny to mount an antibody response. This result, in conjunction with the favourable association of enhanced immune competence and survival of progeny, previously reported by Harper et al. (2018), provides encouraging evidence that immune competence phenotype testing could be conducted in young pigs, to genetically improve the immune competence of commercial pig herds.

Acknowledgements Supported in part by Australian Pork Limited, Pork CRC Limited and Rivalea Australia Pty Ltd

\section{References}

Harper J, Bunter KL, Hermesch S, Hine BC and Collins AM 2017. Animal Production Science. 57, 2464-2464.

Harper J, Bunter KL, Hine BC, Hermesch S and Collins AM 2018. Proceedings of the World Congress on Genetics Applied to Livestock Production. 11, 368.

Miller YJ, Collins AM, Emery D, Begg DJ, Smits RJ and Holyoake PK 2013. Animal Production Science. 53, 46-51.

R Core Team 2016. R: A language and environment for statistical computing. R Foundation for Statistical Computing, Vienna, Austria. 
Faecal microbiota transplantation during lactation had no effect on piglets to weaning T L Nowland ${ }^{1}$, W Y Low ${ }^{1}$, V A Torok ${ }^{2}$, M D Barton ${ }^{3}$, K J Plush ${ }^{4}$ and R N Kirkwood ${ }^{1}$

${ }^{1}$ The University of Adelaide, Australia, ${ }^{2}$ South Australian Research and Development Institute, Australia, ${ }^{3}$ University of South Australia, Australia, ${ }^{4}$ SunPork Group, Australia

tanya.nowland@adelaide.edu.au

Application Faecal microbiota transplantation has the potential to be used as an alternative to antibiotics when treating enteric diseases in pigs. Although this experiment had no effect on pre-weaning piglet microbiome, new information was gained to further our understanding of the role of the intestinal microbiota in piglets.

Introduction Antimicrobial use in animal production and the potential development of bacterial resistance is a growing global concern. Therefore, the development of non-antimicrobial techniques to prevent or treat animal disease need to be explored. One such method that has proven efficacy in the treatment of enteric diseases in humans is faecal microbiota transplantation (FMT) (Bakken et al., 2011). However, little research evidence exists for its application in treating enteric diseases in pigs. The objective of this study was to determine whether neonatal antimicrobial treatment affects the faecal microbiota and whether FMT can restore balance in microbiota composition. It was hypothesised that (1) animals treated with the antibiotic ceftiofur (cf) would undergo an alteration in gut microbiota and (2) the dosing of these animals with faeces from non-treated animals would restore gut microbiota with resemblance to untreated animals.

Material and methods Two piglets per sow were selected arbitrarily to be focus piglets and were assigned to one of four treatments: cffresh $(\mathrm{n}=6)$ received cf $(3 \mathrm{mg} / \mathrm{kg}$ intramuscular, Excenel $(50 \mathrm{mg} / \mathrm{mL}$ Ceftiofur hydrochloride), Zoetis Inc., Rhodes, NSW) at $\mathrm{d} 7$ and fresh FMT at d 13; cffrozen $(\mathrm{n}=7)$ received $\mathrm{cf}$ at $\mathrm{d} 7$ and frozen FMT at $\mathrm{d} 13$; $\mathrm{cf}(\mathrm{n}=8)$ received $\mathrm{cf}$ at $\mathrm{d} 7$ and no FMT; and nocf ( $\mathrm{n}=5$ ) received no $\mathrm{cf}$ and no FMT. Faecal samples were collected from focus pigs at 7, 13 and $18 \mathrm{~d}$ of age to assess faecal microbiota population structure and diversity using $16 \mathrm{~S}$ rRNA amplicon analysis. For frozen FMT, faeces were collected from 8 clinically healthy $14 \mathrm{~d}$ old donor pigs. After collection, faeces were blended $1: 2$ with saline, with glycerol added to $10 \%$, and stored at $-80^{\circ} \mathrm{C}$ until required. Faecal samples for fresh FMT were collected from the same donor pigs and blended 1:2 in saline. Donor piglets had no previous contact with antibiotics or antibiotic treated piglets. All faecal blends were brought to room temperature before being delivered by gavage at $3 \mathrm{ml} / \mathrm{kg}$. Piglets were fasted for $3 \mathrm{~h}$ before FMT to minimise gastric acidity. The V3-V4 region of the 16S rRNA gene ( 460bp) was sequenced using the Illumina MiSeq platform, following a standard protocol. The software Mothur, v1.40.5 (Michigan, USA) was used to obtain rarefaction curve, Shannon diversity and Chao estimates (alpha diversity) from the profiling data. Bray-Curtis measures of similarity (beta diversity) were calculated to examine similarities between faecal bacterial communities following standardisation and fourth-root transformation of data (PRIMER-E v6, Plymouth, United Kingdom). Analysis of similarity (ANOSIM) tested if faecal microbial communities were significantly different between treatments and age.

Results Using Shannon Index as a measure of bacterial diversity within each sample, there was a significant increase in diversity as the piglets aged from 7 to $18 \mathrm{~d}(P<0.05)$. However, there was no significant effect of treatment in richness and diversity between all treatment groups. There were significant differences in faecal microbial communities at the phyla, class, order, family and genus level between 7 and 13 , as well as, 7 and $18 \mathrm{~d}$ old piglets $(P<0.05)$, regardless of treatment. No significant effect of antibiotic and FMT was observed.

Conclusion Significant differences in faecal bacterial communities associated with age were observed. However, the finding that animals treated with the antibiotic ceftiofur had no reduction in richness or diversity in these young piglets was unexpected, as other studies have shown that antibiotics reduced gut microbial communities (Janczyk et al., 2007). The finding that FMT had no influence on the faecal microbiota of piglets is possibly due to the fact that the donor faeces used was not different enough from the host to elicit a change, especially as no effect resulted from antibiotic treatment. Further investigation into the use of FMT post weaning with faeces from older animals may provide more insight, additionally, the use of a greater number of animals is likely necessary.

Acknowledgements Supported in part by Australian Pork Limited and the Australian Government Department of Agriculture and Water Resources. The authors would like to thank Sophia Ward and Zoe Pfeiffer for their help throughout the experiment.

\section{References}

Bakken JS, Borody T, Brandt LJ, Brill JV, Demarco DC, Franzos MA, Kelly C, Khoruts A, Louie T, Martinelli LP, Moore TA, Russell G, Surawicz C and Fecal Microbiota Transplantation Workgroup 2011. Clinical Gastroenterology and Hepatology. 9, 1044-1049.

Janczyk P, Pieper R, Souffrant WB, Bimczok D, Rothkotter HJ and Smidt H 2007. The ISME Journal. 1, 180-183. 


\section{Rapid and easy extraction method using cellulose dipstick in oral fluid samples}

A R dela Cruz $^{1}$, J Meers ${ }^{1}$, T S Barnes ${ }^{1,2}, \mathrm{C} \mathrm{Palmieri}^{1}, \mathrm{C}_{\text {Parke }}^{1}$, M Mason ${ }^{1}$, P J Blackall ${ }^{2}$, J Botella ${ }^{1}$ and C Turni ${ }^{2}$

${ }^{1}$ The University of Queensland, Australia, ${ }^{2}$ Queensland Alliance for Agriculture and Food Innovation, The Univeristy of Queensland, Australia,

agnes.delacruz@uqconnect.edu.au

Application Point-of-management assays offer the possibility of quicker, cheaper assays for more responsive disease control.

Introduction Detection of pig pathogens is a major challenge. The process includes proper collection of samples and the application of suitable diagnostic methods. Current generation diagnostic tests are typically limited to costly and highly technical laboratory-based assays. A recent study in the Philippines showed that $25 \%$ of small-holder pig farms had pigs with lung lesions at slaughter (Alawneh et al., 2018), emphasising that diseases are important in the small-holder setting. Hence, delivering cost-effective and relevant diagnostic tests suitable for small-holder pig farms is important. Point-ofmanagement or pen-side testing may provide greater access to effective diagnostic services for both small-holder and commercial pig farms. This study validated and optimised the capacity of novel cellulose dipstick method to extract DNA from oral fluid samples, using porcine circovirus type 2 (PCV2) as a model pathogen, as part of our development of a pen-side testing workflow.

Materials and methods Oral fluid (OF) samples were collected from 6 week old pigs ( $\sim 15$ to 20 pigs per pen) by placing cotton ropes inside the pen of pigs (Prickett et al., 2008). These samples were used to validate and optimise a recently described novel cellulose dipstick extraction method (Zou et al., 2017). The efficacy of this method was measured by spiking the OF, as well as phosphate buffered saline (PBS) as a control, with dilutions of known concentrations of PCV2 artificial target DNA (from $1.13 \times 10^{10}$ to $10^{4}$ copies of PCV2 DNA per ml). To overcome possible inhibitors in the OF, the OF was diluted 1:100 before spiking. The samples were examined using a quantitative real-time polymerase chain reaction (qPCR) (Olvera et al., 2004). The dipstick extraction method was compared with a commercial extraction kit (Qiagen ${ }^{\circledR}$ DNeasy blood and tissue kit, Qiagen, Chadstone, VIC) as the standard method.

Results The novel cellulose dipstick method was capable of extracting DNA from PCV2-spiked OF samples in comparison with the commercial kit (Table 1). The results showed almost the same amplification values in both extraction methods except that the dipstick extraction method was able to detect down to $1.65 \times 10^{4}$ copies per ml compared to kit extraction method that can detect $5.6 \times 10^{4}$ copies per $\mathrm{ml}$ of spiked diluted OF. As well, the validation tests using qPCR showed almost the same amplification values with the spiked diluted OF and spiked PBS.

Table 1. Amplification values of PCV2-spiked PBS and oral fluid using either a commercial kit or a novel dipstick extraction method

\begin{tabular}{|c|c|c|c|c|c|c|c|c|}
\hline \multirow[b]{2}{*}{$\begin{array}{l}\text { Extraction } \\
\text { method }\end{array}$} & \multirow[b]{2}{*}{ Medium* } & \multicolumn{7}{|c|}{ Calculated PCV2 level for the indicated levels of spiked PCV2 DNA } \\
\hline & & $\begin{array}{l}1.13 \times 10^{10} \\
\text { copies/ml }\end{array}$ & $\begin{array}{l}1.13 \times 10^{9} \\
\text { copies } / \mathrm{ml}\end{array}$ & $\begin{array}{c}1.13 \times 10^{8} \\
\text { copies/ml }\end{array}$ & $\begin{array}{l}1.13 \times 10^{7} \\
\text { copies/ml }\end{array}$ & $\begin{array}{l}1.13 \times 10^{6} \\
\text { copies } / \mathrm{ml}\end{array}$ & $\begin{array}{l}1.13 \times 10^{5} \\
\text { copies } / \mathrm{ml}\end{array}$ & $\begin{array}{l}1.13 \times 10^{4} \\
\text { copies/ml }\end{array}$ \\
\hline Kit & PBS & $4.90 \times 10^{9}$ & $5.30 \times 10^{8}$ & $6.78 \times 10^{7}$ & $6.68 \times 10^{6}$ & $6.28 \times 10^{5}$ & $2.10 \times 10^{5}$ & $4.76 \times 10^{4}$ \\
\hline Dipstick & PBS & $2.60 \times 10^{9}$ & $1.18 \times 10^{8}$ & $2.80 \times 10^{7}$ & $4.90 \times 10^{6}$ & $3.76 \times 10^{5}$ & $3.80 \times 10^{4}$ & $4.52 \times 10^{4}$ \\
\hline Kit & OF & $2.62 \times 10^{9}$ & $3.54 \times 10^{8}$ & $2.96 \times 10^{7}$ & $3.84 \times 10^{6}$ & $4.48 \times 10^{5}$ & $5.60 \times 10^{4}$ & 0 \\
\hline Dipstick & $\mathrm{OF}$ & $2.14 \times 10^{9}$ & $2.14 \times 10^{8}$ & $3.14 \times 10^{7}$ & $2.40 \times 10^{6}$ & $3.00 \times 10^{5}$ & $3.42 \times 10^{4}$ & $1.65 \times 10^{4}$ \\
\hline
\end{tabular}

*Medium, in which the spiked DNA was resuspended; PBS = phosphate buffered saline; OF = oral fluid

Conclusions In this work, the use of novel cellulose dipstick was shown to be a simple way to extract viral DNA using easily collected OF as the sample. This confirms the potential use of OF as a sample for detection of pig pathogens within a pig herd. The use of an OF sample coupled with an easy extraction method and portable amplification method may open avenues that will allow more rapid detection of pathogens than the current methods of centralised highly equipped laboratories. These tests could readily be applied on both commercial and small-holder farms and would allow more targeted interventions and better production outcomes. Further work is in progress in optimisation of the assay to use in the farm setting.

Acknowledgements Supported in part by the Science with Impact Fund (The University of Queensland) and ACIAR John Allwright Fellowship.

\section{References}

Alawneh J, Parke C, Lapuz EJ, David JE, Basinang V, Baluyut A, Barnes T, Villar E, Lopez M, Meers J and Blackall PJ 2018. Frontiers in Veterinary Science. 5, 1-9.

Prickett J, Kim W, Simer R, Yoon KJ and Zimmerman J 2008. Journal of Swine Health and Production. 16, 86-91. Olvera A, Sibila M, Calsamiglia M, Segalés J and Domingo M 2004. Journal of Virological Methods. 117, 75-80. Zou Y, Mason M, Wang Y, Wee E, Turni C, Blackall PJ, Trau M and Botella JR 2017. PLOS Biology. 15, 1-22. 


\section{Prevalence of MRSA in Australian pig herds and molecular typing of isolates}

$\underline{\text { S Sahibzada }}^{1,2,4}$, M Hernández-Jover ${ }^{1,2}$, D Jordan $^{3}, \mathrm{~S}_{\text {Abraham }}^{4}$, M O'Dea ${ }^{4}$ and J Heller ${ }^{1,2}$

${ }^{1}$ Charles Sturt University, Australia, ${ }^{2}$ Graham Centre for Agricultural Innovation, Australia, ${ }^{3}$ New South Wales Department of Primary Industries, Australia, ${ }^{4}$ Murdoch University, Australia

sshafi@murdoch.edu.au

Application This study advances the broader understanding of Methicillin-resistant Staphylococcus aureus (MRSA) in pigs in Australia by describing the prevalence of carriage and molecular characterisation. Finding ciprofloxacin resistance among MRSA isolates in the absence of fluoroquinolone use indicates the introduction of resistant clone and suggests that improved biosecurity is a prime tool to reduce the spread of resistance in piggeries.

Introduction Livestock-associated (LA) MRSA has been reported as an occupational health issue for piggery workers across the globe (Crombé et al., 2013; Liu et al., 2015). Recently, a high prevalence of community-associated (CA) MRSA was found on a single commercial piggery in Australia (Sahibzada et al., 2017) which raised concerns that the molecular epidemiology of MRSA in pigs might be different in Australia compared to the rest of the world (EFSA and ECDC 2014). This study aimed to measure the presence and distribution of MRSA prevalence among pigs in commercial piggeries in Australia with a focus on identifying the sequence types present and investigate patterns of antibiotic resistance and use amongst the participating piggeries.

Materials and methods A cross-sectional study was performed on 26 commercial pig farms distributed throughout Australia during January-October 2017. Five environmental and 60 nasal swabs from weaners were collected from each farm. MALDI-TOF was performed on all isolates for the $S$. aureus species identification. Presumptive MRSA isolates were typed using real-time PCR and phenotypically assessed for resistance to 12 different antibiotics.

Results MRSA was identified in 53.9\% (14/26) of the study farms, and animal-level prevalence estimates ranged between $1.6 \%$ and $100 \%$ on positive farms. On average, the prevalence of MRSA carriage in pigs on MRSA positive farms was $40.28 \%$ (95\% CI 27.0-53.5\%). All MRSA isolates were typed as ST398, and lacked the lukF-lukS genes encoding PantonValentine leucocidin toxin. All isolates were resistant to tetracycline. A high proportion of isolates were resistant to clindamycin (94.6\%), erythromycin (76.7\%), and amoxicillin-clavulanate $(68.3 \%)$. Resistance was also noted for chloramphenicol (30.5\%) and quinupristin-dalfopristin (17.2\%), gentamicin (4.1\%), neomycin (4.1\%), and sulfamethoxazole-trimethoprim (1.6\%). One-quarter of MRSA isolates were resistant to ciprofloxacin (25.8\%). The most commonly reported antibiotics used among the piggeries were: amoxicillin (used in $92.3 \%$ of the piggeries sampled), penicillin $(92.3 \%)$, tetracycline $(80.8 \%)$, tylosin $(80.8 \%)$, sulfamethoxazole-trimethoprim (69.2\%), lincomycin (53.8\%), and ceftiofur $(30.8 \%)$.

Conclusion This study confirms that LA-MRSA ST398 was the predominant strain circulating in the sampled herds. Highly pathogenic CA-MRSA ST93 was present at low levels with carriage detected only on a single farm (Sahibzada et al., 2017). Demonstration of ciprofloxacin resistance was noteworthy given that the use of fluoroquinolones is not permitted in food animals in Australia; therefore, it is unlikely to have evolved as a result of local selection pressure within pig herds. The information collected by questionnaire revealed that biosecurity and hygiene practices at the farm level could be further improved, and this may also be important in the management of fluoroquinolone resistance.

Acknowledgements Supported in part by Australian Pork Limited. The authors would like to acknowledge the participation of producers in this study.

\section{References}

Crombé F, Argudín MA, Vanderhaeghen W, Hermans K, Haesebrouck F and Butaye P 2013. Frontiers in Microbiology. 4, 1-21.

EFSA and ECDC 2014. The European Union summary report on antimicrobial resistance in zoonotic and indicator bacteria from humans, animals and food in 2012. EFSA Journal. 12, 1-336.

Liu W, Liu Z, Yao Z, Fan Y, Ye X and Chen S 2015. American Journal of Infection Control. 43, 469-475.

Sahibzada S, Abraham S, Coombs GW, Pang S, Hernández-Jover M, Jordan D and Heller J 2017. Scientific Reports. 7, $1-11$. 
Coarse milled wheat inclusion in a pelleted feed provides no health benefits and reduces feed efficiency in growing, but not finishing pigs

C J Brewster $^{1}$, E Klapish ${ }^{1}$ and D J Henman ${ }^{1}$

${ }^{1}$ Rivalea Australia Pty Ltd, Australia

cbrewster@rivalea.com.au

Application Coarsely milled wheat can be used at $150 \mathrm{~kg} / \mathrm{t}$ in finisher pig (60 to $105 \mathrm{~kg}$ ) diets without negatively impacting feed conversion efficiency. However, in grower pigs (35 to $60 \mathrm{~kg}$ ) addition of coarsely milled wheat at 150 $\mathrm{kg} / \mathrm{t}$ can negatively impact feed conversion efficiency. There did not appear to be any benefits to pig enteric health from feeding coarsely milled wheat.

Introduction Addition of whole or coarsely milled grains is common in commercial broiler chicken feeding to improve enteric health and increase feed mill throughput and efficiency (e.g. Ravindran et al., 2006). While much of the benefit in broilers is attributed to gizzard health, Brunsgaard (1998) suggested that coarsely milled grains can promote gut health and short-chain fatty acid (SCFA) production from fermentation of undigested starch in the hind-gut of grower/finisher pigs. Our hypothesis was that addition of $150 \mathrm{~kg} / \mathrm{t}$ of coarsely milled wheat added either within a pellet or mixed externally to a pellet would improve health of grower-finisher pigs without negatively impacting growth performance.

Material and methods One thousand and fifty-six mixed sex Large White x Landrace (Primegro ${ }^{\mathrm{TM}}$ Genetics, Corowa, NSW) pigs, housed in 24 pens of 44 pigs/pen were randomly allocated among three dietary treatments at 12 weeks of age $(35.3 \pm 0.3 \mathrm{~kg})$. The control diet (FW) was manufactured using finely milled wheat (median particle size $700 \mu \mathrm{m})$ as the main ingredient. Two other dietary treatments replaced $150 \mathrm{~kg} / \mathrm{t}$ of fine milled wheat with a coarsely milled wheat (median particle size $1400 \mu \mathrm{m}$ ). The coarse wheat was either incorporated internally within the pellet (CWIP) or added post pelleting (CWPP). Grower diets were formulated to $14 \mathrm{MJ}$ digestible energy (DE)/kg and $0.70 \mathrm{~g}$ standard ileal digestible (SID) lysine/MJ DE and finisher diets to $13.5 \mathrm{MJ} \mathrm{DE} / \mathrm{kg}$ and $0.60 \mathrm{~g}$ SID lysine/MJ DE. Feed and water were available $a d$ libitum. Pig weights and feed intakes were measured at 16 weeks and 22 weeks of age after which they were commercially slaughtered and carcass data (carcass weight, P2 loin and fat depth) were collected. Growth and carcass data were analysed as a 2 x 3 (sex x treatment) factorial ANOVA. All pig mortalities were recorded and tested for significance via chi-square analysis (IBM SPSS Statistics for Windows, v24.0. Armonk, NY).

Results In the grower period pigs fed pellets made with fine ground wheat ate less and grew more efficiently than pigs fed $150 \mathrm{~kg} / \mathrm{t}$ coarse wheat added internally or post-pelleting $(P<0.05$, Table 1$)$. However, in the finisher period $150 \mathrm{~kg} / \mathrm{t}$ of coarse wheat can be added to feed without negative impact on feed efficiency. There was no effect of dietary treatment on mortalities, or on any carcass measurements.

Table 1. Average daily gain (ADG), feed conversion ratio (FCR) and average daily feed intake (ADFI) of grower (1216 weeks) and finisher (17-22 weeks of age) pigs fed pellets with fine wheat (FW) or coarse wheat added internally (CWIP) or post-pelleting (CWPP)

\begin{tabular}{lcccccc}
\hline \multicolumn{7}{c}{ Frower } \\
& $\begin{array}{c}\text { ADG } \\
(\mathrm{kg} / \mathrm{d})\end{array}$ & $\begin{array}{c}\text { FCR } \\
(\mathrm{kg} / \mathrm{kg})\end{array}$ & $\begin{array}{c}\text { ADFI } \\
(\mathrm{kg} / \mathrm{d})\end{array}$ & $\begin{array}{c}\text { ADG } \\
(\mathrm{kg} / \mathrm{d})\end{array}$ & $\begin{array}{c}\text { FCR } \\
(\mathrm{kg} / \mathrm{kg})\end{array}$ & $\begin{array}{c}\text { ADFI } \\
(\mathrm{kg} / \mathrm{d})\end{array}$ \\
\hline FW & 0.822 & $1.91^{\mathrm{a}}$ & $1.557^{\mathrm{a}}$ & 1.044 & 2.48 & 2.589 \\
CWIP & 0.829 & $2.01^{\mathrm{b}}$ & $1.660^{\mathrm{b}}$ & 1.028 & 2.50 & 2.565 \\
CWPP & 0.835 & $2.04^{\mathrm{b}}$ & $1.695^{\mathrm{b}}$ & 1.037 & 2.55 & 2.643 \\
SEM & 0.012 & 0.03 & 0.021 & 0.016 & 0.03 & 0.036 \\
$P$ value & $\mathrm{NS}$ & 0.01 & 0.001 & NS & NS & NS \\
\hline
\end{tabular}

${ }^{\mathrm{ab}}$ Means within a column with different superscripts differ significantly $(P<0.05)$. SEM, standard error of mean

Conclusion The hypothesis was rejected. There was no improvement in pig mortality from addition of $150 \mathrm{~kg} / \mathrm{t}$ of coarsely milled wheat to grower or finisher diets. However, further research measuring gut histology and/or SCFA levels along to gastro-intestinal tract is required. Furthermore, grower pigs exhibited an increased feed usage and poorer feed conversion when coarse wheat was used. In contrast, finisher pigs can effectively use up to $150 \mathrm{~kg} / \mathrm{t}$ of coarsely milled wheat added internally or post-pelleting. Use of coarsely milled wheat added post-pelleting can increase feed mill throughput and reduce manufacturing costs; however, this should only be considered in finishing pigs where feed efficiency is not likely to be negatively impacted.

\section{References}

Ravindran R, Wu YB, Thomas DG and Morel PC 2006. Australian Journal of Agricultural Research. 57, 21-26. Brunsgaard G 1998. Journal of Animal Science. 11, 2787-2798. 


\title{
Effect of standardized ileal digestible lysine and added copper on growth performance, nutrient digestibility and backfat thickness in growing pigs
}

\author{
Y M Kim ${ }^{1}, \mathrm{X} \mathrm{Ao}^{1}, \mathrm{~J} \mathrm{Hu}^{1}, \mathrm{~S} \mathrm{Zhang}^{1}$ and $\underline{\mathrm{H} \mathrm{Kim}}^{1}$ \\ ${ }^{I}$ Department of Animal Resource \& Science, Dankook University, Republic of Korea \\ inhokim@dankook.ac.kr
}

Application This study showed the effect of increasing standardized ileal digestible lysine with copper in growingfinishing pigs, which indicate some positive effects with increasing lysine levels.

Introduction Coble et al. (2017) found that diets with reduced standardized ileal digestible lysine (SID Lys) $0.05 \%$ below the requirement of NRC (2012) and $150 \mathrm{mg} / \mathrm{kg}$ added copper $(\mathrm{Cu})$ increased ADFI and $\mathrm{G}: \mathrm{F}$ in finishing pigs (over 88 $\mathrm{kg}$ ). In this study our aim was to determine if the increase seen by Coble et al. (2017) is improved when levels of SID Lys above the requirement of NRC are fed in combination with $150 \mathrm{mg} / \mathrm{kg}$ added $\mathrm{Cu}$ in growing pigs.

Material and methods Two hundred and forty castrated male pigs [(Landrace $\mathrm{x}$ Yorkshire) $\mathrm{x}$ Duroc] with an average initial body weight (BW) of $22.7 \pm 0.11 \mathrm{~kg}$ were randomly assigned to 1 of 4 corn-soy based dietary treatments: $100 \%$ of estimated SID Lys requirement based on NRC (2012) modelling, with $0 \mathrm{mg} / \mathrm{kg}$ additional $\mathrm{Cu}$ (above nutritional requirements), and 100, 103, or $106 \%$ of the estimated SID Lys requirement with $150 \mathrm{mg} / \mathrm{kg}$ added Cu, from copper sulfate. With all other nutrients meeting NRC (2012) requirements. There were 10 pens per treatment and 6 pigs per pen. Individual pig BW was measured on $\mathrm{d} 1$ and 42 of the experiment. Feed consumption per pen was also assessed on $\mathrm{d} 42$ of the experiment. The average daily gain (ADG), average daily feed intake (ADFI), and G:F across the experiment were calculated. During d 35-42, chromic oxide ( $0.2 \%)$ was added to all the diets as an indigestible marker for the determination of apparent nutrient digestibility of dry matter (DM), nitrogen (N) and gross energy (GE). All data were subjected to the GLM procedures of SAS (SAS Institute Inc.; Cary, NC, USA) as a randomized complete block design with the pen being considered as the experimental unit. A probability level of $P<0.05$ was considered to be statistically significant.

Results The final BW, ADG and estimated SID Lys intake in pigs fed added Cu diets with 103 and 106\% SID Lys were increased $(P<0.05)$ compared with those fed CON diet. The final BW, ADG, ADFI, G:F and estimated SID Lys intake in added $\mathrm{Cu}$ treatments increased $(P<0.05)$ linearly with increasing SID Lys levels. Within the Cu groups, the apparent digestibility of DM, N and GE increased linearly $(P<0.05)$ in response to increasing SID Lys level (not shown), as did backfat thickness $(P<0.05)$.

Table 1. Effects of increased levels of standardized ileal digestible lysine (SID Lys) and supplemental copper on growth performance (BW, body weight; ADG, average daily gain; ADFI, average daily feed intake; G:F, gain:feed

\begin{tabular}{|c|c|c|c|c|c|c|c|c|}
\hline \multirow{2}{*}{$\begin{array}{l}\text { Copper }(\mathrm{mg} / \mathrm{kg}) \\
\text { SID Lys, }(\% \text { of NRC) }\end{array}$} & 0 & 150 & 150 & 150 & \multirow{2}{*}{ SEM } & \multicolumn{3}{|c|}{$P$ value } \\
\hline & 100 & 100 & 103 & 106 & & Contrast 1 & Contrast 2 & Linear \\
\hline Initial BW (kg) & 22.8 & 22.8 & 22.7 & 22.7 & 0.11 & 0.61 & 0.53 & 0.97 \\
\hline $\begin{array}{l}\text { Final BW }(\mathrm{kg}) \\
\text { d } 0-42\end{array}$ & 53.1 & 53.7 & 54.4 & 55.0 & 0.52 & 0.34 & 0.04 & 0.03 \\
\hline $\operatorname{ADG}(\mathrm{kg} / \mathrm{d})$ & 0.72 & 0.74 & 0.76 & 0.77 & 0.01 & 0.37 & 0.03 & 0.02 \\
\hline ADFI, $(\mathrm{kg} / \mathrm{d})$ & 1.64 & 1.66 & 1.69 & 1.70 & 0.02 & 0.40 & 0.23 & 0.04 \\
\hline $\mathrm{G}: \mathrm{F}(\mathrm{kg} / \mathrm{kg})$ & 0.441 & 0.442 & 0.446 & 0.452 & 0.005 & 0.56 & 0.73 & 0.01 \\
\hline Estimated SID lysine (g/d) & 16.1 & 16.2 & 17.1 & 17.5 & 0.05 & 0.62 & 0.02 & 0.01 \\
\hline
\end{tabular}

SEM, pooled standard error of the means; Contrast 1, Control vs $150 \mathrm{mg} / \mathrm{kg} \mathrm{Cu}$ with 100\% SID Lys; Contrast 2, Control vs $150 \mathrm{mg} / \mathrm{kg} \mathrm{Cu}$ with 103\% SID lysine and 106\% SID lysine of NRC; Linear, $150 \mathrm{mg} / \mathrm{kg} \mathrm{Cu}$ with 100, 103 and $106 \%$ SID Lys.

Conclusion Adding supplemental $\mathrm{Cu}$ in the form of copper sulfate did not increase nutrient digestibility and backfat thickness, compared with increasing the SID Lys which lead to an increase in growth performance in growing pigs from 22 to $53 \mathrm{~kg}$, linear response in the presence of $\mathrm{Cu}$.

\section{References}

Coble KF, DeRouchey JM, Tokach MD, Dritz SS, Goodband RD, Woodworth JC and Usry JL 2017. Journal of Animal Science. 95, 4052-4059. 
Feeding Lupinus albus $L$. to immunocastrated male pigs to reduce feed intake and backfat $\underline{\mathrm{K} \mathrm{L} \mathrm{Moore}}^{1,2}$, E Loudon $^{2}$ and F R Dunshea ${ }^{3}$

${ }^{1}$ Pork Innovation WA Inc., Australia, ${ }^{2}$ KLM Consulting, Australia, ${ }^{3}$ University of Melbourne, Australia karen@klmconsulting.com.au

Application Albus lupins can be included in the diet of immunocastrated male pigs to decrease feed intake. The concentration of albus lupins to include in the diet will depend on which parameter is desired to be optimized.

Introduction Immunisation against gonadotropin-releasing factor (Improvac ${ }^{\circledR}$, Zoetis Inc., Rhodes, NSW) is an effective strategy to eliminate boar taint. However, immunocastrated (IC) males have an increased feed intake, growth rate and backfat compared to entire males (Dunshea et al., 2001a). Lupinus albus L. (albus lupins) have been found to reduce feed intake in pigs with the most likely mechanism being delayed transit through the stomach and small intestine which then feeds back on satiety signals (Dunshea et al., 2001b). The inclusion of albus lupins in diets has shown potential in overcoming the issues of increased feed intake and backfat associated with IC males but the optimal inclusion concentration of albus lupins in diets to maximise the decrease in feed intake and backfat whilst minimising the negative effect on growth performance needs to be determined (Moore et al., 2017). The aim of this experiment was to determine the concentration of albus lupins to be included in the diet of IC males which would maximise the decrease in feed intake and backfat whilst optimising the effect on growth rate.

Material and methods Two hundred and sixteen (Large White $x$ Landrace $x$ Duroc) IC male pigs were used in a completely randomised experiment with six concentrations of albus lupins $(0,40,80,120,160$ and $200 \mathrm{~g} / \mathrm{kg})$. The diets were fed from two weeks after the second immunization against GnRF for $14 \mathrm{~d}$ pre-slaughter. The diets were formulated to be 13.5 MJ digestible energy (DE) and $0.50 \mathrm{~g}$ standard ileal digestible lysine/MJ DE. Pigs were $96.0 \pm 0.60 \mathrm{~kg}$ (mean $\pm \mathrm{SE})$ at the commencement of the experiment and $112.4 \pm 0.99 \mathrm{~kg}$ at slaughter. Pigs were housed in groups of 6 and there were six replicates/treatment. All pigs had ad libitum access to water and feed via a single-spaced feeder. Pigs were weighed and feed disappearance was recorded weekly and carcass characteristcs were recorded at slaughter. One way analysis of variance was performed (GenStat 18th ed, VSN International, Hemel Hempstead, UK). The optimal concentration of albus lupins to include in the diet was identified for each significantly different parameter using a split line regression fitted to the treatment means (GenStat 18th ed, VSN International, Hemel Hempstead, UK).

Results Increasing the concentration of albus lupins decreased final liveweight $(P=0.005$, data not shown), average daily gain $(P=0.004)$ and feed intake $(P<0.001)$ with no effect on the feed conversion ratio $(P>0.05$, data not shown). Carcass weight $(P=0.011)$ and backfat $(P=0.021)$ decreased as the concentration of albus lupins in the diet increased. The predicted concentration of albus lupins in the diet to optimise daily gain, feed intake, carcass weight and backfat was $120,142,62.7$ and $132 \mathrm{~g} / \mathrm{kg}$, respectively.

Table 1. Growth performance and carcass characteristics for immunocastrated male pigs fed different concentrations of albus lupins from 78.9 to $112.4 \mathrm{~kg} \mathrm{LW}$ ( $\mathrm{n}=6$ groups of 6 pigs per treatment).

\begin{tabular}{|c|c|c|c|c|c|c|c|c|c|}
\hline & \multicolumn{6}{|c|}{ Albus lupins $(\mathrm{g} / \mathrm{kg})$} & \multirow[b]{2}{*}{ SED } & \multirow[b]{2}{*}{$P$ value } & \multirow{2}{*}{$\begin{array}{c}\text { Predicted } \\
\text { conc. }(\mathrm{g} / \mathrm{kg})\end{array}$} \\
\hline & 0 & 40 & 80 & 120 & 160 & 200 & & & \\
\hline Daily gain (kg/day) & $1.33^{\mathrm{a}}$ & $1.22^{\mathrm{ab}}$ & $1.16^{\mathrm{bc}}$ & $1.09^{\mathrm{bc}}$ & $1.19^{\mathrm{ab}}$ & $1.03^{\mathrm{c}}$ & 0.072 & 0.004 & 120 \\
\hline Feed intake ( $\mathrm{kg}$ /day) & $4.04^{\mathrm{a}}$ & $4.05^{\mathrm{a}}$ & $3.74^{\mathrm{ab}}$ & $3.62^{\mathrm{b}}$ & $3.59^{\mathrm{b}}$ & $3.45^{\mathrm{b}}$ & 0.178 & 0.012 & 142 \\
\hline Carcass weight (kg) & $74.8^{\mathrm{a}}$ & $74.9^{\mathrm{a}}$ & $74.8^{a}$ & $72.6^{\mathrm{bc}}$ & $73.8^{a b}$ & $71.5^{\mathrm{c}}$ & 1.043 & 0.011 & 62.7 \\
\hline Backfat depth (mm) & $12.2^{\mathrm{a}}$ & $11.6^{\mathrm{ab}}$ & $11.8^{\mathrm{ab}}$ & $11.0^{\mathrm{bc}}$ & $11.7^{\mathrm{ab}}$ & $10.2^{\mathrm{c}}$ & 0.563 & 0.021 & 138 \\
\hline
\end{tabular}

${ }^{\mathrm{a}, \mathrm{b}, \mathrm{c}}$ Means within a row with different superscripts differ significantly $(P<0.05)$; SED, standard error of difference of the means; ${ }^{2}$ Backfat measured at P2 site $65 \mathrm{~mm}$ from the midline at the head of the last rib.

Conclusion Albus lupins can be included in the diets of immunocastrated male pigs for $14 \mathrm{~d}$ pre-slaughter (two weeks after the second immunization against GnRF) to decrease feed intake and subsequently backfat. However, as the concentration of albus lupins increases the growth rate decreases. The concentration of albus lupins to include in the diet will ultimately depend on which parameter is desired to be optimized by the producer.

Acknowledgements Supported by the Agricultural Produce Commission - Pork Producers Committee.

\section{References}

Dunshea FR, Calantoni C, Howard K, McCauley I and Jackson P 2001a. Journal of Animal Science. 79, $2524-2535$.

Dunshea FR, Gannon NJ, van Barneveld RJ, Mullan BP, Campbell RG and King RH 2001b. Australian Journal of Agricultural Research. 52, 593-602.

Moore KL, Mullan BP, Kim JC and Dunshea FR 2017. Animals. 7, 15. 


\section{Disappearance of dietary fibre in the small intestine, large intestine, and total tract of growing pigs fed corn- or wheat-based diets without or with microbial xylanase}

$\mathrm{J}_{\mathrm{J}}$ Abelilla ${ }^{1,2}$ and $\mathrm{H} \mathrm{H}_{\text {Stein }}{ }^{1}$

${ }^{1}$ University of Illinois at Urbana-Champaign, USA, ${ }^{2}$ DSM Nutritional Products, Singapore

hstein@illinois.edu

Application Microbial xylanase supplementation may improve disappearance of energy in corn-based diets and may improve disappearance of energy and dietary fibre in wheat-based diets. This indicates differences in fibre structure in wheat compared with corn and that microbial xylanase may improve energy utilization in growing pigs.

Introduction Disappearance of dietary fibre along the intestinal tract is influenced by the structure and physical characteristics of fibre (Bach Knudsen, 2001). The objective of the experiment was to test the null hypothesis that there is no difference in the disappearance of nutrients and dietary fibre in the small intestine, large intestine, and total tract of pigs fed diets based on corn and soybean meal (SBM), corn, SBM, and corn distillers dried grains with solubles (DDGS), wheat and SBM, or wheat, SBM, and wheat middlings without or with microbial xylanase.

Material and methods Diets based on corn and SBM; or corn, SBM, and 30\% DDGS; wheat and SBM; or wheat, SBM and $30 \%$ wheat middlings were formulated without xylanase, with Xylanase A, or with Xylanase B for a total of 12 diets. Diets were randomly allotted to 24 barrows (initial BW: $28.51 \pm 1.86 \mathrm{~kg}$ ) following a $24 \mathrm{x} 4$ Youden square design with 12 diets and four 18-d periods, for a total of 8 replicate pigs per diet. Pigs had a T-cannula installed in the duodenum and a second T-cannula was placed in the ileum. Each period lasted $18 \mathrm{~d}$. The initial $7 \mathrm{~d}$ was an adaptation period to the diets. Faeces and urine were collected from the feed provided from d 8 to 13. Ileal digesta were collected on d 15 and 16, and duodenal digesta were collected on d 17 and 18 (González-Vega et al., 2014). Diets, duodenal and ileal digesta, and faeces samples were analyzed for nutrients, energy, insoluble dietary fibre, and soluble dietary fibre. Urine samples were analyzed for energy and disappearance of nutrients, energy, and total dietary fibre (TDF) was calculated. Data were analyzed following a $2 \times 2 \times 3$ design with 2 types of diets (corn-based or wheat-based), 2 levels of fibre (low or high), and 3 microbial xylanase treatments (none, Xylanase A, or Xylanase B) using the MIXED procedure (SAS Institute Inc., Cary, NC, USA) with pig as the experimental unit.

Results Disappearance of gross energy (GE) in the small intestine and large intestine of pigs was 12.88 and $3.20 \mathrm{MJ} / \mathrm{kg}$ DMI, respectively, and disappearance of TDF in the small intestine and large intestine was $32 \mathrm{and} 72 \mathrm{~g} / \mathrm{kg} \mathrm{DMI}$, respectively. Disappearance of GE in the small intestine was improved $(P<0.05)$ with inclusion of Xylanase B in cornSBM diets (14.50 vs $12.89 \mathrm{MJ} / \mathrm{kg}$ DMI). Disappearance of GE and TDF from the wheat-SBM-wheat middlings diet in the large intestine was improved $(P<0.05)$ if Xylanase B was included (4.13 vs $3.47 \mathrm{MJ} / \mathrm{kg}$ DMI and 96 ss $76 \mathrm{~g} / \mathrm{kg}$ DMI). Total tract disappearance of GE and TDF was improved $(P<0.05)$ in the wheat-SBM diet if Xylanase A was used $(17.18$ vs $16.87 \mathrm{MJ} / \mathrm{kg}$ and 121 vs $114 \mathrm{~g} / \mathrm{kg}$ DMI) and in wheat-SBM-wheat middlings diets if Xylanase A or B was used (16.29 or $16.23 \mathrm{MJ} / \mathrm{kg}$ vs 15.92 and 182 or 184 vs $173 \mathrm{~g} / \mathrm{kg} \mathrm{DMI})$. Total tract disappearance of TDF in wheat-based diets was reduced $(P<0.05)$ compared with corn-based diets $(149$ vs $116 \mathrm{~g} / \mathrm{kg} \mathrm{DMI})$.

Conclusion Disappearance of energy in corn- and wheat-based diets occurs mainly in the small intestine and disappearance of dietary fibre occurs mainly in the large intestine of pigs. Disappearance of energy in the small intestine in corn-based diets may be improved if microbial xylanase is supplemented, but this was not the case for disappearance of dietary fibre. Disappearance of energy and dietary fibre in the large intestine and total tract may be improved if microbial xylanase is supplemented to wheat-based diets. Xylanase B appears to be more efficient than Xylanase A in improving disappearance of energy and dietary fibre both in corn- and wheat-based diets indicating variation among xylanase sources. Differences in the total tract disappearance of dietary fibre in corn- vs. wheat-based diets may indicate differences in fibre structure in corn and wheat grains.

Acknowledgements Financial support for this research from Danisco Animal Nutrition-DuPont Industrial Biosciences (Marlborough, UK) and travel support from DSM Nutritional Products are greatly appreciated.

\section{References}

Bach Knudsen KE 2001. Animal Feed Science and Technology. 90, 3-20.

González-Vega JC, Walk CL, Liu Y and Stein HH 2014. Archives of Animal Nutrition. 68, 126-142. 


\section{Isoquinoline alkaloids and betaine supplementation ameliorate heat stress in grower pigs}

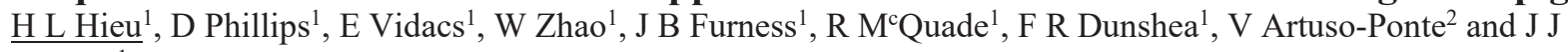

Cottrell $^{1}$

${ }^{I}$ The University of Melbourne, Australia. ${ }^{2}$ Phytobiotics Futterzusatzstoffe GmbH, Germany.

huul1@student.unimelb.edu.au

Application The feed additives Sangrovit Extra and betaine are useful for the management of heat stress in growing pigs.

Introduction Heat stress (HS) compromises efficient pork production in part, due to increased oxidative stress and inflammation, particularly within the gastrointestinal tract (GIT, Cottrell et al., 2015). Recently, there has been an increasing focus on the use of nutritional supplements as cost effective tools for the amelioration of HS. The effect of isoquinoline alkaloid extracted from Macleaya cordata (Sangrovit Extra, Phytobiotics GmbH, Eltville, Germany) on symptoms of HS and GIT integrity were studied (Artuso-Ponte et al., 2015). Sugar beet derived betaine is both a methyl donor and osmolyte that reduces oxidative stress, preventing tissues damage and inflammation (Gabler et al., 2013). Thus, the aim of this experiment was to investigate if the supplementation of Sangrovit Extra (SAN) and betaine ameliorate thermoregulatory responses to HS and protect intestinal integrity in HS grower pigs.

Material and methods Fifty female Large White x Landrace grower pigs (av. $27.3 \mathrm{~kg}$ ) were randomly acclimated to one of three experimental diets: control ( $\mathrm{CON}, \mathrm{n}=18$, standard grower diet), betaine (BET, $+1 \mathrm{~g} / \mathrm{kg}$ betaine, $\mathrm{n}=16)$, SAN $(+0.15 \mathrm{~g} / \mathrm{kg})$ for $14 \mathrm{~d}$ under thermoneutral conditions $\left(\mathrm{TN}\right.$, constant $\left.20^{\circ} \mathrm{C}\right)$. After the dietary acclimation, half of the pigs from each diet were then allocated to one of two climate conditions to form a $2 \times 3$ factorial design. The conditions were either TN or cyclic heat-stress conditions (HS; $\left.8 \mathrm{~h} 35^{\circ} \mathrm{C}, 16 \mathrm{~h} 28^{\circ} \mathrm{C} / \mathrm{d}\right)$ for $3 \mathrm{~d}(\mathrm{n}=8-9 \mathrm{trt} / \mathrm{gp})$. Respiration rate (RR), rectal and skin temperature (RT and ST) were measured every $2 \mathrm{~h}$ during the first $2 \mathrm{~d}$ of the HS period. Pigs were euthanised on $\mathrm{d} 3$ of the thermal exposure and blood samples were collected for blood gas and metabolites analyses. Proximal jejunum, distal ileum and colon were harvested for measurement of transepithelial electrical resistance (TER) and fluorescein isothiocyanate-dextran (4 kDa, FD4) permeability with Ussing chambers. All data was analysed using an ANOVA with Duncans post-hoc tests (GenStat $18^{\text {th }}$ ed, VSN International, Hemel Hempstead, UK).

Results Heat stress increased RR, RT and ST compared to TN $(P<0.001)$; however, there was an interaction between diets and temperature in RR $(P=0.013)$ and RT $(P=0.001)$ where both BET and SAN reduced RR and RT compared to CON under heat exposed. Dietary BET improved total antioxidant capacity (TAO) overall $(P=0.009)$. Dietary SAN increased ileal TER under TN, but not in HS conditions $(P=0.05)$. Heat stress increased FD4 permeability in the colon and feeding both BET and SAN reduced the permeability in this site $(P=0.057)$.

Table 1. Physiological responses, antioxidant capacity and intestinal barrier function of pigs fed control, betaine or isoquinoline alkaloid (SAN) under thermoneutral (TN) or heat stress (HS) condition.

\begin{tabular}{|c|c|c|c|c|c|c|c|c|c|c|}
\hline \multirow{2}{*}{ Items } & \multicolumn{3}{|c|}{ TN } & \multicolumn{3}{|c|}{ HS } & \multirow{2}{*}{ SED } & \multicolumn{3}{|c|}{$P$ value } \\
\hline & Con & Betaine & SAN & Con & Betaine & SAN & & Diet & Temp & $\mathrm{D} * \mathrm{~T}$ \\
\hline Respiration rate (breath/mins) & $23^{a}$ & $24^{\mathrm{a}}$ & $22^{\mathrm{a}}$ & $169^{c}$ & $150^{\mathrm{b}}$ & $158^{\mathrm{b}}$ & 5.0 & 0.024 & $<0.001$ & 0.013 \\
\hline Rectal temperature $\left({ }^{\circ} \mathrm{C}\right)$ & $38.5^{\mathrm{a}}$ & $38.6^{\mathrm{a}}$ & $38.6^{\mathrm{a}}$ & $40.1^{\mathrm{c}}$ & $39.8^{\mathrm{b}}$ & $39.9^{\mathrm{b}}$ & 0.07 & 0.40 & $<0.001$ & 0.001 \\
\hline Skin temperature $\left({ }^{\circ} \mathrm{C}\right)$ & $34.6^{\mathrm{a}}$ & $34.8^{\mathrm{ab}}$ & $35.1^{\mathrm{b}}$ & $40.8^{c}$ & $40.1^{\mathrm{c}}$ & $39.9^{c}$ & 0.19 & 0.46 & $<0.001$ & 0.05 \\
\hline TAO (mM Trolox) & 1.32 & 1.63 & 1.17 & 1.35 & 1.65 & 1.21 & 0.19 & 0.009 & 0.77 & 0.99 \\
\hline Ileum TER $\left(\Omega . \mathrm{cm}^{2}\right)$ & $34.8^{\mathrm{a}}$ & $37.2^{\mathrm{a}}$ & $60.8^{\mathrm{b}}$ & $50.3^{\mathrm{ab}}$ & $48.9^{\mathrm{ab}}$ & $47.3^{\mathrm{ab}}$ & 8.87 & 0.15 & 0.36 & 0.05 \\
\hline Colon PER (FD4, $\mu \mathrm{g} / \mathrm{min} . \mathrm{cm})$ & $0.669^{\mathrm{a}}$ & $0.805^{\mathrm{ab}}$ & $0.723^{\mathrm{a}}$ & $1.123^{\mathrm{b}}$ & $0.767^{\mathrm{a}}$ & $0.528^{\mathrm{a}}$ & 0.218 & 0.12 & 0.36 & 0.057 \\
\hline
\end{tabular}

a,b,c Means within a row with different superscripts differ significantly $(P<0.05)$; SED, Standard error of difference of the mean; TER, Transepithelial resistance; PER, Permeability)

Conclusion Sangrovit Extra and betaine supplementation ameliorated parameters of HS and protected against increased colonic permeability in HS grower pigs.

Acknowledgements Supported in part by Phytobiotics Futterzusatzstoffe GmbH, Melbourne Research Scholarship and Australian Pork Limited. Thanks is expressed to Maree Cox, Shannon Holbrook, Fiona Armour and Tracie Helen Storey.

\section{References}

Artuso-Ponte V, Moeller S, Rajala-Schultz P, Medardus JJ, Munyalo J, Lim K and Gebreyes WA 2015. Foodborne Pathogens and Disease. 12, 891-897.

Cottrell JJ, Liu F, Hung AT, DiGiacomo K, Chauhan SS, Leury BJ, Furness JB, Celi P and Dunshea FR 2015. Animal Production Science. 55,1391-1402.

Gabler NK, Frouel S, Awati A, Owusu-Asiedu A, Amerah AM, Patridge GG and Dunshea FR 2013. In Manipulating Pig Production XIV. Australasian Pig Science Association, Australia p. 85. 


\section{Rapid eating-quality assessment of premium Australian pork in an Asian market}

$\underline{\text { E P Bittner }}^{1}$, R J van Barneveld ${ }^{2}$, D N D'Souza ${ }^{2}, \mathrm{H}$ Ashman $^{1}$, S Hutchings ${ }^{1}$ and F R Dunshea ${ }^{1}$

${ }^{I}$ The University of Melbourne, Australia, ${ }^{2}$ SunPork Group, Australia

bittnere@unimelb.edu.au

Application Sensory assessment of Australian pork by native Hong Kong consumers via blind sensory evaluation indicates that perceived bias against the products is not a reflection of its comparative eating-quality.

Introduction While the development of eating quality grading for beef, through the Meat Standards Australia (MSA) grading scheme has underpinned the sensory evaluation of meat samples with untrained consumers (Hwang et al., 2008), comparative industry investment in studies to inform an eating quality model for pork have been low. Furthermore, while qualitative research has shown a general bias against Australian pork quality amongst Asian consumers, few quantitative eating-quality studies have been conducted to establish this as fact and determine why. This research investigated the attitudes and opinions of local Cantonese pork consumers in Hong Kong to several local and Australian pork samples, during sensory sessions which measured consumer's overall liking, acceptability and opinion of different pork products. This study aimed to investigate if this bias existed through blind tasting and evaluate the eating-quality of Australian pork in the Hong Kong market.

Material and methods Cantonese consumers $(n=256)$ were recruited in Hong Kong and offered pork samples of different cuts (loin, scotch and leg) as well as different sources (locally sourced, standard Australian, premium Australian). Locally sourced samples were purchased in Hong Kong from a Welcome supermarket. Standard Australian samples were imported fresh/chilled and reflect the standard and quality seen on Australian supermarket shelves. Australian premium samples were imported fresh/chilled and are from a newly developed premium production line with a focus on eating quality. All samples were served blind without descriptive information. Participants were required to eat and evaluate all samples on a hedonic scale for appearance, aroma, tenderness, juiciness, flavour and overall liking. Finally, consumers were asked to evaluate the sample using check-all-that-apply (CATA) for further descriptive information related to their liking of the sample. All questionnaires were entered into Microsoft Excel and statistics analysed. Hedonic results were analysed using REML (IBM SPSS Statistics for Windows, v25.0. Armonk, NY) with means adjusted for all significant effects.

Results For hedonic assessment, all Australian pork samples scored consistently higher than equivalent local samples across all attributes measured (Table 1, $P \leq 0.001$ ). Between Australian samples, a standard product (Australian standard scotch) had the highest sensory score, but overall the premium product was far more consistent across the entire carcass. There is no data for locally sourced leg as this cut was not available in Hong Kong.

Table 1. Means and pooled standard error (SE) adjusted for significant effects of gender, age, day tested and group number for Cantonese consumers

\begin{tabular}{llcccccc}
\hline & & Appearance & Aroma & Tenderness & Juiciness & Flavour & Overall Liking \\
\hline \multirow{2}{*}{ Loin } & Local & $55.6^{\mathrm{e}}$ & $48.8^{\mathrm{e}}$ & $43.9^{\mathrm{d}}$ & $44.7^{\mathrm{e}}$ & $46.0^{\mathrm{d}}$ & $47.0^{\mathrm{d}}$ \\
& Australian Standard & $56.9^{\mathrm{de}}$ & $52.7^{\mathrm{d}}$ & $44.8^{\mathrm{d}}$ & $45.5^{\mathrm{e}}$ & $50.7^{\mathrm{c}}$ & $49.4^{\mathrm{cd}}$ \\
& Australian Premium & $60.2^{\mathrm{ab}}$ & $58.6^{\mathrm{b}}$ & $53.2^{\mathrm{c}}$ & $54.7^{\mathrm{c}}$ & $58.6^{\mathrm{b}}$ & $57.9^{\mathrm{b}}$ \\
\multirow{5}{*}{ Scotch } & $58.6^{\mathrm{bcd}}$ & $56.6^{\mathrm{bc}}$ & $57.0^{\mathrm{bc}}$ & $57.5^{\mathrm{bc}}$ & $57.8^{\mathrm{b}}$ & $57.6^{\mathrm{b}}$ \\
& Local & $62.8^{\mathrm{a}}$ & $61.8^{\mathrm{a}}$ & $68.1^{\mathrm{a}}$ & $64.4^{\mathrm{a}}$ & $65.2^{\mathrm{a}}$ & $65.1^{\mathrm{a}}$ \\
& Australian Standard & $59.9^{\mathrm{bc}}$ & $56.7^{\mathrm{bc}}$ & $59.4^{\mathrm{b}}$ & $59.0^{\mathrm{b}}$ & $60.0^{\mathrm{b}}$ & $60.5^{\mathrm{b}}$ \\
& Australian Premium & $56.2^{\mathrm{de}}$ & $52.5^{\mathrm{d}}$ & $43.9^{\mathrm{d}}$ & $46.4^{\mathrm{e}}$ & $50.3^{\mathrm{c}}$ & $50.3^{\mathrm{c}}$ \\
& Australian Standard & $57.6^{\mathrm{cde}}$ & $54.2^{\mathrm{cd}}$ & $47.6^{\mathrm{d}}$ & $49.8^{\mathrm{d}}$ & $51.8^{\mathrm{c}}$ & $51.6^{\mathrm{c}}$ \\
& Australian Premium & 1.12 & 1.25 & 2.00 & 1.69 & 1.40 & 1.36 \\
& SE & $<0.001$ & $<0.001$ & $<0.001$ & $<0.001$ & $<0.001$ & $<0.001$ \\
\hline
\end{tabular}
a,b,c,d,e Means within a column with different superscripts differ significantly $(P<0.05)$

Conclusion Results clearly indicate that any bias against Australian products in the Hong Kong market are not due to sensory aspects of products or quality, even for standard Australian products. The higher overall consistency of the premium pork across the entire carcass is promising for the Asian market where different cooking methods require versatile, consistent product without dominating meat flavours.

Acknowledgements Supported in part by the Queensland Government 'Growing Queensland Exports Grant'.

\section{References}

Hwang IH, Polkinghorn R, Lee JM and Thompson JM 2008. Australian Journal of Experimental Agriculture. 48, 13871395. 


\section{Pork sensory quality fail rates can be due to pigs from different supply chains varying in breed and rearing environments and fed novel diet ingredients}

$\underline{\text { R J Smits }}{ }^{1}$, A K Lealiifano ${ }^{1}$, H A Channon ${ }^{2}$, J Y C Jolley ${ }^{3}$, D Smyth ${ }^{3}$, A Maronich ${ }^{3}$ and P Smith ${ }^{2}$

${ }^{1}$ Rivalea Australia Pty Ltd, Australia, ${ }^{2}$ Australian Pork Ltd, Australia, ${ }^{3}$ South Australian Research and Development

Institute, Australia

rsmits@rivalea.com.au

Application Sensory assessment using trained panellists has potential to explain why consumers have preferences within a certain pork cut but supplied from different supply chains.

Introduction Fail rates of Australian pork due to sensory quality have been identified through consumer surveys to be attributed to toughness, dryness, bad smell and/or taste and too fatty. Pork which is advertised as being from specific breeds such as Duroc and Berkshire, and raised extensively is often sought by consumers due to marketed characteristics of enhanced taste, juiciness and flavour. Similarly, "novel" diet ingredients, such as macadamia meal, may be used by farmers for added product differentiation. This study compared the sensory performance of pork loins sourced from two different production systems, fed either a conventional wheat/barley grower-finisher diet or the same diet supplemented with macadamia meal. Our hypotheses were consumers from Chinese ethnicity prefer pork from an extensively reared specialist breed meat line, and secondly, that the use of macadamia meal improves consumer preference.

Material and methods The growth study was conducted as a 2 × 2 factorial with 36 female pigs in total sourced from two farms owned by Rivalea Australia Pty Ltd. As per Smits et al. (2019), Farm A used a specialist meat breed, Berkshire $\mathrm{x}$ Duroc and were reared on straw in ecoshelters. Farm B used a commercial breed, Large White $\mathrm{x}$ Landrace $\mathrm{x}$ Duroc reared intensively in a grower-finisher shed. Pigs at each farm were fed either a supplemented finisher diet with macadamia meal (MM) or an un-supplemented (CON) diet. After boning, loins (M. longissimus thoracis et lumborum; $\mathrm{n}=9$ per treatment) were vacuum packed and aged for $7 \mathrm{~d}$ at $1^{\circ} \mathrm{C}$ then frozen. Sensory evaluations were conducted by SARDI, Urrbrae, SA. In Study 1, 65 untrained sensory participants were selected from regular pork consumers with $78 \%$ having a Chinese background. Loin steaks were cut $12-14 \mathrm{~mm}$ thick and cooked on a double plated grill at $160^{\circ} \mathrm{C}$ for 3 mins $10 \mathrm{~s}$ to an internal temperature of $68^{\circ} \mathrm{C}$, rested for $2 \mathrm{mins}$, and trimmed of fat. Each loin steak sample was tested on 10 participants with each tasting 6 samples in a randomised offering. Responses were given a score of 1 to 5 with 1 being least preferred. The remaining meat from each loin was minced and subsampled to form two $20 \mathrm{~g}$ patties per loin for Study 2. In Study 2, loin mince patties were pan fried at $180^{\circ} \mathrm{C}$ until an internal temperature of $65^{\circ} \mathrm{C}$ and assessed by 11 trained sensory participants (random ethnicity). Data was analysed by ANOVA using Senpaq (v6.03, Qi Statistics, 2015).

Results Untrained sensory participants were able to detect significant differences between Farm, but not Diet (Table 1). Participants did not prefer the pork from MM to their usual pork meals $(P<0.01)$. Untrained participants were able to strongly detect farm differences with a preference for steaks from Farm A. There were also Farm $\mathrm{x}$ Diet interactions for flavour, juiciness and overall likelihood to buy $(P<0.05)$ with the response to diet differing between farms. Steaks from the MM supplemented pigs at Farm B were least favoured by untrained participants. In Study 2, trained panellists detected treatment differences for aroma and flavour. Farm A + CON was ranked significantly lower for "Earthy/mushroom" aroma, with Farm B + CON intermediate $(P<0.05)$ to Farm A + MM and Farm B + MM. Pork from Farm A + MM was ranked higher for "Butter" aromas $(P<0.05)$ than CON pork or either diet treatment from Farm B. For flavour, Farm A + CON ranked high for "Oily" attribute. Low preference for pork from Farm B + MM in Study 1 was associated with high scores for "Flaxseed" and "Earthy/mushroom" aromas, "Sour/lactic" flavour and the lowest score for "Juiciness".

Table 1. Mean sensory response (1, least preferred to 5, most preferred) from untrained participants evaluating grilled pork loin steaks taken from Farm A (Berkshire x Duroc, straw based ecoshelter) and Farm B (Large White x Landrace x Duroc, conventional housing), fed conventional (CON) or macadamia supplemented (MM) diets.

\begin{tabular}{llllllcc}
\hline & \multicolumn{2}{c}{ Farm A } & \multicolumn{2}{c}{ Farm B } & \multicolumn{2}{c}{$P$ value } \\
& CON & MM & CON & MM & Farm & Diet & Interaction \\
\hline Aroma & $3.54^{\mathrm{a}}$ & $3.69^{\mathrm{a}}$ & $3.42^{\mathrm{a}}$ & $3.48^{\mathrm{a}}$ & 0.070 & 0.250 & 0.610 \\
Flavour & $3.61^{\mathrm{a}}$ & $3.52^{\mathrm{a}}$ & $3.39^{\mathrm{a}}$ & $3.04^{\mathrm{b}}$ & $<0.001$ & 0.190 & 0.030 \\
Flavour intensity & $3.15^{\mathrm{a}}$ & $3.17^{\mathrm{a}}$ & $3.09^{\mathrm{a}}$ & $2.99^{\mathrm{b}}$ & 0.200 & 0.680 & 0.580 \\
Tenderness & $3.43^{\mathrm{a}}$ & $3.47^{\mathrm{a}}$ & $2.73^{\mathrm{b}}$ & $2.46^{\mathrm{b}}$ & $<0.001$ & 0.340 & 0.180 \\
Juiciness & $3.27^{\mathrm{ab}}$ & $3.59^{\mathrm{a}}$ & $3.11^{\mathrm{b}}$ & $2.45^{\mathrm{c}}$ & $<0.001$ & 0.130 & $<0.001$ \\
Likely to buy & $3.24^{\mathrm{ab}}$ & $3.37^{\mathrm{a}}$ & $3.01^{\mathrm{b}}$ & $2.52^{\mathrm{c}}$ & $<0.001$ & 0.120 & 0.009 \\
\hline
\end{tabular}

${ }_{\mathrm{a}, \mathrm{b}, \mathrm{c}}$ Means within a row with different superscripts differ significantly $(P<0.05)$

Conclusion The untrained consumer sensory study with a high proportion of Chinese participants preferred pork from Farm A, partly supporting the hypothesis. Pork from MM supplemented pigs was least preferred, possibly due to aversions to "flaxseed" and "earthy/mushroom" aromas and "sour/lactic" flavour.

Acknowledgements Supported in part by Australian Pork Limited and Rivalea Australia Pty Ltd.

\section{References}

Smits RJ, Lealiifano AK, Aitken R, Channon HA and Smith P 2019. Advances in Animal Biosciences. 10, s90. 


\section{Colour difference between free range and conventional pork from entire males is greatest during spring}

M Trezona ${ }^{1,2}, K_{\text {L Moore }}^{1,3}$ and I Stensland ${ }^{2}$

${ }^{1}$ Pork Innovation WA Inc., Australia, ${ }^{2}$ Linley Valley Pork, Australia, ${ }^{3}$ KLM Consulting, Australia

mtrezona@craigmostyn.com.au

Application There is an opportunity to improve the consistency of free range pork colour across seasons, however the difference in colour between free range and conventional pork may also provide an opportunity for product differentiation.

Introduction The demand for pork produced in free range (FR) systems is increasing in Australia with major supermarket chains encouraging supply (Pluske, 2019). Currently $30 \%$ of fresh pork produced in Western Australia is from FR systems (Trezona, 2019) and further growth is likely. The way pigs are reared affects meat quality (Martino et al., 2014) and fresh pork colour is an important determinant in consumer purchasing decisions. It was hypothesised that the consistency and characteristics of fresh pork colour from FR systems would differ to that of pork from conventional, intensive systems.

Material and methods From March 2017 to February 2018, 20 entire male carcasses were randomly selected per month $(n=240)$ from a conventional herd (APIQ ${ }^{\circledR}$ accredited, climate controlled, fully slatted birth to bacon production system: Conv) and a FR herd (APIQ $\sqrt{ }{ }^{\circledR}$ Free Range accredited). Data were grouped into season according to calendar months. The herds had the same commercial genetic line (Large White $\mathrm{x}$ Landrace $\mathrm{x}$ Duroc) and were grown under similar feeding programs. Pigs were transported (different slaughter days) to a commercial abattoir (Conv: $2 \mathrm{~h}$ transport; FR: $4 \mathrm{~h}$ transport), off feed for approximately $18 \mathrm{~h}$ and rested for at least $2 \mathrm{~h}$ before slaughter (6-8 h off feed). Twenty-four hours after slaughter the Longissimus thoracis was collected and pH (Cyberscan pH 300, Eutech Instruments Pte Ltd, Singapore) and colour ( $\mathrm{L}^{*}, \mathrm{a}^{*}, \mathrm{~b}^{*}$; Chroma Meter CR-400, Konica Minolta, Japan) were measured at the cut surface, after a 30 minute bloom. Data were analysed by general analysis of variance (GenStat $19^{\text {th }}$ ed, VSN International, Hemel Hempstead, UK). Differences in total pork colour $(\Delta \mathrm{E})$, were calculated from the seasonal averages of $\mathrm{L}^{*}, \mathrm{a}^{*}, \mathrm{~b}^{*}$, where $1<\Delta \mathrm{E}<2$ indicates differences subtle but visible to the trained observer, $\Delta \mathrm{E}>2$ differences are more easily detected by the untrained observer and $\Delta \mathrm{E}>3.5$ clear colour differences are noted (Mokrzycki and Tatol, 2011).

Results Carcass weights tended to be heavier for FR pigs compared to Conv pigs $(69.1 \mathrm{~kg} v s 67.9 \mathrm{~kg}$ for Conv, $P=0.067)$ but backfat depth was similar (FR $9.2 \mathrm{~mm} v s$ Conv $8.9 \mathrm{~mm}, P=0.205)$. $\mathrm{pH}_{24}$ was highest in FR pork $(P=0.003)$ and was higher, regardless of system, during spring $(P<0.001)$. System and season impacted pork colour $(P<0.05)$ and there was an interaction between the two for the aspects of lightness $\left(\mathrm{L}^{*}\right)$ and blue-yellow $(* \mathrm{~b})$ where the greatest difference in colour between FR and Conv pork occurred during spring where FR pork was darker and less yellow than Conv pork. The calculated differences in overall colour $(\Delta \mathrm{E})$ indicated that FR and Conv pork appears similar to the consumer in summer and autumn, differences may be detected during winter while the difference was obvious during spring.

Table 1. Impact of production system, free range (FR) or conventional (Conv) and season on ultimate $\left.\mathrm{pH}^{(\mathrm{pH}} \mathrm{H}_{24}\right)$ and pork colour $\left(\mathrm{L}^{*}, \mathrm{a}^{*}, \mathrm{~b}^{*}\right)$ from entire male finisher pigs.

\begin{tabular}{lcccccccccccc}
\hline & \multicolumn{2}{c}{ Autumn } & \multicolumn{2}{c}{ Winter } & \multicolumn{2}{c}{ Spring } & \multicolumn{2}{c}{ Summer } & & \multicolumn{2}{c}{$P$ value } \\
Syystem & FR & Conv & FR & Conv & FR & Conv & FR & Conv & SED & System & Season & SxS \\
\hline $\mathrm{pH}_{24}$ & $5.50^{\mathrm{b}}$ & $5.45^{\mathrm{a}}$ & $5.53^{\mathrm{bc}}$ & $5.50^{\mathrm{b}}$ & $5.56^{\mathrm{c}}$ & $5.51^{\mathrm{b}}$ & $5.49^{\mathrm{ab}}$ & $5.51^{\mathrm{b}}$ & 0.021 & 0.003 & $<0.001$ & 0.101 \\
$\mathrm{~L}^{*}$ & $51.9^{\mathrm{bcd}}$ & $51.1^{\mathrm{bc}}$ & $50.7^{\mathrm{b}}$ & $52.3^{\mathrm{cd}}$ & $47.5^{\mathrm{a}}$ & $52.7^{\mathrm{d}}$ & $52.5^{\mathrm{cd}}$ & $52.2^{\mathrm{c}}$ & 0.737 & $<0.001$ & $<0.001$ & $<0.001$ \\
$\mathrm{a}^{*}$ & $6.67^{\mathrm{abc}}$ & $6.96^{\mathrm{c}}$ & $6.33^{\mathrm{ab}}$ & $6.82^{\mathrm{bc}}$ & $7.05^{\mathrm{c}}$ & $6.93^{\mathrm{c}}$ & $6.19^{\mathrm{a}}$ & $6.59^{\mathrm{abc}}$ & 0.296 & $<0.001$ & 0.004 & 0.131 \\
$\mathrm{~b}^{*}$ & $5.23^{\mathrm{d}}$ & $4.88^{\mathrm{bcd}}$ & $4.68^{\mathrm{bc}}$ & $5.13^{\mathrm{cd}}$ & $3.22^{\mathrm{a}}$ & $5.22-^{\mathrm{d}}$ & $4.53^{\mathrm{b}}$ & $5.08^{\mathrm{c}}$ & 0.245 & $<0.001$ & $<0.001$ & $<0.001$ \\
$\Delta \mathrm{E}$ & \multicolumn{2}{c}{0.927} & \multicolumn{1}{c}{1.685} & \multicolumn{2}{c}{5.678} & \multicolumn{2}{c}{0.751} & & & & \\
\hline a,b,c,d Means within a row with different superscripts differ significantly $(P<0.05) ;$ SED, standard error of difference of
\end{tabular}
means.

Conclusion Production system affected fresh pork colour. As indicated by $\Delta \mathrm{E}$, differences occurred at subtle levels during autumn and summer, would be evident to the discerning consumer during winter and were obvious during spring. The relationship between $\mathrm{pH}$ and fresh pork colour is well established and the higher $\mathrm{pH}_{24}$ measured for FR pork is consistent with the expectation that pork colour would be darker and differ from the Conv pork. The findings highlight that the variability in FR pork should be managed across season to improve product consistency. Alternatively, the differences found between FR and Conv pork colour may provide an opportunity to differentiate the characteristics of FR pork.

Acknowledgements Supported in part by the Growers Group R\&D grants program administered through the WA Department of Primary Industries and Regional Development's Agribusiness Innovation Fund (GGRD-2015-0069AGSC). The assistance from staff at Linley Valley Pork is gratefully acknowledged.

\section{References}

Mokrzycki WS and Tatol M 2011. Machine Graphics and Vision. 20, 383-411.

Martino G, Mugnai C, Compagnone D, Grotta L, Del Carlo M and Sarti F 2014. Animals. 4, 348-360.

Pluske JM 2019. A final report prepared for Pork Innovation WA Inc. GGRD-2015-0069-AGSC.

Trezona M 2019. A final report prepared for Pork Innovation WA Inc. GGRD-2015-0069-AGSC. 
Review of pig post-mortem inspection and disposition schedules of Australian Standard 4696 A M Pointon $^{1}$, D R Hamilton ${ }^{2}$ and A Kiermeier ${ }^{3}$

${ }^{1}$ APFoodIntegrity Pty Ltd, Australia, ${ }^{2}$ D Hamilton Consulting Pty Ltd, Australia, ${ }^{3}$ Statistical Process Improvement Consulting \& Training Pty Ltd, Australia

andypointon.food@iinet.com.au

Application The review modernises post-mortem inspection procedures and disposition judgement criteria of the Australian domestic standard for hygienic production of pig meat on a consumer risk basis. It enables improved allocation of food safety resources so as to be commensurate with risk to protect public health and underpin market access.

Introduction Modernisation of traditional post-mortem inspection procedures, utilising Codex Alimentarius Commission risk assessment principles, is increasingly occurring internationally (Codex Alimentarius, 2005; Alban et al., 2018). This is in response to redundancy of some traditional inspection procedures due to improvements in animal health status (including zoonoses) and risk from meat-borne hazards that are not detected by post-mortem inspection. It is also increasingly noted that current post-mortem inspection practices (i.e. incision and palpation) may have an adverse effect on product hygiene with contamination of edible tissues by microbial foodborne hazards (Pointon et al., 2018). Australian risk managers now require that alternative procedures should be quantitatively validated for product safety, wholesomeness and adverse effects on monitoring animal health (including zoonoses) and welfare status. The aim of this risk-based review is to assess and revise, where validated, post-mortem inspection procedures and criteria used for carcass disposition judgement for pigs, cattle, sheep and goats in the Australian Standard 4696 (Standards Australia, 2007).

Materials and methods A qualitative risk assessment was initially undertaken to determine and rate hazard:gross abnormality combinations detected by organoleptic post-mortem inspection. Pilot studies were undertaken to examine criteria used for disposition judgement of leading causes of total carcass condemantion. Inspector's hands at two abattoirs were surveyed to quantify bacterial hygiene indicator counts and Salmonella prevalence (Pointon et al., 2018).

Results The qualitative risk assessment identified the need for risk-based studies that were designed to quantify and compare the effectiveness of alternative procedures (Table 1). Methods for each of these are fully detailed by Pointon et al. (2017).

Table 1. Studies developing and validating alternative post-mortem inspection and disposition judgement procedures.

\begin{tabular}{ll}
\hline Risk-based assessments & Approach \\
\hline Effect of visual inspection & Utilise lesion prevalence and sensitivity data to assess equivalence of visual inspection \\
- Food safety "net effect" & Predict contamination added by inspection versus food safety abnormalities removed \\
- Inspector contamination & Determine the rate of Salmonella contamination of inspectors' hands at two abattoirs \\
Kidney enucleation & In-plant comparison of inspection procedures to detect kidney abnormalities \\
Pleurisy & In-plant detection of pleurisy by company Quality Assurance v official inspection \\
Acute pneumonia/pleurisy & Assess lesion stage and carcass microbiological criteria to inform carcass disposition \\
Polyarthritis & Assess lesion stage and carcass microbiological criteria to inform carcass disposition \\
Melanoma & Determine prevalence and distribution of gross abnormalities in affected carcasses \\
\hline
\end{tabular}

Few gross abnormalities of pigs represent a public health risk (Pointon et al., 2018). Minor changes in sensitivity due to visual only inspection results in negligible adverse effect on food safety, wholesomeness and monitoring disease status. Fouteen percent of 56 sets of inspectors' hands at two abattoirs were contaminated with Salmonella (Pointon et al., 2018). The risk of Salmonella contamination after head lymph node incision and palpation compared to visual inspection is estimated to increase by a factor of 214. Kidneys do not need to be enucleated for inspection when not kept for human consumption.

Conclusions A range of alternative post-mortem inspection and disposition procedures have been validated and implemented in Schedules 2 and 3 of the Australian Standard, including routine visual only inspection for all ages of pigs reared in indoor and outdoor production systems (Standards Australia, 2019).

Acknowledgements Supported in part by Australian Pork Limited and the Australian Meat Processors Corporation.

\section{References}

Alban LE, Ruttscheid E, Cordeiro de Sá CVG, Buholzer P, Vieira-Pinto M, Langkabel N, Meemken D, Pointon AM, Hamilton D and Abley M 2018. Fleischwirtschaft International. 2, 8-15.

Codex Alimentarius 2005. Code of Hygiene Practice for Meat (CAC/RCP 58-2005).

Pointon AM, Hamilton DH and Kiermeier A 2017. APL Final Report 2015/023. Australian Pork Ltd, Barton, ACT.

Pointon AM, Hamilton DH and Kiermeier A 2018. Food Control. 90, 222-232.

Standards Australia 2007. Food Regulation Standing Committee Technical Report Series 3. AS 4696:2007. Standards Australia, Sydney, NSW.

Standards Australia 2019. Amended AS 4696:2007. Standards Australia, Sydney, NSW. In Press. 


\section{An improved microbiological monitoring system for the Australian pork industry - an} industry trial

J Y C Jolley $^{1}$, A Kiermeier ${ }^{2}$ and J Sumner ${ }^{3}$

${ }^{1}$ South Australian Research and Development Institute, Australia, ${ }^{2}$ Statistical Process Improvement Consulting Training Pty Ltd, Australia, ${ }^{3} M$ \& S Food Consultants Pty Ltd, Australia

jessica.jolley@sa.gov.au

Application This project provided a unique opportunity to investigate how the Australian meat industry operates process hygiene monitoring. The outcomes for the industry include: reduced costs for compliance and microbiological testing, better utilisation of labour and increased value of collected data.

Introduction Meat testing is an onerous and expensive task for all pork slaughter establishments, and one which currently provides little or no information on process control or food safety. In 2017, a review of the E. coli and Salmonella monitoring (ESAM) program, Product Hygiene Index and Meat Hygiene Assessment programs currently operated by Australian pork export establishments (Jolley et al., 2017) was conducted. As a result of extensive data analysis, alternative microbiological procedures to verify hygienic processing and an industry trial were recommended (Jolley et al., 2019). The aim of the industry trial was to collect data to ascertain the effectiveness of the alternative procedures for process control monitoring.

Material and methods Carcass and bulk meat samples were gathered and tested for indicator organisms as set out in the Microbiological Manual for Sampling and Testing of Export Meat and Meat Products (DAWR, 2018). Primal and offal samples were tested at a carcass equivalent sampling frequency of 1 in 3000 . Primal samples were gathered by sponge swabbing primal cuts prior to packaging and offal samples were tested by excision sampling. The data were statistically analysed using the software program R (version 3.1.3, R Core Team 2015).

Results Three export pork abattoirs gathered a total of 3597 microbiological results (1 762 carcass, 978 bulk meat, 339 primal and 518 offal) from October 2017 to October 2018 (Figure 1).

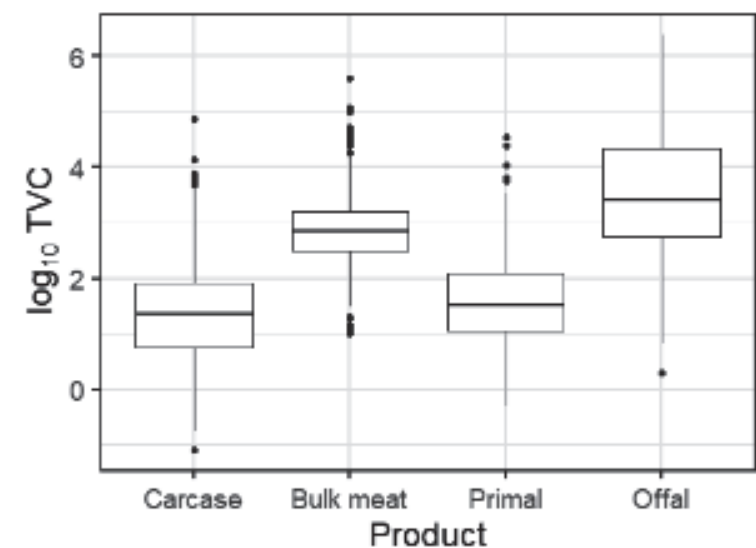

Figure 1. Total Viable Counts (TVCs) for carcasses, bulk meat, primals and offal for all three pork abattoirs combined.
Mean carcass Total Viable Counts (TVCs) ranged between 0.5 to $2 \log \mathrm{cfu} / \mathrm{cm}^{2}$, with primal and bulk meat TVCs approximately one log higher. Offal TVCs approximated $4 \log \mathrm{cfu} / \mathrm{g}$ with higher counts on head offals such as tongues and intestinal offals such as chitterlings. Prevalence of E. coli ranged from 3.5 to $5.0 \%$ on carcasses, less frequently on bulk meat and less frequently again on primals.

A proposed system agreed by industry and the Department of Agriculture and Water Resources (DAWR) involved reducing the frequency of testing indicator organisms, modifying acceptance criteria and the removal of Salmonella testing.

Conclusion It is concluded that: (1) the microbiological profile of porcine carcasses confirms the substantial improvements recorded over recent decades by the ESAM database and by national baseline surveys, (2) the microbiology of bulk meat, primals and offals conforms well with limits imposed by other countries (e.g. New Zealand) and by commerce (e.g. supermarkets) and (3) a proposed system based on testing all product categories will provide better information to establishments and their customers. The pork industry and the DAWR are consulting with overseas markets the possibility of amending the present agreed system based solely on carcass monitoring to include bulk meat, primals and offals.

Acknowledgements Supported in part by the Australian Meat Processors Corporation.

\section{References}

DAWR 2018. Microbiological manual for sampling and testing of export meat and meat products. Version 1.03 Department of Agriculture and Water Resources, Canberra, ACT.

Jolley J, Kiermeier A and Sumner J 2017. AMPC Report 2017-1068. Australian Meat Processor Corporation, North Sydney, NSW.

Jolley J, Kiermeier A and Sumner J 2019. AMPC Report 2018-1070. Australian Meat Processor Corporation, North Sydney, NSW.

R Core Team 2015. R: A language and environment for statistical computing. R Foundation for Statistical Computing, Vienna, Austria. 


\section{Exploratory behaviour and general locomotor activity of Sprague Dawley rats (Rattus norvegicus) following repeated daily exposure to several anticoagulant rodenticides (AR)}

A J Howard $^{1}$, D R Hamilton ${ }^{1}$, I Musgrave ${ }^{2}$ and J Y C Jolley ${ }^{1}$

${ }^{1}$ South Australian Research and Development Institute, Australia, ${ }^{2}$ The University of Adelaide, Australia alexander.howard@sa.gov.au

Application Rodents remain freely active for several days following consumption of AR bait. During this time, baited rodent activity may result in contaminated feed and slaughter stock.

Introduction For decades, anticoagulant rodenticides (AR) have been the dominant rodent control measure used in Australia's intensive livestock enterprises. The internal organs (in particular the liver) and faeces of AR baited rodents contain detectable levels of active ARs, hence baited rodents pose a risk to livestock production through the contamination of slaughter stock. There is insufficient information available on the mobility/foraging behaviours of AR baited rodents, particularly following low dose exposure. This is a critical factor to consider, as freely mobile rats have a greater potential to contaminate feed stocks and the farm environment. This research was undertaken to address this data gap, comparing behavioural effects in Sprague Dawley rats (Rattus norvegicus) following repeated daily exposure to a range of ARs.

Material and methods Thirty female Sprague-Dawley rats (BW $>200$ grams) were weighed and received an AR treatment (either coumatetralyl $(0.22 \mathrm{mg} / \mathrm{kg})$, brodifacoum $(0.11 \mathrm{mg} / \mathrm{kg})$, bromadiolone $(0.20 \mathrm{mg} / \mathrm{kg})$, flocoumafen $(0.41$ $\mathrm{mg} / \mathrm{kg}$ ) or a drinking water control) via oral gavage. AR dose rates were verified through liquid chromatography and tandem mass spectrometry (LC-MS-MS). Animal ethics approval was achieved through the use of sub-LD $\mathrm{D}_{50}$ dose rates for each AR treatment, following an initial pilot study with limited animals to verify that these dose rates were not overtly lethal. Open Field (OF) testing was used to assess the exploratory behaviour of AR dosed rats at d 0, 4, 7 and 13 following treatment. Individual rats were placed in an enclosed circular field (one-metre in diameter) and activity was recorded using a camera suspended above the arena for a minimum of five minutes. Open source software programs (ImageJ and MouseMove) were used to process the footage and measure the total distance travelled by a rat (Samson et al., 2015).

Results AR compounds were found to be highly unstable in solution, resulting in variable dose rates being administered. Coumatetralyl degraded severely in solution, with the cumulative dose rate found to equal roughly $2 \%$ of $\mathrm{LD}_{50}$. Degradation of the remaining AR's was less severe, with actual cumulative doses ranging from $17-88 \%$ of the intended oral $\mathrm{LD}_{50}$ of each compound. In the pilot study, rats fed up to $88 \%$ of $\mathrm{LD}_{50}$ of bromadiolone $(0.99 \mathrm{mg} / \mathrm{kg})$ retained normal activity levels and feeding behaviours up to d 7. After d 7, mobility, feeding behaviour and general health status declined rapidly and they were euthanised at $\mathrm{d} 11 \& 13$ respectively. As a result, the bromadiolone dose rate was reduced for the main feeding trial to $0.20 \mathrm{mg} / \mathrm{kg}$. No significant differences $(P>0.05)$ were observed in the OF total travel distance of rats exposed to any of the other AR treatments $\left(17-60 \%\right.$ of $\left.\mathrm{LD}_{50}\right)$. Further, rats from all treatment groups, except for high dose bromadiolone, gained weight steadily throughout the trial, indicating that feeding behaviours remain stable following sub-lethal AR exposure.

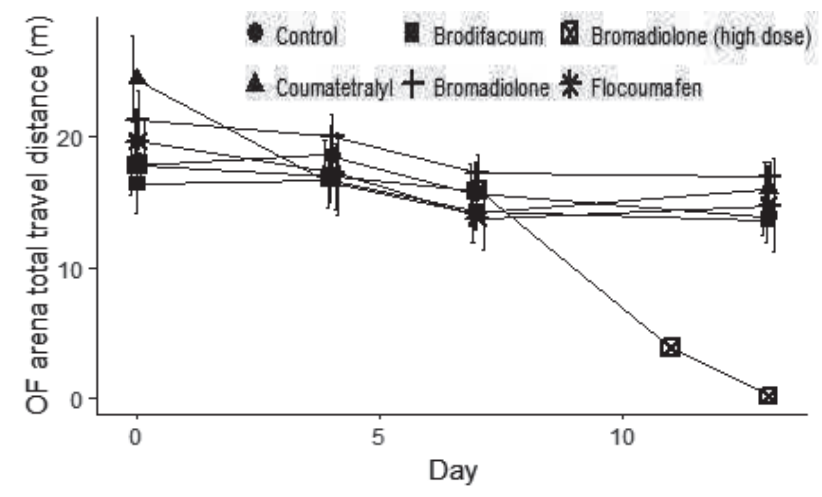

Figure 1. Mean total distance (metres) travelled by AR dosed rats during OF testing.

Conclusion This research demonstrates that rats exposed to repeated low doses of ARs retain their normal exploratory and feeding behaviours. Following repeated administration of a higher dose of bromadiolone, a considerable delay ( 7 days) was observed before the onset of incapacitating symptoms. This delay results in a potential window where rodents that have consumed doses of ARs remain active, and therefore pose a risk to livestock production through the persistence of AR residues in the farm environment and potentially in slaughter stock.

\section{References}

Samson AL, Ju L, Ah Kim H, Zhang SR, Lee JA, Sturgeon SA, Sobey GG, Jackson SP and Schoenwaelder 2015. Scientific Reports. 5, 16171. 


\section{Provision of straw or cotton rope to sows may result in more piglets being born alive}

R S Morrison $^{1}$, L E Edwards ${ }^{2}, \mathrm{~S}$ L Beer ${ }^{1}, \mathrm{~K}$ J Plush ${ }^{3}$ and R E Doyle ${ }^{2}$

${ }^{1}$ Rivalea Australia Pty Ltd, Australia, ${ }^{2}$ Animal Welfare Science Centre, The University of Melbourne, Australia, ${ }^{3}$ SunPork Group, Australia

rmorrison@rivalea.com.au

Application Provision of straw or cotton rope to sows housed in farrowing crates prior to farrowing may result in more piglets being born alive.

Introduction Enhancing the welfare of sows in farrowing systems is a priority for Australian pork producers. Previous research conducted by the authors showed that peri-natal piglet survival was increased when sows were provided with approximately $1 \mathrm{~kg}$ lucerne hay/d prior to farrowing (Doyle et al., 2017). The aim of this experiment was to investigate non-nutritive and nutritive nest building materials (i.e. materials that can be orally manipulated, rooted and pawed by the sow). It was hypothesised that the provision of nest building material would result in more piglets being born alive.

Material and methods A total of 724 mixed parity sows (gilts - parity 7) (Large White x Landrace Primegro ${ }^{\mathrm{TM}}$ Genetics, Corowa, NSW) over seven time replicates were allocated to treatment with parity balanced between treatments. The sows were housed in standard farrowing crates and treatments were Control - no enrichment; Cotton rope $(30 \mathrm{~cm}$ long x $3 \mathrm{~cm}$ thick cotton rope tied to the top rail of the farrowing crate; hung vertically next to the feed trough in front of the sow); 1 $\mathrm{kg}$ long-cut straw placed in feed trough daily; $\sim 1 \mathrm{~kg}$ lucerne hay placed in feed trough daily. The sows were provided the nest building material approximately two days prior to estimated farrowing date (d 115 gestation). Straw and lucerne were replenished daily prior to farrowing if required. The use of enrichment during the nesting period (18 h prior to farrowing) and farrowing duration were recorded on video for 18 sows/treatment. The total number of piglets born alive, stillborn and mummified were recorded for all sows. Stillborn piglets were judged on the basis that they were fully formed at farrowing, covered in foetal membrane and had fully formed eponychia on their hooves. Data were analysed Univariate General Linear Model, using each sow/litter as the experimental unit with parity, treatment and their interaction as fixed effects and time replicate as a random term (IBM SPSS Statistics for Windows, v25.0. Armonk, NY).

Results There was evidence of use of nesting material; $62 \%$ of sows used the straw, 36\% of sows used the lucerne and $33 \%$ used the cotton rope during the nesting period. There was a tendency $(P=0.053)$ for treatment effects on the number of stillborn piglets. The provision of nest building material did not significantly $(P>0.05)$ impact on farrowing behaviour (e.g. farrowing duration).

Table 1. The effects of the provision of nest building materials on sow mean reproductive performance

\begin{tabular}{lcccccc}
\hline & Control & Cotton rope & Straw & Lucerne & SEM & $P$ value \\
\hline Total born & 13.3 & 12.9 & 13.2 & 13.7 & 0.12 & 0.485 \\
Piglets born alive $^{1}$ & 11.8 & 12.1 & 12.1 & 11.8 & 0.11 & 0.058 \\
Stillborn piglets $^{1}$ & 1.0 & 0.8 & 0.7 & 0.9 & 0.04 & 0.053 \\
Mummies $^{1}$ & 0.4 & 0.2 & 0.3 & 0.3 & 0.02 & 0.572 \\
\hline
\end{tabular}

${ }^{1}$ Number of piglets total born used as covariate; SEM, standard error of the mean.

Conclusion The hypothesis was supported. These results suggest that nesting materials may affect stillbirth rate, particularly straw and cotton rope, but clearly further research is required to understand the mechanism, particularly since there was no treatment effect on duration of parturition. The provision of nest building material may render protection to piglets through alternative pathway(s).

Acknowledgements Supported in part by Pork CRC Limited, Research and Innovation Farms staff at Rivalea Australia Pty Ltd and the Animal Welfare Science Centre, The University of Melbourne.

\section{References}

Doyle RE, Ralph CR, Edwards LE, Morrison RS, Cronin GM and Plush KJ 2017. Animal Production Science. 57, 2439. 


\title{
Positive human contact reduces avoidance behaviour of gestating sows during routine husbandry procedures
}

\author{
M Hayes $^{1}$, L M Hemsworth ${ }^{1}$, R S Morrison ${ }^{2}$, M Rice ${ }^{1}$ and P H Hemsworth ${ }^{1}$
}

${ }^{1}$ Animal Welfare Science Centre, The University of Melbourne, Australia, ${ }^{2}$ Rivalea Australia Pty Ltd, Australia

mehayes@student.unimelb.edu.au

\begin{abstract}
Application Providing pigs with regular opportunities for positive interactions with humans may help to reduce animal stress and improve ease of handling by stockpeople during routine husbandry practices imposed in commercial systems.
\end{abstract}

Introduction Human-animal interactions are a critical feature of routine management in pork production systems. Frequent negative interactions result in high levels of animal fear towards humans, which has implications for animal welfare and productivity (Hemsworth and Coleman, 2011). In addition, the stockperson's job is likely more difficult when working with fearful animals exhibiting high avoidance behaviour towards humans. While the impacts of negative humananimal interactions are well known, the effects of positive interactions have been less studied, however there is evidence that positive interactions influence how animals respond to stressful events involving humans. For example, previous positive human contact has been shown to improve ease of handling and reduce heart rate in calves during loading for transport (Lensink et al., 2001), reduce heart rate and salivary cortisol concentrations in lambs following tail-docking (Tosi and Hemsworth, 2002), and reduce heart rate and restless behaviour in cows during rectal palpations (Waiblinger et al., 2004). These studies provide evidence that positive human interactions can influence how other farm animals respond to stressful events involving humans, and so the aim of this study was to determine if positive human interaction could be used to reduce pigs' fear of humans during routine husbandry practices. It was hypothesised that brief, daily positive human contact would reduce pigs' avoidance of the technician during pregnancy testing and vaccination.

Material and methods A factorial design with two main effects was used to study 360 group-housed sows (Large White x Landrace Primegro ${ }^{\text {TM }}$ Genetics, Corowa, NSW) over two-time replicates: 1 . Human contact - Positive human contact $(+\mathrm{HC})$ and Control (C); and 2. Parity - Parity 1-2 and Parity 3+. The + HC treatment was imposed for 2 min daily throughout gestation and involved one of five stockpeople walking slowly through the group pens and patting sows. Sows were housed in groups of 15 in partially slatted, non-bedded and non-enriched pens. Using CCTV footage, the withdrawal responses of sows towards the technician before and after pregnancy testing (wk 6 gestation) and vaccination (wk 12 gestation) were observed. A sow was classed as withdrawing if she moved away from the technician within 3 sec of his approach and within $3 \mathrm{sec}$ after being vaccinated/pregnancy tested. The number of tactile interactions (nosing and chewing the technician) throughout the imposition of pregnancy testing and vaccination in the group pens was also measured. The technician was one of the stockpeople responsible for delivering the $+\mathrm{HC}$ treatment each day. The experimental unit was a pen of sows $(n=24)$, and behavioural variables were analysed by ANOVA with treatment, parity and replicate as fixed effects (IBM SPSS Statistics for Windows, v24.0. Armonk, NY).

Results Data are presented as estimated marginal means \pm SEM. Fewer + HC sows withdrew when the technician approached to conduct pregnancy testing compared to $\mathrm{C}$ sows (proportion of sows from the pen withdrawing, $0.12 \mathrm{vs}$ $0.37 \pm 0.04 ; P=0.001)$ and after the test had been conducted $(0.16$ vs $0.52 \pm 0.03 ; P<0.001)$. Fewer $+\mathrm{HC}$ sows also withdrew when the technician approached to conduct vaccination $(0.43 v s 0.67 \pm 0.05 ; P=0.003)$. There was no difference in withdrawal responses between $+\mathrm{HC}$ and $\mathrm{C}$ sows after vaccination, however $90 \%$ of all sows withdrew after receiving their injection indicating a high level of fear towards this practice. More younger sows withdrew before $(0.32$ vs 0.18 $\pm 0.04 ; P=0.043)$ and after $(0.39$ vs $0.29 \pm 0.03 ; P=0.031)$ pregnancy testing in comparison to older sows, but there was no effect of parity on withdrawal responses around vaccination. No sows interacted with the technician during vaccination, however during pregnancy testing there were more tactile interactions initiated by $+\mathrm{HC}$ sows than $\mathrm{C}$ sows (number of interactions, 17 vs $6 \pm 1.9 ; P \leq 0.001$ ). Parity did not influence sow-initiated interactions with the technician. There were no significant parity $\mathrm{x}$ human contact interactions on any variables measured.

Conclusion This study provides evidence that $+\mathrm{HC}$ reduced fear (based on avoidance behaviour) of sows towards pregnancy testing and vaccination. Reduced fear is obviously a positive outcome in terms of animal welfare, but may also improve ease of handling and management of pigs by stockpeople. Clearly further research is required to examine the effects of regular $+\mathrm{HC}$ on the stress responses of pigs to husbandry practices imposed in commercial systems, including physiological responses, and the impact of $+\mathrm{HC}$ on more invasive practices such as those requiring surgical intervention.

Acknowledgements Supported in part by Pork CRC Limited and the assistance of Australian Pork Limited and Rivalea Australia Pty Ltd for facilitating this project is acknowledged.

\section{References}

Hemsworth PH and Coleman GJ 2011. Human-livestock interactions: 2nd Edition. CABI, Wallingford, UK.

Lensink BJ, Fernandez X, Cozzi G, Florand L and Veissier I 2001. Journal of Animal Science. 79, 642-652.

Tosi MV and Hemsworth PH 2002. In Proceedings of 36th International Congress of the ISAE (Ed: Koene P) The

Organising Committee, Wageningen, The Netherlands. p. 129

Waiblinger S, Menke C, Korff J and Bucher A 2004. Applied Animal Behaviour Science. 85, 31-42. 


\section{Corticosteroid administration can mitigate the stress response of post-weaned pigs exposed to mixing}

$\underline{\text { S O Sterndale }}^{1}$, D W Miller ${ }^{1}$, J P Mansfield ${ }^{1}$, J C Kim ${ }^{1,2}$ and J R Pluske ${ }^{1}$

${ }^{1}$ Murdoch University, Australia, ${ }^{2}$ AB Vista, UK

samantha@sterndale.com

Application Dexamethasone administration has the ability to mitigate stress response by manipulating neurotransmitters and hormones that attenuate acute stress responses in weaner pigs

Introduction Weaning piglets involves separation from the sow and mixing of unfamiliar pigs in a new environment. This can lead to vigorous fighting, social stress and often a reduction in weight gain (Puppe et al., 1997). Dexamethasone (DEX) is a corticosteroid that has been shown to attenuate stress responses by reducing hypothalamic-pituitary-adrenal (HPA) activity via negative feedback, as well as improving growth (Wooten et al., 2019). The hypothesis tested in this study was that DEX administration would ameliorate stress responses, as indicated by reduced cortisol and urea nitrogen secretion, increased brain derived neurotrophic factor (BDNF) and weight gain, when weaner pigs were mixed with nonlittermate counterparts. BDNF decreases during an acute stress response which can have cascading effects on the neuroendocrine system as it has protective effects on neurons and stimulates the release of certain neurotransmitters such as serotonin and dopamine (Martinowich and Lu, 2008). Plasma urea nitrogen (PUN) is used to measure the amount of waste product produced by the liver as the body breakdown proteins.

Material and methods At weaning (d 0) 96 male piglets $(6.5 \pm 0.9 \mathrm{~kg})$ were allocated into pens with their litter mates (4/pen) and allowed to acclimatise for $14 \mathrm{~d}$. At d 14 of the study, pigs were allocated to a 2 x 2 factorial design with the factors being (1) without/with mixing of non-littermates, and (2) without/with DEX administration. The study finished on d 28. On d 12 and 13, pigs in the DEX treatments were given intramuscular (i.m.) injections of $0.2 \mathrm{mg} / \mathrm{kg} \mathrm{DEX} \mathrm{(5}$ $\mathrm{mg} / \mathrm{mL}$, Dexapent, Troy Illium, NSW, Australia) at 0600 and $1800 \mathrm{~h}$ (total 4 doses/pig) and the pigs in the control treatment were given the same volume of saline i.m. On d 14 at 0800 h, 48 pigs were mixed with non-litter mates and 48 pigs remained with their littermates. Individual body weights were measured weekly, and blood was collected at $1200 \mathrm{~h}$ on d 11, 14 and 16. Commercially available ELISA kits were used to analyse plasma cortisol (Enzo Life Sciences, Cat No. ADI-900-071, NY, USA) and BDNF (MyBioSource, Cat No. MBS163950, CA, USA). PUN was analysed using Beckman Coulter/Olympus Reagent kit (OSR6134). Data were analysed by two-way analysis of variance using SPSS (IBM SPSS Statistics for Windows, v21.0. Armonk, NY) with main effect means being presented (Table 1).

Results In the week pigs were given DEX or saline (d 7-14), average daily gain was higher in the DEX-treated pigs (361 vs $296 \mathrm{~g} / \mathrm{d}$ respectively, $P=0.002)$. However, this improvement was not seen thereafter $(\mathrm{d} 14-28)$ in the study. Plasma cortisol was higher $(P=0.001)$ in pigs exposed to mixing on the day of mixing (d 14) and $2 \mathrm{~d}$ after mixing (d 16). The pigs given DEX tended $(P=0.100)$ to have lower cortisol at d 14 regardless of the mixing treatment. These results suggest that mixing unfamiliar pigs initiates a stress response and DEX treatment has the ability to reduce the severity of the response via negative feedback on the HPA axis. Furthermore, BDNF levels were higher $(P=0.043)$ in DEX-treated pigs at d 16 consistent with its ameliorative effects on neurons and stimulation of release of certain neurotransmitters. Levels of PUN were lower in DEX-treated pigs compared to control pigs at d $14(3.1 v s 4.2 \mu \mathrm{mol} / \mathrm{L}, P=0.060)$ and d $16(3.2 v s$ $4.1 \mu \mathrm{mol} / \mathrm{L}, P=0.001)$. These results suggest that DEX has the ability to either improve efficiency of nitrogen utilisation or reduce muscle protein break down associated with the stress (Wooten et al., 2019).

Table1. Plasma results in weaner pigs given saline or dexamethasone $(0.2 \mathrm{mg} / \mathrm{kg}(5 \mathrm{mg} / \mathrm{mL})) \mathrm{with} /$ without acute mixing stress

\begin{tabular}{lcccccccc}
\hline & \multicolumn{2}{c}{ Treatment } & \multicolumn{2}{c}{ Stressor } & \multicolumn{3}{c}{$P$ value } \\
& Control & DEX & Mixing & Non-mixing & SEM & Treatment & Stressor & T x S \\
\hline Cortisol d 14 $(\mathrm{ng} / \mathrm{ml})$ & 16.0 & 10.6 & 20.2 & 7.5 & 2.67 & 0.100 & 0.001 & 0.754 \\
Cortisol d 16 $(\mathrm{ng} / \mathrm{ml})$ & 21.0 & 17.2 & 26.2 & 12.0 & 2.30 & 0.240 & 0.001 & 0.659 \\
BDNF d 16 $(\mathrm{ng} / \mathrm{ml})$ & 3.08 & 5.58 & 4.38 & 4.27 & 0.85 & 0.043 & 0.927 & 0.697 \\
\hline
\end{tabular}

Conclusion Administration of DEX in weaner pigs exposed to a mixing stressor, increased BDNF, improved nitrogen utilisation and decreased cortisol, presumably via negative feedback on the HPA axis. However, there was no improvement in performance after DEX administration possibly due to the repeated DEX injections resulting in immunosuppressive effects and/or catabolism of the adipose tissue. Nevertheless, DEX administration in weaner pigs warrants further investigation in an altered administration protocol.

Acknowledgments Supported in part by Australian Pork Limited.

\section{References}

Martinowich K and Lu B 2008. Neuropsychopharmacology Reviews. 33, 73-83

Puppe B, Tuchscherer M and Tuchscherer A 1997. Livestock Production Science. 48, 157-164.

Wooten H, McGlone J, Wachtel M, Thompson G, Rakhshandeh AR and Rakhshandeh A 2019. Animal. 10, 1-10. 


\section{Minimal increases in space allowance during the nursery phase in a commercial setting improves performance and health}

D L Turpin $^{1,2}$ and J R Pluske ${ }^{1}$

${ }^{1}$ Murdoch University, Australia, ${ }^{2}$ Pork Innovation Western Australia, Australia

Diana.Turpin@murdoch.edu.au

Application Pig flow strategies which increase space allowance should not be underestimated in their ability to improve performance and health, especially as pork industries worldwide move towards reducing antimicrobial usage.

Introduction Optimising the pig's environment through appropriate stocking density as a strategy to eliminate unnecessary stress and minimise disease has come to light again. In this regard, while space studies for grow-finish pigs are very important, the nursery phase is often overlooked. The Model Code of Practice for the Welfare of Animals: Pigs, Third Edition (PISC, 2008) stipulates that a space allowance of $0.3 \mathrm{~m}^{2}$ per pig is required for weaner housing assuming an average weight of $30 \mathrm{~kg}$ at the end of the nursery period (10 weeks of age). In a previous Canadian study looking at space allowances below $0.3 \mathrm{~m}^{2}$, even a small difference of $0.04 \mathrm{~m}^{2}$ (i.e. $0.26 \mathrm{~m}^{2}$ versus $0.3 \mathrm{~m}^{2}$ ) positively influenced growth during the first five weeks after weaning (Roy, 2017). The current study tested the hypothesis that increases in space allowance in the nursery phase above $0.3 \mathrm{~m}^{2}$ per pig would improve post-weaning performance and health as measured by mortalities and the number of removals due to death or hospital treatment. This study was conducted in a commercial setting using realistically achievable stocking densities.

Material and methods A total of 1260 newly-weaned pigs were allocated to one of three space allowance groups on the day of weaning $(\mathrm{d} 0)$, which was achieved by varying the numbers of pigs per pen. The three space allowances were: i) $0.3 \mathrm{~m}^{2}, 40$ pigs per pen; ii) $0.35 \mathrm{~m}^{2}, 35$ pigs per pen; and iii) $0.4 \mathrm{~m}^{2}, 30$ pigs per pen $(\mathrm{n}=12)$. This was a commercial study and group size could not be controlled between treatment groups. A commercial weaner diet was offered ad libitum. Average daily gain (ADG) and average daily feed intake (ADFI) were compared on different days throughout the nursery phase between treatments using the GLM procedures of SPSS (IBM SPSS Statistics for Windows, v24.0. Armonk, NY) with pen as the experimental unit. Removals due to death or hospital treatment were recorded and compared using Chisquared analysis.

Results During the experiment, the shed experienced an unexpected disease challenge. If pigs were removed from experimental pens, it was recorded, and another pig of a similar weight was used to maintain the stocking density. This may have impacted on social hierarchy and health status. Removals in the $0.3 \mathrm{~m}^{2}$ pens were nearly double that of the 0.35 $\mathrm{m}^{2}$ and $0.4 \mathrm{~m}^{2}$ pens $(7.8 \%$ vs $4.3 \%$ and $4.0 \%$ respectively, $P=0.05)$. Furthermore, pigs exposed to the lowest stocking density finished heavier than pigs exposed to the highest stocking density (Table $1, P<0.05$ ). Pigs from the $0.35 \mathrm{~m}^{2}$ pens tended to finish heavier than the $0.3 \mathrm{~m}^{2}$ pigs $(P<0.1)$. Pigs stocked at $0.35 \mathrm{~m}^{2}$ and above grew faster than pigs stocked at $0.3 \mathrm{~m}^{2}$ between $\mathrm{d} 14$ and 40 of the experiment. There were no treatment differences for growth in the first two weeks of the experiment, and there were no treatment differences for ADFI and FCR.

Table 1. Average body weight (BW), average daily gain (ADG), average daily feed intake (ADFI) and feed conversion ratio (FCR) per pen for three different space allowances in the nursery phase.

\begin{tabular}{|c|c|c|c|c|c|}
\hline \multicolumn{6}{|c|}{ Space allowance $\left(\mathrm{m}^{2}\right)$} \\
\hline & 0.30 & 0.35 & 0.40 & SEM & $P$ value \\
\hline \multicolumn{6}{|l|}{ BW (kg) } \\
\hline d 0 & 6.5 & 6.6 & 6.5 & 0.13 & 0.947 \\
\hline d 40 & $24.5^{\mathrm{ax}}$ & $25.3^{\text {aby }}$ & $25.9^{\mathrm{b}}$ & 0.33 & 0.024 \\
\hline \multicolumn{6}{|l|}{$\operatorname{ADG}(g / d)$} \\
\hline d $0-7$ & 119 & 143 & 117 & 26.0 & 0.729 \\
\hline d 8-14 & 384 & 367 & 413 & 20.6 & 0.297 \\
\hline d $15-40$ & $558^{\mathrm{a}}$ & $585^{\mathrm{b}}$ & $603^{b}$ & 8.6 & 0.003 \\
\hline ADFI d 0-40 (g/d) & 611 & 621 & 643 & 15.3 & 0.330 \\
\hline FCR d 0-40 (g/g) & 1.36 & 1.33 & 1.33 & 0.03 & 0.675 \\
\hline
\end{tabular}

a,b Means within a row with different superscripts differ significantly $(P<0.05)$; ${ }^{\mathrm{x}, \mathrm{y}}$ Means within a row with different superscripts are a trend $(P<0.1)$; SEM, standard error of the mean.

Conclusion Outcomes of this study demonstrate that by increasing space allowance by $16.6 \%$ in the nursery phase, improvements in growth and health can be achieved.

Acknowledgements Supported in part by the Agricultural Produce Commission, Pork Producers' Committee.

\section{References}

PISC 2008. Model Code of Practice for the Welfare of Animals: Pigs, Third Edition. CSIRO Publishing, Collingwood, VIC, Australia.

Roy RC, Kaur R, Seddon YM, Beaulieu AD, Edwards SA, Boussieres D and Brown JA 2017. National Hog Farmer. May 30. 
The analgesic effect of three pain relief protocols on behaviour in castrated piglets

N Matsumoto $^{1}$, D L Turpin ${ }^{1,2}$, A Ballares ${ }^{3}$, R H Dunlop ${ }^{1}$, G Chuck $^{1}$, K Savage ${ }^{1}$ and K Nairn ${ }^{1,3}$

${ }^{1}$ Apiam Animal Health, Australia; ${ }^{2}$ Murdoch University, Australia; ${ }^{3}$ Peel Pork, Australia

nina.matsumoto93@gmail.com

Application Effective and practical pain relief options for use during piglet castration in Australian commercial production systems need further exploration as the treatments did not reduce "castration pain associated" behaviours.

Introduction In Australia, some piggeries castrate male piglets to manage adverse behaviour and improve eating quality. Castration is a painful surgical procedure routinely performed at 7-10 d of age without analgesia. Due to welfare concerns, the use of pain relief during castration is broadly studied with previous focus on intramuscular non-steroidal antiinflammatory drugs (NSAID), local anaesthetics, topical anaesthetics and other multi-modal combinations. In Australian sheep and cattle, Buccalgesic ${ }^{\circledR}(10 \mathrm{mg} / \mathrm{mL}$ Meloxicam, Troy Laboratories Pty Ltd, Glendenning, NSW), a NSAID administered into the cheek pouch, and topical anaesthetic agents such as Tri-Solfen ${ }^{\circledR}(40.6 \mathrm{mg} / \mathrm{mL}$ Lignocaine, 4.2 $\mathrm{mg} / \mathrm{mL}$ Bupivacaine, Bayer Australia Ltd, Pymble, NSW) can be used to provide effective analgesia during castration (Lomax and Windsor, 2013; Van der Saag et al., 2018). However, the efficacy of these products for castration in piglets has only partially been assessed (Lomax et al., 2017). In this study it was hypothesised that the aforementioned analgesics would reduce behaviours associated with pain after castration in piglets and a multi-modal approach would act additively.

Materials and methods Seventy-one male piglets from a commercial farm in Western Australia within 6 litters were allocated to treatment groups: control $(\mathrm{CON})$, no analgesia during castration $(\mathrm{n}=16)$; topical anaesthetic (TA) where $1 \mathrm{ml}$ of Tri-Solfen ${ }^{\circledR}$ was sprayed onto the spermatic cords and inside the wound sites ( $\left.\mathrm{n}=16\right)$; buccal meloxicam (BM) where piglets received $0.4 \mathrm{ml} / \mathrm{kg}$ of Buccalgesic ${ }^{\circledR} 30$ minutes prior to castration $(\mathrm{n}=18)$, or Buccalgesic ${ }^{\circledR}$ and Tri-Solfen ${ }^{\circledR}$ (BMTA) (n=14). Eye temperatures (not presented) were measured 30 minutes prior, immediately after and 240 minutes after the castration procedure which ensured similar handling of all treatment groups. Behavioural monitoring was performed by a blinded observer using focal-scan sampling at specific time points before and after the castration. At each time point the piglets were observed for 5 minutes and their behaviours recorded every 30 seconds. For the data analysis the behaviours were grouped into "castration pain associated" (e.g. scratching, spasms) and "non-castration pain associated" (eg. sleeping, playing) (Hay et al., 2003). Expression of behaviours were compared using the mixed linear model function (IBM SPSS Statistics for Windows, v24.0. Armonk, NY) with piglet as the experimental unit.

Results There were no treatment differences for "castration pain associated" behaviours (Figure 1, $P>0.05$ ), however across all treatment groups, "castration pain associated" behaviours were present at levels higher than they were prior to castration up to $72 \mathrm{~h}$ post-surgery $(P<0.05)$. Significantly more BMTA piglets were observed sucking immediately after castration $(P<0.05)$ and CON piglets spent significantly more time sleeping at the same time point $(P<0.05)$. There were no treatment differences for the other individual behaviours $(P>0.05)$.

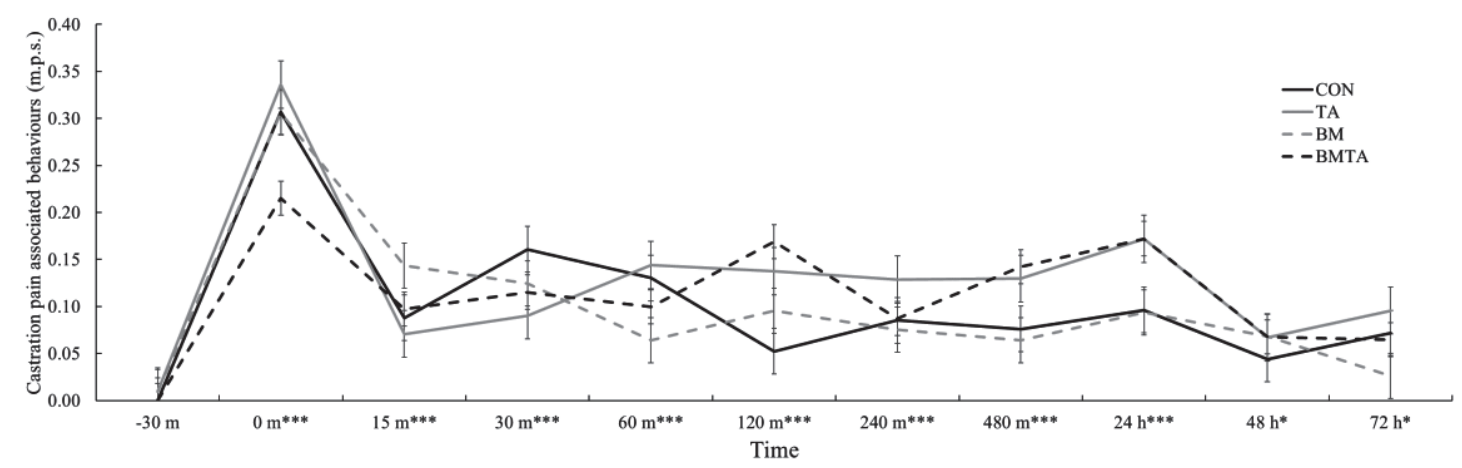

Figure 1. Castration pain associated behaviours over time for controls and three different pain relief protocols

Conclusion Findings from this research demonstrate that castration is indeed a painful procedure with piglets displaying pain associated behaviours for up to $72 \mathrm{~h}$ after castration. However, neither buccal meloxicam nor topical anaesthesia provided a significant improvement to pain after castration as indicated by the selected behavioural measures in this study. A lack of improvement could be associated with the dose, administration method or administration timing of the products and further exploration into alternative protocols is warranted.

Acknowledgments Supported in part by the Apiam Animal Health Production Animal Intern program.

\section{References}

Lomax S, Harris C, Windsor PA and White PJ 2017. PLoS One 12, 1-8.

Lomax S and Windsor PA 2013. Journal of Animal Science. 91, 4945-4952.

Van der Saag D, Lomax S, Windsor PA, Taylor C, Thomson P, Hall E and White PJ 2018. Animal. 12, 2373-2381.

Hay M, Vulin A, Génin S, Sales P and Prunier A 2003 Applied Animal Behaviour Science. 82, 201-218. 


\section{Validation of routine visual only post-mortem inspection of pigs in Australia}

A M Pointon $^{1}$, D R Hamilton ${ }^{2}$ and A Kiermeier ${ }^{3}$

${ }^{1}$ APFoodIntegrity Pty Ltd, Australia, ${ }^{2}$ D Hamilton Consulting Pty Ltd, Australia, ${ }^{3}$ Statistical Process Improvement Consulting \& Training Pty Ltd, Australia

andypointon.food@iinet.com.au

Application Routine visual only post-mortem inspection of all commercially-reared pigs provides opportunity to improve food safety, while ensuring wholesomeness of edible meat and enabling more effective monitoring of animal health (including zoonoses) and welfare status.

Introduction Review of traditional post-mortem inspection procedures, utilising Codex Alimentarius Commission risk assessment guidelines, has been conducted for pigs in Australia (Pointon et al., 2018). This study aimed to quantify any adverse effect arising from routine visual only inspection on food safety, wholesomeness and animal health and welfare status when compared with procedures of the current Australian Standard AS4696 (Standards Australia, 2007).

Materials and methods Gross abnormality prevalence and sensitivity of detection data for different post-mortem inspection (PMI) procedures were used to calculate differences in gross abnormality non-detection rates to compare the effectiveness of procedures. Methods of calculation for each of these comparisons are provided in Table 1; extracted from Pointon et al. (2017).

Results Negligible effects on non-detection rates were estimated to arise from routine visual only inpsection (Table 1).

Table 1. Estimated effect of alternative inspection procedures on non-detection rates of gross abnormalities

\begin{tabular}{lcccc}
\hline $\begin{array}{l}\text { Gross abnormality and disease } \\
\text { (including zoonoses) }\end{array}$ & $\begin{array}{c}\text { Prevalence } \\
\text { in Australia }\end{array}$ & \multicolumn{2}{c}{$\begin{array}{c}\text { PMI Sensitivity of detection } \\
\text { Palpate }\end{array}$} & $\begin{array}{c}\text { Difference in Non-Detection } \\
\text { Rate/10,000 }\end{array}$ \\
\hline $\begin{array}{l}\text { Public health risk-meat-borne hazard associated } \\
\text { Carcass abscess, pyaemia }\end{array}$ & $0.6 \%$ & $0.82^{3}$ & $0.86^{3}$ & $-2.1^{2}$ and $7.8^{4}$ \\
Cultiple pulmonary abscesses & $<0.01 \%$ & $0.82^{3}$ & $0.86^{3}$ & $0^{2}$ \\
Peritonitis - acute \& localised & $1.3 \%$ & $0.8^{5}$ & $0.8^{5}$ & $0^{2}$ \\
Liver abscesses & $<0.1 \%$ & $0.94^{4}$ & $0.87^{4}$ & $3^{2}$ \\
Gross abnormalities affecting wholesomeness and animal health and welfare status & \\
Arthritis & $0.7 \%$ & $0.72^{4}$ & $0.61^{4}$ & $7.7^{2}$ \\
Ascaris suum & $3.3 \%$ & $0.97^{3}$ & $0.98^{3}$ & $0^{2}$ \\
Pericarditis & $2.6 \%$ & $0.8^{5}$ & $0.8^{5}$ & $0^{2}$ \\
Pleurisy & $9 \%$ & Visual unchanged & $0^{4}$ \\
\hline
\end{tabular}

${ }^{1}$ Pointon et al., 2008; ${ }^{2}$ Pointon et al., 2017; ${ }^{3}$ EFSA, 2011; ${ }^{4}$ Hamilton et al., 2002; ${ }^{5}$ Willeberg et al., 1997.

Conclusions Routine visual only inspection has a negligible adverse effect on non-detection rates for gross abnormalities affecting food safety, wholesomeness and animal health and welfare status. Routine visual inspection for all age-groups of intensively and extensively reared domestic pigs has been judged as equivalent with Schedule 2 of the Australian Standard and is being implemented (Standards Australia, 2019). This reflects adoption of similar visual-only inspection reforms in the European Union (EC, 2014).

Acknowledgements Supported in part by Australian Pork Limited and the Australian Meat Processors Corporation.

\section{References}

EC 2014. Commission Regulation EU No 219/2014 of 7 March 2014 amending Annex1 to Regulation (EC) No854/2004 of the European Parliament and of the Council. European Commission, Brussels, Belgium.

EFSA 2011. EFSA Journal. 9, 2351.

Hamilton DH, Gallas P, Lyall L, Lester S, McOrist S, Hathaway SC and Pointon AM 2002. Veterinary Record. 151, 110116.

Pointon A, Jackowiak J, Slade J and Paton M 2008. MLA Final Report A.SCC.0048. Meat \& Livestock Australia Ltd, North Sydney, NSW.

Pointon AM, Hamilton DH and Kiermeier A 2017. APL Final Report 2015/023. Australian Pork Ltd, Barton, ACT.

Pointon AM, Hamilton DH and Kiermeier A 2018. Food Control. 90, 222-232.

Standards Australia 2007. Food Regulation Standing Committee Technical Report Series 3. AS 4696:2007. Standards Australia, Sydney, NSW.

Standards Australia 2019. Amended AS 4696:2007. Standards Australia, Sydney, NSW. In Press.

Willeberg P, Wedam JM, Gardner IA, Holmes JC, Mousing J, Kyrval L, Enøe C, Andersen S and Leontides L 1997. Epidémiolgie et Santé Animale. 31/32, 04.20.1-04.20.3. 


\section{Pig risk-based review of post-mortem inspection of kidneys of pigs in Australia}

A M Pointon $^{1}$, D R Hamilton ${ }^{2}$ and A Kiermeier ${ }^{3}$

${ }^{1}$ APFoodIntegrity Pty Ltd, Australia, ${ }^{2}$ D Hamilton Consulting Pty Ltd, Australia, ${ }^{3}$ Statistical Process Improvement Consulting \& Training Pty Ltd, Australia

andypointon.food@iinet.com.au

Application Alternative post-mortem inspection procedures approved for pig kidneys provide equivalent wholesomeness for consumption and abattoir personnel with additional protection from an occupational zoonosis, while effectively monitoring animal health status and enabling enhanced processing efficiency.

Introduction Following approval of routine visual only inspection of pigs in the amended Australian Standard (Australian Standard, 2019), processors requested that further work be undertaken to validate the equivalence of observing unenucleated kidneys as an alternative inspection procedure to palpating enucleated kidneys (Pointon et al., 2017).

Materials and methods The effectiveness of alternative post-mortem inspection procedures for detecting gross abnormalites of pig kidneys affecting food safety, wholesomeness and animal health (including zoonoses) and welfare surveillance were quantified. A national snapshot survey of one week's production ( $\sim 85000$ pigs) through the seven export licenced abattoirs, covering approximately $85 \%$ of pigs slaughtered weekly, was conducted to estimate the prevalence rate and type of kidney gross abnormalities occurring. Carcasses were examined over one week/abattoir during late winter to encompass pigs from all herd sizes, production systems and regions on a proportional basis. Inspection was by palpation of enucleated kidneys (Standards Australia, 2007). Full methods are reported by Pointon et al. (2017).

Results The main findings of the snapshot survey include:

1. The prevalence of gross abnormalities of kidneys was $6.43 \%$ of 84047 pigs inspected;

2. Only $12(0.014 \%)$ of 84047 pigs had gross abnormalities of food safety significance $(1.4 / 10000$ carcasses $)$;

3. Of the 5403 pigs with gross abnormalities, $65.0 \%$ had cysts;

4. Nephritis was detected in $2.0 \%$ of 84047 pigs with $0.49 \%$ of 84047 pigs having Grade 2 nephritis, the latter being typified by a mottled whitish capsule, uneven surface and often being misshapen;

5. For pigs inspected by observation of unenucleated kidneys, the estimated increase in non-detection rates on a per 10000 carcass-basis was substantial for Grade 1 nephritis and moderate for Grade 2 nephritis and cysts;

6. In terms of significance for carcass disposition judgement, a total of 27 of 84047 inspected pigs with kidney gross abnormalities were totally condemned $(0.032 \%)$. Of these, five carcasses were condemned for food safety reasons, these being septicaemia (four) and pyaemia (one) (i.e. 0.6/10 000 carcasses). Another five carcasses were condemned for reasons unrelated to kidney lesions detected in that carcass, i.e. pigs condemned due to arthritis (four) and pyrexia (one) that had cysts;

7. All but one of the 27 pigs that were totally condemned with kidney lesions, had major evidence elsewhere in the carcass supporting total condemnation (e.g., uraemia, anaemia). The data supports that observing unenucleated kidneys has a negligible effect on determining final carcass disposition;

8. Metastasis of a kidney tumour was the probable reason for 1 of the 27 total carcass condemnations.

Conclusions The prevalence of gross kidney abnormalities of food safety significance is negligible. Inspection of unenucleated kidneys may substantially increase non-detection of some gross abnormalities e.g. mild nephritis and cysts. Gross abnormalities of kidneys are rarely of relevance for determining final carcass disposition judgement. Kidneys with Grade 2 nephritis have high specificity for leptospirosis (Chappel et al., 1992; Pointon et al., 2017). Observing unenucleated kidneys remains a useful surveillance tool for identifying herds likely to be infected with leptospirosis. Consequently, when kidneys are kept for human consumption the alternative recommended is to observe enucleated, rather than palpate. When not for human consumption, kidneys are to be left unenucleated for inspection by observation. These alternative procedures provide additional protection from an occupational zoonosis. This alternative procedure has been approved for the amendment of Schedule 2 of the Australian Standard and is being officially implemented (Standards Australia, 2019).

Acknowledgements Supported in part by Australian Pork Limited and the Australian Meat Processors Corporation. Abattoir personnel who collected the data are thanked for their assistance.

\section{References}

Chappel RJ, Prime RW, Millar BD, Mead LJ, Jones RT and Adler B 1992. Veterinary Microbiology. 30: $151-163$. Pointon A, Hamilton D and Kiermeier A 2017. APL Final Report 2017/2207. Australian Pork Ltd, Barton, ACT. Standards Australia 2007. Food Regulation Standing Committee Technical Report Series 3. AS 4696:2007. Standards Australia, Sydney, NSW.

Standards Australia 2019. Amended AS 4696:2007. Standards Australia, Sydney, NSW. In Press. 


\section{Antibiotic use and the expression of wild type patterns for Enterococcus species isolates: A comparison of three different pork production systems}

$\underline{\text { D L Turpin }}^{1,2}, \mathrm{~J} \mathrm{M} \mathrm{Pluske}^{3}$ and J R Pluske ${ }^{1}$

${ }^{1}$ Murdoch University, Australia, ${ }^{2}$ Pork Innovation Western Australia, Australia, ${ }^{3}$ SciEcons Consulting, Australia Diana.Turpin@murdoch.edu.au

Application This observational pilot study demonstrates a simple methodology for collecting antibiotic use data.

Introduction As pressure to record antibiotic use increases in the current climate, the Australian pork industry requires methods to benchmark antibiotic use and track resistance. Previously, surveillance projects in Australia have had a heavy focus on antimicrobial resistance (Abraham et al., 2017) with less focus on antimicrobial use, with the exception of Jordan et al. (2009) who collected information on patterns of antimicrobial use and frequency. However, actual amounts of antimicrobials used on farm were not reported. In the present study, methodology developed by Pipestone Veterinary Services (pipestonepart.com) was used to benchmark antibiotic use over a six-month period for three different production systems in Western Australia. The expression of antimicrobial wild type (i.e. as the bacterial species occurs in nature, without acquired or mutational resistance to a drug) patterns for Enterococcus spp. were then compared across farms to test the hypothesis that there is an association between the levels of antibiotics used on farm and wild type expression.

Material and methods The amounts of antibiotic medication used for in-feed, in-water and injectable treatments were collected from a small scale $(<500$ sows, Farm 1), large scale $(>1000$ sows, Farm 2$)$, and an outdoor production system (Farm 3) over a period of six months. Farms were selected by convenience sampling. In-feed data were collected from feed delivery dockets while data for in-water and injectable antibiotic medications were collected from purchase orders from suppliers. The usage metric was grams (g) per head with head representing the average throughput (market pigs) per month for each farm. Each of the three farms was then classified as least use (0-2 g/head, Farm 1), intermediate use (2-15 g/head, Farm 3), or most use (>15 g/head, Farm 2) based on their average antibiotic use over six months. Sixty faecal samples were collected from each farm covering all stages of production: farrowing, weaner, grower and finisher. Samples were sent to ACE Laboratory Services (East Bendigo, Australia) for Enterococcus spp. isolation and sensitivity testing. The minimum inhibitory concentrations (MIC) were interpreted according to the epidemiological cut off(ECOFF) values from The European Committee on Antimicrobial Susceptibility Testing guidelines (version 9.0, 2019 http://www.eucast.org/clinical_breakpoints/). Wild-type profiles were generated for each farm and a Chi-square analysis (IBM SPSS Statistics for Windows, v24.0. Armonk, NY) was used to determine if there was a difference in wild type expression for the Enterococcus spp. isolates between farms.

Results Within Enterococcus spp. isolates, the percentage of wild-type or non-wild type isolate expression for Ampicillin, Florfenicol, Gentamicin and Penicillin was different between the three farms (Table 1). However, this relationship was not necessarily associated with antibiotic use, with the outdoor farm (intermediate use) showing the highest level of nonwild type MICs (Figure 1). The antibiotics tested were part of the Sensititre ${ }^{\mathrm{TM}}$ BOPO7F AST Plate (Thermo Fisher Scientific, Waltham, MA, USA) and only antimicrobials with a determined ECOFF value are presented.

Table 1. Chi-square analysis between farms ${ }^{1}$ for percentage of wild type expression

\begin{tabular}{|c|c|c|}
\hline Antibiotic & $\chi^{2}(2)$ & Significance \\
\hline Ampicillin & 107.3 & $<0.001$ \\
\hline Florfenicol & 69.3 & $<0.001$ \\
\hline Gentamicin & 133.3 & $<0.001$ \\
\hline Penicillin & 198.0 & $<0.001$ \\
\hline
\end{tabular}

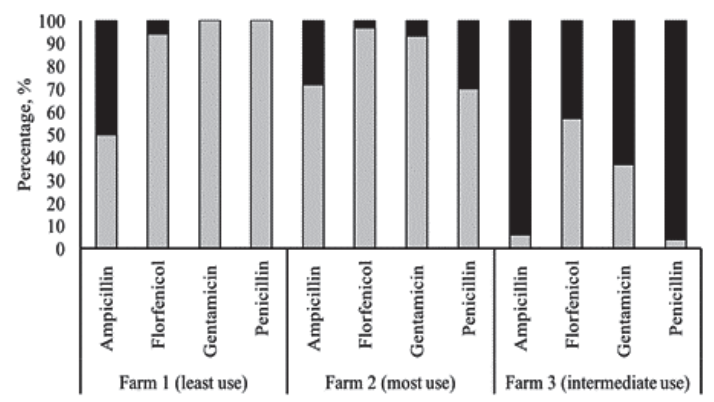

Figure 1. Antimicrobial wild-type pattern ( $\square$ wildtype \%; non-wild type \%) for Enterococcus species.

Conclusion There was no obvious relationship between antibiotic use and the expression of non-wild type MICS for Entertococcus spp. for the selected antibiotics; however, it must be acknowledged that other factors that play a role in the development of antimicrobial resistance were not explored.

Acknowledgements Supported in part by the Grower Group R\&D grants program, WA Department of Primary Industries and Regional Development's Agribusiness Innovation Fund. Staff at Murdoch University are thanked for technical assistance.

\section{References}

Jordan D, Chin JC, Fahy V, Barton MD, Smith M and Trott D 2009. Australian Veterinary Journal. 87, $222-229$.

Abraham S, O’Dea M, Page S and Trott D 2017. Animal Production Science. 57, 2398-2407. 


\title{
Impact of water quality on the solubility of veterinary antibiotics administered to pigs through drinking water
}

\author{
L J Edwards $^{1}$, S B Little ${ }^{2}$, A Watt ${ }^{2}$ and H Billman-Jacobe ${ }^{2}$ \\ ${ }^{1}$ Ridley AgriProducts Pty Ltd, Australia, ${ }^{2}$ The University of Melbourne, Australia \\ Louise.Edwards@ridley.com.au
}

\begin{abstract}
Application The results of this study indicate that the solubility of antibiotics varies across different water sources, and good quality water is essential to ensure appropriate antibiotic use fitting with antimicrobial stewardship.
\end{abstract}

Introduction Water is an essential nutrient for optimal animal performance, health and welfare. Despite its importance as the first nutrient in animal nutrition, a recent study (Edwards, 2018) identified that water management was often overlooked with water quality being sub-optimal for one or more quality parameters across all farms surveyed. Of note was the observation that $75 \%$ were utilising water as a means to administer water-soluble additives, including veterinary antibiotics. This observation is of concern when considering that the solubility, stability and antibacterial activity of some antibiotics can be affected by water quality parameters such as $\mathrm{pH}$ or high mineral levels (Dorr et al., 2009; Lumb et al., 2017). Given the importance of antimicrobial stewardship, the objectives of this study were to understand the impact of water quality on the solubility, stability and antibacterial activity of commonly used water-soluble antibiotics.

Material and methods Using the findings of Edwards (2018), seven commonly used water-soluble antibiotics, each with a different active constituent, were sourced and a range of properties including solubility, particle size and antibacterial activity evaluated. Water samples of differing qualities (ultrapure, bore, dam and river) were used to test the solubility of each product over a 24 hour period. Solutions were prepared to the concentration to be consumed by pigs at the drinker, or as a stock solution, at 100 times the drinker concentration, prepared for administration into the drinking water line through a proportional dosing pump.

Results When prepared at drinker concentration in ultrapure water, all antibiotics were in solution within 120 seconds and no insoluble particles were visible at 24 hours (Table 1). In contrast, when the stock solution was prepared in ultrapure water, insoluble particles were observed with four of the products tested immediately and at 24 hours. When prepared at drinker concentration in bore and river water, all products were in solution within 120 seconds with minimal precipitation observed at 24 hours. Sediment accumulation was observed in all the solutions prepared at drinker concentration in dam water, except for one antibiotic. Preliminary results indicated that all antibiotic products demonstrated antibacterial activity at the concentration supplied however, activity was variable when prepared as a 10 times dilution or when fractionated into different particle sizes. These observations warrant further investigation.

Table 1. Solubility of veterinary antibiotics when dissolved at drinker concentration in different water types, sediment accumulation at 24 hours indicated by bold type.

\begin{tabular}{lccccccc}
\hline & $\mathrm{A}$ & $\mathrm{L}$ & $\mathrm{S} / \mathrm{T}$ & $\mathrm{OTC}$ & $\mathrm{CTC}$ & $\mathrm{L} / \mathrm{S}$ & $\mathrm{T}$ \\
\hline Concentration at drinker $(\mathrm{mg} / \mathrm{ml})$ & 0.123 & 0.042 & 0.685 & 0.331 & 0.253 & 0.096 & 0.250 \\
Time (s) to dissolve at drinker concentration & & & & & & & \\
$\quad$ & $120^{*}$ & $<10$ & $20^{*}$ & 20 & 45 & $<10$ & $<10$ \\
Ultrapure & $\mathbf{1 2 0 *}$ & $<10$ & $\mathbf{2 0 *}$ & 20 & 120 & 20 & 15 \\
Bore & $120^{*}$ & $<10$ & $\mathbf{3 0}$ & $\mathbf{3 0}$ & $\mathbf{1 8 0}$ & $\mathbf{2 5}$ & $\mathbf{1 5}$ \\
Dam & $120^{*}$ & $<10$ & 20 & $\mathbf{3 0}$ & 120 & 15 & $<10$ \\
River &
\end{tabular}

*very fine particles present; A, Amoxycillin trihydrate $(870 \mathrm{~g} / \mathrm{kg})$; L, Lincospectin base ( $\geq 790)$; S/T, Sulfadiazine/ Trimethoprim (400/80), OTC, Oxytetracycline hydrochloride (880), CTC, Chlortetracycline hydrochloride (950), L/S, Lincomycin hydrochloride monohydrate/Spectinomycin sulphate tetrahydrate (222/445), T, Tylosin tartrate ( $\geq 800)$.

Conclusion This study has demonstrated that the solubility and stability of several commercially available water-soluble antibiotics may be compromised in the field particularly when being prepared as stock solutions or at concentrations in excess of their solubility. However, even when prepared at concentrations below saturation point (drinker concentration) the solubility of antibiotics was observed to be impacted by water quality. It is recommended that producers and veterinarians routinely monitor water quality, in particular $\mathrm{pH}$ and iron, to enable on-farm decision-making to support the responsible use of antibiotics, the health, welfare of their animals and in turn the economics of their business.

Acknowledgements Supported in part by Pork CRC Limited and Ridley AgriProducts Pty Ltd.

\section{References}

Dorr PM, Madson D, Wayne S, Scheidt AB and Almond GW 2009. Journal of Swine Health and Production. 17, 217 -

222.

Edwards LJ 2018. Pork CRC Final Report 2A-118. Pork CRC Ltd, Roseworthy SA.

Lumb KR, Robertson J, Scott HE and Woolfenden NJ 2017. AHDB, Stoneleigh Park, Warwickshire, UK. https://pork.ahdb.org.uk/media/274247/51510014_ft014_raft-solutions-ltd-water-report_approved_september2017.pdf 


\section{Competitive exclusion by promoting growth of beneficial bacteria after Salmonella infection}

N de Groot ${ }^{1}$, L Faba $^{2}$ and P Roubos-van der Hil ${ }^{2}$

${ }^{1}$ Trouw Nutrition Innovation, the Netherlands, ${ }^{2}$ Trouw Nutrition $R \& D$, the Netherlands nienke.de.groot@trouwnutrition.com

Application Fungal fermented products and organic acids benefit the intestinal microbiota of piglets. The prebiotic effect included a reduction of the faecal excretion of pathogenic and cefotaxime resistant bacteria during a Salmonella challenge.

Introduction Fungal fermented products and their derivatives contain several compounds that may play a role in gut health by improving the microbiota balance. Indigestible oligo- and disaccharides from fungal fermentation of feed material can bind and reduce intestinal pathogens such as Salmonella typhimurium or E.coli and exhibit a prebiotic effect (Agunos et al., 2007). In addition, fungal cell wall components including beta-glucans may reveal prebiotic properties supporting intestinal health (Kim et al., 2019). Organic acids are known to increase stomach barrier function by reducing stomach $\mathrm{pH}$ and destabilize bacterial membrane function resulting in bacterial cell death (Zentek et al., 2013). Combining additive strategies can be a valuable option to prevent colonisation and transmission of infectious bacteria in herds. This study evaluates a combined feed additive approach to prevent specific Enterobacteriacae (Salmonella and E.coli) problems and a prebiotic effect on other bacterial families after infection with Salmonella enterica serovar Typhimurium (Salmonella typhimurium) in piglets.

Material and methods Twenty-four individually housed weaned piglets consumed either a control diet or the same diet supplemented with organic acids for antibacterial properties and a novel feed additive (FA) blend, containing mannanase hydrolysed copra meal and rye overgrown with mycelium of Agaricus subrufescens. Each piglet received a feed matrix containing $10^{9} \mathrm{CFU}$ Salmonella typhimurium daily from $5 \mathrm{~d}$ after weaning until d 12. Salmonella and ESBL/AmpCproducing Escherichia coli (ESBL/AmpC) faecal shedding was determined. On d 8 and 13 of the study $1 \mathrm{~g}$ faeces per faecal sample was used for microbial sequencing using 16S RNA sequencing. Comparisons were made between microbial composition of faeces on d 8 compared to d 13 and between treatment and control group. Linear Discriminant Analysis (LDA) model was used to calculate relative difference among bacteria genera.

Results Infection was successful and Salmonella peak of shedding (d 8) was lower $(P=0.01)$ for treated pigs (4.8 log $\mathrm{CFU} / \mathrm{g}$ ) than the control (5.8 log CFU/g). Similarly, shedding was lower $(P=0.01)$ overall infection week $(4.0 \mathrm{log} \mathrm{CFU} / \mathrm{g}$ vs $5.1 \log \mathrm{CFU} / \mathrm{g})$. Interestingly, dietary treatment also reduced ESBL/AmpC shedding $(0.5 \log \mathrm{CFU} / \mathrm{g} v s 2.0 \mathrm{log} \mathrm{CFU} / \mathrm{g}$; $P=0.01$ ) during the one week challenge with Salmonella typhimurium, yet this effect disappeared post challenge (Figure 1). Regardless of treatments, microbiota analysis showed lower Lactobacillus levels at d 8 compared to d 13 (23.2\% and $44.0 \%$, respectively), while Clostridium levels were higher at d 8 compared to d 13 (17.5\% and 9.86\%, respectively). Comparing dietary treatments, levels of Streptococcus were lower in animals receiving FA at d 8 (1.9\% and $0.09 \%$, respectively). At d 13, Lactobacillus levels were higher in the faeces of piglets receiving the FA treatment compared to the control group, while Clostridia spp. levels were higher in the control group (Figure 2).

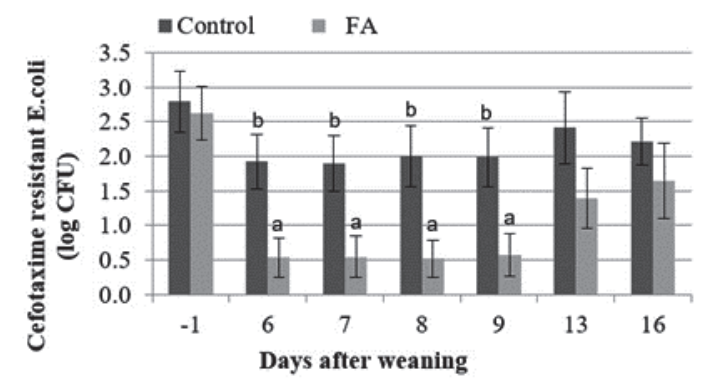

Figure 1. ESBL/AmpC shedding after challenge with Salmonella at $5 \mathrm{~d}$ post weaning

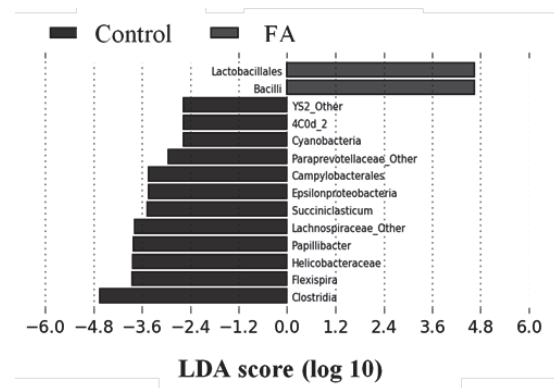

Figure 2. Comparison between control and FA treatment on $\mathrm{d} 13$

Conclusion The feed additive treatment showed a reduction in shedding of Salmonella typhimurium and cefotaxime resistant (AMR) E. coli shedding, indicating a possible benefit for reducing antimicrobials in weaned piglets. The lower level of Lactobacillus and the higher level of Clostridium at d 8 may be a sign of impaired intestinal health during Salmonella infection as these changes are considered as a measurement to determine gut health (Dou et al., 2017). It can be concluded that the FA blend acts as a microbiota modifier, with a higher abundance of Lactobacillus and a lower abundance of Clostridium spp. after a Salmonella challenge.

\section{References}

Agunos A, Ibuki M, Yokomizo F and Mine Y 2007. British Poultry Science. 48, 331-341.

Kim K, Ehrlich A, Perng V, Chase JA, Raybould H, Li X, Atwill ER, Whelan R, Sokale A and Liu Y 2019. Animal Feed Science and Technology 248,114-125.

Dou S, Gadonna-Widehem P, Rome V, Hamoudi D, Rhazi L, Lakhal L, Larcher T, Bahi-Jaber N, Pinon-Quintan A, Guyonvarch A, Huërou-Luron ILE and Abdennebi-Najar L 2017. PLOS ONE 12(1) e0169851.

Zentek J, Ferrara F, Pieper R, Tedin L, Meyer W and Vahjen W 2013. Journal of Animal Science. 91, 3200-3210. 


\section{Organic acids in drinking water improve water intake and performance of nursery piglets - a meta-analysis}

$\mathrm{N} \mathrm{de} \mathrm{Groot}^{1}, \mathrm{G} \mathrm{Heim}^{1}$ and N F Ferguson ${ }^{2}$

${ }^{1}$ Trouw Nutrition Innovation, the Netherlands, ${ }^{2}$ Trouw Nutrition R\&D, Canada

nienke.de.groot@trouwnutrition.com

Application Free and buffered organic acids in drinking water of weaned piglets increase water and feed intake and reduce feed conversion, therefore increasing economic performance.

Introduction The use of organic acids in feed or water in improving piglet performance in the first weeks after weaning has been well established (Partanen et al., 1999). After weaning the hydrochloric acid production in the stomach of piglets is not yet sufficient for proper digestion. The use of organic acids to decrease $\mathrm{pH}$ in the stomach will result in increased activity of proteolytic enzymes (Kim et al., 2004). Several nursery studies were conducted, and their data combined into a meta-analysis to compare the effect of organic acid blends, via drinking water, with the hypothesis that inclusion of organic acids will improve the performance of piglets post-weaning.

Material and methods Six commercial studies with a total of 4368 weaned piglets (age 18-21 d, $6.17 \pm 0.48 \mathrm{~kg}$ ) were included, with each study consisting of two groups: a Control (no drinking water acidifier) and a drinking water additive (DWA) based on free and buffered organic acids (1-2 L/1000 L water) applied for the first 3 weeks post-weaning. The inclusion rate was determined based on water titration, to attain the recommended water $\mathrm{pH}$ of 3.8 . Water and feed were provided ad libitum throughout the study period. In each study, control and treatment diets were similar; three phase diets with low zinc (100-300 ppm), a range of copper (25-150 ppm) and no antibiotic inclusion. Piglets were monitored on body weight (BW), average daily gain (ADG), average daily feed intake (ADFI), average daily water intake (ADWI) (as disappearance) and feed conversion ratio (FCR), during the $42 \mathrm{~d}$ trial period. Based on raw data from each study, a metaanalysis was done, the performance was analysed using an ANOVA. Data of two studies were analysed for body weight variation, since not all individual body weights were taken in other studies.

Results The results showed that piglets receiving a drinking water acidifier had significantly higher ADWI in the first three weeks post-weaning (Figure 1). Treatment significantly increased BW, ADG and ADFI, and significantly reduced FCR compared to control during the whole nursery period (Table 1). In addition, the DWA treatment tended to reduce body weight variation within pen: there was a tendency to have fewer light piglets and more heavy piglets (Figure 2).

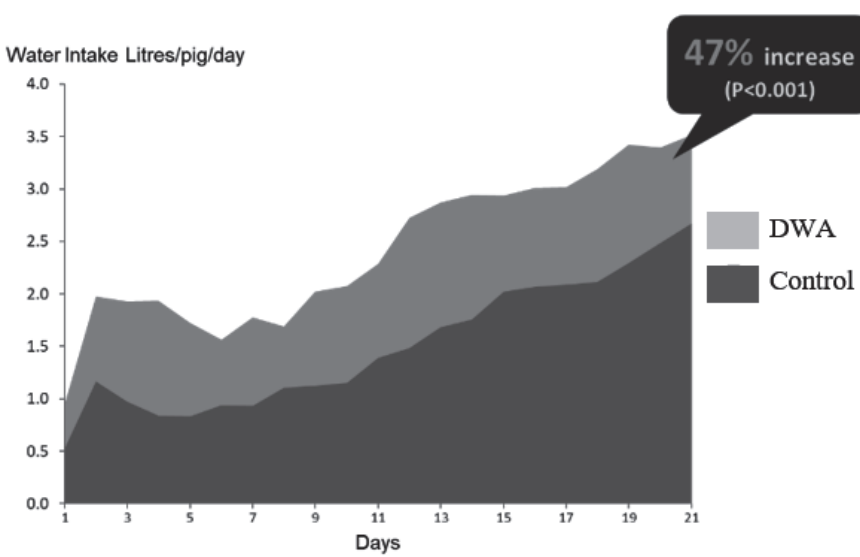

Figure 1. ADWI of nursery piglets during the first three weeks post-weaning, meta-analysis of six studies $(P<$ $0.001)$.
Table 1. Performance of nursery piglets when comparing organic acid blend in water (DWA) vs control after 42 days, meta-analysis of six studies.

\begin{tabular}{lccc}
\hline Treatment & Control & DWA & $P$ value \\
\hline Final weight $(\mathrm{kg})$ & 24.5 & 25.0 & $<0.001$ \\
ADG $(\mathrm{g} / \mathrm{d})$ & 436 & 448 & $<0.001$ \\
ADFI $(\mathrm{g} / \mathrm{d})$ & 602 & 611 & 0.040 \\
FCR $(\mathrm{g} / \mathrm{g})$ & 1.38 & 1.37 & $<0.001$ \\
\hline
\end{tabular}

ADG, average daily gain; ADFI, average daily feed intake; FCR, feed conversion ratio

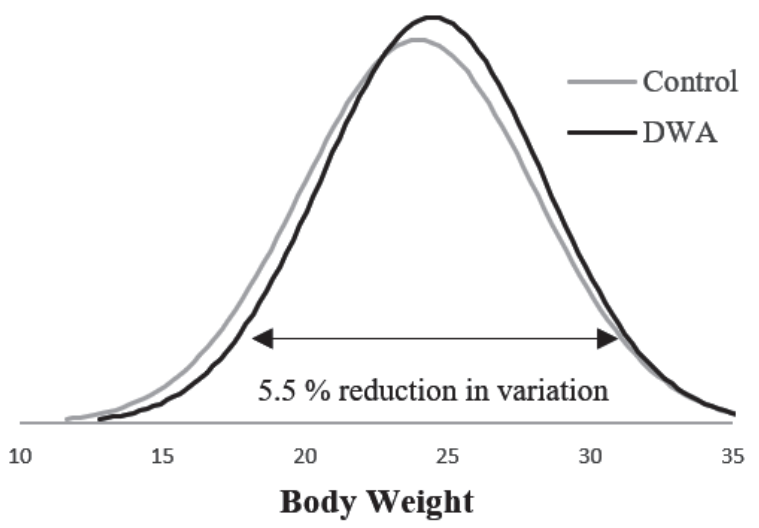

Figure 2. BW variations within pen (final BW 6 weeks post-weaning), control vs DWA $(P=0.08)$.

Data from two similar trials conducted at two different locations.

Conclusion The stress of weaning can negatively affect feed intake, and low feed intake can impair growth performance in nursery pigs. Thus, it is important to increase feed intake in newly weaned pigs in order to improve growth. Immediately after weaning, pigs are less prone to eating dry food and compensate by drinking water. This trial shows that the use of the drinking water acidifier increases feed efficiency, resulting in improved performance.

\section{References}

Kim YY, Kil DY, Oh HK and Han IK 2004. Asian-Australian Journal of Animal Science. 18, 1048-1060.

Partanen KH and Mroz Z 1999. Nutrition Research Reviews. 12, 117-145. 


\section{Impact of dietary fibre type and content on Escherichia coli shedding and microbial structure after weaning}

$\underline{\text { S N Jenkins }}^{1}$, I S Waite ${ }^{1}$, J P Mansfield ${ }^{2}, \mathrm{~J} \mathrm{C} \mathrm{Kim}^{2,3}$ and J R Pluske ${ }^{2}$

${ }^{1}$ The University of Western Australia, Australia, ${ }^{2}$ Murdoch University, Australia, ${ }^{3}$ AB Vista, Inc., UK

sasha.jenkins@uwa.edu.au

Application Piggeries routinely use in-feed antimicrobials to reduce post-weaning diarrhoea (PWD) but their widespread use has increased antimicrobial resistance (AR) within herds. Identifying better post-weaning management will lead to a reduction in antimicrobial use and improved animal welfare.

Introduction Increasing insoluble non-starch polysaccharides (iNSP) in the diets of weaner pigs has been shown to improve the gastrointestinal tract (GIT) function and reduce ETEC infection, but the mechanisms involved are not fully understood (Pluske et al., 2002). One possibility is that diets rich in iNSP promote the growth of short chain fatty acid producing bacteria (SCFAB) whilst supressing the proliferation of amide producing bacteria (AB) making the GIT environment less favourable for ETEC infection (Chen et al., 2016). It was hypothesised that pigs fed iNSP would have a higher abundance of SCFAB and a lower abundance of AB resulting in less ETEC shedding.

Materials and methods An experiment having a $2 \times 2 \times 4$ factorial arrangement of treatments using 96 individuallyhoused male weaner pigs (initial body weight $8.8 \pm 0.05 \mathrm{~kg}$ ) was conducted for 2 weeks, with factors being with and without ETEC challenge, low and high sNSP (7 versus $28 \mathrm{~g}$ soluble arabinoxylan NSP/kg) and four levels of iNSP added as Opticell ${ }^{\circledR}$ (Agromed Austria GmbH, Kregsmünster, Austria) equivalent to 5.5, 19.0, 34.5 and $51 \mathrm{~g}$ iNSP/kg. Half the pigs were orally infected with an ETEC strain (0149:K91:F4) on d 5. Blood samples were collected pre and post infection to compare the haptoglobin, plasma urea and white blood cell counts levels. Faecal samples were collected $9 \mathrm{~d}$ after infection with ETEC. Faecal $\beta$-haemolytic E. coli shedding was measured. Extracted faecal DNA was quantified and PCR-amplified using universal 16S rRNA primers V4/5 (515F and 806R) prior to sequencing. Sequence data were analysed using the QIIME pipeline and the relationship between dietary fibre, E. coli shedding and microbial diversity was assessed using canonical correspondence analysis (CCA) approaches.

Results Overall, the GIT microbiota composition of the piglets is strongly influenced by dietary fibre and ETEC-challenge (Figure 1). Increasing dietary iNSP (top two quadrants) resulted in reduced E.coli shedding (Jenkins et al., 2015) and a GIT microbiota dominated by butyrate-producing bacteria (Christensenella, Roseburia, Coprococcus; \#16, \#20, \#22) and lactate-producing bacteria (Lactobacillus, Ruminococcus, Enterococcus; \#2, \#12, \#17). In contrast, increasing dietary sNSP (bottom two quadrants) was associated with E.coli shedding and increased abundance of AB (Peptococcus, Peptostreptococcus; \#14, \#21).

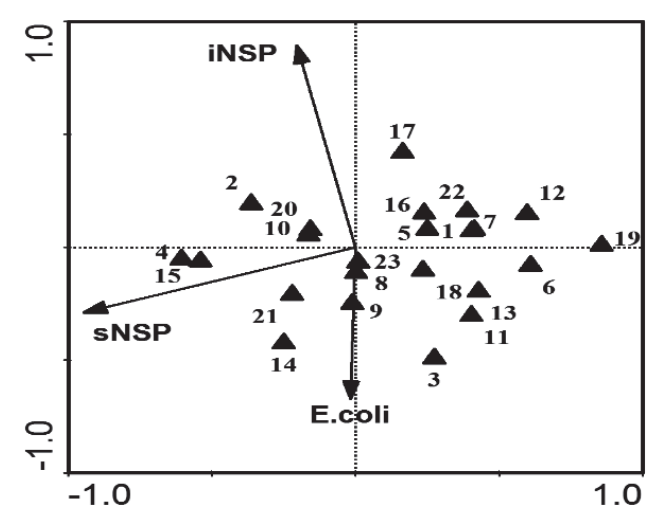

Figure 1. The CCA biplot showing the influence of fibre type (iNSP; sNSP) and E.coli shedding on the faecal microbial community composition (key individual taxa distributions \#1-22)

Conclusion These results from increasing iNSP support previous studies where the incidence of diarrhoea was greatly reduced in ETEC-challenged weaned piglets that received dietary supplementation of probiotics containing Enterococcus and Lactobacillus (Chen et al., 2016). In contrast, increasing sNSP, resulted in toxic substrates that irritate the epithelium and increase ETEC infection resulting in higher rates of post-weaning diarrhoea (Chen et al., 2016; Pluske et al., 2002). Increasing iNSP content in the diet promoted favourable GIT microbiota that in turn reduced the proliferation of amide producing bacteria and ETEC infection.

Acknowledgements Supported in part by Pork CRC Limited.

\section{References}

Chen HS, Velayudhan DE, Li A, Feng Z, Liu D, Yin YL and Nyachoti CM 2016. Canadian Journal of Animal Science. 96, 609-618.

Jenkins SN, Waite IS, Mansfield J, Kim JC, Pluske JR 2015. Animal Production Science 55, 1451-1451.

Pluske JR, Pethick DW, Hopwood DE and Hampson DJ 2002. Nutrition Research Reviews. 15, 333-371. 


\section{Xylo-oligosaccharide and xylanase improved growth performance in weaner pigs fed an antibiotic free low $\mathrm{ZnO}$ diet when exposed to an unsanitary environment}

H M Cho ${ }^{1}$, S S Wickramasuriya ${ }^{1}$, S P Macelline ${ }^{1}$, J S Hong ${ }^{1}$, Y B Kim ${ }^{1}$ J C Kim², G Cordero², M R Bedford ${ }^{2}$ and J M $\underline{\mathrm{Heo}}^{1}$

${ }^{1}$ Chungnam National University, Republic of Korea. ${ }^{2} A B$ Vista, UK

jmheo@cnu.ac.kr

Application Xylo-oligosaccharide and xylanase supplementation in the diet of weaner pigs can be used as a strategy to improve performance in piglets fed an antibiotic-free low zinc oxide diet.

Introduction Legislative pressures are increasing to reduce the use of antibiotics and zinc oxide $(\mathrm{ZnO})$ which are used for stabilisation of intestinal microbiota. Xylo-oligosaccharide (XOS) supplementation has been shown to improve growth performance and reduce post-weaning diarrhoea, suggesting improved activity of beneficial bacteria such as Bifidobacterium in the large intestine (Yang et al., 2015). Xylanase supplementation is also known to increase in vivo production of XOS hence supplementing a xylanase with XOS can accelerate the beneficial effect of XOS (Bedford et al., 2018). The hypothesis tested was to determine if the combined supplementation of XOS and xylanase can reduce post-weaning diarrhoea and improve performance in piglets fed an antibiotic-free low $\mathrm{ZnO}$ diet compared to fructooligosaccharide (FOS) and mannan-oligosaccharide (MOS) when housed in a poorer sanitary environment.

Materials and methods A total of 144 male pigs [Duroc x (Yorkshire x Landrace)] weaned at $28 \mathrm{~d}$ of age with initial body weight $(\mathrm{BW})$ of $7.5 \pm 0.7 \mathrm{~kg}$ (mean $\pm \mathrm{SEM}$ ) were randomly allocated to six dietary treatments [Sanitary condition: control (CON; < 150 ppm ZnO), CON with XOS plus xylanases (CXX; Signis, AB Vista, Marlborough, UK; 100 g/t); Poorer sanitary condition (without cleaning/disinfection of a previously populated room) : CON, CXX, CON with MOS (CM; Bio-Mos ${ }^{\circledR}$, Alltech Inc, Nicholasville, KY, USA; 1000 g/t), CON with FOS (CF; FOS-MAX ${ }^{\circledR}$, Dreamfeed Inc., Seoul, Republic of Korea; $2000 \mathrm{~g} / \mathrm{t}$ )] to give 6 replicates per treatment with 4 pigs per pen. All diets were formulated based on corn, wheat and soybean meal to meet NRC nutrient specification (NRC, 2012). Average daily gain (ADG) and average daily feed intake (ADFI) were measured for 6 weeks. The incidence of diarrhoea, expressed as proportion of days with diarrhoea, was measured for the first $14 \mathrm{~d}$ (i.e., pen basis). Data were analysed as a completely randomized block design using general linear model procedure of ANOVA for environmental effect and diet effect under poorer sanitary condition.

Results Housing piglets under poorer sanitary conditions reduced ADG by $12 \%(P<0.001)$ and increased incidence of diarrhoea by $33 \%(P<0.05)$. Supplementation of CXX increased ADG regardless of housing condition but the improvement was more notable under unsanitary conditions $(8 \%$ and $15 \%$ improvement under sanitary and unsanitary condition, respectively; $P<0.001$ ). Under unsanitary conditions, supplementation of FOS and MOS did not improve $\operatorname{ADG}(P>0.05)$.

Table 1. Impact of sanitary conditions and dietary treatments (control, CON; control with xylo-oligosaccharide plus

xylanases, CXX; control with mannan-oligosaccharides, CM; control with fructo-oligosaccharides, CF) on growth performance (average daily gain, ADG; average daily feed intake, ADFI; feed conversion ratio, FCR) over 42 days and the incidence of diarrhoea (DI) at day 14 after weaning.

\begin{tabular}{lcccccccccc}
\hline & \multicolumn{3}{c}{ Sanitary } & \multicolumn{4}{c}{ Poorer Sanitary } & \multicolumn{2}{c}{ SEM } & \multicolumn{2}{c}{$P$ value } \\
& CON & CXX & CON & CXX & CM & CF & & D & DPS \\
\hline ADG $(\mathrm{g} / \mathrm{d})$ & $343^{\mathrm{bc}}$ & $370^{\mathrm{a}}$ & $302^{\mathrm{d}}$ & $348^{\mathrm{ab}}{ }_{\mathrm{x}}$ & $319^{\mathrm{cd}}$ & $321^{\mathrm{cd}} \mathrm{y}$ & 3.5 & $<0.001$ & $<0.001$ & $<0.001$ \\
ADFI $(\mathrm{g} / \mathrm{d})$ & 508 & 514 & 493 & 490 & 477 & 483 & 11.8 & 0.392 & 0.837 & 0.957 \\
FCR $(\mathrm{g} / \mathrm{g})$ & 1.81 & 1.72 & 1.85 & 1.66 & 1.66 & 1.69 & 0.038 & 0.907 & 0.305 & 0.210 \\
DI $\%)$ & 5.4 & 4.0 & 13.4 & 8.1 & 7.9 & 6.5 & 1.54 & 0.033 & 0.247 & 0.416 \\
\hline
\end{tabular}

$\overline{\mathrm{a}, \mathrm{b}, \mathrm{c}, \mathrm{d}}$ Means within a row with different superscripts differ significantly in dietary treatments $(P<0.05) ;{ }^{\mathrm{x}, \mathrm{y}, \mathrm{Z}}$ Means within a row with different subscripts differ in diets under poorer sanitary $(P<0.05)$; SEM, standard error of mean (for poorer sanitary); E, environment; D, diet; DPS, diet effect under poorer sanitary; DI, Diarrhoea index, percentage of days with diarrhoea with respect to total number of days; E x D interactions were not significant $(P>0.05)$.

Conclusion This study clearly demonstrated that housing piglets under unsanitary conditions has a negative effect on growth and diarrhoea score. Supplementation of CXX, which could stimulate arabinoxylan utilizing microbiota, can be a strategy to reduce the negative impact of high microbial loads in commercial production systems.

Acknowledgements This study was financially supported by AB Vista (Marlborough, UK)

\section{References}

Bedford MR 2018. British Poultry Science. 59, 486-493.

NRC 2012. Nutrient Requirements of Swine: $11^{\text {th }}$ Revised Edition. The National Academies Press, Washington DC, USA. Yang J, Summanen PH, Henning SM, Hsu M, Lam H, Huang J, Tseng C-H, Dowd SE, Finegold SM, Heber D and Li Z 2015. Frontiers in Physiology. 6, 216. 


\section{Feeding larger diameter pellets to piglets in lactation does not improve the weaning weight of piglets born to gilts but decreases the removal rate of pigs in the post-weaning period}

J R Pluske $^{1}$, J C Kim ${ }^{1,3}$, J R Craig 2 , R J Smits ${ }^{2}$, C J Braden ${ }^{2}$ and C J Brewster ${ }^{2}$

${ }^{1}$ Murdoch University, Australia, ${ }^{2}$ Rivalea Australia Pty Ltd, Australia ${ }^{3}$ AB Vista, UK

J.Pluske@murdoch.edu.au

Application A larger pellet diameter offered to piglets in lactation decreased post-weaning removal rate and improved performance.

Introduction Creep feed intake of piglets during lactation is positively related to a greater interest in the feed after weaning and subsequent feed intake and performance (Bruininx et al., 2002; Pluske et al., 2007). Nevertheless, consumption of creep feed in lactation is typically low and varies considerably between and within litters. Most efforts at increasing creep feed intake in lactation have focused on feed composition, with relatively less attention paid to physical characteristics of the feed offered to piglets. Commercial pellet sizes for piglet creep feed are typically $4 \mathrm{~mm}$ or less in line with the general thought that this size is better for chewing and swallowing and hence intake; however, there is evidence suggesting that young pigs are adaptable to a variety of pellet diameters both before and after weaning (Edge et al., 2005; van den Brand et al., 2014; Clark et al., 2016). Feeding large pellets, to $12 \mathrm{~mm}$ diameter, can increase feed intake of pigs during lactation and (or) in the first week in the nursery compared to feeding small pellets (van den Brand et al., 2014; Clark et al., 2016). This could be beneficial to piglets born to first-litter sows that have a lighter birth weight. Moreover, increased feed intake after weaning irrespective of parity might reduce the number of pigs removed from the production flow. This experiment examined these two propositions.

Material and methods A total of 240 mixed-parity sows (Large White x Landrace $\mathrm{F}_{1}$; Primegro ${ }^{\mathrm{TM}}$ Genetics, Corowa, NSW) were used in the study, in two replicates. Sows were randomly allocated upon entry to the farrowing sheds according to a 2 x 2 factorial arrangement of treatments, with the factors being (1) gilts vs sows (parity 2-4) and (2) a 4 $\mathrm{mm}$ diameter pellet of $4 \mathrm{~mm}$ length $v s$ a $9 \mathrm{~mm}$ diameter pellet of $12 \mathrm{~mm}$ length. Diets contained $\sim 15 \mathrm{MJ}$ digestible energy (DE) $/ \mathrm{kg}$ and $0.87 \mathrm{~g}$ SID lysine/MJ DE. Pellets were offered on an ad libitum basis from $\mathrm{d} 3$ of lactation to weaning at $\sim 26$ $\mathrm{d}$ of age; disappearance was recorded. After weaning, 2070 pigs born to the gilts and sows were mixed, divided into males and females, and placed into pens $(n=115)$ each of 18 pigs. Pigs offered the $4 \mathrm{~mm}$ diameter pellet or the $9 \mathrm{~mm}$ diameter pellet in lactation were penned together, enabling a comparison of pellet diameter on post-weaning production. Pigs were offered the same commercial diets (4 mm pellet diameter) ad libitum for $21 \mathrm{~d}$ after weaning. Data were analysed using the MIXED procedure of SPSS (IBM SPSS Statistics for Windows, v25.0. Armonk, NY), with litter and pen as experimental units. Chi-square analysis (SPSS) examined the percentage of pigs removed (mortalities and ill-thrift) and medicated after weaning.

Results Total creep feed disappearance in lactation was higher $(P<0.001)$ in litters offered the larger diameter pellet, but this did not translate to an improvement in litter weaning weight for piglets born to gilts (interaction, $P>0.05$ ). Piglets born to gilts were weaned lighter $(P<0.001)$ than piglets born to sows. In the post-weaning period, male and female pigs offered the larger diameter pellet during lactation showed tendencies to be removed at both a lower rate $(2.6 v s 3.9 \%, P$ $=0.11)$ and receive less medications $(3.3 \mathrm{vs} 4.9 \%, P=0.071)$ than pigs offered the smaller diameter pellet during the lactation period. Growth rate and feed intake after weaning were both increased (232 vs $210 \mathrm{~g} / \mathrm{d}, P=0.002 ; 293$ vs 270 $\mathrm{g} / \mathrm{d} ; P=0.009$, respectively) in pigs offered the larger diameter pellet during lactation.

Conclusion Offering piglets a larger diameter pellet in lactation did not improve the weaning weight of piglets born to gilts, but offered benefits to pigs in the post-weaning period in terms of a lowered removal rate and improved performance. This might be attributable to increased feed-related exploratory behavior as evidenced by the greater disappearance of creep feed in lactation.

Acknowledgements Supported in part by Australian Pork Limited. Appreciation is extended to the Research and Innovation Team at Rivalea Australia Pty Ltd.

\section{References}

Bruininx EMAM, Binnendijk GP, van der Peet-Schwering CMC, Schrama JW, den Hartog LA, Evers H and Beynen AC 2002. Journal of Animal Science. 80, 1413-1418.

Clark AB, De Jong JA, DeRouchey JM, Tokach MD, Dritz SS, Goodband RD and Woodworth JC 2016. Journal of Animal Science. 94(Suppl. 2), 100-101.

Edge HL, Dalby JA, Rowlinson P and Varley MA 2005. Livestock Production Science. 97, 203-209.

Pluske JR, Kim J-C, Hansen CF, Mullan BP, Payne HG, Hampson DJ, Callesen J and Wilson RH 2007. Archives of Animal Nutrition. 61, 469-480.

van den Brand H, Wamsteeker D, Oostindjer M, van Enckevort LCM, van der Poel AFB, Kemp B and Bolhuis JE 2014. Journal of Animal Science. 92, 4145-4153. 


\section{Phytogenic feed additives and dietary energy density can be used to improve the growth performance of pigs between 20 and $95 \mathrm{~kg}$ live weight.}

J Walker $^{1}$, D J Henman ${ }^{1}$, S L Beer ${ }^{1}$, D Meaney ${ }^{2}$ and N Gannon ${ }^{2}$

${ }^{1}$ Rivalea Australia Pty Ltd, Australia, ${ }^{2}$ Biomin Singapore Pte Ltd, Singapore.

dhenman@rivalea.com.au

Application Addition of the phytogenic feed additive, Digestarom ${ }^{\circledR}$ and manipulating dietary digestible energy (DE) levels and associated amino acid ratios can be used to improve the growth performance of grower/finisher pigs. The use of these strategies will depend on the relative cost of DE, amino acids and the phytogenic feed additive.

Introduction The marginal cost of digestible energy (DE) typically costs between $\$ 15$ and 20 per MJ in grower/finisher diets. Digestarom ${ }^{\circledR}$ (DIG; Biomin Animal Nutrition GmbH, Getzersdorf, Austria) is a phytogenic feed additive of plant origin used in swine nutrition to support gut performance and improve feed intake and feed conversion efficiency (Thieme, 2009). It was hypothesised that the nutrient sparing effects resulting from inclusion of DIG would significantly improve growth performance and/or feed efficiency in grower-finisher pigs fed standard diets and deliver similar performance to those of standard pigs when the grower-finisher diet digestible energy and protein specifications have been reduced by $0.4 \mathrm{MJ} \mathrm{DE} / \mathrm{kg}$.

Material and methods A 2 × 2 factorial design was used to evaluate the effects of two levels of DIG (0 and $150 \mathrm{~g} / \mathrm{t})$ and two levels of DE (CON Grower 13.9 MJ DE $/ \mathrm{kg}$ and $0.7 \mathrm{~g}$ standardised ileal digestible lysine/MJ DE (g SID lys) and Finisher 13.6 MJ DE/kg and 0.60 g SID lys/MJ DE compared with diets with DE lowered by $0.4 \mathrm{MJ} / \mathrm{kg} \mathrm{LOW}$, with the same SID:lysine ratio, effectively reducing amino acid levels as well). A total of 504 nine-week-old female pigs were allocated to 36 pens of 14 pigs per pen. All diets in the experiment were pelleted and fed ad libitum for the duration of the experiment through to sale at 21 weeks of age. Pen weights were recorded at the start of the experiment and recorded again at 3, 7, 10, and 13 weeks (sale). All deaths and removals were recorded and considered when calculating feed intake and conversion efficiency. All pigs were slaughtered at the end of the 21 week experimental period where individual hot standard carcass weight (HSCW) and fat depth at the P2 site (65 mm from the midline at the head of the last rib), and averages were calculated on a pen basis. Performance data was analysed using MANOVA (IBM SPSS Statistics for Windows, v25.0. Armonk, NY). There were no significant interactions between Specification and DIG so only the main effects are shown.

Results Feeding DIG increased $(P=0.009)$ average daily gain in the grower-finisher period, more so for pigs fed the higher energy control diets than the lower DE diets. As would be expected, feeding diets containing 0.4 MJ DE less energy reduced growth rate and P2, however addition of DIG tended to return performance to similar levels of the CON pigs not receiving the phytogenic feed additive. (Table 1).

Table 1. Effect of a phytogenic feed additive (DIG, 0 or $150 \mathrm{~g} / \mathrm{t}$ ) in a standard (CON) or a $2.5 \%$ lower energy and protein diet specification (LOW) diet on grower-finisher pig performance, across the experimental period.

\begin{tabular}{lcccccccc}
\hline & & \multicolumn{2}{c}{ CON } & \multicolumn{2}{c}{ LOW } & \multicolumn{2}{c}{$P$ value } \\
& & $0 \mathrm{~g} / \mathrm{t}$ & $150 \mathrm{~g} / \mathrm{t}$ & $0 \mathrm{~g} / \mathrm{t}$ & $150 \mathrm{~g} / \mathrm{t}$ & SEM & Specification & DIG \\
\hline BW d 0 & $(\mathrm{kg})$ & 20.7 & 20.7 & 20.7 & 20.7 & 1.1 & 0.99 & 0.99 \\
BW sale & $(\mathrm{kg})$ & 91.7 & 95.3 & 90.4 & 92.2 & 1.4 & 0.13 & 0.069 \\
ADG & $(\mathrm{kg} / \mathrm{d})$ & 0.80 & 0.84 & 0.79 & 0.80 & 0.01 & 0.026 & 0.009 \\
ADFI & $(\mathrm{kg} / \mathrm{d})$ & 1.78 & 1.86 & 1.82 & 1.82 & 0.04 & 0.94 & 0.39 \\
FCR & $(\mathrm{kg} / \mathrm{kg})$ & 2.23 & 2.21 & 2.32 & 2.27 & 0.03 & 0.039 & 0.34 \\
HSCW & $(\mathrm{kg})$ & 71.3 & 73.9 & 69.8 & 71.0 & 1.2 & 0.076 & 0.12 \\
P2 & $(\mathrm{mm})$ & 9.7 & 10.4 & 9.3 & 9.5 & 0.3 & 0.034 & 0.16 \\
\hline
\end{tabular}

ADG, average daily gain; ADFI, average daily feed intake; FCR, feed conversion ratio; BW, body weight; HSCW, hot standard carcass weight; P2, carcass backfat at the P2 site, $65 \mathrm{~mm}$ from the midline at the head of the last rib.

Conclusion The poorer ADG and FCR of the LOW pigs indicates the DE and amino acid restriction was successful in reducing performance. The pigs attempted to compensate for the low $\mathrm{DE}$ and amino acid density diet by consuming more feed which had negative effects on FCR. As the BW at sale and ADG of the CON pigs $+150 \mathrm{~g} / \mathrm{t}$ was higher than of the CON pigs without DIG and also the ADG of the LOW pigs $+150 \mathrm{~g} / \mathrm{t}$ was similar to that of the CON pigs without DIG the hypothesis was partly accepted. The inclusion of DIG in pig diets improved the growth rate in grower-finisher pigs, particularly those fed a higher energy feeding program and tended to improve feed use efficiency at both DE levels tested. Lowering DE levels by $0.4 \mathrm{MJ} / \mathrm{kg}$ can be used to reduce feed costs and manipulate growth rates and carcass P2 measurements and Digestarom ${ }^{\circledR}$ supplementation may help in returning performance to normal levels.

Acknowledgements This experiment was funded by Biomin Singapore and supported by staff at Research \& Innovation Unit at Rivalea Australia Pty Ltd.

\section{References}

Thieme R 2009. International Pig Topics. 24, 25-27. 


\section{Improving growth performance of 6 to $13 \mathrm{~kg}$ nursery pigs}

K Horgan 1 , N Browne ${ }^{1}$, J A Taylor-Pickard ${ }^{1}$, E D Frugé ${ }^{2}$, A J Gerhart ${ }^{2}$, E L Hansen ${ }^{2}$, S A Hansen ${ }^{2}$ and K A Frerichs ${ }^{2}$

${ }^{1}$ Alltech Bioscience Centre, Ireland, ${ }^{2}$ Hubbard Feeds, USA

jpickard@alltech.com

Application Dietary butyrate-based supplements have the potential to improve pig production efficiency.

Introduction Early piglet growth is dependent on developing good gut health with a stable and diverse gut microbial population. Butyrate is known to provide energy for enterocytes and is involved in gene expression, cell differentiation and gut tissue development in young mammals, and, as such, plays an important role in maintaining gut health (Biagi et al., 2007; Bedford and Gong, 2018). This butyrate-based supplement contains components known to promote gastrointestinal tract tissue growth. As growth proceeds, gut microbes produce short chain fatty acids that help tilt the microbial balance toward beneficial species. This supplement helps the young gut get ready for rapid growth and encourages feed intake.

Material and methods Two trials were conducted to determine the effects of a blend of butyric acid, Saccharomyces cerevisiae and zinc (Viligen ${ }^{\mathrm{TM}}$, Alltech Inc., Nicholasville, KY, USA) on nursery pig performance. Both studies were randomised complete block designs with six pen replicates per treatment and 28 pigs per pen. Dietary treatments consisted of a control diet (CON) or the control diet with the supplement (SUP) fed as pelleted diets without antibiotics and with 2000 ppm dietary zinc. In trial 1 and 2, 336 mixed-sex pigs (TN Tempo x TN70), $6.33 \pm 0.26 \mathrm{~kg} \mathrm{BW,} 5.92 \pm 0.35 \mathrm{~kg}$ BW, were used in each 22 and $24 \mathrm{~d}$ trial, respectively. Pen weights and feed disappearance were recorded on d 0, 11, 22 or 24 and used to calculate ADG, ADFI, and G:F. Growth data were analysed using GLM procedures (SAS Institute Inc., Cary, $\mathrm{NC}$, USA). In addition, the effect of SUP was studied in growing IPEC cell cultures, whereby gene expression for various genes related to gut transport systems (EAAT-3, PEP-T, CAT-1, y+LAT-1, ASCT, BO+AT, SGLT-1 and GLUT-2) was assessed. IPEC-J2 cells in culture were exposed to SUP for $72 \mathrm{~h}$. Cells were counted after $24 \mathrm{~h}$ growth intervals using a haemocytometer and glucose concentration of the cell medium was determined using a glucose Trinder assay (SigmaAldrich, St. Louis, MO, USA). Gene expression patterns were assessed by qPCR (Applied Biosystems 7500 Fast qPCR).

Results When the data from both trials were analysed together, pigs fed SUP had improved ADG (292 g/d vs. $259 \mathrm{~g} / \mathrm{d} ; P$ $<0.01)$, ADFI $(333 \mathrm{~g} / \mathrm{d} v s 302 \mathrm{~g} / \mathrm{d} ; P<0.01)$, and BW gain $(6.70 \mathrm{~kg}$ vs $5.94 \mathrm{~kg} ; P<0.01)$. A reduction in injectable medication use $(15.8 \%$ vs $25.6 \% ; P<0.03)$ was noted in pigs fed SUP. Mortality $(0.30 \%$ vs $1.49 \%)$ and morbidity $(4.17 \%$ vs $5.65 \%)$ did not differ between treatments $(P>0.10)$ however were consistently lower for pigs fed diets containing SUP. From the gene expression analysis, SUP had a positive impact on genes involved in amino acid transport and glucose uptake. Genes involved with Arginine uptake were upregulated in the SUP treated cells. Glucose transporter GLUT-2 was significantly $(P<0.01)$ increased in response to SUP treatment which correlated with the lower abundance of glucose available in the media of growing IPEC cells.
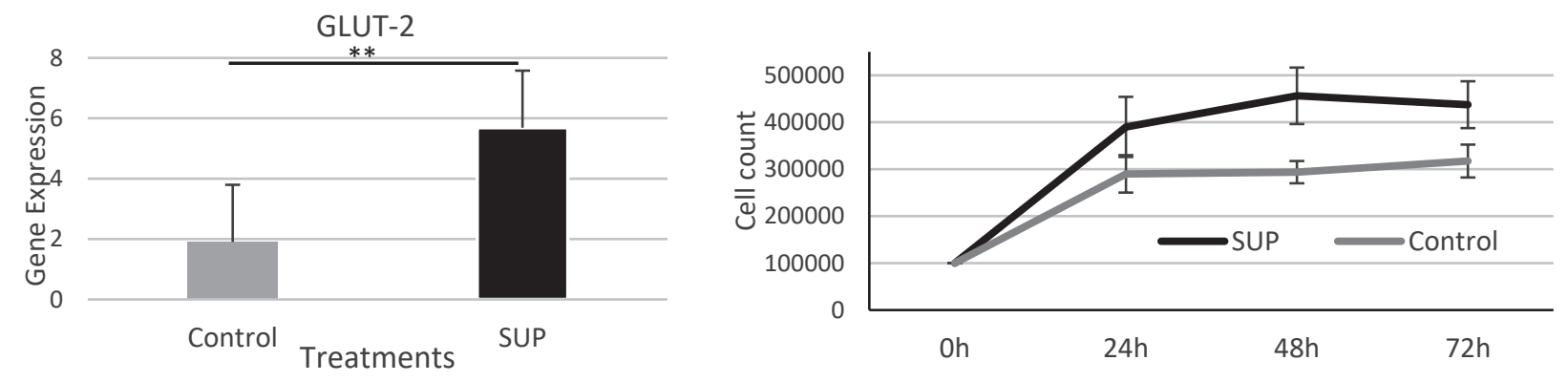

Figure 1. Gene expression of GLUT-2/ SLC5A1: Glucose transporter (left), and SUP impact on intestinal cell growth (right). Data analysed by One-way ANOVA with Tukey's multiple comparison test and significance marked as $* *(P<0.05)$

Conclusion The inclusion of SUP in young nursery pig diets improved growth performance and reduced medication requirements. The trend for reduced mortality and morbidity may result in more pigs reaching the finisher stage of production. The application of SUP upregulated gene expression, especially for the GLUT-2 glucose transporter gene. These changes are consistent with observed increases in epithelial cell proliferation and apparent glucose utilisation in cell culture systems (Zakrzewski et al., 2013).

\section{References}

Bedford A and Gong J 2018. Animal Nutrition 4, 151-159.

Biagi G, Piva A, Moschini M, Vezzali E and Roth FX 2007. Journal of Animal Science. 85, 1184-1191

Zakrzewski SS, Richter JF, Krug SM, Jebautzke B, Lee I-FM, Rieger J, Sachtleben M, Bondzio A, Schulzke JD, Fromm M and Günzel D 2013. PLoS ONE 8, e79643. 


\section{Lupin cell-wall in vitro solubilisation with combination of exogenous feed enzyme products} $\mathrm{K}$ Stamatopoulos $^{1}$, E A Della $\mathrm{Pia}^{2}$ and N R Pedersen ${ }^{2}$

${ }^{1}$ DSM Nutritional Products, Australia ${ }^{2}$ Novozymes A/S, Denmark

Kostas.Stamatopoulos@dsm.com

Application To increase the use of narrow leaf lupins as an alternative protein source in pig feed. Cell walls of lupin contain non-starch polysaccharide (NSP) that can affect nutrient digestibility and feed passage through the gut. Therefore, using exogenous feed enzymes that specifically target lupin NSP, may improve the digestibility of NSP's in monogastric animals by reducing gut viscosity, decreasing GI transient time and increasing nutrient digestibility.

Introduction Lupinus angustifolius is a species of lupin known also as narrow-leaved or blue lupin. In their raw, unprocessed form, lupins have many desirable characteristics for feeding livestock. Australia is the dominant producer of lupins worldwide producing about 800000 tonnes per year for use in the feed industry. L. angustifolius is a valuable feed ingredient for the pig industry and is used effectively in diets for most classes of pigs, constituting up to $20 \%$ of feed. Almost a third of lupin dry matter is composed of proteins not available to the animal as they are mostly encapsulated in fibres both soluble and insoluble (13\% of dry matter). Presence of soluble fibre increases viscosity and insoluble fibre increases water binding capacity, thereby increasing GI tract transit time. Pigs and poultry do not possess endogenous NSP or fibre degrading enzymes, using relevant exogenous NSP enzymes targeting lupin cell wall polysaccharides will help to decrease the antinutritional effects of cell wall NSP, as well as an increase the availability of protein, leading to improving its application as an alternative protein source in swine feed.

Material and methods Three enzyme products were investigated in this study. A multicomponent product produced by A. aculeatus containing pectinases, hemicellulases, and other cell-wall degrading carbohydrases (RONOZYME® VP (DSM Nutritional Products Ltd, Kaiseraugst, Switzerland)), a multicomponent product from T. reesei containing xylanases, $\beta$-glucanases and other fibre-degrading enzymes (RONOZYME® MultiGrain (MG)) and a monocomponent xylanase produced by an $A$. oryzae strain (RONOZYME® WX). L.angustifolius samples milled to 0.5 mm were incubated at $40^{\circ} \mathrm{C}$ for 4 hours at pH 5.0 both in the absence (control) and presence of different combination of enzymes at 2000 ppm. Following incubations, NSP analyses of soluble and insoluble fractions were performed according to Theander $e t$ al., (1995). For microscopy, lupins were embedded in paraffin, sectioned in $5 \mu \mathrm{m}$ slices and imaged by using the Confocal Microscope (Olympus, Japan). Differences between treatments were determined by ANOVA.

Results Both combinations of enzymes significantly solubilised rhamnogalacturonan, arabinan and galactan polymers as measured by the decrease in rhamnose, arabinose and galactose in the insoluble fraction and by the concomitant increase in the solubilised fractions (Table 1). A numerical decrease in the insoluble xylan content was also measured as xylose. Microscopy data clearly showed solubilisation of cell walls with enzyme as well increased visibility of the protein fraction which was only partially visible due to encapsulation by the cell walls (Figure 1).

Table 1. Enzyme effects on soluble and insoluble polysaccharides of lupin, identified as neutral sugars using NSP method incubated without enzyme or with a combination of a multicomponent cell-wall degrading enzyme (VP) with either a multicomponent fibre-degrading enzyme (VP+MG) or a monocomponent xylanase (VP+WX).

\begin{tabular}{cccccccc}
\hline Enzyme dosage & Rhamnose & Fucose & Arabinose & Xylose & Mannose & Galactose & Glucose \\
\hline Soluble NSP & & & & & & & \\
Control & $0.04^{\mathrm{b}}$ & $0.01^{\mathrm{a}}$ & $0.30^{\mathrm{b}}$ & $0.15^{\mathrm{a}}$ & $0.15^{\mathrm{a}}$ & $1.54^{\mathrm{b}}$ & $0.08^{\mathrm{a}}$ \\
VP+MG & $0.13^{\mathrm{a}}$ & $0.01^{\mathrm{a}}$ & $0.55^{\mathrm{a}}$ & $0.13^{\mathrm{a}}$ & $0.11^{\mathrm{a}}$ & $3.89^{\mathrm{a}}$ & $0.08^{\mathrm{a}}$ \\
VP+WX & $0.13^{\mathrm{a}}$ & $0.01^{\mathrm{a}}$ & $0.52^{\mathrm{a}}$ & $0.12^{\mathrm{a}}$ & $0.11^{\mathrm{a}}$ & $3.61^{\mathrm{a}}$ & $0.08^{\mathrm{a}}$ \\
Insoluble NSP & & & & & & & \\
Control & $0.64^{\mathrm{a}}$ & $0.11^{\mathrm{a}}$ & $3.66^{\mathrm{a}}$ & $2.75^{\mathrm{a}}$ & $0.75^{\mathrm{a}}$ & $15.09^{\mathrm{a}}$ & $12.92^{\mathrm{a}}$ \\
VP+MG & $0.53^{\mathrm{b}}$ & $0.09^{\mathrm{ab}}$ & $2.96^{\mathrm{b}}$ & $2.52^{\mathrm{a}}$ & $0.61^{\mathrm{b}}$ & $11.06^{\mathrm{b}}$ & $12.32^{\mathrm{a}}$ \\
VP+WX & $0.54^{\mathrm{b}}$ & $0.10^{\mathrm{a}}$ & $3.08^{\mathrm{b}}$ & $2.55^{\mathrm{a}}$ & $0.66^{\mathrm{ab}}$ & $11.49^{\mathrm{b}}$ & $12.50^{\mathrm{a}}$ \\
\hline
\end{tabular}

${ }^{\mathrm{a}, \mathrm{b}}$ Means within fibre fraction and column with different superscripts differ significantly (Tukey-Kramer $P<0.05$ )

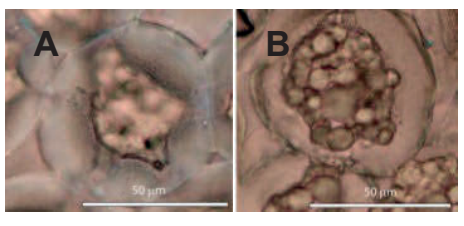

Figure 1. A: Section of lupin cell wall showing globular protein bodies (GBP)

$\mathrm{B}$ : Cell walls appearing thinner with GBP more visible after enzyme treatment.
Conclusion NSP content of lupins indicated presence of both soluble and high levels of insoluble pectin components such as rhamnogalacturonan, arabinan, galactan and xylan as deduced from the neutral NSP sugar data. This in vitro data which needs to be tested in vivo showed that the enzymes selectively solubilised the insoluble NSP, most likely increasing fermentable fibre as well as unlocked the protein, that would be more available to endogenous proteases.

References Theander O, Åman P, Westerlund E, Andersson R and Pettersson D 1995. Journal of AOAC International. 78, 1030-1044. 


\section{Evaluation of different feed acidification strategies for improvement of growth performance and economics of nursery pigs under commercial conditions}

L Lahaye $^{1}$, G Tactacan ${ }^{1}$, and R G Campbell ${ }^{2}$

${ }^{1}$ Jefo Nutrition Inc., Canada, ${ }^{2} R G$ Campbell Advisory, Australia

1lahaye@jefo.com

Application Feed acidifiers positively influence growth performance and economic profitability, but they are not all equal. Formula and presentation form matters.

Introduction Feed acidifiers have been extensively reviewed (Roth and Kirchgessner, 1998) and are commonly used in order to improve growth performance in nursery pigs. Nevertheless, acidifiers may differ in composition and supplied, either as free acidifiers or microencapsulated. This study hypothesised that nursery pig performance and economic benefits differs when different acidifier blends are presented in free or encapsulated form.

Material and methods A total of 2160 mixed-sex pigs weighing an average of $7.0 \mathrm{~kg}$ were housed in two commercial wean-to-finish production research facilities. Weaned pigs were allotted to one of four treatment groups and fed from 122 days after weaning. Basal diet contained Oxytetracycline at $220 \mathrm{ppm}$ and carbadox at $55 \mathrm{ppm}$ (13.9 MJ digestible energy (DE) $/ \mathrm{kg}, 1.40 \%$ standardized ileal digestible lysine). Treatments were Non-Acidified control (NA) with no acidifiers, Free acidification \#1 (FA1) containing a commercial blend of phosphoric, fumaric, lactic and citric acids included at $2 \mathrm{~kg} / \mathrm{t}$, Free acidification \#2 (FA2) containing a commercial blend of phosphoric, citric, malic and tartaric acids included at $1.3 \mathrm{~kg} / \mathrm{t}$ and a Microencapsulated formula of organic acids and essential oils $[\mathrm{P}(\mathrm{OA}+\mathrm{EO})]$ included at 1 $\mathrm{kg} / \mathrm{t}$ (fumaric, sorbic, malic, citric acid, thymol, vanillin and eugenol). Pen averages were used as the experimental units with 24 pens per treatment. Each pen had 22 to 23 pigs, balanced across treatments. Data were analysed by ANOVA and differences between treatments were determined by LSD with $P \leq 0.05$ considered to be statistically significant and $P \leq$ 0.10 considered as a trend (XLSTAT 2019, Addinsoft Inc, Long Island City, NY, USA).

Results The supplementation of a blend of free phosphoric, fumaric, lactic and tartaric acids (FA2) and a microencapsulated formula of organic acids and essential oils $[\mathrm{P}(\mathrm{OA}+\mathrm{EO})]$ had a significant positive impact on growth rate $(+7.9 ;+10.1 \%)$ of nursery pigs by comparison to the control treatment (Table 1$)$. Both the blend of phosphoric, citric, malic and tartaric acids (FA2) and the microencapsulated formula of organic acids and essential oils [P(OA+EO)] statistically improved feed efficiency by 7.6 and $6.9 \%$ respectively compared to the non-acidified diet and resulted to be the most profitable treatments by providing an additional margin over feed costs of $\$ 0.24$ and $\$ 0.26$ per pig respectively compared to the NA diet (Table 1).

Table 1. Weight and growth performance of control nursery pigs fed non-acidified diet (NA) compared to nursery pigs receiving $2 \mathrm{~kg} / \mathrm{t}$ of Free acidification \#1 (FA1), $1.3 \mathrm{~kg} / \mathrm{t}$ of Free acidification \#2 (FA2) or $1 \mathrm{~kg} / \mathrm{t}$ of a microencapsulated formula of organic acids and essential oils $[\mathrm{P}(\mathrm{OA}+\mathrm{EO})]$.

\begin{tabular}{ccccccc}
\hline & NA & FA1 & FA2 & P(OA+EO) & SED & $P$ value \\
\hline Day 0-22 & & & & & & \\
ADG (g/d) & $178^{\mathrm{b}}$ & $183^{\mathrm{b}}$ & $192^{\mathrm{a}}$ & $196^{\mathrm{a}}$ & 8.2 & 0.05 \\
ADFI (g/d) & 231 & 231 & 227 & 236 & 5.9 & 0.70 \\
FCR (g/g) & $1.31^{\mathrm{b}}$ & $1.30^{\mathrm{c}}$ & $1.21^{\mathrm{a}}$ & $1.22^{\mathrm{a}}$ & 0.038 & 0.001 \\
BW d 0 (kg) & 6.99 & 7.03 & 6.99 & 6.99 & 0.071 & 0.90 \\
BW d 22 (kg) & $10.07^{\mathrm{y}}$ & $10.16^{\mathrm{xy}}$ & $10.30^{\mathrm{x}}$ & $10.34^{\mathrm{x}}$ & 0.190 & 0.10 \\
Mortality (\%) & 0.7 & 1.1 & 0.7 & 0.4 & 0.37 & 0.60 \\
Additional profit per pig, USD & 0.00 & 0.02 & 0.24 & 0.26 & - & - \\
\hline
\end{tabular}

Means in a row with different superscripts differ significantly ( $\left.{ }^{\mathrm{a}, \mathrm{b}, \mathrm{c}} P \leq 0.05,{ }^{\mathrm{x}, \mathrm{y}} P \leq 0.10\right)$; ADG, average daily gain;

ADFI, average daily feed intake; FCR, feed conversion ratio; BW, body weight; SED, standard error of difference of the means. Additional profit per pig calculated as the difference between the additional gain in BW minus the additional feed costs.

Conclusion Over the 3 different acidification strategies tested, the Free Acidification \#2 and the microencapsulated formula of organic acids and essential oils [P(OA+EO)], improved nursery growth rate by $7.9 \%$ and $10.1 \%$, feed efficiency by 7.6 and $6.9 \%$ and profit per pig by $\$ 0.24$ and $\$ 0.26$ respectively when compared to non-acidified diet.

\section{References}

Roth FX and Kirchgessner M 1998. Journal of Animal Feed Science. 7 (Suppl. 1), 25-33. 


\section{Soybean meal in weaner diets improves growth performance, when compared with other soybean products}

M O Sampaio $^{1}$, R J E Hewitt ${ }^{1}$, S M Tritton ${ }^{1}$, A C Corso ${ }^{1}$, D N D’Souza ${ }^{1}$ and R J van Barneveld ${ }^{1}$

${ }^{1}$ SunPork Group, Australia

marcela.sampaio@sunporkfarms.com.au

Application There are numerous soybean products available for inclusion in weaner diets, differing in complexity of processing and price. This study shows that the use of cheaper soybean meal will not compromise performance.

Introduction Soybeans are the most popular ingredient used in animal nutrition when it comes to protein source. They contribute protein of high quality, being rich in the limiting amino acids lysine, threonine, and tryptophan that are relatively low in our energy component cereal grains (Stein et al., 2013). There are a range of soybean products available for inclusion in diets, ranging from less refined products like full-fat soybeans, which has its oil content preserved ( $20 \%)$, through to soybean meal that has the oil removed from it such that the oil content is reduced to between 1.5 to $5.0 \%$, depending on extration method. With the extraction of oil there is also a change in the crude protein content (37.5\% in full-fat soybeans vs 45 to $47 \%$ in soybean meal). Further refined products are also available such as soy protein concentrate, where soluble carbohydrates are removed, resulting in a product with approximately $65 \%$ protein (Stein et al., 2013). These products also vary in price with full-fat soybeans being 1.5 times of soybean meal, whilst soy protein concentrates are 3 times the price. However, there has been some caution in use in the newly weaned pig, as without appropriate heat-treatment anti-nutritional factors such as trypsin inhibitors and lectin may be an issue, whilst soy proteins can be allergenic (Gu et al. 2010). We hypothesised that soybean meal could be substituted for full-fat soybean and soy protein concentrate in weaner diets with no impact on performance.

Material and methods Five hundred and sixty male and female pigs ( $20 \mathrm{~d}, 5.27 \pm 0.17 \mathrm{~kg}$ ) entered the experiment over a two-week period and were sorted by sex and size and assigned to pens $(n=14)$. Pens were weighed and allocated to one of two treatments using a randomised block design, resulting in 10 replicates per treatment. Treatments consisted of isoenergetic and isonitrogenous weaner diets (14.9 MJ DE $/ \mathrm{kg}, 0.87 \mathrm{~g}$ standard ileal digestible lysine/MJ DE) with either full fat soybean meal $(8 \%)$ plus a soy protein concentrate $(2 \%$, Wilpromil, Wilmar International, Singapore) or soybean meal (10\%). Diets and water were offered ad libitum throughout the $28 \mathrm{~d}$ experimental period. Pens and feed refusal were weighed weekly. Data were analysed by ANOVA with treatment as a fixed factor, entry week as blocking factor and entry weight as a covariate. Removals were tested for significance via chi-square analysis. Significant differences between treatments were determined by LSD $\left(P<0.05\right.$, GenStat $18^{\text {th }}$ Ed, VSN International, UK).

Results There was a significant difference $(P<0.05)$ between exit weight $(\mathrm{d} 28)$ and average daily gain (ADG) when comparing treatments, with pigs fed diets containing soybean meal gaining on average an additional $0.5 \mathrm{~kg}$ at the end of the experimental period. Feed conversion (FCR) was also significantly different $(P<0.05)$ between treatments, with no difference in feed consumption over the experimental period.

Table 1 Mean weight of pigs at weaning (d 1) and the end of the experimental period (d 28), average daily feed intake (ADFI), average daily gain (ADG), feed conversion ratio (FCR) and removals across the experimental period for pigs receiving $8 \%$ full-fat soybean and $2 \%$ soy protein concentrate (FF SB + SPC) or $10 \%$ soybean meal (SBM).

\begin{tabular}{lcccccc}
\hline & $\begin{array}{c}\mathrm{d} 1 \text { weight } \\
(\mathrm{kg})\end{array}$ & $\begin{array}{c}\mathrm{d} 28 \text { weight } \\
(\mathrm{kg})\end{array}$ & $\begin{array}{c}\text { ADFI } \\
(\mathrm{kg} / \mathrm{d})\end{array}$ & $\begin{array}{c}\text { ADG } \\
(\mathrm{kg} / \mathrm{d})\end{array}$ & $\begin{array}{c}\text { FCR } \\
(\mathrm{kg} / \mathrm{d})\end{array}$ & Removals \\
\hline FF SB + SPC & 5.3 & $12.8^{\mathrm{a}}$ & 0.39 & $0.267^{\mathrm{a}}$ & $1.46^{\mathrm{a}}$ & 2 \\
SBM & 5.2 & $13.3^{\mathrm{b}}$ & 0.40 & $0.287^{\mathrm{b}}$ & $1.40^{\mathrm{b}}$ & 4 \\
SED & 0.34 & 0.23 & 0.011 & 0.008 & 0.026 & \\
$P$ value & & & & & & $\chi^{2}(1) 0.67, P 0.414$ \\
$\quad$ Treatment & 0.88 & 0.028 & 0.29 & 0.010 & 0.027 & \\
$\quad$ Block & 0.55 & $<0.001$ & 0.003 & $<0.001$ & 0.13 & \\
$\quad$ Interaction & 0.76 & 0.95 & 0.22 & 0.95 & 0.40 & \\
\hline
\end{tabular}

a,b Means within columns with different superscripts differ significantly; SED, standard error of difference of means

Conclusion These results suggest that soybean meal was utilised better by the weaned pig, resulting in improved growth performance with no impact on feed intake.

\section{References}

Gu C, Pan H, Sun Z and Qin G 2010. International Journal of Molecular Sciences. 11, 1048-1056.

Stein HH, Roth JA, Sotak KM and Rojas OJ 2013. Swine Focus \#004 


\section{Including grape marc in weaner diets reduces growth performance when fed at higher levels} M O Sampaio $^{1}$, R J E Hewitt ${ }^{1}$, S M Tritton ${ }^{1}$, A C Corso ${ }^{1}$, D N D'Souza ${ }^{1}$ and R J van Barneveld ${ }^{1}$

${ }^{1}$ SunPork Group, Australia

marcela.sampaio@sunporkfarms.com.au

Application There is a need to investigate alternatives to antibiotics to support the weaner pig during the transition from milk to meal. Secondary plant compounds, such as the phenolic compounds found in grape marc have shown promise in maintaining the gut during periods of low feed intake. However, in this study we saw impacts on performance when inclusion rates are too high.

Introduction When piglets are weaned they are subject to a range of stressors - nutritional, environmental, physiological and social. These stressors in combination with the transition from milk to meal, generally, lead to a reduction in feed intake which can lead to reductions in the barrier function of the gut, increased inflammation and predisposes the animal to post-weaning diarrhoea (Pluske et al., 2018). The traditional response has been to use antibiotics, often in combination with high levels of zinc oxide, to suppress the growth of bacteria such as Escherichia coli and Salmonella. However, both approaches have led to the development of antimicrobial resistance. The use of plant by-products to reduce feed costs is common in pig production. Whilst they are primarily used for their macronutrient content, they are often rich in secondary compounds which can enhance growth performance (Gessner et al., 2017). Grape marc is the solids remaining after grapes are pressed for juice, containing the skin, pulp seed and stems of the grape (Fontana et al., 2013). As an inexpensive source of fibre it is commonly used in cattle feed, but the presence of phenolic compounds (Cotoras et al., 2014) has seen interest from the monogastric industries. We hypothsise that the inclusion of grape marc in the diet of weaner pigs will improve growth performance.

Material and methods Five hundred and sixty male and female pigs (20 days of age, $5.79 \pm 0.15 \mathrm{~kg}, 50: 50$ male:female) entered the experiment over a four-week period, were sorted by sex and size and assigned to pens ( $\mathrm{n}=14$ ). Pigs within each pen were weighed and allocated to one of four treatments using a randomised block design, resulting in 10 replicates per treatment, with pen as the replicate. Treatments consisted of isoenergetic and isonitrogenous first stage weaner diets (14.9 MJ digestible energy (DE)/kg, $0.88 \mathrm{~g}$ standard ileal digestible lysine/MJ DE) including either 0\%, 0.133\%, 0.266\% or $0.4 \%$ of grape marc (Acti-Meal, Tarac Technologies Pty Ltd, SA). Data were analysed via ANOVA with week of entry and sex as factors, (IBM SPSS Statistics for Windows, Version 25.0. Armonk, NY: IBM Corp.), with pairwise differences between treatments determined by LSD $(P<0.05)$.

Results Pigs from the control treatment ( $0 \%$ inclusion of Grape Marc) and the treatment with inclusion of $0.133 \%$ of grape marc presented ADG significantly $(P<0.05)$ higher than the two higher inclusion rate treatments. There was no significant difference in feed conversion observed, with the growth response reflecting at least a numerical decline in feed intake as inclusion rate increased. Removals did not differ significantly between treatments.

Table 1 Mean weight of pigs at weaning (d 1) and the end of the experimental period (d 28), average daily feed intake (ADFI), average daily gain (ADG), feed conversion ratio (FCR) and removals across the experimental period for pigs receiving grape marc in the diet at $0 \%$ (Control), $0.133 \%$ (GM 0.133), 0.266\% (GM 0.266, or 0.4\% (GM 0.400).

\begin{tabular}{lcccccc}
\hline & $\begin{array}{c}\mathrm{d} 1 \text { weight } \\
(\mathrm{kg})\end{array}$ & $\begin{array}{c}\mathrm{d} 28 \text { weight } \\
(\mathrm{kg})\end{array}$ & $\begin{array}{c}\text { ADFI } \\
(\mathrm{kg} / \mathrm{d})\end{array}$ & $\begin{array}{c}\text { ADG } \\
(\mathrm{kg} / \mathrm{d})\end{array}$ & $\begin{array}{c}\text { FCR } \\
(\mathrm{kg} / \mathrm{d})\end{array}$ & Removals \\
\hline Control & 5.7 & 13.7 & 0.42 & $0.285^{\mathrm{a}}$ & 1.46 & 1 \\
GM 0.133 & 5.6 & 13.3 & 0.40 & $0.276^{\mathrm{a}}$ & 1.46 & 1 \\
GM 0.266 & 6.1 & 13.3 & 0.40 & $0.259^{\mathrm{b}}$ & 1.53 & 1 \\
GM 0.400 & 5.7 & 12.8 & 0.38 & $0.257^{\mathrm{b}}$ & 1.49 & 3 \\
SED & 0.30 & 0.48 & 0.008 & 0.008 & 0.029 & \\
$P$ value & 0.72 & 0.66 & 0.11 & 0.038 & 0.33 & $\chi^{2}(3) 2.02, P 0.568$ \\
\hline
\end{tabular}

a,b Means within columns with different superscripts differ significantly; SED, standard error of difference of means

Conclusion These results suggest that higher inclusion rates of grape marc in weaner diets have a negative impact on average daily gain. Whilst any long term impacts on gut health were not determined in this study, the initial impact on growth performance, means inclusion rates must be considered carefully.

\section{References}

Cotoras M, Vivanco H, Melo R, Aguirre M, Silva E and, Mendoza, L 2014. Molecules 19, 21154-2116.

Fontana AR, Antoniolli A and Bottini R 2013. Journal of Agricultural and Food Chemistry. 61, 8987-9003.

Gessner D, Ringseis R and Eder K 2017. Journal of Animal Physiology and Animal Nutrition.101, 605-628.

Pluske JR, Turpin DL and Kim J-C 2018. Animal Nutrition. 4, 187-196. 


\section{Effect of organic trace minerals in finisher pig diets on growth performance and loin muscle depth at slaughter}

A R Wilkinson ${ }^{1}$, D J Henman ${ }^{1}$, C J Brewster $^{1}$, A K Lealiifano ${ }^{1}$, A Kocher $^{2}$ and R M Delles ${ }^{3}$

${ }^{1}$ Rivalea Australia Pty Ltd, Australia, ${ }^{2}$ Alltech Lienert Australia Pty Ltd, Australia, ${ }^{3}$ Alltech, USA

dhenman@rivalea.com.au

Application Organic trace minerals added to finisher pig diets increase loin depth measured at slaughter and may therefore increase the overall meat yield of the carcass.

Introduction The binding of trace minerals (zinc, manganese, copper, iron and cobalt) to amino acids and peptides is beneficial, as they are easily absorbed and readily metabolized by animals, optimizing their performance (Yatoo et al., 2013). It was hypothesized that the addition of organic trace minerals into the diet of grower-finisher pigs would improve their growth performance to slaughter.

Material and methods The experiment was conducted over two replicates utilising a total of 417 female and 418 male pigs (Large White $x$ Landrace $x$ Duroc Synthetic; Primegro ${ }^{\text {TM }}$ Genetics, Corowa, NSW) housed in commercial pens (1314 pigs/pen and 15 pens per treatment). At 10 weeks of age pigs were allocated using a 2 x 2 factorial design with the main factors being: (1) Mineral type: inorganic mineral premix (Control) vs organic mineral premix containing Bioplex ${ }^{\circledR}$ $\mathrm{Cu}, \mathrm{Zn}, \mathrm{Mn}, \mathrm{Fe}, \mathrm{Co}$ and EconomasETM (Organic, Alltech Inc., Nicholasville, KY, USA) and (2) Sex: female or castrated male pigs (Improvac ${ }^{\circledR}$, Zoetis Inc., Rhodes, NSW). Pigs were fed the respective treatment diet in the grower and finisher phase (for a period of 11 weeks prior to slaughter). Pen weights and feed use was measured at d 0, 42 and 75 . All pigs were transported to a commercial abattoir at 21 weeks of age and slaughtered over two days. Carcasses were measured for hot standard carcass weight (HSCW), backfat thickness and loin depth at the P2 site. Data were analysed using ANOVA (IBM SPSS Statistics for Windows, v24.0. Armonk, NY).

Results Organic minerals increased $(P<0.05)$ loin depth but had no effect on any growth parameters measured (Table 1). There was a strong sex effect $(P<0.05)$ across growth parameters, in line with expectations within current commercial practice.

Table 1. Average pen performance of castrate male and female pigs fed a commercial inorganic mineral premix

\begin{tabular}{|c|c|c|c|c|c|c|c|c|}
\hline \multirow{2}{*}{ Variables } & \multicolumn{2}{|c|}{ Mineral type } & \multicolumn{2}{|c|}{ Sex } & \multirow{2}{*}{ SEM } & \multicolumn{3}{|c|}{$P$ value } \\
\hline & Control & Organic & Castrate & Female & & $\operatorname{Diet}(\mathrm{D})$ & $\operatorname{Sex}(\mathrm{S})$ & $\mathrm{D} \times \mathrm{S}$ \\
\hline \multicolumn{9}{|l|}{ Grower phase (d 0-42) } \\
\hline BW d $0(\mathrm{~kg})$ & 28.6 & 28.4 & 28.7 & 28.2 & 0.7 & 0.84 & 0.57 & 0.79 \\
\hline $\operatorname{ADG}(\mathrm{kg} / \mathrm{d})$ & 0.85 & 0.86 & $0.87^{\mathrm{a}}$ & $0.84^{\mathrm{b}}$ & 0.01 & 0.67 & 0.020 & 0.42 \\
\hline $\operatorname{ADFI}(\mathrm{kg} / \mathrm{d})$ & 1.78 & 1.78 & $1.74^{\mathrm{a}}$ & $1.82^{\mathrm{b}}$ & 0.02 & 0.82 & 0.028 & 0.62 \\
\hline FCR (kg:kg) & 2.09 & 2.07 & $1.99^{\mathrm{a}}$ & $2.16^{\mathrm{b}}$ & 0.01 & 0.28 & $<0.001$ & 0.74 \\
\hline \multicolumn{9}{|l|}{ Finisher phase ( $\mathrm{d} 43-75)$} \\
\hline BW d $43(\mathrm{~kg})$ & 65.3 & 65.4 & 65.8 & 64.9 & 1.0 & 0.96 & 0.54 & 0.61 \\
\hline $\operatorname{ADG}(\mathrm{kg} / \mathrm{d})$ & 1.05 & 1.06 & $1.13^{\mathrm{a}}$ & $0.983^{b}$ & 0.01 & 0.56 & $<0.001$ & 0.91 \\
\hline ADFI $(\mathrm{kg} / \mathrm{d})$ & 2.65 & 2.69 & $2.76^{\mathrm{a}}$ & $2.58^{\mathrm{b}}$ & 0.03 & 0.39 & $<0.001$ & 0.98 \\
\hline FCR (kg:kg) & 2.53 & 2.54 & $2.44^{\mathrm{a}}$ & $2.61^{\mathrm{b}}$ & 0.02 & 0.63 & $<0.001$ & 0.87 \\
\hline BW d $75(\mathrm{~kg})$ & 99.0 & 99.3 & $102.0^{\mathrm{a}}$ & $96.3^{\mathrm{b}}$ & 1.2 & 0.82 & 0.002 & 0.64 \\
\hline \multicolumn{9}{|l|}{ Carcass } \\
\hline HSCW (kg) & 77.1 & 77.9 & 78.4 & 76.1 & 1.0 & 0.77 & 0.25 & 0.56 \\
\hline $\mathrm{P} 2(\mathrm{~mm})$ & 11.4 & 11.3 & $11.8^{\mathrm{a}}$ & $11.0^{\mathrm{b}}$ & 0.2 & 0.52 & 0.01 & 0.51 \\
\hline Loin Depth (mm) & $54.1^{\mathrm{a}}$ & $55.6^{\mathrm{b}}$ & $53.8^{\mathrm{a}}$ & $56.2^{\mathrm{b}}$ & 0.5 & 0.05 & 0.001 & 0.86 \\
\hline Dressing Percentage $(\%)$ & 78.0 & 77.9 & $76.7^{\mathrm{a}}$ & $79.1^{\mathrm{b}}$ & 0.2 & 0.92 & $<0.001$ & 0.04 \\
\hline
\end{tabular}

a,b Means within a row with different superscripts differ significantly $(P<0.05)$; SEM, standard error of the mean; ADG, average daily gain; ADFI, average daily feed intake; BW, body weight; FCR, feed conversion rate; HSCW, hot standard carcass weight.

Conclusion Sex effects were in line with current commercial expectations. There were no significant increases in growth performance of pigs fed the organic mineral treatment, but the depth of their loin muscle at slaughter was increased and therefore positively impacted the overall meat yield of the carcass.

Acknowledgements Supported in part by Alltech Lienert Australia Pty. Ltd and Rivalea Australia Pty Ltd.

\section{References}

Yatoo MI, Saxena A, Deepa PM, Habeab BP, Devi S, Jatav RS and Dimri U 2013. Veterinary World. 6, 963-967. 


\section{Influence of Bacillus-based probiotic complex on growth performance, nutrient digestibility,} blood metabolites, and faecal E.coli and Lactobacillus counts in weaner pigs

S D Upadhaya ${ }^{1}, \mathrm{~J} \mathrm{Hu}^{1}$, H I Jung ${ }^{1}$ and I H Kim ${ }^{1}$

${ }^{I}$ Dankook University, Republic of Korea

inhokim@dankook.ac.kr

Application Feeding of a Bacillus based probiotic showed improvements in growth performance, total tract digestibility and microbiota balance of weaned pigs to 10 weeks of age and warrants further investigation as an alternative to using antibiotics as growth promoters in this feeding period.

Introduction Pig producers face the challenge of overcoming the increased restrictions on the use of antibiotics as growth promoters in animal production. Consequently, the use of probiotics has received considerable attention in recent years. It has been extensively documented that probiotics can reduce digestive disorders and improve productive parameters (Weichselbaum, 2009). The objective of the current experiment was to evaluate the effects of supplementation of a Bacillus-based probiotic complex in different doses on the growth performance, nutrient digestibility, blood metabolites, and faecal microbiota in weaning pigs.

Material and methods One hundred and twenty crossbred weaner pigs [(Yorkshire x Landrace) x Duroc, $28 \mathrm{~d}$ of age] with an average body weight (BW) of $7.84 \pm 1.75 \mathrm{~kg}$ were randomly allotted to 1 of 4 dietary treatments [5 pigs per pen ( 2 barrows and 3 gilts); 6 pens per treatment] based on body BW in a 6 week feeding trial. Treatments consisted of basal $\operatorname{diet}(\mathrm{CON}$, commercial diet without antibiotic, contained 16.61, 16.38, and 14.91 MJ digestible energy $/ \mathrm{kg}$ respectively and $1.67,1.50$, and $1.31 \%$ standard ileal digestible lysine respectively for phase 1,2 , and 3 ) and basal diet supplemented with graded levels $(0.1 \%, 0.2 \%$ and $0.3 \%)$ of probiotic complex (Powerzyme 100, B\&B KOREA Co.,Ltd., Gyunggi-do, Republic of Korea) consisting of Bacillus subtilis $\left(1.0 \times 10^{9} \mathrm{cfu} / \mathrm{g}\right)$ and Bacillus licheniformis $\left(1.0 \times 10^{9} \mathrm{cfu} / \mathrm{g}\right)$. Individual body weight of pigs was measured initially (d 0 ) and at $\mathrm{d} 7$ (end of Phase 1), $\mathrm{d} 14$ (end of Phase 2) and d 42 (end of Phase 3 ) of the experimental period and feed consumption was recorded on a pen basis during the experiment to determine average daily gain (ADG), feed intake (ADFI) and gain:feed (G:F) ratio. Blood sampling, for assessing blood urea nitrogen, creatinine and glucose as well as sampling for nutrient digestibility and faecal microbial counts was undertaken at the end of the experiment (d 42). Data were analysed as a completely randomized block design using GLM procedures (SAS Institute Inc., Cary, NC, USA). Differences between means were determined using Tukey multiple range test. In addition, linear and quadratic polynomial contrasts were performed to determine the effects of graded level of the probiotic complex with $P<0.05$ indicating significance.

Results The supplementation of $0.2 \%$ and $0.3 \%$ probiotic complex improved $(P<0.05)$ feed efficiency across the experimental period compared with CON. A linear increase $(P<0.05)$ in ADG and G:F ratio was observed in Phase 3 and across the experiment, as was a linear increase $(P<0.05)$ in BW during Phase 3 . The apparent total tract digestibility (ATTD) of dry matter, nitrogen, and energy increased linearly $(P<0.05)$. A linear increase $(P<0.05)$ in faecal Lactobacillus counts and reduction $(P<0.05)$ in faecal E.coli counts were observed but no effects on blood metabolites were observed.

Table 1. Effect of increasing levels (CON, $0.1 \%, 0.2 \%$ and $0.3 \%$ ) of a dietary Bacillus-based probiotic complex supplement on the performance of weaner pigs.

\begin{tabular}{lccccccc}
\hline & & & & \multicolumn{2}{c}{$P$ value } \\
& CON & $0.1 \%$ & $0.2 \%$ & $0.3 \%$ & SEM & Linear & Quadratic \\
\hline $\mathrm{d}$ 42 weight $(\mathrm{kg})$ & $25.35^{\mathrm{b}}$ & $26.07^{\mathrm{ab}}$ & $26.36^{\mathrm{a}}$ & $26.84^{\mathrm{a}}$ & 0.393 & 0.014 & 0.760 \\
ADG (g/d) & $417^{\mathrm{b}}$ & $434^{\mathrm{ab}}$ & $441^{\mathrm{a}}$ & $453^{\mathrm{a}}$ & 9.20 & 0.014 & 0.749 \\
ADFI (g/d) & 638 & 646 & 641 & 656 & 8.98 & 0.228 & 0.696 \\
G:F (g/g) & $0.653^{\mathrm{b}}$ & $0.672^{\mathrm{ab}}$ & $0.688^{\mathrm{a}}$ & $0.689^{\mathrm{a}}$ & 0.010 & 0.003 & 0.299 \\
ATTD Dry Matter (\%) & $81.02^{\mathrm{b}}$ & $82.14^{\mathrm{ab}}$ & $82.76^{\mathrm{a}}$ & $82.47^{\mathrm{ab}}$ & 0.402 & 0.012 & 0.096 \\
ATTD Nitrogen (\%) & $81.45^{\mathrm{b}}$ & $82.42^{\mathrm{ab}}$ & $83.61^{\mathrm{a}}$ & $83.4^{3 \mathrm{a}}$ & 0.417 & 0.001 & 0.181 \\
ATTD Energy (\%) & $80.37^{\mathrm{b}}$ & $80.82^{\mathrm{b}}$ & $82.56^{\mathrm{a}}$ & $83.44^{\mathrm{a}}$ & 0.412 & $<0.001$ & 0.609 \\
Lactobacillus $(\log 10$ cfu/g) & 7.1 & 7.09 & 7.13 & 7.22 & 0.020 & 0.020 & 0.160 \\
E.coli $(\log 10$ cfu/g) & $5.83^{\mathrm{a}}$ & $5.78^{\mathrm{a}}$ & $5.75^{\mathrm{ab}}$ & $5.67^{\mathrm{b}}$ & 0.031 & $<0.001$ & 0.692 \\
\hline
\end{tabular}

${ }^{\mathrm{a}, \mathrm{b}}$ Means in the same row with different superscript differ significantly $(P<0.05)$; SEM, standard error of means

Conclusion Supplementation of a graded level of probiotic complex showed significant linear effects in overall ADG and G:F. In addition, a dose response to probiotic supplementation was observed in nutrient digestibility and faecal E.coli counts and these findings warrant further investigation into the use of a probiotic complex as a method for reducing use of antibiotic growth promoters.

\section{References}

Weichselbaum E 2009. Nutrition Bulletin. 34, 340-373. 


\section{Increased glutamate intake did not result in higher levels in milk when supplemented to gestating and lactating sow diets}

V De Luca ${ }^{1}$, E Roura $^{2}$ and S A Guzmán-Pino ${ }^{1}$

${ }^{1}$ Universidad de Chile, Chile, ${ }^{2}$ The University of Queensland, Australia

sguzmanp@uchile.cl

Application Dietary inclusion of taste active compounds such as monosodium glutamate (MSG) in sows did not influence milk composition and/or growth performance of postweaning pigs.

Introduction Weaning represents a major stress problem for piglets affecting their welfare and the profitability of pig farms. In order to improve feed intake of animals at this critical phase, maternal flavour transference and continuity has been studied in recent years (Figueroa et al., 2013). However, most of the work in pigs has been around feed volatiles while little attention has been focused on taste active compounds such as MSG, a compound known to elicit umami taste in humans and pigs (Roura et al., 2011). The objective of this study was to analyse the changes in milk amino acid composition and postweaning piglet performance after MSG supplementation into gestating and lactating diets. The hypothesis was that dietary MSG supplementation in sows increases the glutamate concentration in milk and improves growth in postweaning piglets.

Material and methods Sixteen sows (Landrace x Large White) and 156 piglets from their litters, were assigned to two experimental groups (control or MSG) $(\mathrm{n}=8)$. Sows were selected based on similar parity number $(2.6 \pm 0.8(\mathrm{mean} \pm \mathrm{SD}))$, body condition score $(3.5 \pm 0.6)$ and P2 backfat thickness $(9.6 \pm 2.2 \mathrm{~mm})$. The two groups were offered standard commercial gestating and lactating programs in mash feeds. MSG treatment consisted of the addition of $50 \mathrm{~g}$ of MSG, per $\mathrm{kg}$ of feed. During gestation, $2.4 \mathrm{~kg} / \mathrm{d}$ of feed was given to sows on a restricted diet, while sows in lactation were given $6.0 \mathrm{~kg} / \mathrm{d}$. Following an intramuscular injection of $20 \mathrm{IU}$ of oxytocin, 6 milk samples were collected on d 7 after farrowing from three sows of each experimental group. Samples were analysed for amino acid composition by HPLC. Pigs born from these sows were allocated into 6 nursery pens (26 pigs/pen) after weaning, with 3 pens for each experimental group. Animals were fed standard commercial pre-starter and starter diets until $21 \mathrm{~d}$ postweaning. In pigs born from MSG-fed sows, diets were supplemented with $0.1 \mathrm{mM}$ of MSG (based on previous studies). Body weight of pigs was individually recorded on d 0, 3, 7, 14 and 21 after weaning. Data were analysed with ANOVA by using the GLM and MIXED procedures of SAS (SAS Institute Inc., Cary, NC, USA).

Results MSG supplementation did not significantly $(P>0.05)$ impact the glutamic acid (or any other essential amino acid) content in milk (Table 1). No differences $(P>0.05)$ due to the ordinal number of parities were also observed in this experiment. No significant differences $(P>0.05)$ in postweaning piglet performance between the two groups were observed in this trial (data not shown).

Table 1. Comparison of the essential amino acid and glutamic acid sow milk composition (mg/100 ml) of a control compared to a dietary glutamate supplemented (MSG) group.

\begin{tabular}{lccccc}
\hline \multirow{2}{*}{$\begin{array}{l}\text { Free amino acids in milk } \\
(\mathrm{mg} / 100 \mathrm{ml})\end{array}$} & \multicolumn{2}{c}{ Treatment } & \multicolumn{2}{c}{$P$ value } \\
\hline Glutamic acid & 1272.5 & 1181.2 & 27.9 & 0.14 & 0.31 \\
Histidine & 129.2 & 119.8 & 6.7 & 0.42 & 0.51 \\
Arginine & 206.9 & 201.0 & 7.5 & 0.63 & 0.22 \\
Threonine & 197.3 & 200.7 & 8.9 & 0.81 & 0.91 \\
Tyrosine & 69.2 & 78.3 & 9.2 & 0.56 & 0.53 \\
Valine & 282.0 & 268.1 & 11.8 & 0.49 & 0.90 \\
Methionine & 53.1 & 63.7 & 2.6 & 0.10 & 0.17 \\
Isoleucine & 246.6 & 229.8 & 10.6 & 0.38 & 0.77 \\
Leucine & 449.0 & 435.0 & 20.4 & 0.67 & 0.97 \\
Phenylalanine & 207.4 & 202.0 & 13.1 & 0.79 & 0.94 \\
Lysine & 388.3 & 383.6 & 31.2 & 0.92 & 0.80 \\
\hline
\end{tabular}

Conclusion It is concluded that MSG supplementation in gestating and lactating sows did not affect milk amino acid profiles or postweaning piglet performance.

\section{References}

Figueroa J, Solà-Oriol D, Vinokurovas L, Manteca X and Pérez JF 2013. Animal Feed Science and Technology 183, 160167.

Roura E, Humphrey B, Klasing KC and Swart M 2011. Flavour and Fragrance Journal. 26, 282-285. 


\section{Glutamate inclusion in gestating and lactating diets in sows increased sweet taste sensitivity and appetite in postweaning pigs}

V De Luca ${ }^{1}$, E Roura $^{2}$ and S A Guzmán-Pino ${ }^{1}$

${ }^{1}$ Universidad de Chile, Chile, ${ }^{2}$ The University of Queensland, Australia

sguzmanp@uchile.cl

Application Monosodium glutamate (MSG) supplementation in sows may condition piglet feeding behaviour with the aim of improving postweaning feed intake.

Introduction Maternal experiences during gestation and lactation may condition the development of progeny behaviours such as those related to feeding. In particular, the continuity of selected flavours through feeds and feed ingredients has been shown to reduce stress in piglets (Oostindjer et al., 2009). The aim of this study was to evaluate the effect of MSG inclusion on the preference thresholds and sensory-motivated intake of sucrose in pigs. The hypothesis was that umami inclusion into gestation and lactation diets would condition litter feeding behaviour to increase sweet appetite following a nutrient-balancing compensatory mechanism.

Material and methods Sixteen sows (Landrace x Large White) and 208 piglets from their litters were assigned to two experimental groups offered standard commercial gestation and lactation feeding programs without (control) or with (MSG) the inclusion of $50 \mathrm{~g} / \mathrm{kg}$ of MSG. Animals born from these sows were distributed into 4 pens of 26 animals of the same age, for each experimental group at weaning. On days 5 and 6 after weaning, piglets were trained to perform preference tests using two identical drinkers with $500 \mathrm{ml}$ of water or a $200 \mathrm{mM}$ sucrose solution. Preference thresholds (sensitivity) for sucrose were determined in piglets from both groups of sows using six concentrations: $0.03,0.1,1,6,12$ and $18 \mathrm{mM}$. The threshold was identified as the lowest concentration of sucrose resulting in a preference value significantly $(P<0.05)$ higher than 50\%. Preference values were calculated as the percentage of intake of the treatment solution relative to the total fluid intake from the two drinkers (treatment and control). The statistical analysis was based on the comparison with the neutral value of 50\% using the Student's t-test. Regarding the sensory-motivated intake, the intake of the sucrose solution was compared to the intake of the negative control (water) during the choice scenario. This data was analysed with ANOVA by using the GLM procedure of SAS (SAS Institute Inc., Cary, NC, USA).

Results The sucrose threshold for the control (non-MSG) group was observed at $12 \mathrm{mM}(P=0.003)$. In contrast, the sucrose preference threshold for piglets from MSG-fed sows was identified at $1 \mathrm{mM}(P=0.032)$. Consistent with the preference results, the sensory motivated intake of the $1 \mathrm{mM}$ sucrose solution in pigs from control sows was not significant $(P=0.119)$. In pigs from MSG-fed sows, concentrations of $1 \mathrm{mM}$ and $6 \mathrm{mM}$ generated sucrose consumptions significantly greater than the negative control $(P=0.020$ and $P=0.001)$.

Table 1. Preference values and sensory-motivated intake of sucrose concentrations in postweaning pigs from control or MSG-fed sows.

\begin{tabular}{|c|c|c|c|c|c|c|c|c|}
\hline \multirow[t]{2}{*}{ Sucrose concentration (mM) } & \multicolumn{4}{|c|}{ Control } & \multicolumn{4}{|c|}{ MSG } \\
\hline & \multicolumn{2}{|c|}{ Preference $(\%)$} & SEM & $P$ value & \multicolumn{2}{|c|}{ Preference $(\%)$} & SEM & $P$ value \\
\hline 0.03 & \multicolumn{2}{|c|}{-} & - & - & \multicolumn{2}{|c|}{47.1} & 5.16 & 0.581 \\
\hline 0.1 & \multicolumn{2}{|c|}{-} & - & - & \multicolumn{2}{|c|}{62.2} & 7.51 & 0.129 \\
\hline 1 & \multicolumn{2}{|c|}{58.7} & 4.84 & 0.096 & \multicolumn{2}{|c|}{66.5} & 6.78 & 0.032 \\
\hline 6 & \multicolumn{2}{|c|}{62.7} & 6.68 & 0.081 & \multicolumn{2}{|c|}{66.7} & 6.21 & 0.019 \\
\hline 12 & \multicolumn{2}{|c|}{72.5} & 6.02 & 0.003 & \multicolumn{2}{|c|}{61.9} & 7.34 & 0.131 \\
\hline 18 & \multicolumn{2}{|c|}{62.2} & 6.95 & 0.049 & \multicolumn{2}{|c|}{66.7} & 4.42 & 0.003 \\
\hline & \multicolumn{2}{|c|}{ Intake $(\mathrm{g})$} & \multicolumn{6}{|c|}{ Intake $(\mathrm{g})$} \\
\hline & Water & Test & SEM & $P$ value & Water & Test & SEM & $P$ value \\
\hline 0.03 & - & & - & - & 65.1 & 56.2 & 12.21 & 0.611 \\
\hline 0.1 & - & & - & - & 53.3 & 74.6 & 16.11 & 0.360 \\
\hline 1 & 47.7 & 75.4 & 12.07 & 0.119 & 49.8 & 101.4 & 14.63 & 0.020 \\
\hline 6 & 92.2 & 145.5 & 22.69 & 0.110 & 64.3 & 125.7 & 15.10 & 0.001 \\
\hline 12 & 72.9 & 182.6 & 17.84 & $<0.001$ & 71.4 & 150.8 & 23.42 & 0.023 \\
\hline 18 & 125.1 & 214.5 & 25.69 & 0.021 & 115.8 & 242.5 & 21.90 & $<0.001$ \\
\hline
\end{tabular}

Conclusion Including glutamate to sow diets increased sucrose preference and sensory-motivated intake of their litters. Specific taste (nutrient) stimulation through the maternal transfer may influence the preference for other taste (nutrient) types. Understanding the influence of maternal diets on piglet preferences is critical to improve the formulation of postweaning diets.

\section{References}

Oostindjer M, Bolhuis JE, van den Brand H and Kemp B 2009. Chemical Senses. 34, 775-787. 


\section{Defining temperature groupings around mating of sows for farrowing rate using cluster analysis}

A M G Bunz $^{12}$, K L Bunter ${ }^{2}$, R S Morrison ${ }^{1}$, B G Luxford ${ }^{1}$ and S Hermesch ${ }^{2}$

${ }^{1}$ Rivalea Australia Pty Ltd, Australia, ${ }^{2}$ Animal Genetics and Breeding Unit, a joint venture of NSW Department of

Primary Industries and University of New England, University of New England, Australia

abunz@rivalea.com.au

Application The methodology of defining temperature groupings can be applied to other traits to better quantify ambient temperature effects on pig reproduction traits.

Introduction Seasons are defined by grouping calendar months according to specific climate characteristics. However, this definition of season does not account for variation within a calendar based season and between the same seasons in different years. Therefore, a more flexible approach is required to define seasons. The aim of this study was to define meaningful biological temperature based groupings relevant to mating dates of sows using cluster analysis.

Material and Methods Information about the farrowing rate (FR: 0=fail, 1=pregnancy) resulting from 55767 firstinsemination records of 17484 sows, collected from 2012 to 2017, from a single farm in southern New South Wales, Australia were available. Maximum ambient temperature of the day (Tmax) for the piggery were obtained from the nearby Rutherglen weather station $(\sim 16 \mathrm{~km})$. The climate is characteriszed by very hot summers, cool winters and low humidity. Sows were housed in naturally ventilated sheds and had drip cooling provided during their lactation period when shed temperature exceeded $30^{\circ} \mathrm{C}$. A generalized linear model with a logit link was used to identify the most informative days for FR at first insemination regarding Tmax in the time period 35 days prior to and 35 days post mating date. Additionally, the model for FR also included parity at mating, line (Large White, Landrace and Duroc, Primegro ${ }^{\mathrm{TM}}$ Genetics, Corowa NSW) and year of mating. The most informative days based on significance $(P<0.05)$ for FR were retained in the final analysis. Then, Tmax of the most informative days of every mating date was extracted for further cluster analysis. The Tmax pattern around mating was assigned according to their dissimilarity into five temperature classes using the Partitioning Around the Medoid (PAM) method in the R package cluster (Maechler et al., 2018; R Core Team, 2018). PAM cluster approach has been described by Kaufman and Rousseeuw (1990).

Results The days 34, 29 and $18 \mathrm{~d}$ before mating, as well as 3, 26, 27 and $29 \mathrm{~d}$ after mating, were only significant $(P<$ 0.05 ) and therefore most informative for FR. Average lactation length was $27 \mathrm{~d}$. Therefore, all significant days prior to mating fell into the time period a sow generally was in the farrowing house. Five clusters for temperature pattern were identified for Tmax information of informative days around the mating date. These five clusters represented trait specific temperature groupings (T-group, Table 1).

Table 1. Mean maximum temperatures $\left({ }^{\circ} \mathrm{C}\right)$ by temperature groupings (T-group) for days prior to (negative days) and post (positive days) first mating event

\begin{tabular}{cccccccccc}
\hline T-group & N. mating & \multicolumn{6}{c}{ Difference to first mating date $(\mathrm{d} 0)$ in days } & Note $^{1}$ \\
& days & -34 & -29 & -18 & +3 & +26 & +27 & +29 & \\
\hline 1 & 506 & 29.1 & 30.0 & 30.6 & 32.2 & 32.7 & 32.8 & 32.2 & high prior to and post mating \\
2 & 398 & 18.0 & 18.6 & 20.6 & 23.7 & 27.6 & 27.6 & 28.1 & low prior to and medium post mating \\
3 & 328 & 32.5 & 31.8 & 30.8 & 27.4 & 22.8 & 22.6 & 22.6 & high prior to and medium post mating \\
4 & 300 & 24.6 & 23.8 & 21.6 & 18.2 & 15.2 & 15.1 & 14.9 & medium prior to and low post mating \\
5 & 656 & 14.7 & 14.4 & 14.2 & 14.4 & 15.6 & 15.7 & 15.9 & low prior to and post mating \\
\hline \multicolumn{7}{c}{}
\end{tabular}

Conclusion Cluster analysis was able to create T-groups, which accounted for different maximum temperature histories sows were exposed to around mating event. Differences in farrowing rate at first insemination event can be explored according to these T-groups. Alternatively, other temperature variables can be evaluated to define T-groups.

Acknowledgements Supported in part by Australian Pork Limited (Project number 2017/2208 and Student scholarship 2018/007. Joel Lisonbee (Bureau of Meteorology) is thanked for his assistance.

\section{References}

Maechler M, Rousseeuw P, Struyf A, Hubert M and Hornik K. 2018. cluster: R package version 2.0.7-1.

Kaufman L and Rousseeuw PJ. 1990. Finding groups in data: an introduction to cluster analysis. pp. 68-107. John Wiley \& Sons, Hoboken, NJ, USA.

R Core Team 2018. R: A language and environment for statistical computing. R Foundation for Statistical Computing, Vienna, Austria. 


\section{Relationship between serum anti-Müllerian hormone levels and ovarian reserve in gilts}

A N Steel ${ }^{1}, \mathrm{R} Z$ Athorn ${ }^{2}$ and $\underline{\mathrm{C} \mathrm{G} \mathrm{Grupen}}{ }^{1}$

${ }^{1}$ The University of Sydney, Australia, ${ }^{2}$ Australian Pork Limited, Australia

christopher.grupen@sydney.edu.au

Application The results of this study suggest that serum anti-Müllerian hormone (AMH) levels are a useful indicator of reproductive potential in immature gilts. The ability to select gilts that have a superior ovarian reserve could improve the efficiency of pig breeding programs by increasing sow retention rates.

Introduction In species other than the pig, AMH has been found to be a good endocrine marker of both ovarian reserve and antral follicle counts (Rico et al., 2009). AMH is a member of the TGF- $\beta$ family of glycoproteins and is a key regulator of follicular growth, being produced by the granulosa cells of ovarian follicles. In pigs, AMH expression levels remain unchanged through to the large antral stage (Almeida et al., 2018), which contrasts with the AMH expression patterns observed in other species. Development from $60 \mathrm{~d}$ of age, and our previous findings indicate that measurement of serum AMH levels at $80 \mathrm{~d}$ may be predictive of large antral stage future reproductive performance (Steel et al., 2017). Hence it was hypothesised that concentrations of circulating AMH in gilts at $80 \mathrm{~d}$ of age are associated with ovarian properties at $160 \mathrm{~d}$ of age.

Material and methods Blood samples were collected from Landrace $\mathrm{x}$ Large White gilts at a commercial piggery located in Southern NSW (Primegro ${ }^{\mathrm{TM}}$ Genetics, Corowa, NSW; $\mathrm{n}=54$ ) at 80 and $160 \mathrm{~d}$ of age. Serum AMH levels were measured using an ELISA kit as previously described (Steel et al., 2018). At $160 \mathrm{~d}$ of age, the gilts were slaughtered. Carcass weight $(\mathrm{CW})$ and $\mathrm{P} 2$ fat scores were recorded and uteri and ovaries were collected. Ovaries were prepared for histological analysis and total numbers of follicles (primary, secondary, preantral and antral) were determined as described by Warren et al. (2015). Statistical analyses were conducted using the software package R (ver. 3.3.3, R Core Team 2017). Principal component (PC) analysis revealed a PC that accounted for 55.8\% of ovarian variation, referred to as Ovarian Follicle Index of total follicle count (OFI_tot). Interactions between serum AMH levels and OFI tot were assessed using multidimensional spline models.

Results The mean serum concentrations of AMH at d 80 and d 160 did not differ $(10.9 \pm 1.3 \mathrm{ng} / \mathrm{mL}$ and $11.8 \pm 1.2 \mathrm{ng} / \mathrm{mL}$, respectively; $P>0.05)$. The total numbers of follicles in ovaries at d160 ranged from 42880 to 275814 . There was a three-way interaction effect of d $80 \mathrm{AMH}, \mathrm{d} 160 \mathrm{AMH}$ and pubertal status on OFI_tot $\left(P=0.045\right.$, Adj- $\left.\mathrm{R}^{2}=0.12\right)$. In gilts that had no corpora lutea (CL) present at d 160 (immature gilts), there was an interaction between d 80 and d 160 AMH levels and OFI_tot (Figure 1), and in gilts that had CL present at d 160, there was not. Measurements of serum AMH at d 80 and d 160 were not associated with uterine properties, $\mathrm{CW}, \mathrm{P} 2$ fat scores or the presence of $\mathrm{CL}$ at $\mathrm{d} 160(P>0.05)$.

Conclusion The results show that in immature gilts there was a relationship between serum AMH concentrations and total follicle numbers such that the lower the AMH levels at both d 80 and d 160, the greater the total follicle count. Therefore, serum AMH levels measured at 80 and $160 \mathrm{~d}$ of age may be predictive of ovarian reserve. A more detailed longitudinal study is needed to confirm this relationship, and investigations into whether this could be applied on-farm to aid in the gilt selection process are warranted.

Acknowledgements Supported in part by Australian Pork Limited (Project number 2014/217).

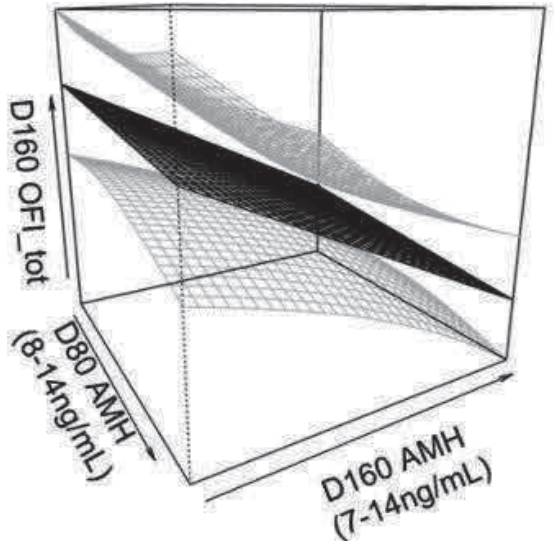

Figure 1 Three-dimensional smoothing spline model showing the two-way interaction between $\mathrm{d} 80$ and $\mathrm{d} 160 \mathrm{AMH}$ levels on OFI_tot in immature gilts (standard errors are shown in grey). Greater OFI_tot values correspond to gilts with greater overall follicle counts.

\section{References}

Almeida FRCL, Costermans NGJ, Soede NM, Bunschoten A, Keijer J, Kemp B and Teerds KJ 2018. PloS One. 13, e0197894

R Core Team 2017. R: A language and environment for statistical computing. R Foundation for Statistical Computing, Vienna, Austria.

Rico C, Fabre S, Medigue C, di Clemente N, Clement F, Bontoux M, Touze JL, Dupont M, Briant E, Benoit R, Beckers JF and Monniaux D 2009. Biology of Reproduction. 80, 50-59.

Steel AN, Athorn RZ and Grupen CG 2017. Animal Production Science. 57, 2476.

Steel A, Athorn RZ and Grupen CG 2018. Animal Reproduction Science. 195, 197-206.

Warren L, Murawski M, Wilk K, Zieba DA and Bartlewski PM 2015. Experimental Biology and Medicine. 240, 576584. 


\section{Fence-line boar exposure at the end of lactation did not improve the reproductive performance of group housed sows}

E M Ford $^{1}$, E C Greenwood ${ }^{2}$, R Z Athorn ${ }^{1}$, R S Morrison ${ }^{1}$ and W H E J van Wettere ${ }^{2}$

${ }^{1}$ Rivalea Australia Pty Ltd, Australia, ${ }^{2}$ The University of Adelaide, Australia

eford@rivalea.com.au

Application Fence-line boar exposure in conjunction with group housing at the end of lactation may not be a viable option for the pig industry to improve the reproductive performance of sows.

Introduction It has previously been demonstrated that daily fence-line boar exposure in conjunction with group housing at the end of lactation can improve reproductive performance (increased litter size) of sows mated after weaning (Greenwood, 2016). The aim of this study was to determine whether four days of fence-line boar exposure prior to weaning would reduce the wean to service interval, and increase the subsequent litter size, without stimulating the occurrence of lactational oestrus.

Material and methods In this study, 501 sows (Large White $x$ Landrace, Primegro ${ }^{\text {TM }}$ Genetics, Corowa, NSW) farrowed between June and November 2017 and were allocated to three treatment groups. The sows (parity $1-7$ ) were housed in farrowing crates and received either no boar exposure during lactation (Control); or fence-line boar exposure (Crate $+\mathrm{BE})$ for the last 4 days pre-weaning; or housed in farrowing crates until 7 days before weaning, at which point they were moved to group lactation housing and received fence-line BE for the final 4 days prior to weaning (Group $+\mathrm{BE})$. All sows were weaned on day $27 \pm 0.1$ (mean \pm SE) of lactation and mated at their first detected oestrus, except for sows that displayed oestrus in lactation, which were mated in lactation. Sows were divided into groups based on whether or not they expressed oestrus during lactation, and the effect of lactation oestrus and its interaction with treatment on all parameters was determined in a $3 \times 2$ factorial comparison using either an ANOVA (wean to service interval and total born) or a generalised linear mixed model (binomial; piglets born alive or dead; GenStat 15th ed, VSN International, Hemel Hempstead, UK). Farrowing rate was analysed using Pearson's chi square.

Results There was no treatment effect on subsequent farrowing rate or litter size. However, sows that were mated in lactation as a pooled cohort had a decreased farrowing rate $(P<0.05)$ and fewer total and live born piglets in the subsequent lactation $(P<0.1)$. The frequency of lactational oestrus was greater $(P<0.05)$ in the Group $+\mathrm{BE}(22.4 \%)$ treatment, in comparison with the Crate+BE $(8.3 \%)$ and Control $(1.5 \%)$ treatments.

Table 1. Effect of treatment (no boar exposure, Control; fence-line boar exposure during last 4 days pre-weaning in crate, Crate $+\mathrm{BE}$; fence-line boar exposure during last 4 days pre-weaning in group lactation, Group $+\mathrm{BE}$ ) and timing of mating (Lactation or Post-Wean) on subsequent reproductive performance

\begin{tabular}{lcccccc}
\hline & \multicolumn{2}{c}{ Control } & \multicolumn{2}{c}{ Crate+BE } & \multicolumn{2}{c}{ Group+BE } \\
& Lactation & Post-Wean & Lactation & Post-Wean & Lactation & Post-Wean \\
\hline No. of sows & $2^{*}$ & 129 & 13 & 143 & 28 & 97 \\
WSI, days & $0.0^{\mathrm{ac}} \pm 0.0$ & $5.7^{\mathrm{bc}} \pm 0.4$ & $-2.2^{\mathrm{ac}} \pm 1.2$ & $5.7^{\mathrm{bc}} \pm 0.4$ & $-1.8^{\mathrm{ad}} \pm 0.8$ & $5.5^{\mathrm{bd}} \pm 0.5$ \\
Farrowing rate, $\%$ & $100.0 \%$ & $86.9 \%$ & $53.0^{\circ} \%$ & $88.4 \%$ & $67.9 \%$ & $91.1 \%$ \\
Subsequent litter size & & & & & & \\
$\quad$ Total born & $13.9^{\mathrm{a}} \pm 2.5$ & $14.6^{\mathrm{b}} \pm 0.3$ & $11.9^{\mathrm{a}} \pm 1.2$ & $14.0^{\mathrm{b}} \pm 0.3$ & $12.7^{\mathrm{a}} \pm 0.8$ & $13.8^{\mathrm{b}} \pm 0.4$ \\
$\quad$ Born alive & $12.7 \pm 2.2$ & $13.0 \pm 0.3$ & $10.6 \pm 1.1$ & $12.5 \pm 0.3$ & $11.5 \pm 0.7$ & $12.5 \pm 0.3$ \\
$\quad$ Born dead & $1.1 \pm 1.1$ & $1.1 \pm 0.2$ & $1.1 \pm 0.6$ & $1.2 \pm 0.1$ & $0.5 \pm 0.4$ & $1.0 \pm 0.2$ \\
\hline
\end{tabular}

${ }_{\mathrm{a}, \mathrm{b}}$ Means within a row and treatment with different superscripts differ significantly $(P<0.05)$; ${ }^{\mathrm{c}, \mathrm{d}}$ Means within a row with different superscripts differ significantly $(P<0.01)$; WSI, wean to service interval; *by design, the control treatment was not expected to show lactation oestrus, data is included for transparency.

Conclusion Providing sows with daily, fence-line boar exposure for four days prior to weaning did not improve the reproductive performance of sows mated following weaning. Fence-line boar exposure in conjunction with group housing increased the incidence of lactation oestrus. Sows mated during lactation experienced a decrease in fertility and fecundity compared to those that were mated post-weaning.

Acknowledgements Supported in part by Pork CRC Limited, staff at the Research and Innovation Unit and farming operations at Rivalea Australia Pty Ltd are thanked for their support.

\section{References}

Greenwood EC 2016. Ph.D Thesis. The University of Adelaide. 


\title{
Piglet birth weight does not correlate with umbilical venous-arterial glucose difference measured at birth
}

\author{
$\underline{\text { F Liu }}^{1}$, B J Leury ${ }^{2}$, E M Ford ${ }^{1}$, R J Smits ${ }^{1}$, R S Morrison ${ }^{1}$, C J Brewster ${ }^{1}$, D J Henman ${ }^{1}$, W Zhao ${ }^{2}$, J J Cottrell ${ }^{2}$, F R \\ Dunshea $^{2}$ and A W Bell ${ }^{3}$ \\ ${ }^{1}$ Rivalea Australia Pty Ltd, Australia, ${ }^{2}$ The University of Melbourne, Australia, ${ }^{3}$ Cornell University, USA \\ fliu@rivalea.com.au
}

Application Piglet birth weight does not correlate with umbilical venous-arterial glucose difference measured at birth, implying that umbilical blood flow may be a key determinant of foetal pig development. Developing technologies that can increase umbilical blood flow may be able to increase piglet birth weight.

Introduction Glucose is the major carbohydrate energy source for foetal development during gestation (e.g. Fowden et al., 1997). Net foetal uptake of glucose, or the amount of glucose utilised by the foetal tissues, is ultimately determined by maternal blood glucose concentration, placental transfer, the umbilical venous-arterial concentration difference and umbilical blood flow (Hay Jr., 1991). Heavier foetuses presumably require increased uptake of glucose which may be reflected in a larger difference in venous-arterial glucose concentration. Thus, we hypothesised that the umbilical venousarterial blood glucose concentration difference at birth is positively correlated with piglet birth weight.

Material and methods A total of 135 newborn piglets that were born to 50 mixed parity sows (Large White $\mathrm{x}$ Landrace, Primegro $^{\mathrm{TM}}$ Genetics, Corowa, NSW) were randomly selected and assessed in the farrowing house of Rivalea, Australia. A pair of haemostatic clamps were used to clamp a 5-10 cm length of umbilical cord when the piglet (born alive) was expelled from the reproductive tract while the umbilical cord was still intact. Residual blood was immediately collected from the umbilical vein and artery of the clamped umbilical cord via syringe and analysed in a glucose meter (Accu-Chek Performa, Roche, Castle Hill, NSW; Sanchez et al., 2016), generally within 2 minutes. Piglets were weighed individually using a digital scale prior to sucking. Within 10 minutes of glucose measurement in umbilical cords, a blood sample from the farrowing sow was collected by pricking of an ear vein and read immediately using the same glucose meter. Data were analysed by Pearson correlations (IBM SPSS Statistics for Windows, v25.0. Armonk, NY).

Results The umbilical venous-arterial glucose difference was $0.3 \pm 0.45 \mathrm{mM}$ (mean $\pm \mathrm{SD}$ ), and the mean value was similar to the difference measured in catheterised pig foetuses in late gestation (Père, 1995). The venous-arterial glucose difference did not correlate with piglet birth weight $(1.43 \pm 0.38 \mathrm{~kg})$ or the blood glucose concentration of farrowing sows $(5.6 \pm 0.97 \mathrm{mM})$. Glucose concentration in the umbilical vein $(3.2 \pm 0.77 \mathrm{mM})$ and artery $(2.9 \pm 0.85 \mathrm{mM})$ were both positively correlated with glucose concentration in the ear vein of the farrowing sow.

Table 1. Pearson correlation (below diagonal) and significance (above diagonal) of the relationship between glucose concentration in the sow, umbilical glucose concentrations, and piglet birth weight.

\begin{tabular}{lccccc}
\hline & $\begin{array}{c}\text { Sow ear } \\
\text { vein }\end{array}$ & $\begin{array}{c}\text { Umbilical } \\
\text { vein }\end{array}$ & $\begin{array}{c}\text { Umbilical } \\
\text { artery }\end{array}$ & $\begin{array}{c}\text { Venous-arterial } \\
\text { difference }\end{array}$ & $\begin{array}{c}\text { Piglet birth } \\
\text { weight }\end{array}$ \\
\hline Sow ear vein & - & $<\mathbf{0 . 0 0 1}$ & $\mathbf{< . 0 0 1}$ & 0.77 & 0.39 \\
Umbilical vein & 0.45 & - & $<\mathbf{0 . 0 0 1}$ & 0.10 & 0.16 \\
Umbilical artery & 0.39 & 0.83 & - & $<\mathbf{0 . 0 0 1}$ & 0.44 \\
Venous-arterial difference & 0.03 & 0.14 & -0.43 & - & 0.35 \\
Piglet birth weight & -0.08 & -0.12 & -0.07 & -0.08 & - \\
\hline
\end{tabular}

Conclusion Piglet birth weight did not correlate with umbilical venous-arterial glucose difference measured at birth. Since net glucose uptake can be calculated by venous-arterial glucose difference multiplied by umbilical blood flow rate, it appears possible that differences in umbilical blood flow may be more important in determining net glucose uptake and foetal development.

Acknowledgements Supported in part by Australian Pork Limited (Project number 2017/2213) and supported by staff at Research and Innovation Unit and Farming Operations of Rivalea Australia Pty Ltd.

\section{References}

Fowden AL, Forhead AJ, Silver M and MacDonald AA 1997. Experimental Physiology. 82, 171-182.

Hay Jr. WW 1991. In Principles of Perinatal-Neonatal Metabolism (Ed: Cowett RM) Springer-Verlag, New York, USA. pp. 105-171.

Père MC 1995. Journal of Animal Science. 73, 2994-2999.

Sanchez REP, Ochoa GO, Caratachea AJ, Bravo RMR and Rodriguez RO 2016. International Journal of Pure \& Applied Bioscience. 4, 1-7. 
The impact of assistance at farrowing on subsequent reproduction of sows

$\underline{\mathrm{J}} \mathrm{G}$ Alexopoulos $^{1}$ and K J Plush ${ }^{1}$

${ }^{1}$ SunPork Group, Australia

jena.alexopoulos@sunporkfarms.com.au

Application Manual assistance of sows results in a decreased litter size in the subsequent farrowing and so should only be conducted when farrowing distress is evident.

Introduction Up to $8 \%$ of newborn piglets are stillborn. Farrowing supervision leads to high performing herds with lower incidence of stillborn piglets (Vanderhaeghe et al., 2010). Manual assistance during farrowing involves a sterile, lubricated gloved hand inserted internally through the sow's vagina and removing any piglets present. Producers commonly assist a sow when the piglet birth interval exceeds 30-45 minutes. There may be an increased risk of disease transfer into the uterus as well as physical damage resulting in reduced subsequent reproductive performance. Björkman et al. (2017) reported an increase in postpartum metritis as a result of manual assistance which delayed uterine involution. Thus, the aim of this study was to assess whether manual assistance at farrowing impaired the ability of the sow to be rebred successfully. We hypothesised that sows who were manually assisted during farrowing would display reduced farrowing rate and litter size after being rebred.

Methods The study involved 808 sows (parity 1 to 9). At the time of farrowing, manual assistance was provided when a piglet had not been born after 45 minutes or when a stillborn was expelled. Measurements recorded post farrow included; total piglets born (TB), piglets born alive (BA), stillborn piglets (SB) and mummified piglets. Measurements recorded post weaning were wean to service interval (WSI), removals and subsequent reproductive performance (TB, BA, SB and mummified piglets). Data were analysed with shed as the random term, TB from first litter as covariate, parity, farrowing status (overnight or during working hours $(0700-1600 \mathrm{~h}$ ); finished or still going), assistance (yes or no), and the interaction between farrowing status and assistance, using the appropriate statistical model for observed distribution (IBM SPSS Statistics for Windows, v25.0. Armonk, NY).

Results The number of stillborn piglets increased with parity $(P<0.001)$ and litter size $(P<0.001)$. Sows that had completed farrowing prior to the end of the work day had an additional 0.6 piglets born dead when they were unassisted compared to assisted sows $(P=0.001)$. Sows that farrowed overnight had an increased number of stillborn piglets $(P=$ $0.001)$, and sows that were not assisted nor completed farrowing during the working day had 0.2 more stillborn piglets than sows that were assisted $(P=0.001)$. WSI, conception and farrowing rates were not affected by assistance during farrowing (Table 1). The total number of pigs born and number of piglets born alive in the subsequent litter were reduced in sows that were assisted.

Table 1. Mean \pm SEM of subsequent reproductive performance of assisted and not assisted sows

\begin{tabular}{lccc}
\hline & Assisted & Not Assisted & $P$ value \\
\hline WSI & $6.3 \pm 1.4$ & $8.4 \pm 0.70$ & 0.119 \\
Bred $<7 \mathrm{~d} \% *$ & $99 \%(74-100)$ & $84 \%(76-90)$ & 0.930 \\
Pregnancy Rate \%* & $82 \%(70-91)$ & $88 \%(83-99)$ & 0.319 \\
Farrowing Rate \%* & $76 \%(63-79)$ & $76 \%(70-81)$ & 0.773 \\
Total born & $11.3 \pm 0.5$ & $12.7 \pm 0.3$ & 0.016 \\
Born alive & $10.2 \pm 0.5$ & $11.5 \pm 0.3$ & 0.020 \\
Stillborn & $1.5 \pm 0.2$ & $1.4 \pm 0.1$ & 0.689 \\
\hline
\end{tabular}

$* 95 \%$ confidence intervals rather than SEM are presented for binary data; SEM, standard error of the means; WSI, wean to service interval.

Conclusion When a sow is assisted during farrowing, it increases the risk of postpartum metritis, delaying the process of uterine involution (Björkman et al., 2017). This repair process prepares a suitable uterine environment to establish subsequent pregnancy after weaning, and its delay is a common cause for embryo mortality and reproductive failure (Belstra et al., 2004). The current finding of reduced subsequent litter size after manual assistance would support previous work in this area and suggests the intervention should only be applied when extreme farrowing difficulty is observed.

\section{References}

Belstra BA, Flowers WL, Croom WJ, DeGroot J and See MT 2005. Animal Reproduction Science. 85, 131-145.

Björkman S, Oliviero C, Rajala-Schultz PJ, Soede NM and Peltoniemi OA 2017. Theriogenology. 92, 36-44.

Vanderhaeghe C, Dewulf J, De Vliegher S, Papadopoulos GA, de Kruif A and Maes D 2010. Animal Reproduction Science. 120, 78-83. 


\section{The effect of fostering status and nurse sow parity on pre-weaning survival}

$\mathrm{J} \mathrm{Harper}^{1}$ and K L Bunter ${ }^{2}$

${ }^{1}$ Rivalea Australia Pty Ltd, Australia, ${ }^{2}$ Animal Genetics and Breeding Unit, a joint venture of NSW Department of Primary Industries and University of New England, University of New England, Australia.

jharper@rivalea.com.au

Application. Optimising fostering procedures to maximise piglet survival in commercial farrowing houses.

Introduction Fostering is a process by which nursing piglets are moved from one sow to another and is generally performed to even out litter size, matching the number of piglets to be suckled with the number of sow teats available (APL, 2017), typically before two days of age. Other reasons for fostering include when piglets appear to be losing condition, the sow is savaging piglets or is unable to care for the litter, or sow death (Heim et al., 2012) which generally occurs after two days of age. The aim of this study was to investigate the effect of nurse sow parity on the survival of piglets either not fostered or fostered up to or after two days of age.

Material and methods Data on individual piglet deaths ( $0=$ alive, $1=$ dead) before weaning (average $26 \mathrm{~d})$ and other related traits were recorded at a commercial nucleus piggery (Primegro ${ }^{\mathrm{TM}}$ Genetics, Corowa, NSW). Data included 262060 piglets, born to first and second parity sows, individually identified within $24 \mathrm{~h}$ from birth. Cross fostering occurred after identification, and all movements and deaths of individual piglets were recorded. However, nurse sow parity for fostering events after d 2 was not recorded. Logistic regression was performed to analyse individual piglet mortality using the GLM procedure in R (R Core Team 2016), where the fostering-birth-nurse sow parity group effect was investigated using a model accounting for the fixed effects of breed (3 levels), sex (M, F), birthweight class (6 levels), gestation length class (6 levels) and birth year-quarter (40 levels), previously found to be significant $(P<0.0001)$. Probabilities were predicted after log-transformation of the data, and the effect of fostering between birth parity 1 or 2 and nurse parity classes are presented in this paper in terms of survival, as deviations from the overall mean.

Results On average $88.8 \%$ of piglets were nursed by their biological mothers, $7.1 \%$ were first fostered before $\mathrm{d} 2$ of age and a further $4.1 \%$ were first fostered after d 2 (Table 1). Un-fostered piglets had positive survival outcomes, and this included piglets from both large (requiring fostering) and small (not requiring fostering) litters. Progeny fostered before d 2 from a birth to nurse parity 1 sow significantly $(P<0.05)$ increased the chances of piglet survival $(1.74 \pm 0.50 \%)$ compared to fostering to any other nurse parity class. Similarly, progeny born to parity 2 sows fostered before $\mathrm{d} 2$ to parity 1 nurse sows significantly $(P<0.05)$ increased the survival of piglets $(4.94 \pm 0.45 \%)$ compared to fostering to other parity classes, with similar results for non-fostered piglets $(3.94 \pm 0.07 \%)$. Udder deterioration (teat quality and quantity) with increasing parity (Huting et al., 2019) may possibly be contributing to this outcome. Parity 1 progeny fostered after $\mathrm{d} 2$ had reduced survival compared to parity 2 progeny.

Table 1. Percentages \pm SE for piglet survival presented as deviations from the overall survival percentage for piglets not fostered, fostered before d 2 or fostered after d 2, between birth parity 1 or 2 and nurse sow parity

\begin{tabular}{ccccc}
\hline Birth Sow Parity & Nurse Sow Parity & Not fostered & Fostered before d 2 & Fostered after d 2 \\
\hline 1 & 1 & $0.14 \pm 0.08$ & $1.74 \pm 0.50$ & $-8.78 \pm 0.35$ \\
2 & & $-3.57 \pm 0.69$ & \\
$3-5$ & & $-5.37 \pm 0.77$ & \\
$6+$ & & $-3.47 \pm 1.71$ & \\
2 & 1 & $4.94 \pm 0.45$ & $0.84 \pm 0.44$ \\
& $3-5$ & $3.94 \pm 0.07$ & $1.74 \pm 0.48$ & \\
& $6+$ & & $-1.37 \pm 0.55$ & \\
\hline
\end{tabular}

Conclusion Progeny of early parity sows (parities 1 and 2) requiring fostering due to large litter size should be cross fostered to early parity nurse sows to maximise their survival. These results warrant further investigation into the patterns of survival of progeny from later parity sows, which could be implemented into farrowing house cross fostering procedures to maximise piglet survival.

Acknowledgements Supported by Rivalea Australia Pty Ltd.

\section{References}

APL 2017 APL project 2016/2237, Cross fostering guidelines for Australian farrowing houses, 2017.

Heim G, Mellagi APG, Bierhals T, deSouza LP, deFries HCC, Piuco P, Seidel E, Bernardi ML, Wentz I and Bortolozzo FP 2012 Livestock Science 150, 121-127.

Huting AMS, Sakkas P and Kyriazakis I 2019. Journal of Animal Science. 97, 1656-1670.

R Core Team 2016. R: A language and environment for statistical computing. R Foundation for Statistical Computing, Vienna, Austria. 
Can we predict shortened or prolonged gestation upon transfer to the farrowing shed?

$L_{\text {L Vargovic }}^{1}$, K L Bunter ${ }^{1}$, S Hermesch ${ }^{1}$, P C Condous ${ }^{2}$, J Harper ${ }^{3}$

${ }^{I}$ Animal Genetics and Breeding Unit, a joint venture of NSW Department of Primary Industries and University of New England, University of New England, Australia, ${ }^{2}$ SunPork Group, Australia, ${ }^{3}$ Rivalea Australia Pty Ltd, Australia lvargovi@myune.edu.au

Application Results from this research could provide an opportunity to predict shortened or prolonged gestation, which both have direct implications for health, welfare and productivity of both sows and piglets.

Introduction Farrowing is the most critical period for the survival of both sows and piglets. Farrowing is considered successful if completed within $5 \mathrm{~h}$, with more than $90 \%$ of born alive piglets surviving the following $72 \mathrm{~h}$, otherwise there is increased risk of stillborns, higher pre-weaning mortality or health implications for the sow (Peltoniemi and Oliviero, 2015). A better understanding of the timing of farrowing could help to allocate labour to increase observation during the critical days and therefore improve farrowing results (Peltoniemi and Oliviero, 2015). Preliminary analyses showed that approximately $92 \%$ of sows farrowed within $2 \mathrm{~d}$ of the expected date, but there was no improvement in overall prediction capacity from observing other factors. This study tested the hypothesis that individual observations for sows upon the transfer to the farrowing shed could be used to better predict either shortened or prolonged gestations.

Material and methods Data were available for 1100 sows recorded at 2 nucleus farms located in NSW, Australia. Sows were scored for a range of attributes upon the entry to the farrowing house, including: rectal temperature scored as $0=$ normal and $1=$ elevated $\left(>38.6^{\circ} \mathrm{C}\right)$; count of injured teats, scored from 0 (no teats injured) to 3 (3+ teats injured); feed refusal observed on the day after the transfer as $0=$ feed eaten or $1=$ feed refused; treatment during gestation scored as $0=$ not treated or $1=$ treated; body condition score assessed by caliper increments (Knauer and Baitinger, 2015), Score 0: $<11 ; 1$ : [11-12]; 2: [13-14]; 3: [15-16] and 4: >16; injuries to the vulva with increasing severity scored 0-2; and udder and vulva development scores, with increasing development scored $0-2$. Sows unrecorded for an attribute were assigned to a separate class level. Premature or prolonged gestation outcomes were considered present if the gestation length was $<114$ $\mathrm{d}$ or $>118 \mathrm{~d}$. Logistic regression was performed to analyse the outcome data using the GLM procedure in R 3.5.1 (R Core Team, 2018)). The basic model for shortened gestation included breed group, farm and parity group. The basic model accounted for breed group (maternal vs terminal) nested within farm, and parity group (gilts: 1 vs sows: 2 ) for prolonged gestation. Differences between levels were determined by using Tukey test with $P<0.05$ indicating significance.

Results Sows with pre-farrowing fever, with teat injuries, prior health related treatment and feed refusal were at increased risk of premature farrowing (Table 1). Maternal sows were at higher risk of premature farrowing and terminal sows were at higher risk of prolonged gestation (not shown). Prolonged gestation was associated with an under-developed udder at entry, absence of injuries or moderate vulva development scores.

Table 1. Least square means $\pm S E$ for the percentage of sows with shortened $(\mathrm{N}=31)$ or prolonged $(\mathrm{N}=55)$ gestation by class of predictor (mean gestation length $=116.2 \mathrm{~d}$ )

\begin{tabular}{|c|c|c|c|c|c|c|}
\hline \multirow[b]{2}{*}{ Predictors } & \multicolumn{5}{|c|}{ Percentage of sows with gestation $<114$ days $(2.82 \%$ of sows $)$} & \multirow[b]{2}{*}{$P$ value } \\
\hline & 0 & 1 & 2 & 3 & 4 & \\
\hline Rectal temperature & $1.07^{\mathrm{a}} \pm 0.37$ & $7.22^{\mathrm{b}} \pm 4.41$ & & & $* 1.92^{\mathrm{ab}} \pm 1.59$ & 0.029 \\
\hline Teat injuries & $0.93^{\mathrm{a}} \pm 0.37$ & $0.88^{\mathrm{a}} \pm 0.48$ & $4.15^{\mathrm{b}} \pm 1.75$ & $2.20^{\mathrm{ab}} \pm 1.47$ & & 0.012 \\
\hline Day 1 feed refusal & $1.04^{\mathrm{a}} \pm 0.36$ & $2.29^{\mathrm{a}} \pm 1.09$ & & & & 0.10 \\
\hline Treatment gestation & $1.11^{\mathrm{a}} \pm 0.38$ & $3.69^{\mathrm{a}} \pm 2.44$ & & & & 0.081 \\
\hline Caliper increments & $0.96^{\mathrm{a}} \pm 0.79$ & $1.52^{\mathrm{a}} \pm 0.78$ & $2.29^{\mathrm{a}} \pm 0.84$ & $0.55^{\mathrm{a}} \pm 0.35$ & $0.90^{\mathrm{a}} \pm 0.57$ & 0.11 \\
\hline \multicolumn{7}{|c|}{ Percentage of sows with gestation $>118$ days $(5.0 \%$ of sows $)$} \\
\hline Injuries vulva & $5.03^{\mathrm{a}} \pm 0.87$ & $1.87^{\mathrm{a}} \pm 0.80$ & $2.06^{\mathrm{a}} \pm 1.23$ & & & 0.025 \\
\hline Udder score & $14.7^{\mathrm{a}} \pm 4.29$ & $4.30^{\mathrm{b}} \pm 0.92$ & $1.71^{\mathrm{c}} \pm 0.60$ & & & $<0.0001$ \\
\hline Vulva score & $2.08^{\mathrm{a}} \pm 0.71$ & $5.22^{\mathrm{b}} \pm 0.96$ & $2.23^{\mathrm{ab}} \pm 2.24$ & & & 0.029 \\
\hline
\end{tabular}

Conclusion These results suggest that some pre-farrowing attributes (in particular related to sow health) were associated with shortened or prolonged gestation, which provides opportunities for improving management of the farrowing event.

Acknowledgements Supported in part by Pork CRC Limited (Project 2A-116) and the UNE via International Postgraduate Research Award (UNE IPRA).

\section{References}

Knauer MT and Baitinger DJ 2015. Applied Engineering in Agriculture. 31, 175-178.

Peltoniemi $\mathrm{O}$ and Oliviero C 2015. In The gestating and lactating sow (Ed: Farmer C), Wageningen Academic Publisher, the Netherlands. pp. 231-252.

R Core Team (2018). R: A language and environment for statistical computing. R Foundation for Statistical Computing, Vienna, Austria. 


\section{Thermal imaging of skin but not eye can be used as an alternative body temperature measurement in lactating sows in a hot environment.}

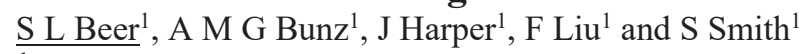

${ }^{1}$ Rivalea Australia Pty Ltd, Australia

sbeer@rivalea.com.au

Application Thermal imaged skin temperatures taken on sows at days 15 and 21 during lactation were moderately correlated to rectal temperatures and respiration rates. This indicates a potential to use thermal imaging as a simple and less invasive measure of sow body temperature and heat stress.

Introduction Respiration rate and rectal temperature are traditionally used to assess sow body temperature and can be an indication of heat stress (Gourdine et al., 2017). These measures can be subjective when performed by multiple stock people and are also invasive and disruptive to the sow. Thermal imaging has been used recently for injury detection in domestic animals as well as animal counts and distributions in wild populations (Havens and Sharp, 2015) but has limited reported use in sow body temperature estimation. This study tested the hypothesis that thermal imaging of either eye or skin, a less intrusive and labour-intensive method, would be a suitable alternative to standard rectal temperature and respiration rate measures of lactating sows under hot conditions.

Material and methods Thermal physiology measurements were collected from 103 parity 2 and 3, pure and crossbred line sows (Large White, Landrace and Large White $\mathrm{x}$ Landrace, Primegro ${ }^{\mathrm{TM}}$ Genetics, Corowa, NSW) during lactation. All sows in each of the three replicates were measured on the same day at the average d 3, 14 and 20 of lactation. The study was conducted over summer months (January to February 2019) at approximately $1500-1700 \mathrm{~h}$ when ambient temperatures were expected to be approaching their daily peak. The average daily maximum temperature over the study was $34.5^{\circ} \mathrm{C}$. Skin and eye temperature were collected using an FLIR thermal imaging camera (FLIR Systems Inc., Tallin, Estonia), with skin temperature being recorded from the hottest point of the sows' shoulder and eye temperature from the centre of the sows' open eye. Measurements were not taken where skin was damp, soiled or injured and the camera was held at a consistent distance from each sow to obtain the most accurate temperatures. Rectal temperature was measured using a digital thermometer and respiration rate was counted as breaths/minute. Pearson's correlation coefficient was obtained to measure the association between standard and novel temperature measurements for all breeds over three time points in lactation (Table 1., R Core Team 2018).

Results Rectal temperatures were moderately correlated $(r \geq 0.33)$ with skin temperatures, particularly at $d 14$ post farrowing where sows were found to have generally higher rectal temperatures (Table 1). Eye temperature was less well correlated to rectal temperature $(r \geq 0.28)$ and was poorly correlated to respiration rates at all time points. This suggests that eye temperature would not be a suitable substitute measurement of body temperature contrary to the hypothesis.

Table 1. Correlations (r) between standard (respiration rate, RR and rectal temperature, $\mathrm{RT}$ ) and thermal imaging measurements of skin and eye temperature for all breeds over three time points in lactation. In addition, mean \pm SD for $\mathrm{RR}$ (breaths/minute) and $\mathrm{RT}\left({ }^{\circ} \mathrm{C}\right)$ at all 3 time points.

\begin{tabular}{lcccccc}
\hline & \multicolumn{3}{c}{$\mathrm{d} 3$} & \multicolumn{2}{c}{$\mathrm{d} 14$} & $\mathrm{~d} 20$ \\
& $\mathrm{RR}$ & $\mathrm{RT}$ & $\mathrm{RR}$ & $\mathrm{RT}$ & $\mathrm{RR}$ & $\mathrm{RT}$ \\
\hline & $44.2 \pm 33.1$ & $39.2 \pm 0.58$ & $57.3 \pm 32.0$ & $39.2 \pm 0.79$ & $35.6 \pm 25.8$ & $39.0 \pm 0.63$ \\
$\mathrm{RR}$ & - & $0.46^{*}$ & - & $0.63^{*}$ & - & $0.21^{*}$ \\
$\mathrm{RT}$ & $0.46^{*}$ & - & $0.63^{*}$ & - & $0.21^{*}$ & - \\
Eye & 0.04 & 0.48 & 0.09 & $0.27^{*}$ & 0.08 & $0.33^{*}$ \\
Skin & 0.07 & 0.33 & $0.57^{*}$ & $0.66^{*}$ & $0.43^{*}$ & $0.51^{*}$ \\
\hline \multicolumn{7}{r}{}
\end{tabular}

*indicates Pearson's correlation being significantly different from $0(P<0.05)$

Conclusion The correlations showed that thermal imaged measurement of skin temperature has the potential to be used by stock people as an easy to use alternative for measurement of body temperature in lactating sows. At time points $d 14$ and 20 this measurement would be a suitable substitute for respiration rate and rectal temperature. Further investigation is warranted to look at the association with body temperature as measured by thermal imaging techniques to subsequent reproduction of sows.

Acknowledgements Supported by staff at Research and Innovation Unit and Farming Operations at Rivalea Australia Pty Ltd.

\section{References}

Gourdine J, Mandonnet N, Giorgi M and Renaudeau D 2017. Animal. 11, 365-374.

Havens KJ and Sharp EJ 2015. Thermal imaging techniques to survey and monitor animals in the wild: a methodology. Academic Press, Cambridge, MA, USA.

R Core Team (2018). R: A language and environment for statistical computing. R Foundation for Statistical Computing, Vienna, Austria. 


\section{Risk-based assessment of criteria used for disposition judgement for polyarthritis of pigs}

A M Pointon $^{1}$, D R Hamilton ${ }^{2}$, S Lester ${ }^{1}$ and A Kiermeier ${ }^{3}$

${ }^{1}$ APFoodIntegrity Pty Ltd, Australia, ${ }^{2}$ D Hamilton Consulting Pty Ltd, Australia, ${ }^{3}$ Statistical Process Improvement Consulting \& Training Pty Ltd, Australia

andypointon.food@iinet.com.au

Application The alternative criteria approved for disposition judgement of arthritic carcasses minimises undue wastage of those with multiple chronic lesions while verifying wholesomeness of meat for consumption.

Introduction The review of the Australian Standard AS4696 (Standards Australia, 2007) undertook risk-based assessments of criteria used to determine final carcass disposition judgement (Pointon et al., 2018). The leading reason of total carcass condemnation of pig carcasses over recent years is arthritis/polyarthritis (Pointon et al., 2018). While arthritis is classified as acute or chronic in the Australian Standard, anecdotal evidence is that disposition judgements based on involvement of more than one joint alone may be used to determine "systemic effects" resulting in total carcass condemnation, when without signs of septicaemia and/or cachexia. As infectious arthritis results from systemic infections, the aim of inspection is to determine whether the carcass has progressed in the continuum from acute, to resolving, to chronic. The aim of this investigation was to validate criteria to minimise uncertainty in determining final carcass disposition.

Materials and methods Pig carcasses with polyarthritis at two export licenced abattoirs were investigated. The case definition was having a final carcass disposition of total condemnation due to polyarthritis as the primary reason (i.e. multiple joints affected). The risk-based assessment of the disposition criteria was based on the approach of Kruse et al. (2015). Unopened, severely affected joints were submitted for bacteriological investigation that included:

- Assessment of the acute (current systemic) versus chronic (localised) nature of gross abnormalities;

- Determination if the carcass is septicaemic or infection is localised i.e. whether likely primary or secondary agents were isolated from affected joints, lymph nodes not directly draining the abnormality and edible tissue;

- Assessment of the presence or absence of food safety hazards in edible tissue.

Testing for Salmonella spp. as a potential cause of septicaemia or sub-clinical bacteraemia as a sequalae to polyarthritis was included to inform the food safety status of edible tissue. Full methods are reported by Pointon et al. (2017).

Results Twenty eight pigs having 19 separate production entities were examined. The main findings included:

- isolates from twenty joints included Erysipelothrix rhusiopathiae (9 positive), Streptococcus suis (9), Trueperella pyogenes (1) and Streptococcus disgalacticae (1);

- these bacteria were not isolated from peripheral lymph nodes or edible tissues of the corrresponding carcass;

- similarly, foodborne hazards were not isolated from these same peripheral lymph nodes or edible tissues;

- a positive trend toward pleurisy being associated with carcasses from which Streptococcus suis was isolated from joints $(P=0.096)$, while the opposite was significant for Erysipelothrix rhusiopathiae $(P=0.046)$.

Conclusions The similar isolation of Erysipelothrix rhusiopathiae and Streptococcus suis is consistent with findings of previous investigations of polyarthritis (Pointon et al., 2018). The pattern of isolation of infectious agents was consistent with chronic (localised) stage of disease in carcasses meeting the case definition (Pointon et al., 2017). Carcass wholesomeness for consumption can be achieved by removing multiple chronically affected joints. As most joint isolates were Erysipelothrix rhusiopathiae and Streptococcus suis, which are occupational zoonoses, not incising arthritic joints at routine inspection is supported to avoid oocupational exposure to zoonotic hazards. Total carcass condemnation should apply when there is gross evidence of acute systemic infection (i.e. septicaemia indicated by petechial haemorrhage, polyserositis, generalised erythema). Consequently, to minimise uncertainty in judging final carcass disposition alternative wording now includes 1) acute with specific signs of septicaemia as described here, 2) "systemic effects" being deleted and 3) carcasses with multiple chronic abnormalities may be trimmed instead of being totally condemned when not showing signs of cachexia i.e. body score 1 or possibly 2 (APL, 2016; Pointon et al., 2017). This alternative disposition criteria description has been approved for the amendment of Schedule 3 of the Australian Standard and is being implemented (Standards Australia, 2019).

Acknowledgements Supported in part by Australian Pork Limited and the Australian Meat Processors Corporation. Abattoir personnel who collected the affected joints for laboratory examination are thanked for their assistance.

\section{References}

APL 2016 Is it fit for the intended journey? 2nd Edition. Australian Pork Ltd, Barton, ACT.

http://australianpork.com.au/wp-content/uploads/2018/08/FFTIJ-A-Guide-Final-Document.pdf

Kruse AB, Larsen MH, Skou PB and Alban L 2015. International Journal of Food Microbiology. 196, 32-39.

Pointon AM, Hamilton D and Kiermeier A 2017. APL Final Report 2015/023. Australian Pork Ltd, Barton, ACT.

Pointon AM, Hamilton D and Kiermeier A 2018. Food Control. 90, 222-232.

Standards Australia 2007. Food Regulation Standing Committee Technical Report Series 3. AS 4696:2007. Standards Australia, Sydney, NSW.

Standards Australia 2019. Amended AS 4696:2007. Standards Australia, Sydney, NSW. In Press. 


\section{Validation of alternative post-mortem inspection procedures with reference to pleurisy of pigs} in Australia

A M Pointon $^{1}$, D R Hamilton ${ }^{2}$ and A Kiermeier ${ }^{3}$

${ }^{1}$ APFoodIntegrity Pty Ltd, Australia, ${ }^{2}$ D Hamilton Consulting Pty Ltd, Australia, ${ }^{3}$ Statistical Process Improvement Consulting \& Training Pty Ltd, Australia

andypointon.food@iinet.com.au

Application The detection and partial trimming ("lifting") of pleuritic carcasses by slaughterline staff before official inspection is very sensitive. This enables a reduction in carcasses diverted to the retain rail while preserving information on gross abnormalities needed for official carcass disposition judgement. These findings enable feasible and acceptable improvement in efficiency of processing and official inspection.

Introduction The terms of reference of the review of post-mortem inspection included assessing where appropriate, the transfer of procedures that are principally related to product quality rather than food safety, to companies' quality assurance systems (Pointon et al., 2018). Pleurisy is a leading reason for partial trimming and total carcass condemnation in pigs (Hudson and Hamiton, 2016) and can lead to processing inefficiency due to excessive numbers of carcasses needing to be railed-off for trimming. This report quantifies the effectiveness of the detection of pleurisy as part of routine carcass dressing by slaughterline staff.

Materials and methods Due to the relatively high prevalence of pleurisy, in-plant validation of the detection of adhesions/diffuse pleurisy by company staff was feasible and conducted at one abattoir. A team of three observers (the authors) recorded pleurisy data on the same carcasses at the point of routine thoracic organ evisceration (Stage 1: Lifting), official inspection (Stage 2: Inspection) and pre-chill (Stage 3). When pleurisy was detected by slaughterline staff, lungs and hearts were removed and the pleura was separated from the thoracic wall (i.e. lifted) and left in situ for official inspection. The assessment also targeted lines of pigs from sources that had higher rates of pleurisy in recent lots. Assessment was done by comparing detection results on the same matched carcasses (i.e. sensitivity of company detection against routine inspection), and recording the residual non-detection rates of combined inspection methods to determine overall sensitivity of both detection methods. Full methods are reported by Pointon et al. (2017).

Results Two thousand three hundred carcasses were numbered and examined at Stages 1, 2 and 3 over three days. The pleurisy prevalence of trial pigs was $26.9 \%$ (619 of 2300 carcasses) of which $86.7 \%$ (95\% CI: $79.2-85.2 \%$; 537 of 619 +ve carcasses) were correctly identified at Stage 1 "Lifting" by the company staff, i.e. sensitivity of company staff for detection of pleurisy.

Table 1. Detection of pleurisy by "lifters" and official inspectors on an individual carcass basis

\begin{tabular}{lccc}
\hline & \multicolumn{2}{c}{ Stage 2: Inspection } & Total \\
\hline Stage 1: Lifting & Correctly identified at Stage 1 & Pleurisy missed at Stage 1 & 1748 \\
No pleurisy & 1681 & 67 & 552 \\
Pleurisy (1 or 2 sides) & 537 & $15^{*}$ & 2300 \\
Total & 2218 & 82 & 2300 he been
\end{tabular}
affected at Stage 2 .

Conclusions The procedure results in a high detection rate of pleurisy cases by "lifters" enabling preliminary carcass trimming (i.e. "lifting" pleura) to achieve carcass wholesomeness without being detained on the retail rail. In addition, there is no loss of information to inform final carcass disposition judgement as "lifted" pleura are obvious to official carcass inspectors. These findings offer feasible improvement in efficiency for both processing and official inspection.

While the sensitivity of detection of pleurisy by "lifters" was high, there remains opportunity for improvement. Reasons for missed cases by "lifters" include 1) mild cases that may be undetected 2) lighting at the Stage 1 "lifting" point not equivalent to that provided for official inspection 3) occasional distraction of "lifters" is more likely as is not an official procedure and 4) insufficient "lifter" capacity at times of unpredicted high prevalence in some lots. As a result the sensitivity of detection by "lifters" recorded in this assessment is likely to represent a worst-case outcome. These observations provide optimism that investment to minimise these may further improve detection rate.

Acknowledgements Supported in part by Australian Pork Limited and the Australian Meat Processors Corporation. Abattoir management that hosted the project are thanked for their assistance.

\section{References}

Hudson D and Hamilton D 2016. APL Final Report 2015/2209. Australian Pork Ltd, Barton, ACT.

Pointon A, Hamilton D and Kiermeier A 2017. APL Final Report 2015/023. Australian Pork Ltd, Barton, ACT.

Pointon A, Hamilton D and Kiermeier A 2018. Food Control. 90, 222-232. 


\section{Dietary supplementation of a Bacillus licheniformis derived-protease, alone or in combination with valine to a lower protein diet improves growth performance in pigs}

$\mathrm{J} \mathrm{Hu}^{1}$, L Cheng ${ }^{1}$ and $\mathrm{I} \mathrm{H} \mathrm{Kim}^{1}$

${ }^{1}$ Dankook University, Republic of Korea

inhokim@dankook.ac.kr

Application Bacillus licheniformis derived-protease in combination with valine may improve performance in pigs fed lower protein diets.

Introduction The price of feed grains (especially corn and soybean meal) are dramatically increasing due to the surge in demand by its inclusion as the dominant feed ingredient in animal production and increasing human consumption. This has reduced the profit margins of pork production (Niemi et al., 2010). The requirements of protein-rich feed ingredients in the swine industry are essentially a need for the availability of amino acids (Gloaguen et al., 2014). Addition of Bacillus licheniformis derived-protease has potential to increase protein utilisation, which could exert beneficial effects on growth performance and nutrient digestibility in finishing pigs fed lower protein diets (Lei et al., 2017; Nguyen et al., 2018). Moreover, a lower protein diet supplemented with valine has also resulted in improved performance of growing pigs (Liu et al., 2015; Soumeh et al., 2015). Therefore, we hypothesised that the inclusion of a Bacillus licheniformis derived-protease in combination with valine to lower protein diets will increase growth performance in growing-finishing pigs.

Material and methods One-hundred and eighty crossbred (Landrace x Yorkshire $\mathrm{x}$ Duroc) growing pigs with an initial body weight of $22.56 \pm 1.59 \mathrm{~kg}$ were used in a 17 week feeding trial, with a 6 week growing phase and an 11 week finshing phase. Pigs were randomly allotted into 3 treatments: CON, basal diet (growing and finishing diets containing $17.56 \%$, and $15.89 \%$ crude protein, respectively); PROT, lower protein diet (growing, and finishing diets containing $16.83 \%$, and $15.15 \%$ crude protein, respectively) with $0.05 \%$ protease; PROTV, PROT diet with $0.08 \%$ Valine. The basal diet contained $10.35 \mathrm{MJ}$ digestible energy $/ \mathrm{kg}, 0.99$ and $0.89 \%$ of standard ileal digestible lysine for growing and finishing pigs, respectively. All data were analyzed using the mixed procedure in SAS 9.4 (SAS Institute Inc., Cary, NC, USA). Differences among treatment means were determined using the Duncan's multiple range test with a $P<0.05$ indicating significance.

Results During the finishing phase (wk 7 to 17) and across the overall period (wk 0 to 17) gain:feed ratio was increased $(P<0.05)$ in pigs fed PROTV diet compared with those fed CON diet. No other growth measure was affected by treatment.

Table 1. Effect of $0.05 \%$ protease alone (PROT), and in combination with $0.08 \%$ valine supplementation (PROTV) in lower protein diets on growth performance in growing-finishing pigs.

\begin{tabular}{cccccc}
\hline & CON & PROT & PROTV & SEM & $P$ value \\
\hline Growing phase (week 0 to 6) & & & & & 0.312 \\
ADG (g/d) & 709 & 723 & 729 & 9 & 0.500 \\
ADFI (g/d) & 1600 & 1632 & 1625 & 20 & 0.377 \\
G:F (g/g) & 0.443 & 0.443 & 0.449 & 0.003 & 0.297 \\
Finishing phase (week 7 to 17) & 794 & 803 & 822 & 13 & 0.810 \\
ADG (g/d) & 2664 & 2631 & 2655 & 37 & 0.011 \\
G:F (g/g) & $0.298^{\mathrm{b}}$ & $0.305^{\mathrm{ab}}$ & $0.310^{\mathrm{a}}$ & 0.002 & 0.291 \\
Overall experiment & & & & & 0.948 \\
ADG (g/d) & 764 & 775 & 789 & 29 & 0.010 \\
ADFI (g/d) & 2289 & 2278 & 2291 & 0.002 & \\
G:F (g/g) & $0.334^{\mathrm{b}}$ & $0.340^{\mathrm{ab}}$ & $0.345^{\mathrm{a}}$ & & \\
\hline
\end{tabular}

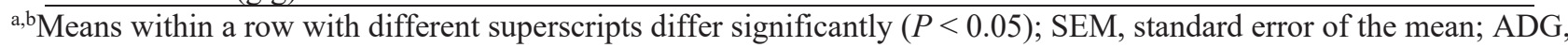
average daily gain; ADFI, average daily feed intake; G:F, gain to feed ratio.

Conclusions Dietary supplementation with combination of protease and valine in low crude protein diet had no deleterious effects on growth performance and improved efficiency in growing-finishing pigs.

\section{References}

Gloaguen M, Le Floc'h N, Corrent E, Primot Y and van Milgen J 2014. Journal of Animal Science. 92, 637-644.

Lei XJ, Cheong JY, Park JH and Kim IH 2017. Animal Science Journal. 88, 1987-1993.

Liu XT, Ma WF, Zeng XF, Xie CY, Thacker PA, Htoo JK and Qiao SY 2015. Journal of Animal Science. 93, 47614773.

Nguyen DH, Lee SI, Cheong JY and Kim IH 2018. Canadian Journal of Animal Science. 98, 488-497.

Niemi JK, Sevón-Aimonen ML, Pietola K and Stalder KJ 2010. Livestock Science. 129, 13-23.

Soumeh EA, van Milgen J, Sloth NM, Corrent E, Poulsen HD and Nørgaard JV 2015. Animal. 9, 1312-1318. 


\section{Effects of low dose $\mathrm{ZnO}$ combined with probiotic complex relative to high dose $\mathrm{ZnO}$ on the performance, nutrient digestibility and faecal microbial counts in weaner pigs}

S D Upadhaya ${ }^{1}, \mathrm{H} \mathrm{Shi}^{1}$, $\mathrm{J} \mathrm{Hu}^{1}$ and I H Kim${ }^{1}$

${ }^{I}$ Dankook University, Republic of Korea

inhokim@dankook.ac.kr

Application The pharmacological dose of zinc oxide $(\mathrm{ZnO})$ used for the prevention of diarrhoea in weaned pigs has adverse environmental effects due to faecal zinc $(\mathrm{Zn})$ excretion. Combination of a lower level of $\mathrm{ZnO}$ with probiotic could be a viable strategy to reduce $\mathrm{ZnO}$ dose while minimizing weaning stress and diarrhoea and faecal $\mathrm{Zn}$ excretion.

Introduction For the control of diarrhoea and to enhance growth performance in weaned pigs a pharmacological dose of $\mathrm{ZnO}$ (3 $000 \mathrm{ppm}$ ) has been practiced (Milani et al., 2017). However, a large percentage of this $\mathrm{Zn}$ has been reported to be excreted into the environment (Buff et al., 2005) causing environmental pollution. The objective of this study was to assess the effects of low dose zinc oxide supplemented without or with $0.1 \%$ probiotic complex compared with pharmacological dose $\mathrm{ZnO}$ on the performance, digestibility, blood metabolites, faecal microbial counts and gas emission in weaned piglets. We hypothesized that the combination of low dose $\mathrm{ZnO}$ with probiotic complex may have synergistic effects to improve performance in weaned pigs and reduce $\mathrm{Zn}$ excretion.

Material and methods One hundred and twenty crossbred weaned pigs ((Yorkshire x Landrace) x Duroc, $28 \mathrm{~d}, 7.90$ $\pm 1.20 \mathrm{~kg}$ ) were used in a 6 week experiment. Pigs were blocked by bodyweight (BW) and randomly allocated to 1 of 3 dietary treatments ( 8 replicate pens per treatment, 5 pigs/pen). Treatments consisted of a basal diet supplemented with 3 $000 \mathrm{ppm} \mathrm{ZnO}$ ( $\mathrm{ZH}$, positive control), basal diet supplemented with $300 \mathrm{ppm} \mathrm{ZnO}$ without (ZL) or with $0.1 \%$ probiotic complex (ZLP). All nutrients in diets were formulated to meet or exceed NRC (2012). The commercial probiotic complex contained at least $1 \times 10^{12} \mathrm{CFU} / \mathrm{kg}$ of $B$. coagulans, $5 \times 10^{11} \mathrm{CFU} / \mathrm{kg}$ of B. licheniformis, $1 \times 10^{12} \mathrm{CFU} / \mathrm{kg}$ of $B$. subtilis and $1 \times 10^{11} \mathrm{CFU} / \mathrm{kg}$ of C. butyricum (SynerZymeH10, SynerBig Co. Ltd, Seoul, Republic of Korea). Individual BW and feed consumption was measured weekly to determine average daily gain (ADG), feed intake and gain:feed. Blood sampling, for assessing blood urea nitrogen, creatinine and glucose as well as sampling for nutrient digestibility and faecal microbial counts was undertaken at the end of the experiment (d 42). Data were analysed using the GLM procedure of SAS (SAS Institute Inc., Cary, NC, USA) with pen as the experimental unit. The main effects of ZnO levels (High $v s$ Low), ZH (as positive control) vs ZLP.

Results The BW of piglets fed $\mathrm{ZH}$ diet at the end of the experiment, and the ADG during week 6 and overall were higher $(P<0.05)$ compared with those fed ZL diet. The nutrient digestibility, blood profile and faecal E.coli and Lactobacillus counts were unaffected between $\mathrm{ZL}$ and $\mathrm{ZH}$ diets. The supplementation of probiotic complex to ZL diet increased the ADG during week 6 and overall making it comparable to ZH diet. Otherwise, no other parameters were significantly affected by the inclusion of the probiotic complex to ZL diet compared with ZH diet.

Table 1. Mean growth performance of weaner pigs fed diets containing $3000 \mathrm{ppm} \mathrm{ZnO} \mathrm{(ZH),} 300 \mathrm{ppm} \mathrm{ZnO} \mathrm{(ZL)} \mathrm{or}$ $300 \mathrm{ppm} \mathrm{ZnO}$ plus a probiotic complex (ZLP) across a $42 \mathrm{~d}$ period.

\begin{tabular}{ccccccc}
\hline & & & \multicolumn{3}{c}{$P$ value } \\
& ZH & ZL & ZLP & SEM & ZH vs ZL & ZH vs ZLP \\
\hline Week 6 performance (d 36-d 42) & & & & & \\
Weight (kg) & 25.2 & 24.5 & 24.9 & 0.224 & 0.047 & 0.411 \\
ADG (g/d) & 575 & 546 & 572 & 8.8 & 0.035 & 0.806 \\
ADF (g/d) & 779 & 750 & 784 & 33.3 & 0.554 & 0.905 \\
G/F (g/g) & 0.749 & 0.736 & 0.754 & 0.029 & 0.816 & 0.862 \\
Overall performance (d 0- d 42) & & & & & \\
ADG (g/d) & 411 & 396 & 405 & 5.4 & 0.046 & 0.416 \\
ADF (g/d) & 503 & 487 & 499 & 10.8 & 0.311 & 0.809 \\
G/F (g/g) & 0.820 & 0.815 & 0.819 & 0.019 & 0.785 & 0.927 \\
\hline
\end{tabular}

Conclusions High $\mathrm{ZnO}$ administration exhibited significant effects on $\mathrm{ADG}$ at week 6 compared with low $\mathrm{ZnO}$. However, other parameters, remained unaffected at week 6 . The supplementation of probiotic complex to ZL diet increased the ADG during week 6 and overall making it comparable to ZH diet. The results suggest further research with differing levels of probiotic complex and observation during the early post weaning period is required to reduce $\mathrm{ZnO}$ and maintain performance.

\section{References}

Buff CE, Bollinger DW, Ellersieck MR, Brommelsiek WA and Veum TL 2005. Journal of Animal Science. 83, 23802386.

Milani, NC, Sbardella M, Ikeda NY, Arno A, Mascarenhas BS and Miyada VS 2017. Animal Feed Science and Technology. 227, 13-23.

NRC 2012. Nutrient Requirements of Swine: $11^{\text {th }}$ Revised Edition. The National Academies Press, Washington DC, USA. 


\section{Dexamethasone administrations can reduce intestinal permeability in weaner pigs exposed to mixing stress}

$\underline{\text { S O Sterndale }}^{1}$, D W Miller ${ }^{1}$, J P Mansfield ${ }^{1}$, J C Kim ${ }^{1,2}$ and J R Pluske ${ }^{1}$

${ }^{1}$ Murdoch University, Australia, ${ }^{2}$ AB Vista, Inc., UK

samantha@sterndale.com

Application Administration of a corticosteroid, in this case dexamethasone can reduce intestinal permeability in weaner pigs exposed to an acute stressor such as mixing.

Introduction Weaning involves abrupt separation from the sow, mixing of unfamiliar pigs and change in diet. Psychological stressors have been shown to cause degeneration of intestinal structure, leading to stress-induced villous atrophy and compromised intestinal barrier function (Mereu et al., 2015). Mannitol and Cobalt have been used as markers in plasma to measure gastrointestinal permeability via tight junctions in the enterocytes of the gastrointestinal tract (GIT) (Mereu et al., 2015). Dexamethasone (DEX) is a corticosteroid with anti-inflammatory properties that can reduce the negative effects associated with gastrointestinal tract (GIT) inflammation (Wooten et al., 2019). The hypothesis tested in this study was that DEX administration in weaner pigs exposed to an acute stressor would ameliorate gastrointestinal malfunction.

Material and methods At weaning (d 0), 96 male piglets $(6.5 \pm 0.9 \mathrm{~kg})$ were allocated into pens with their litter mates (4/pen) and allowed to acclimatise for $14 \mathrm{~d}$. At d 14 of the study, pigs were allocated to a 2 x 2 factorial design with the factors being without/with mixing of non-littermates, and without/with DEX supplementation. The study finished on $\mathrm{d}$ 28. On d 12 and 13, pigs in the DEX treatments were given intramuscular (i.m.) injections of $0.2 \mathrm{mg} / \mathrm{kg} \mathrm{DEX} \mathrm{(5} \mathrm{mg/mL,}$ Dexapent, Troy Illium, NSW, Australia) and control pigs were given the same volume of saline i.m. at 0600 and $1800 \mathrm{~h}$ (total 4 doses/pig). On d 14 at 0800 h, 48 pigs were mixed with non-litter mates and 48 pigs remained with their littermates. Four pigs per treatment group $(\mathrm{n}=16)$ were subjected to a GIT absorption test on d 14 and 16. Pigs were given an oral gastric dose with a solution containing $2.5 \mathrm{~mL} / \mathrm{kg} 20 \%$ mannitol (Turpin et al., 2016) (Sigma-Aldrich, Madrid, Spain) and $0.036 \mathrm{~g} / \mathrm{kg}$ Co-EDTA (Udén et al., 1980). Blood samples were collected $1 \mathrm{~h}$ after dosing. Cobalt concentration was analysed using atomic absorption spectrophotometry, and D-mannitol concentration was measured using a commercially available colorimetric assay kit (ab 155890, Abcam, Cambridge, UK). Data were analysed by two-way analysis of variance using SPSS (IBM SPSS Statistics for Windows, v21.0. Armonk, NY) with main effect means shown (Table 1).

Results An interaction $(P=0.04)$ in plasma D-mannitol was observed immediately post mixing $(\mathrm{d} 14)$, with DEX-treated pigs not exposed to mixing $(216 \mathrm{nmol} / \mathrm{L})$ having lower plasma $\mathrm{D}$-mannitol compared to saline-dosed pigs not exposed to mixing (485 nmol/L) and DEX-treated pigs exposed to mixing (496 nmol/L) but not saline dosed mixed pigs (398nmol/L). D-mannitol concentration was lower $(P=0.024)$ in pigs dosed with DEX compared to control pigs two days post mixing (d 16). No changes were seen in the concentration of Cobalt at d 14 or 16. These results suggest DEX supplementation reduced intestinal permeability, possibly due to its anti-inflammatory properties. In this regard, weaner pigs given $0.2 \mathrm{mg}$ $\mathrm{DEX} / \mathrm{kg}$ body weight $1 \mathrm{~d}$ prior to weaning and $3 \mathrm{~d}$ after weaning had significantly lower TNF- $\alpha$ than saline dosed pigs (Wooten et al., 2019). Higher levels of TNF- $\alpha$ in plasma indicate increased intestinal permeability.

Table 1. Paracellular permeability in weaner pigs given saline or DEX with/without mixing at d 14 after weaning.

\begin{tabular}{lcccccccc}
\hline & \multicolumn{2}{c}{ Treatment } & \multicolumn{2}{c}{ Stressor } & \multicolumn{3}{c}{$P$ value } \\
& Control & DEX & Mixing & Non-mixing & SEM & Treatment & Stressor & T x S \\
\hline D-Mannitol d 14 (nmol/L) & 441 & 356 & 446 & 351 & 41.1 & 0.317 & 0.261 & 0.041 \\
D-Mannitol d 16 (nmol/L) & 456 & 230 & 387 & 298 & 44.8 & 0.024 & 0.340 & 0.145 \\
\hline
\end{tabular}

Conclusion Dexamethasone supplementation in weaner pigs has the ability to minimise GIT dysfunction after acute mixing stress by minimising paracellular permeability. However, further research should be conducted to determine the association between the timing and form of DEX administration and exposure to the acute stressor.

Acknowledgements Supported in part by Australian Pork Limited.

\section{References}

Mereu A, Tedó G, Moeser AJ, Rimbach G and Ipharraguerre IR 2015. BMC Veterinary Research. 11, 274.

Turpin DL, Langendijk P, Chen T-Y and Pluske JR 2016. Animals. 6, 66.

Udén P, Colucci PE and Van Soest PJ 1980. Journal of the Science of Food and Agriculture. 31, 625-632.

Wooten H, McGlone JJ, Wachtel M, Thompson G, Rakhshandeh AR and Rakhshandeh A 2019. Animal. 10, 1-10. 


\section{Effect of imprinting with essential oils on performance and health parameters in weaned piglets}

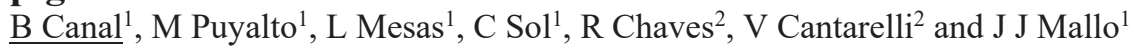

${ }^{l}$ NOREL SA, Spain, ${ }^{2}$ Universidade Federal de Lavras, Brasil

bcanal@norel.net

Application Post weaning diarrhoea can be reduced through the imprinting effect of essential oils.

Introduction Weaning is a key period for piglets, with weight loss occurring as the animal experiences stressful situations such as transition from liquid to solid diets, change in temperature or separation of the mother (Campbell et al., 2013). Many strategies have been studied to reduce this stress and to improve their immunological status which ultimately could translate to better health and performance. The exposure and conditioning of piglets to certain flavours based on essential oils (EO) through the amniotic fluid (during gestation) and the milk (during lactation) is one of the strategies producers are considering. The goal is to increase the acceptability and intake of feeds during the weaning. Apart from flavouring properties essential oils are also known for their antimicrobial activity (Chouhan et al., 2017), which could improve the animal's health parameters. The hypothesis is that essential oils can enhance both production and health parameters through the imprinting process using essential oils in sow and piglet diets.

Material and methods The trial used a 2 × 3 factorial design, with 2 sow diets and 3 piglet diets. A total of 180 sows received 2 different diets $(\mathrm{n}=90)$ during lactation: basal (B) or basal with EO (Print-Arome ${ }^{\circledR}$, Norel SA, Madrid, Spain) based on oregano and cinnamon essential oils) at $375 \mathrm{~g} / \mathrm{t}(\mathrm{T})$. During the nursery phase, 3 piglet diets were fed: basal diet (B), basal medicated with $200 \mathrm{ppm}$ of Colistin (A) or basal with $375 \mathrm{~g} / \mathrm{t}$ of the same EO (EO). A total of 168 piglets ( $\mathrm{n}=7$ ), weaned at d 25 were randomly distributed in the 6 groups: 3 from basal sows (BB, BA and BEO) and 3 from EO sows (TB, TA and TEO), the TEO group being those animals imprinted. Performance parameters were recorded at $14 \mathrm{~d}$ postweaning including body weight (BW), average daily gain (ADG), average daily feed intake (ADFI) and feed conversion ratio (FCR). Incidence of diarrhoea was recorded daily and calculated using the $\%$ of positive pens for diarrhoea (presence or absence) over the total period of observations carried out. Forty-two piglets were sacrificed (1 per pen) on d 9 postweaning and samples of duodenum, jejunum and ileum collected to perform histological evaluation. Caecal content was collected also for microbiota evaluation, quantifying Bifidobacteria, Lactobacillus and E. coli. Finally, blood samples were collected on d 1 post-weaning to evaluate the health status. Data were analysed by two-way ANOVA using GLM procedure of SAS (SAS Institute Inc., Cary, NC, USA).

Results There were no significant differences in performance parameters. However, the incidence of diarrhoea in the TEO group was $4.7 \%$, being significantly lower $(P=0.001)$ than other treatments. No differences were shown by the histological study between treatments, as well as the microbiota which was not significantly affected by the treatments. The levels of haptoglobin tended to be lower $(P=0.066)$ in the imprinted animals from the TEO group compared with the same group of sows TB and TA and no significant differences with the animals from the other group of sows.

Table 1. Effect of the addition of essential oils to sow (T) and piglet diets, basal (B), antibiotic (A) and essential oils (EO) on performance; ADG, average daily gain; ADFI, average daily feed intake; FCR, feed conversion ratio, and health parameters in weaned piglets.

\begin{tabular}{|c|c|c|c|c|c|c|c|c|c|c|}
\hline & \multicolumn{3}{|c|}{ Sow basal diet } & \multicolumn{3}{|c|}{ Sow basal diet + EO } & \multirow[b]{2}{*}{ SEM } & \multicolumn{3}{|c|}{$P$ value } \\
\hline & $\mathrm{BB}$ & $\mathrm{BA}$ & $\mathrm{BEO}$ & TB & TA & TEO & & SD & PD & SDxPD \\
\hline Initial weight (kg) & 5.94 & 5.95 & 5.95 & 5.99 & 5.98 & 5.98 & 0.160 & 0.800 & 1.000 & 1.000 \\
\hline Final weight (kg) & 8.12 & 7.94 & 8.18 & 8.03 & 8.00 & 8.24 & 0.219 & 0.970 & 0.580 & 0.940 \\
\hline $\mathrm{ADG}(\mathrm{g})$ & 156 & 141 & 157 & 156 & 144 & 160 & 6.149 & 0.940 & 0.330 & 0.990 \\
\hline ADFI (g) & 178 & 187 & 185 & 184 & 210 & 196 & 8.508 & 0.230 & 0.440 & 0.810 \\
\hline FCR $(g / g)$ & 1.23 & 1.33 & 1.27 & 1.22 & 1.23 & 1.14 & 0.047 & 0.130 & 0.450 & 0.660 \\
\hline Diarrhoea $(\%)$ & $10.8^{\mathrm{a}}$ & $13.7^{\mathrm{a}}$ & $10.7^{\mathrm{a}}$ & $14.4^{\mathrm{a}}$ & $12.9^{\mathrm{a}}$ & $4.7^{\mathrm{b}}$ & - & 0.367 & 0.001 & $<0.001$ \\
\hline Haptoglobin (ng/mL) & $97.2^{x y}$ & $96.0^{x y}$ & $100.6^{x y}$ & $107.6^{x}$ & $105.3^{x}$ & $91.3^{y}$ & 0.875 & 0.362 & 0.334 & 0.066 \\
\hline
\end{tabular}

${ }^{\mathrm{a}, \mathrm{b}}$ Means within a row with different superscripts differ significantly $(P<0.05)$; ${ }^{\mathrm{x}, \mathrm{y}}$ Means within a row with different superscripts differ significantly $(P<0.10)$; SD, sow diet; PD, piglet diet; SDxPD, interaction between diets.

Conclusion It can be concluded that in this trial the essential oils were not able to significantly improve performance parameters of the piglets during the first two weeks post weaning, although the conditioning with essential oils reduced significantly the incidence of diarrhoea. Histological and microbiota studies showed that there were no significant differences in intestinal tissue or bacteria population. A trend of lower levels of haptoglobin related to an interaction between sow and piglet diets was observed in the group of imprinted animals (TEO). Further studies are required to elucidate the mechanism of action behind the results obtained in this trial.

\section{References}

Campbell JM, Crenshaw JD and Polo J 2013. Journal of Animal Science and Biotechnology, 4, 19.

Chouhan S, Sharma K and Guleria S 2017. Medicines (Basel), 4, E58. 


\title{
Efficacy of medium chain fatty acid based additive at graded dose levels on performance and faecal consistency in piglets
}

$\mathrm{C} \mathrm{Sol}^{1}, \mathrm{M} \mathrm{Puyalto}^{1}, \mathrm{~J} \mathrm{~J}$ Mallo ${ }^{1}$ and J Zentek ${ }^{2}$

${ }^{1}$ NOREL SA, Spain, ${ }^{2}$ Freie Universität Berlin, Germany

csol@norel.net

\begin{abstract}
Application The application of the present trial is to establish the optimal dose of a medium chain fatty acid based additive according to the post-weaned piglets' performance.
\end{abstract}

Introduction Weaning is a stressful period due to changes in diet composition, environment and bacterial challenges. To date, not a single strategy has proved to be totally effective and it is necessary to develop strategies that involve a combination of diet modification with other preventive measures at farm level. The new feed additive based in sodium salts of medium chain fatty acids (Dicosan ${ }^{\circledR}$, NOREL SA, Madrid, Spain) contains high levels of lauric acid effective against the overgrowth of pathogenic bacteria in the gut of post-weaning piglets. The aim of this study was to investigate the optimal dosage of this additive by using graded dose levels supplemented into diets for post-weaning piglets from $\mathrm{d}$ 25 to d 66 of age (42d feeding period), with the hypothesis that performance was dose dependent. This study was performed following the current European Food Safety Authority (EFSA) guidance for tolerance and efficacy studies (EFSA, 2011) by targeting growth performance.

Material and methods Six treatments with increasing amounts of the feed additive $(0,2,4,6,8,10 \mathrm{~g} / \mathrm{kg}$ feed) supplemented into isoenergetic and isonitrogenous diets were offered to post-weaning piglets from d 25 to $\mathrm{d} 66$ of age (42 d). A starter diet was fed from d 1 to 14 and a grower diet from d 15 to d 42. The basal ingredients for the diets were corn, wheat, soybean meal, barley and skim milk powder for starter and wheat, corn, soybean meal, barley for grower diets, with the additive added to the basal diets at the expense of soybean oil and Tixosil (silicon dioxide). A total of 360 post-weaning barrows and gilts (DanBred x Duroc) were randomly allocated into 60 pens with 6 pigs per pen $(n=10)$. Pen body weights (BW) were recorded weekly, and the feed supplied to each pen over each preceding week. Feed conversion ratio (FCR) was calculated from the relationship of weekly corrected feed intake (FI) and growth per piglet for this period. Faecal consistency (on a pen basis) was ranked and averaged weekly (1=liquid diarrhoea; $2=$ pasty faeces falling out of shape upon contact with surfaces; $3=$ formed faeces, soft to cut; $4=$ well-formed faeces, firm to cut; $5=$ hard and dry faeces). All measurements were taken at pen-level and were analysed by one-way ANOVA with treatment as explanatory variable, adjusted by Tukey test (IBM SPSS Statistics for Windows, v25.0. Armonk, NY).

Results The initial body weight of the selected post-weaning piglets reached on average $7.43 \mathrm{~kg}$ and was similar across treatment groups. Piglets receiving $8 \mathrm{~g} / \mathrm{kg}$ of the additive showed significantly higher body weight gain (BWg) compared to 0 or $2 \mathrm{~g} / \mathrm{kg}$ (Table 1). Increasing the dosage to $10 \mathrm{~g} / \mathrm{kg}$ resulted in a non-significant decrease in efficiency compared to $8 \mathrm{~g} / \mathrm{kg}(-6 \%)$. Similarly dose dependent effects were observed during the starter and grower period. There was no treatment effect on FI. FCR was significantly reduced by an average of $8.9 \%$ when the additive was included at 6 to 10 $\mathrm{g} / \mathrm{kg}$. Regarding the overall feeding period faecal scores observed in piglets $8 \mathrm{~g} / \mathrm{kg}$ were significantly better than those presented in the $0 \mathrm{~g} / \mathrm{kg}(3.70$ vs 3.90) and the $10 \mathrm{~g} / \mathrm{kg}(3.79$ vs 3.90).

Table 1. Effect of increasing dose levels of a feed additive containing sodium salts of medium chain fatty acids on growth performance and faecal consistency in piglets from d 25 to d 66 of age

\begin{tabular}{ccccc}
\hline Treatment & BWg $(\mathrm{kg})$ & FI $(\mathrm{kg})$ & FCR $(\mathrm{kg} / \mathrm{kg})$ & Faecal score \\
\hline $0 \mathrm{~g} / \mathrm{kg}$ & $15.92^{\mathrm{a}} \pm 1.25$ & $24.86 \pm 1.87$ & $1.56^{\mathrm{c}} \pm 0.045$ & $3.70^{\mathrm{a}} \pm 0.14$ \\
$2 \mathrm{~g} / \mathrm{kg}$ & $16.35^{\mathrm{a}} \pm 1.36$ & $24.74 \pm 1.70$ & $1.51^{\mathrm{bc}} \pm 0.034$ & $3.82^{\mathrm{abc}} \pm 0.13$ \\
$4 \mathrm{~g} / \mathrm{kg}$ & $16.63^{\mathrm{ab}} \pm 1.33$ & $24.56 \pm 1.78$ & $1.48^{\mathrm{bc}} \pm 0.053$ & $3.82^{\mathrm{abc}} \pm 0.10$ \\
$6 \mathrm{~g} / \mathrm{kg}$ & $17.26^{\mathrm{ab}} \pm 1.05$ & $24.77 \pm 1.56$ & $1.44^{\mathrm{ab}} \pm 0.057$ & $3.90^{\mathrm{bc}} \pm 0.08$ \\
$8 \mathrm{~g} / \mathrm{kg}$ & $18.04^{\mathrm{b}} \pm 1.19$ & $25.09 \pm 2.39$ & $1.39^{\mathrm{a}} \pm 0.058$ & $3.90^{\mathrm{c}} \pm 0.07$ \\
$10 \mathrm{~g} / \mathrm{kg}$ & $16.73^{\mathrm{ab}} \pm 0.90$ & $24.16 \pm 1.24$ & $1.45^{\mathrm{ab}} \pm 0.035$ & $3.79^{\mathrm{ab}} \pm 0.24$ \\
$P$ value & 0.004 & 0.935 & $<0.001$ & 0.001 \\
\hline
\end{tabular}

${ }^{\text {a,b,c }}$ Means within a column with different superscripts differ significantly $(P<0.05)$; BWg, body weight gain across the study period; FI, feed intake across the study period; FCR, feed conversion ratio

Conclusion The additive had dose dependent positive effects on growth performance up to a dosage of $8 \mathrm{~g} / \mathrm{kg}$. Based on the significant quadratic regressions between inclusion amount and $\mathrm{BWg}\left(\mathrm{y}=15.726+0.438 \mathrm{x}-0.030 \mathrm{x}^{2} ; P=0.003\right)$ or FCR $\left(y=1.571-0.036 \mathrm{x}+0.002 \mathrm{x}^{2} ; P=0.003\right)$, the optimal dosage of Dicosan ${ }^{\circledR}$ for piglets from $\mathrm{d} 25$ to $\mathrm{d} 66$ of age was calculated to be between 6 and $8 \mathrm{~g} / \mathrm{kg}$ feed.

Acknowledgements Supported in part by the European Union in the Operative Program "FEDER" for the project LIPOXIFEED (COMRDI16-1-0033).

\section{References}

EFSA 2011. EFSA Journal. 9, 2175. 


\section{Effects of dietary supplementation of non-starch polysaccharide enzymes on the growth performance and nutrient digestibility of grower-finisher pigs}

$\mathrm{Y} \mathrm{Luo}^{1}$, $\mathrm{H}_{\text {Zhai }}{ }^{1}$, S Wang ${ }^{1}$ and $\mathrm{J} \mathrm{Wu}^{1}$

${ }^{I}$ DSM (China) Animal Nutrition Research Center Co., Ltd, China

heng-xiao.zhai@dsm.com

Application Supplementation of non-starch polysaccharide enzymes (NSPase) in a barley, wheat, rapeseed and sunflower meal diet could improve the growth rate and body weight of pigs during finisher phase.

Introduction Non-starch polysaccharides (NSPs), such as cellulose, arabinoxylan, glucan and pectin, decrease the digestion of protein, fat, and some minerals, which might cause a lower energy supply to the pig and a shortage in protein and some minerals (Bakker et al., 1998). One percentage point increase of NSP in the diet can result in a depression of precaecal digestibility of organic matter by 1.5-2.0\% (Drochner et al., 2004). Cereals, such as barley and wheat are rich in $\beta$-glucan and arabinoxylan, while oilseed meals such as rapeseed and sunflower meal are more abundant in pectin and cellulose. NSPase (RONOZYME® VP, DSM Nutritional Products, China) contains $\beta$-glucanase, hemicellulase, and pectinase, which can hydrolyse NSPs and thereby increase nutrient digestibility and improve the growth performance of pigs. Therefore, it was hypothesised that the supplementation of NSPase in a barley, wheat, rapeseed and sunflower meal diet would result in an improvement in nutrient digestibility and growth performance of pigs.

Material and methods A growth performance and metabolic trial was conducted to investigate the efficacy of the NSPase. There were three dietary treatments in the trial including a negative control (NC) and the NC supplemented with NSPase at 0.1 and $0.2 \mathrm{~g} / \mathrm{kg}$ feed. Two hundred and eighty-eight PIC 1050 x 337 barrows and gilts (initial body weight $28.8 \pm 2.8 \mathrm{~kg}$ ) were used with 16 replicate pens for each treatment and 6 pigs (3 barrows and 3 gilts) in each replicate pen. The trial lasted for 10 weeks divided into a grower and a finisher phase, 5 weeks each phase. Twenty-four barrows (initial body weight $68.6 \pm 6.2 \mathrm{~kg}$ ) were used in the metabolic trial. This trial lasted for $10 \mathrm{~d}$, consisted of a $5 \mathrm{~d}$ adjustment period and a $5 \mathrm{~d}$ period of total collection of faeces. Titanium dioxide was used as the indicator to determine the total tract digestibility of dry matter, gross energy, crude protein, crude fat, crude fibre, calcium and phosphorus.

Results The results showed that with the increasing supplementation of NSPase, average daily gain during week 5 to 10 and 0 to 10, and average body weight on d 70 improved linearly $(P<0.05)$. The supplementation of NSPase at 0.1 and $0.2 \mathrm{~g} / \mathrm{kg}$ feed did not significantly influence the growth performance of pigs during week 0 to 5 (not shown) and the digestibility of dry matter, gross energy, crude protein, crude fat, crude fibre, or phosphorus, but the digestibility of calcium improved linearly $(P<0.05)$ with increasing supplementation of NSPase.

Table 1. Growth performance; ADG, average daily gain; ADFI, average daily feed intake; FCR, feed conversion ratio, of grower-finisher pigs, and apparent total tract digestibility (ATTD) in finisher pigs.

\begin{tabular}{|c|c|c|c|c|c|c|}
\hline & \multicolumn{3}{|c|}{ NSPase inclusion rate } & \multirow[b]{2}{*}{ SEM } & \multicolumn{2}{|c|}{$P$ value } \\
\hline & $0.0 \mathrm{~g} / \mathrm{kg}$ & $0.1 \mathrm{~g} / \mathrm{kg}$ & $0.2 \mathrm{~g} / \mathrm{kg}$ & & Linear & Quadratic \\
\hline d 0 weight (kg) & 28.8 & 28.8 & 28.8 & 0.06 & 0.93 & 0.68 \\
\hline d 35 weight $(\mathrm{kg})$ & 64.0 & 64.1 & 64.3 & 0.34 & 0.54 & 0.90 \\
\hline d 70 weight $(\mathrm{kg})$ & $102.2^{\mathrm{a}}$ & $103.5^{\mathrm{ab}}$ & $104.1^{\mathrm{b}}$ & 0.50 & 0.02 & 0.63 \\
\hline w5-10 ADG (g/d) & $1093^{a}$ & $1125^{\mathrm{ab}}$ & $1137^{\mathrm{b}}$ & 10 & $<0.01$ & 0.42 \\
\hline w5-10 ADFI (g/d) & 3181 & 3205 & 3244 & 36 & 0.23 & 0.87 \\
\hline w5-10 FCR (g/g) & 2.91 & 2.85 & 2.85 & 0.03 & 0.15 & 0.34 \\
\hline w0-10 ADG (g/d) & 1049 & 1063 & 1073 & 8 & 0.03 & 0.86 \\
\hline w0-10 ADFI (g/d) & 2762 & 2763 & 2799 & 29 & 0.37 & 0.63 \\
\hline w0-10 FCR $(\mathrm{g} / \mathrm{g})$ & 2.63 & 2.60 & 2.61 & 0.02 & 0.28 & 0.34 \\
\hline ATTD Dry matter (\%) & 77.02 & 76.94 & 76.72 & 0.13 & 0.61 & 0.88 \\
\hline ATTD Crude protein $(\%)$ & 76.85 & 76.02 & 77.03 & 0.30 & 0.89 & 0.44 \\
\hline ATTD Crude fibre (\%) & 14.83 & 16.17 & 15.39 & 0.46 & 0.79 & 0.56 \\
\hline ATTD Crude fat $(\%)$ & 85.21 & 86.10 & 85.82 & 0.79 & 0.86 & 0.85 \\
\hline ATTD Calcium (\%) & $46.93^{\mathrm{a}}$ & $49.67^{\mathrm{ab}}$ & $51.19^{\mathrm{b}}$ & 0.37 & 0.02 & 0.68 \\
\hline ATTD Phosphorus (\%) & 35.99 & 37.37 & 36.91 & 0.38 & 0.59 & 0.54 \\
\hline ATTD Gross Energy (\%) & 77.21 & 77.26 & 77.23 & 0.15 & 0.98 & 0.95 \\
\hline
\end{tabular}

${ }^{\mathrm{a}, \mathrm{b}}$ Means within a row with different superscripts differ significantly $(P<0.05)$; SEM, standard error of the means

Conclusion The supplementation of NSPase improved growth rate and final average body weight, and the digestibility of calcium of pigs during finisher phase. Diet based on barley and wheat supply diverse quantities of specific substrates especially $\beta$-glucan for microbial fermentation, which may affect microbial activity and metabolite production (Leek et $a l .$, 2007). Therefore, more in-depth analysis such as microbiome shifts are required to explain the mechanism.

\section{References}

Bakker GCM, Dekker RA, Jongbloed R and Jongbloed AW 1998. Veterinary Quarterly. 20, 59-64.

Drochner W, Kerler A and Zacharias B 2004. Journal of Animal Physiology and Animal Nutrition. 88, 367-380.

Leek ABG, Callan JJ, Reilly R, Beattie VE and O’Doherty JV 2007. Animal Feed Science and Technology. 135, 86-99. 


\section{Dietary caffeine reduces feed intake and improves feed efficiency in female grower pigs}

C J Braden $^{1}$, R S Morrison ${ }^{1}$, F Liu $^{1}$ and C J Brewster ${ }^{1}$

${ }^{1}$ Rivalea Australia Pty Ltd, Australia

cbraden@rivalea.com.au

Application Including caffeine in grower diets significantly reduced average daily feed intake and feed conversion ratio but had no effect on ultrasound backfat thickness (at P2 site).

Introduction Caffeine is a bitter compound that reduces feed intake and backfat thickness in finisher pigs (Fu et al., 2015). However, there is limited research into the effects of caffeine inclusion in lighter pigs, where energy intakes are lower. It is hypothesised that caffeine inclusion in the diets of younger pigs may further limit energy intake and be a useful tool in manipulating feed intake and fat deposition in pigs. The aim of this experiment was to investigate the effect of dietary caffeine inclusion on the growth performance and body composition of pigs between 25 and $55 \mathrm{~kg}$ live weight.

Material and methods The experiment was conducted using 40 female grower $(23.8 \pm 0.4 \mathrm{~kg})$ pigs (Large White $\mathrm{x}$ Landrace $\mathrm{x}$ Duroc, Primegro ${ }^{\mathrm{TM}}$ Genetics, Corowa, NSW). The pigs were housed in individual pens and randomly allocated to two dietary treatments. Diets contained $14 \mathrm{MJ}$ of digestible energy $/ \mathrm{kg}(\mathrm{MJ} \mathrm{DE} / \mathrm{kg}$ ) and $0.7 \mathrm{~g}$ of standard ileal digestible lysine/MJ DE (SID lys/MJ) and two caffeine levels (0 and $500 \mathrm{~g} / \mathrm{t})$. The pigs were individually weighed and backfat was recorded via ultrasound (CTS-900, SIUI, Shantou, China) at the P2 site, $65 \mathrm{~mm}$ from the midline at the head of the last rib, at 9 (d 0) and 14 weeks (d 35) of age. Individual daily feed intakes were recorded. Data were analysed using UNIVARIATE procedure for the effects of dietary treatment (IBM SPSS Statistics for Windows, v24.0. Armonk, NY) using the individual pig as the experimental unit, with weight and backfat thickness at $\mathrm{d} 0$ as covariate in respective analyses.

Results Average daily feed intake (ADFI) and feed conversion ratio (FCR) were significantly lower in the caffeine treatment $(P<0.05$, Table 1$)$. There was no significant difference between treatments in average daily gain (ADG), weight (d 35) or backfat thickness (d 35).

Table 1. Weight, backfat thickness at the P2 site, average daily feed intake (ADFI), average daily gain (ADG) and feed conversion (FCR) of female grower pigs offered diets without (Control) or with (Caffeine) $500 \mathrm{~g} / \mathrm{t}$ caffeine.

\begin{tabular}{lccccc}
\hline & & Control & Caffeine & SEM & $P$ value \\
\hline Weight d 0 & $(\mathrm{kg})$ & 23.9 & 23.8 & 0.36 & 0.87 \\
Weight d 35 & $(\mathrm{kg})$ & 52.0 & 51.3 & 0.90 & 0.62 \\
Backfat thickness d 0 & $(\mathrm{mm})$ & 6.7 & 6.8 & 0.11 & 0.85 \\
Backfat thickness d 35 & $(\mathrm{mm})$ & 9.9 & 9.3 & 0.25 & 0.19 \\
ADFI & $(\mathrm{kg} / \mathrm{d})$ & $1.63^{\mathrm{a}}$ & $1.43^{\mathrm{b}}$ & 0.04 & 0.014 \\
ADG & $(\mathrm{kg} / \mathrm{d})$ & 0.81 & 0.78 & 0.02 & 0.59 \\
FCR & $(\mathrm{kg} / \mathrm{kg})$ & $2.03^{\mathrm{a}}$ & $1.84^{\mathrm{b}}$ & 0.03 & 0.003 \\
\hline
\end{tabular}

a,b Means within a row with different superscripts differ significantly $(P<0.05)$; SEM, standard error of the mean

Conclusion Inclusion of caffeine in the diet of grower pigs reduced feed intake and feed conversion without affecting growth rate but did not significantly reduce backfat thickness measured by ultrasound on live pigs. Further research is required to investigate longer duration of caffeine use in grower and finisher diets, as well as the ability of caffeine to improve feed conversion without affecting the growth rates of younger pigs.

Acknowledgements Staff at the Research and Innovation Unit and farming operations at Rivalea Australia Pty Ltd are thanked for their support.

\section{References}

Fu M, Collins CL, Henman DJ and Roura E 2015. Animal Production Science. 55, 1543. 


\section{Feeding the same diet to pigs in the grower-finisher period reduces feed costs compared to phase feeding with no effect on growth performance and carcass quality}

$\underline{K}^{2}$ L Moore ${ }^{1}$, T Clarke ${ }^{2}$ and A C Edwards ${ }^{3}$

${ }^{1}$ Pork Innovation WA Inc., Australia, ${ }^{2}$ Westpork Pty Ltd, Australia, ${ }^{3}$ ACE Livestock Consulting, Australia

karen@klmconsulting.com.au

Application Feeding the same diet in the grower-finisher period reduces feed costs with no effect on growth performance compared to phase feeding. Feeding a single diet to pigs in the grower finisher stage has several advantages for feed manufacture, storage and delivery.

Introduction Feeding the same diet through the grower-finisher period (single) is a possible alternative to the widely used phase feeding strategy where approximately three to four diets are fed. Moore et al. $(2012,2016)$ found that feeding a single diet targeted to meet lysine requirements at $60 \mathrm{~kg}$ live weight (LW) reduced digestible energy intake per $\mathrm{kg} \mathrm{LW}$ gain and hence reduced feed costs by between 3.5 to $5 \%$ compared to a three-phase feeding regime with no impact on overall growth performance or carcass quality. Given the research of Moore et al. $(2012,2016)$ was conducted in a research facility with low variation there were questions from industry over the validity in a commercial facility. The hypotheses were that (1) feeding single diet will reduce the cost of feeding pigs compared to the phase system without adversely affecting pig growth performance and carcass quality and (2) feeding a single diet targeted to meet lysine requirements at $70 \mathrm{~kg}$ liveweight will reduce the cost of feeding compared to feeding a single diet targeted to meet lysine requirements at either 50 or $60 \mathrm{~kg}$ liveweight.

Material and methods A completely randomised block experiment was conducted in a commercial facility using 1120 female pigs (Large White x Landrace x Duroc, 40 pigs/pen and seven replicate pens/treatment) to examine the effect of feeding strategies on performance during the grower-finisher phase. Pigs were randomly allocated to the following feeding strategies: (1) Phase: diets changed when the average LW of pigs reached $23 \mathrm{~kg}$ (14.4 MJ digestible energy (DE) $/ \mathrm{kg}, 0.84 \mathrm{~g}$ standardised ileal digestible lysine (SID Lys)/MJ DE and $0.30 \%$ available phosphorus (Av Phos)), 50 (13.8 MJ DE/kg, $0.67 \mathrm{~g}$ SID Lys/MJ DE and 0.311\% Av Phos), 70 (13.6 MJ DE/kg, $0.60 \mathrm{~g}$ SID Lys/MJ DE and 0.317\% Av Phos) and $90 \mathrm{~kg}$ (13.3 MJ DE/kg, $0.52 \mathrm{~g}$ SID Lys/MJ DE and 0.322\%); (2) Single 50: a single diet fed throughout that met the lysine requirements for $50 \mathrm{~kg} \mathrm{LW}(13.8 \mathrm{MJ}$ DE $/ \mathrm{kg}, 0.67 \mathrm{~g}$ SID Lys/MJ DE and $0.311 \%$ Av Phos); (3) Single 60: a single diet fed to meet lysine requirements at $60 \mathrm{~kg} \mathrm{LW}(13.7 \mathrm{MJ} \mathrm{DE} / \mathrm{kg}, 0.65 \mathrm{~g}$ SID Lys $/ \mathrm{MJ} \mathrm{DE}$ and $0.313 \% \mathrm{Av}$ Phos) and; (4) Single 70: a single diet fed to meet lysine requirements at $70 \mathrm{~kg} \mathrm{LW} \mathrm{(13.6} \mathrm{MJ} \mathrm{DE/} / \mathrm{kg}, 0.60 \mathrm{~g}$ SID Lys/MJ $\mathrm{DE}$ and $0.317 \%$ Av Phos). The experimental diets were fed from $23.3 \pm 0.80$ to $99.0 \pm 0.91 \mathrm{~kg} \mathrm{LW}$ (mean $\pm \mathrm{SE}$ ). All data were analysed by analysis of variance (GenStat 19th ed, VSN International, Hemel Hempstead, UK).

Results There was no significant effect of feeding strategy on growth performance, carcass weight or back fat $(P>0.05$; Table 1). There was a trend for pigs who received the Single 60 or Single 70 diet to have an improved feed conversion ratio compared to those fed the Phase or Single 50 diet $(P=0.062)$. It was cheaper to feed pigs the Single 70 diet, than the Single 60 diet, which in turn was cheaper than both the Single 50 and Phase diets $(P<0.001)$.

Table 1. Growth performance, carcass quality and feed costs for female pigs fed four different feeding strategies $(\mathrm{n}=7)$.

\begin{tabular}{|c|c|c|c|c|c|c|}
\hline & Phase & Single 50 & Single 60 & Single 70 & $\mathrm{SED}^{1}$ & $P$ value \\
\hline Initial weight $(\mathrm{kg})$ & 22.5 & 23.7 & 23.2 & 23.8 & 1.16 & 0.66 \\
\hline Daily gain $(\mathrm{kg} / \mathrm{d})^{2}$ & 0.766 & 0.772 & 0.771 & 0.767 & 0.017 & 0.98 \\
\hline Feed intake $(\mathrm{kg} / \mathrm{d})^{2}$ & 2.11 & 2.07 & 1.99 & 2.01 & 0.050 & 0.33 \\
\hline Feed conversion ratio & 2.76 & 2.69 & 2.57 & 2.63 & 0.066 & 0.06 \\
\hline Carcass weight $^{3}(\mathrm{~kg})$ & 72.1 & 72.5 & 72.2 & 72.4 & 0.420 & 0.63 \\
\hline Back fat $(\mathrm{mm})$ & 10.1 & 10.1 & 10.1 & 9.78 & 0.211 & 0.35 \\
\hline Feed costs $/ \mathrm{kg} \mathrm{LW}$ gain $(\$)^{4}$ & $1.80^{\mathrm{a}}$ & $1.82^{\mathrm{a}}$ & $1.66^{\mathrm{b}}$ & $1.48^{\mathrm{c}}$ & 0.040 & $<0.001$ \\
\hline
\end{tabular}

a,b,cc Means within a row with different superscripts differ significantly $(P<0.05) ;{ }^{1}$ SED, standard error of difference of the means; ${ }^{2}$ Initial liveweight used as a covariate; ${ }^{3}$ Carcass weight, hot carcass weight: AUSMEAT trim 13 - head off, flare off, fore trotters off, hind trotters on; LW, liveweight; ${ }^{4} \mathrm{Feed}$ costs, January 2019.

Conclusion To reduce diet costs in the grower finisher phase pigs can be fed the same diet (targeted to their lysine requirements at either 60 or $70 \mathrm{~kg} \mathrm{LW}$ ) with no effect on growth performance or carcass quality. Compared to phase feeding it was cheaper to feed the Single 60 and Single 70 diet by 0.14 and $0.32 \mathrm{c} / \mathrm{kg}$ LW gain, respectively. In addition to reducing the diet cost, feeding a single diet to pigs in the grower finisher phase only requires one diet which may simplify the manufacture, storage and delivery process.

Acknowledgements Supported in part by Australasian Pork Research Institute Limited.

\section{References}

Moore KL, Kim JC and Mullan BP 2012. Animal Production Science. 53, 52-56.

Moore KL, Mullan BP and Kim JC 2016. Animal Feed Science and Technology. 216, 273-280. 


\section{Effect of a nutritional emulsifier in an energy reduced diet on the performance of fattening} pigs

K Meza ${ }^{1}$, L Rombola ${ }^{2}$, M Sinclair ${ }^{2}$, B Bruneel ${ }^{2}$ and L Segers ${ }^{2}$

${ }^{I}$ Montana SA, Peru, ${ }^{2}$ Orffa Additives BV, the Netherlands

Bruneel@orffa.com

Application Improving fat digestibility through the use of a nutritional emulsifier results in improved growth performance.

Introduction Energy is a major cost factor in swine diets. Due to their high energy density, fats and oils are important energy sources in feed formulation. Improving the energy efficiency of these raw materials is of much interest from an economical point of view. Nutritional emulsifiers can be used to improve fat digestibility and, thus, improve energy efficiency. This could result in more cost-effective diets without losing on performance. Previous studies on nutritional emulsifiers focused on the application of mixtures containing glycerol polyethylene glycol ricinolate (GPGR) (Sun and Kim, 2019), which showed that GPGR is an agent that can be used to improve emulsification of fats in swine. In this study, it is hypothesised that GPGR as such, can also have a significant impact on the performance of swine and in particular in energy reduced diets.

Material and methods Fifty-four male pigs ( $\mathrm{n}=54$ ), Yorkshire $\mathrm{x}$ Landrace, were allocated to two groups: i) control group (CG), where pigs had continuous access to a corn-soy based diet (13.8 MJ DE $/ \mathrm{kg}, 1.15 \%$ standard ileal digestible lysine) with $1.77 \%$ soybean oil and ii) emulsifier group (EG), where pigs had continuous access to a reduced energy (13.6 MJ $\mathrm{DE} / \mathrm{kg}$ ) corn-soy based diet with the same crude protein level (17\%) and amino acid level (1.15\% standard ileal digestible lysine and $0.39 \%$ digestible methionine supplemented with a GPGR based nutritional emulsifier with a specific high hydrophilic-lipophilic balance (HLB), Excential Energy Plus $(0.035 \%$, Orffa Additives BV, Werkendam, the Netherlands). At the start of the trial, pigs ( $70 \mathrm{~d}$ of age, $29.5 \pm 2.04 \mathrm{~kg}$ ) were divided over 12 experimental units (6 CG and $6 \mathrm{EG}$ ) each containing a maximum of five animals. After a $29 \mathrm{~d}$ trial period the following parameters were analysed: body weight (BW, $\mathrm{kg}$ ), body weight gain (BWG, $\mathrm{kg}$ /day), feed intake (FI, $\mathrm{kg}$ /day), feed conversion ratio (FCR) and dorsal fat thickness (DFT, mm). Data were analysed by usage of the ANOVA procedure and the comparison of means using the LSD test $(P<0.05$, SAS Institute Inc., Cary, NC, USA).

Results BW and BWG were significantly higher $(P<0.05$, Table 1) for EG pigs compared to CG pigs. The inclusion of a nutritional emulsifier did not impact other parameters.

Table 1. Growth performance, body weight (BW), body weight gain (BWG), feed intake (FI), feed conversion ratio (FCR) and dorsal fat thickness (DFT) of male fattening pigs, starting weight $29.5 \pm 2.04 \mathrm{~kg}$, fed a control diet (CG) or a diet containing a nutritional emulsifier (EG).

\begin{tabular}{lccc}
\hline & CG & EG & $P$ value \\
\hline BW $(\mathrm{kg})$ & $51.4^{\mathrm{a}}$ & $52.2^{\mathrm{b}}$ & 0.046 \\
BWG $(\mathrm{kg} / \mathrm{d})$ & $0.761^{\mathrm{b}}$ & $0.796^{\mathrm{a}}$ & 0.038 \\
FI $(\mathrm{kg} / \mathrm{d})$ & 1.60 & 1.64 & 0.326 \\
FCR $(\mathrm{kg} / \mathrm{kg})$ & 2.11 & 2.07 & 0.397 \\
DFT $(\mathrm{mm})$ & 7.5 & 7.7 & 0.781 \\
\hline
\end{tabular}

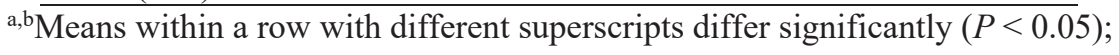

Conclusion These results suggest that a nutritional emulsifier, based on GPGR-technology only, is effective in energy reduced fattening pig diets, which supports our hypothesis.

\section{References}

Sun HY and Kim IH 2019 Livestock Science 227, 55-59. 


\section{Is it possible to reduce zinc supplementation in nursery piglets without affecting growth performance?}

M Rodríguez ${ }^{1}$, A Monteiro ${ }^{2}$, G Montalvo ${ }^{1}$, I Cockshott ${ }^{2}$, D Saunders ${ }^{3}$ and J Morales ${ }^{1}$

${ }^{1}$ PigCHAMP Pro Europa, Spain, ${ }^{2}$ ANIMINE, France, ${ }^{3}$ Link Asia Partners, Singapore

amonteiro@animine.eu

Application Using more bioavailable zinc ( $\mathrm{Zn}$ ) sources in piglet feed, it is possible to reduce the $\mathrm{Zn}$ supplementation in nursery piglets to $60 \mathrm{ppm}$ and keep the same performance as at high levels (for instance, 120 and $180 \mathrm{ppm}$ ).

Introduction The nutritional zinc requirement of weaned piglets (from 7 to $11 \mathrm{~kg}$ of body weight, BW) consuming a conventional diet has been established at $100 \mathrm{mg} / \mathrm{kg}$ of BW, as not all the zinc is available to the animal (NRC, 2012). If new sources of zinc were more bioavailable, there is the potential to further reduce the dietary zinc levels without affecting performance. The aim of this study (Project E! 11780 with funding from Eurostars-2) was to evaluate the efficacy of two sources of zinc oxide $(\mathrm{ZnO})$ : a potentiated $\mathrm{ZnO}\left(\mathrm{pZnO}\right.$, HiZox ${ }^{\circledR}$, Animine, France) and a conventional $\mathrm{ZnO}$, supplemented at different dosages ( $60 \mathrm{ppm}$ - to test lower $\mathrm{Zn}$ levels than the requirement, $120 \mathrm{ppm}$ - to benchmark with the EU Regulation, and $180 \mathrm{ppm}$ - to benchmark with countries where $\mathrm{Zn}$ level is not limited).

Material and methods At 28 days of age, 384 piglets (intact males and females) with an average BW of $8.80 \mathrm{~kg}$ were weaned and allotted to 48 pens ( 8 piglets/pen) according to BW and sex. The $35 \mathrm{~d}$ nursery phase was divided into $14 \mathrm{~d}$ of prestarter (11.44 MJ NE/kg; 1.4\% SID Lys) and $21 \mathrm{~d}$ of starter (10.50 MJ NE/kg; $1.2 \%$ SID Lys). In a $2 \times 3$ factorial block design, the effects of 2 sources of $\mathrm{ZnO}$ (pZnO vs. $\mathrm{ZnO}$ ) and 3 inclusion rates $(60,120$ and $180 \mathrm{ppm}$ ), on growth performance, mortality and diarrhoea scores were observed in the prestarter, starter and the whole nursery period. Growth performance was analyzed using the MIXED procedure and mortality and diarrhea were analysed using the FREQ procedure of SAS (SAS Institute Inc., Cary, NC, USA).

Results A source $\mathrm{x}$ dose effect $(P<0.05)$ was observed in average daily gain (from 28 to $63 \mathrm{~d}$ of age) and in final BW, which at the lowest dose supplementation (60 ppm) were higher in $\mathrm{pZnO}(0.55 \mathrm{~kg} / \mathrm{d}$ and $20.1 \mathrm{~kg}$, respectively) than in $\mathrm{ZnO}$ piglets $(0.50 \mathrm{~kg} / \mathrm{d}$ and $18.9 \mathrm{~kg}$, respectively). Average daily feed intake did not differ between treatments. At 60 $\mathrm{ppm}$, feed conversion ratio (FCR) did not differ in terms of zinc source, however, at $120 \mathrm{ppm} \mathrm{pZnO}$ presented lower FCR than $\mathrm{ZnO}$, whilst at $180 \mathrm{ppm} \mathrm{ZnO}$ presented lower FCR than $\mathrm{pZnO}(P<0.05$, Figure 1). Neither source nor dose affected mortality rate $(P>0.05$, Table 1$)$. Source did not affect presence of diarrhoea, but zinc supplemented at the highest dose $(180 \mathrm{ppm})$ decreased diarrhoea scores compared with lower doses $(P<0.05)$.

Average daily gain, $\mathrm{kg} / \mathrm{d}$

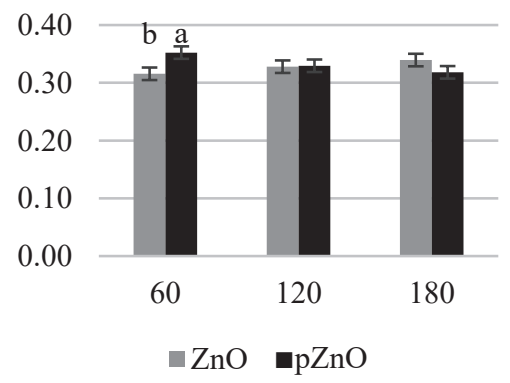

Average daily feed intake, $\mathrm{kg} / \mathrm{d}$

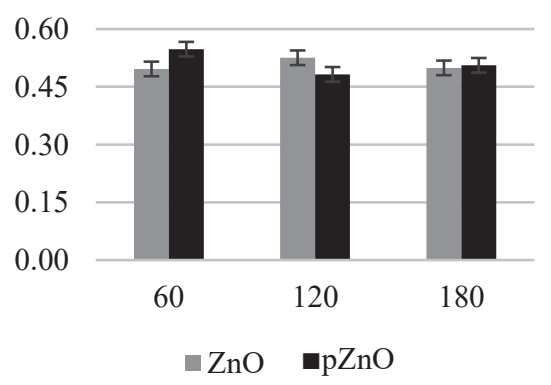

Feed conversion ratio, $\mathrm{kg} / \mathrm{kg}$

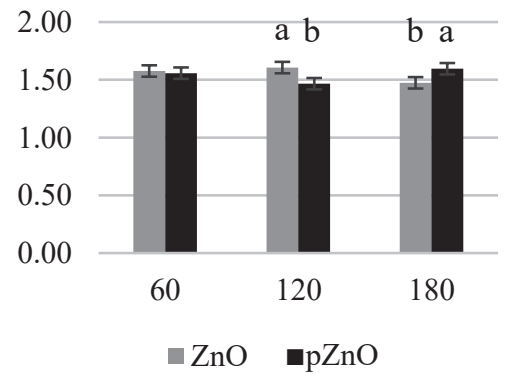

Figure 1. Performance of post-weaned pigs in prestarter and starter phase (d 28 to 63 of age), with zinc oxide ( $\mathrm{ZnO}$ ) or a potentiated zinc $(\mathrm{pZnO})$ both fed at 60,120 or $180 \mathrm{ppm}$.

Table 1. Mortality and presence of diarrhoea of post-weaned pigs in prestarter and starter phase (d 28 to 63 of age), with zinc oxide $(\mathrm{ZnO})$ or a potentiated zinc $(\mathrm{pZnO})$ both fed at 60,120 or $180 \mathrm{ppm}$.

\begin{tabular}{lcccccccc}
\hline Item & \multicolumn{2}{c}{ Source } & \multicolumn{3}{c}{ Dose } & \multicolumn{3}{c}{$P$ value } \\
& $\mathrm{ZnO}$ & $\mathrm{pZnO}$ & 60 & 120 & 180 & Source (S) & Dose (D) & $\mathrm{S} \times \mathrm{D}$ \\
\hline Mortality, \% & 2.08 & 3.65 & 2.34 & 3.13 & 3.13 & 0.36 & 0.91 & 0.74 \\
Diarrhoea, \% & 2.60 & 2.60 & 3.13 & 4.69 & 0.00 & 1.00 & 0.056 & 0.144 \\
\hline
\end{tabular}

Conclusion In conclusion, under the present field condition, by using more bioavailable sources of zinc, for instance a potentiated zinc oxide, dietary zinc can be reduced to $60 \mathrm{ppm}$ without affecting growth performance of weaning piglets.

Acknowledgements Supported in part by Eurostars-2 joint program (Project E! 11780) with co-financing from BPI (France), CDTI (Spain) and from the Horizon 2020 Research and Innovation Framework Program of the European Union.

\section{References}

NRC 2012. Nutrient Requirements of Swine: $11^{\text {th }}$ Revised Edition. The National Academies Press, Washington DC, USA. 
The inclusion of phytomolecules in weaner pig diets improves growth performance

$\underline{\text { R J E Hewitt }}{ }^{1}$, F F Barbosa ${ }^{2}$, J Grapentin ${ }^{2}$, D Sherwood ${ }^{2}$, S M Tritton ${ }^{1}$, A C Corso ${ }^{1}$ and D N D'Souza ${ }^{1}$

${ }^{1}$ SunPork Group, Australia ${ }^{2}$ EW Nutrition GmbH, Germany

robert.hewitt@sunporkfarms.com.au

Application Weaned pigs are subject to a range of stressors that can lead to reduced feed intake, impacting intestinal health and growth performance. The addition of phytomolecules to the diet of weaner pigs is shown to alleviate this postweaning growth check.

Introduction The separation of the weaner pig from its dam and litter mates, a change of environment, and the sudden change from a liquid to solid diet often results in a period of reduced feed intake. This can lead to marked structural and functional changes to the small intestine and contributes to an inflammatory response compromising barrier function (Pluske et al., 2018). Phytomolecules are used by the plant to communicate, to attract dispersal vectors or to defend itself from attack, rather than being involved in the primary growth or reproduction of the plant. These extracts have been shown to improve animal health through the enhancement of immune function, alteration of the gut microbiota and direct suppression of pathogens (Liu et al., 2018). We hypothesise that the inclusion of phytomolecules in the diet of weaner pigs will enhance post-weaning performance.

Material and methods Five-hundred and sixty male and female pigs (20 d of age, $5.56 \pm 0.15 \mathrm{~kg}, 50: 50$ male:female) entered the experiment over a four week period, were sorted by sex and size and assigned to pens ( $\mathrm{n}=14)$. Pigs within each pen were weighed and allocated to one of four treatments using a randomised block design, resulting in 10 replicates per treatment, with pen as the replicate. Isoenergetic and isonitrogenous first stage weaner diets $(14.9 \mathrm{MJ} \mathrm{DE} / \mathrm{kg}, 0.88 \mathrm{~g}$ standard ileal digestible lysine/MJ DE) were fed to all treatments for $28 \mathrm{~d}$, with diets only differing in the combination of potassium diformate (FORMI, ADDCON GmbH, Germany (KDF)), a blend of essential oils and organic acids (Porcinat+, JEFO Nutrition Inc, Canada (EO/OA)), and a blend of phytomolecules (Activo, EW Nutrition GmbH, Germany (PHM)). The treatments were i) Control - 0.4\% KDF, 0.1\% EO/OA and 0\% PHM; ii) PHM- 0.4\% KDF, 0.1\% EO/OA and 0.02\% PHM; iii) PHM minus EO/OA - 0.4\% KDF, $0 \%$ EO/OA and $0.02 \%$ PHM and; iv) PHM minus KDF - 0\% KDF, $0.1 \%$ EO/OA and $0.02 \%$ PHM. Performance data were analysed by ANOVA with treatment as a fixed factor, entry week as blocking factor and entry weight as a covariate (GenStat $19^{\text {th }}$ ed, VSN International, Hemel Hempstead, UK), with pairwise differences between treatments determined by LSD $(P<0.05)$. Removals were tested for significance via Chisquared analysis.

Results The addition of PHM to the diet of weaner pigs resulted in significant $(P<0.05)$ improvements in the efficiency of growth across the experimental period. Those treatments receiving PHM in the presence of KDF, significantly $(P<$ 0.05 ) improved growth performance when compared to the control, resulting in a 0.9 to $1.1 \mathrm{~kg}$ additional weight gain after 28 d. However, when KDF was removed from the treatment there was no significant difference in performance to the control. No difference in the number of removals was seen between treatments.

Table 1 Mean weight of pigs at weaning (d 1) and the end of the experimental period (d 28), average daily feed intake (ADFI), average daily gain (ADG), feed conversion ratio (FCR) and removals across the experimental period for pigs receiving the Control, the phytomolecule blend (0.02\% (PHM)), the PHM (0.02\%) minus blend of essential oils and organic acids $(0.1 \%(\mathrm{EO} / \mathrm{OA}))$, or the PHM $(0.02 \%)$ minus potassium diformate $(0.4 \%$ (KDF)) treatments.

\begin{tabular}{lcccccc}
\hline & $\begin{array}{c}\mathrm{d} 1 \text { weight } \\
(\mathrm{kg})\end{array}$ & $\begin{array}{c}\mathrm{d} 28 \text { weight } \\
(\mathrm{kg})\end{array}$ & $\begin{array}{c}\text { ADFI } \\
(\mathrm{kg} / \mathrm{d})\end{array}$ & $\begin{array}{c}\text { ADG } \\
(\mathrm{kg} / \mathrm{d})\end{array}$ & $\begin{array}{c}\text { FCR } \\
(\mathrm{kg} / \mathrm{d})\end{array}$ & Removals \\
\hline Control & 5.5 & $13.3^{\mathrm{a}}$ & 0.41 & $0.276^{\mathrm{a}}$ & $1.48^{\mathrm{b}}$ & 2 \\
PHM & 5.6 & $14.4^{\mathrm{c}}$ & 0.41 & $0.315^{\mathrm{c}}$ & $1.30^{\mathrm{a}}$ & 2 \\
PHM minus EO/OA & 5.5 & $14.2^{\mathrm{bc}}$ & 0.40 & $0.309^{\mathrm{bc}}$ & $1.29^{\mathrm{a}}$ & 4 \\
PHM minus KDF & 5.6 & $13.5^{\mathrm{ab}}$ & 0.38 & $0.284^{\mathrm{ab}}$ & $1.35^{\mathrm{a}}$ & 2 \\
SED & 0.49 & 0.34 & 0.013 & 0.012 & 0.033 & \\
$P$ value & 0.99 & 0.009 & 0.17 & 0.009 & $<0.001$ & $\chi^{2}(3) 1.22, P 0.748$ \\
\hline
\end{tabular}

a,b,c Means within a column with different superscripts differ significantly $(P<0.05)$; SED, standard error of difference of means

Conclusion The inclusion of $200 \mathrm{~g} / \mathrm{t}$ of PHM improved the growth performance of weaner pigs in the four weeks immediately post-weaning. With no difference observed in feed intake between treatments, but a significant improvement in feed conversion, this data is supportive of improvements in gut health indicated by other literature.

\section{References}

Liu Y, Espinosa CD, Abelilla JJ, Casas GA, Lagos LV, Lee SA, Kwon WB, Mathai JK, Navarro DMDL, Jaworski W and Stein HH 2018. Animal Nutrition. 4, 113-125.

Pluske JR, Turpin DL and Kim J-C 2018. Animal Nutrition. 4, 187-196. 


\section{Effects of Prevotella/Bacteroides ratio and dietary starch type on SCFAs concentrations,} composition of butyrate-producing bacteria and gene expression in finishing pigs

W Ren ${ }^{1,2}$, Y-H Luo ${ }^{1}$, B Yu ${ }^{1}$, D-W Chen ${ }^{1}, \mathrm{H}_{\text {Zhai }}{ }^{2}$ and M C Walsh ${ }^{3}$

${ }^{1}$ Sichuan Agriculture University, China, ${ }^{2}$ DSM (China) Animal Nutrition Research Center, China, ${ }^{3}$ DSM Nutritional

Products, Switzerland.

chendwz@sicau.edu.cn

Application This study provides us with information that will be used to elucidate the interaction between P/B ratio, dietary starch type and microbiota metabolites. It also provides fundamental knowledge that will facilitate studies such as how different enterotypes are interacted with diet and involved in host health.

Introduction The structure of host gut microbiota varies among individuals at genus level and can be clustered into socalled enterotypes (Arumugam, 2011). Each enterotype is defined by the most predominant bacteria and microbiota with high Prevotella-to-Bacteroides ratio (P/B) and low P/B ratio have been widely observed in the human gut. It has been proven in in vitro trials that microbes with different enterotypes produce different amounts and profiles of short chain fatty acids (SCFAs) from the same fibrous substrates (Chen et al., 2017; Wu et al., 2017). However, the direct gut health relevance of these enterotypes in vivo remains to be elucidated. Diet is known to strongly influence the composition of the gut microbiota as well as metabolites and starch with high amylose to amylopectin ratio is known to be largely fermented by microbiota in the colon, resulting in the production of SCFAs. We hypothesize that P/B ratio and dietary starch type influence the composition of butyrate-producing bacteria and SCFAs concentrations, which further modulate the expression of genes involved in SCFAs uptake and butyrate production in the colon of finishing pigs.

Material and methods After a pre-trial with 102 three-month old DYL (Duroc x Yorkshire x Landrace) pigs for the screening of enterotypes based on described method (Bergström et al., 2012), a total of 32 pigs were selected and fed in individual cages. Of these pigs, sixteen had high $\mathrm{P} / \mathrm{B}$ ratio (14) and another sixteen had low P/B ratio (2). The cornsoybean meal diets with high or low amylose/amylopectin ratio (17 or 0.20$)$ were prepared using different corn starch with high amylose or high amylopectin, respectively. The pigs with high and low $\mathrm{P} / \mathrm{B}$ ratio were randomly allocated into two groups ( 8 replicates per group) and fed with the two different diets respectively following a 2 x 2 factor design. All animals used in current study were healthy and did not receive any antibiotic treatment during the whole experimental period. On d 60 of the trial, all pigs were sacrificed and hindgut digesta and mucosa samples were collected for further analysis. The concentration of SCFAs (acetate, propionate and butyrate) in digesta were analysed using gas chromatography. The genomic DNA of digesta samples were extracted and used for the quantitative real-time PCR (qPCR) analysis of butyrate-producing bacteria (Clostridium cluster IV, Clostridium cluster XIVa and Faecalibacterium prausnitzii) and genes involved in butyrate production (butyryl-CoA:acetate CoA-transferase and butyrate kinase). mRNA was extracted from colonic mucosa samples and the expression of genes involved in SCFAs uptake (SMCT1, $M C T 1$ and SLC7A1) were also analysed using qPCR.

Results A significantly higher concentration of total SCFAs $(P=0.04)$ and propionate $(P<0.05)$ was observed in pigs with high $\mathrm{P} / \mathrm{B}$ ratio compared to those with low $\mathrm{P} / \mathrm{B}$. The concentration of propionate $(P=0.04)$ and butyrate $(P=0.02)$ was significantly higher in the colon of pigs fed with high amylose/amylopectin starch diets than those pigs fed with low amylose/amylopectin starch diets. A significant interaction between $\mathrm{P} / \mathrm{B}$ ratio and dietary starch type on the concentration of total SCFAs was also shown $(P=0.02)$, which tended to increase the concentration of acetate $(P=0.09)$ and propionate $(P=0.07)$ in the colon of the pigs. According to qPCR results, the number of Clostridium cluster XIVa in the colon of pigs with high $\mathrm{P} / \mathrm{B}$ ratio was significantly more than those pigs with low $\mathrm{P} / \mathrm{B}$ ratio $(P=0.03)$. Meanwhile, the number of Clostridium cluster XIVa was also found to be significantly higher in the colon of pigs fed with high amylose/amylopectin ratio diet compared to those fed with low amylose/amylopectin ratio diet $(P<0.01)$, accompanied with significantly higher expression of butyryl-CoA:acetate CoA-transferase $(P<0.01)$ and butyrate kinase $(P=0.04)$ genes, as well as the expression of SMCT1 gene in the colonic mucosa $(P=0.03)$.

Conclusion In accordance with our hypothesis, this study showed that the concentration of SCFAs in the colon of pigs can be affected by both $\mathrm{P} / \mathrm{B}$ ratio and dietary starch type. High $\mathrm{P} / \mathrm{B}$ ratio in the colon of pigs may tended to increase the number of butyrate-producing bacteria, while both the composition and activity of butyrate-producing bacteria could be modulated by the amylose/amylopectin ratio in the diet. Besides, ingestion of diet with high amylose/amylopectin ratio could enhance the mucosal uptake of SCFAs in the colon of pigs. Combined, our data provide an enhanced understanding of the interaction between $\mathrm{P} / \mathrm{B}$ ratio, dietary starch type and microbiota metabolites.

\section{References}

Arumugam M, Raes J, Pelletier E, Le Paslier D, Yamada T, Mende DR ... Bork P 2011. Nature. 473, $174-180$.

Chen T, Long W, Zhang C, Liu S, Zhao L and Hamaker BR 2017. Scientific Reports. 7, 2594.

Wu Q, Pi X, Liu W, Chen H, Yin Y, Yu HD, Wang X and Zhu L 2017. Anaerobe. 48, 206-214.

Bergström A, Licht TR, Wilcks A, Andersen JB, Schmidt LR, Grønlund HA, Vigsnæs LK, Michaelsen KF and Bahl MI 2012. FEMS Microbiology Letters. 337, 38-47. 


\section{Use of novel photoperiod schedules for stimulating weaner performance}

J R Craig $^{1}$, R S Morrison ${ }^{1}$, D J Henman ${ }^{1}$, C J Brewster ${ }^{1}$, E M de Ruyter ${ }^{1,2}$ and K S O'Halloran ${ }^{1}$

${ }^{1}$ Rivalea Australia Pty. Ltd., Australia; ${ }^{2}$ Present affiliation: Charles Sturt University, Australia jcraig@rivalea.com.au

Application Producers can continue to utilise standard lighting schedules (typically $8 \mathrm{~h}$ on, $16 \mathrm{~h}$ off) in their weaner housing without impacting the post-weaning performance of pigs from weaning to 9 weeks of age.

Introduction Despite a plethora of information available to pork producers on management and feeding strategies for rearing weaned pigs, weaner pigs still commonly have a post-weaning growth setback. This setback can have a longstanding effect in that low growth rate during the weaner phase usually persists to slaughter (Dunshea et al., 2003). One possible risk factor that may be contributing to feed intake after weaning is the lighting schedule (Bruininx et al., 2002). The hypothesis of this experiment was that lighting schedule and source will impact post-weaning feed intake and growth performance of pigs.

Material and methods A total of 4017 commercial weaner pigs (Large White $\mathrm{x}$ Landrace $\mathrm{x}$ Duroc Synthetic, Primegro $^{\text {TM }}$ Genetics, Corowa, NSW) split evenly across sexes were followed from weaning at 4 weeks of age (14-15 pigs per pen) for the subsequent 5 weeks, between June and October, 2017. Treatments, split between sheds of similar design and environment, as to not allow interference between lighting schedules, were: (a) fluorescent lighting (40.6 $\pm 21.5 \mathrm{~lx}$ ) $8 \mathrm{~h}$ on (from 0630 to $1430 \mathrm{~h}$ ) and $16 \mathrm{~h}$ off each day from weaning (d 0); (b) fluorescent lighting continuously on for $48 \mathrm{~h}$ after weaning, then on for $18 \mathrm{~h}(0630$ to $0030 \mathrm{~h}$ ), and off for $6 \mathrm{~h}$ each day; and (c) BioLumen lighting (38.0 \pm 17.6 lx; 1500 NP White; AgriRay, BioLumen Lighting, UK) on for $48 \mathrm{~h}$ after weaning, then on for $18 \mathrm{~h}(0630$ to 0030 h), and off for $6 \mathrm{~h}$ each day. There were approximately 90 pen replicates of each treatment and each pen was weighed at d 0, 7, 21 and 35 with pen feed intakes measured during these periods. Growth performance data were analysed using the UNIVARIATE procedure (IBM SPSS Statistics for Windows, v24.0. Armonk, NY) with lighting type and side of the shed as fixed factors, replicate as a blocking factor, and pen as the experimental unit. Neither sex, side of the shed, nor any of their interactions with lighting type were significant $(P \geq 0.05)$ for any traits and were therefore left out of the model. Piglet mortality was analysed as a categorial variable using Pearson's $\chi^{2}$.

Results Lighting treatment did not significantly affect average pig body weight (BW), average daily feed intake (ADFI), average daily gain (ADG) or feed conversion ratio (FCR) over the entire weaner period $(P \geq 0.05$; Table 1$)$, or during any of the intermediate points (d 0-7, d 7-21, or d 21-35). Mortality rate was not different $(P \geq 0.05)$ between treatment groups.

Table 1. Growth performance (average daily gain, ADG; average daily feed intake, ADFI; feed conversion ratio, FCR; average body weight, BW) of weaner pigs under 3 different lighting schedules from weaning (d 0) until 5 weeks after weaning (d 35).

\begin{tabular}{|c|c|c|c|c|}
\hline & \multicolumn{3}{|c|}{ Lighting treatment } & \multirow[b]{2}{*}{$P$ value } \\
\hline & Fluorescent $8 \mathrm{~h}$ & Fluorescent $18 \mathrm{~h}$ & BioLumen $18 \mathrm{~h}$ & \\
\hline BW d 0 (kg) & $7.6 \pm 0.2$ & $7.6 \pm 0.2$ & $7.7 \pm 0.2$ & 0.92 \\
\hline BW d $35(\mathrm{~kg})$ & $22.2 \pm 0.3$ & $22.3 \pm 0.3$ & $21.9 \pm 0.3$ & 0.85 \\
\hline ADG d 0-35 (g/d) & $426 \pm 5$ & $425 \pm 5$ & $416 \pm 5$ & 0.37 \\
\hline ADFI d 0-35 (g/d) & $568 \pm 8$ & $566 \pm 8$ & $547 \pm 8$ & 0.38 \\
\hline FCR d 0-35 (g:g) & $1.33 \pm 0.01$ & $1.33 \pm 0.01$ & $1.33 \pm 0.01$ & 0.93 \\
\hline Mortality d 0-35 (\%) & 2.4 & 3.1 & 2.3 & 0.38 \\
\hline
\end{tabular}

Conclusion There was no significant improvement in any performance parameters of pigs housed in different lighting environments. It is therefore recommended that pork producers install and implement standard ( $8 \mathrm{~h}$ on, $16 \mathrm{~h} \mathrm{off})$ lighting schedules in their weaner facilities, in preference to more complex (and often more expensive) schedules.

Acknowledgements Supported in part by Australian Pork Limited (Project number 2016/070) and the Australian Government Department of Agriculture and Water Resources.

\section{References}

Bruininx EMAM, Heetkamp MJW, van den Bogaart D, van der Peet-Schwering CMC, Beynen AC, Everts H, den Hartog LA and Schrama JW 2002. Journal of Animal Science. 80, 1736-1745.

Dunshea FR, Kerton DK, Cranwell PD, Campbell RG, Mullan BP, King RH, Power GN and Pluske JR 2003. Australian Journal of Agricultural Research. 54, 363-370. 


\section{Sorting newly weaned pigs by body weight in a commercial setting takes more time and does not improve performance}

D L Turpin $^{1,2}$ and J R Pluske ${ }^{1}$

${ }^{1}$ Murdoch University, Australia, ${ }^{2}$ Pork Innovation Western Australia, Australia

Diana.Turpin@murdoch.edu.au

Application Not sorting pigs by body weight at weaning is an acceptable practice for medium and heavy pigs.

Introduction Sorting piglets into body weight groups (light, medium, and heavy) is a common management practice at weaning. However, in studies using small group sizes, it is recognised that fights are more frequent and severe between pigs of a similar size (Tindsley and Lean, 1984; Andersen et al., 2000). Therefore, mixing pigs according to body weight at the time of weaning might simply add additional labour costs without improving production, welfare and health outcomes. This study tested the hypothesis that sorting pigs according to body weight at the time of weaning will take longer and will not improve post-weaning performance, and that the incidence of fighting will be higher in homogeneous groups. This study was conducted in a commercial setting with larger group sizes than have previously been explored.

Material and methods A total of 1280 newly-weaned pigs were allocated to one of four treatments; homogenous, where pigs were divided into either i) light, ii) medium or iii) heavy weight classes, and iv) heterogeneous, where pigs from all weight classes were mixed together $(n=8)$. Heterogeneous groups were achieved by opening the gate and letting the first 40 pigs through. Weaner housing for each treatment group consisted of pens of 40 pigs at a stocking density of $0.3 \mathrm{~m}^{2}$. Weaning day ( $\mathrm{d} 0$ ) average pen weights were: light $4.5 \pm 0.1$, medium $6.0 \pm 0.1$, heavy $7.8 \pm 0.1$ and heterogeneous 6.5 $\pm 0.1 \mathrm{~kg}$ (mean $\pm \mathrm{SE})(P<0.001)$. A commercial weaner diet was offered ad libitum. Average daily gain (ADG) and average daily feed intake (ADFI) were compared on different days throughout the nursery stage between treatments using the GLM procedures of SPSS (IBM SPSS Statistics for Windows, v24.0. Armonk, NY) with pen as the experimental unit. A mixed linear model compared scratch scores (0 to 3 scale) on the ears, head and flank between four randomly selected individuals per pen (categorised as light, medium or heavy by eye in the heterogeneous pens). Removals due to death or treatment were recorded and compared using a Chi-squared analysis.

Results It took 382 full-time equivalent (FTE) minutes to sort pigs by weight on d 0 versus 36 FTE minutes to not sort. There were no treatment differences in performance between homogenous (heavy, medium and light pens combined) and heterogeneous groups (Figure 1A, 1B, $P>0.05$ ). Day 42 body weights were $25.3 \pm 0.5$ versus $26.0 \pm 0.9 \mathrm{~kg}$ for pooled homogenous and heterogeneous pens respectively $(P>0.05)$. Scratch scores were compared across the three different weight categories within the homogeneous and heterogeneous groups (Figure 1C). Scratch levels were highest $24 \mathrm{~h}$ after weaning, followed by d 6 and then $\mathrm{d} 0(P<0.001)$. Overall, the heavy homogenous pigs had more scratches than the heavy mixed pigs $(P<0.05)$, which is consistent with previous results (Rushen, 1987). However, the light homogenous pigs had less scratches than their light counterparts in heterogeneous pens $(P<0.001)$. Removal rate tended to be higher in the homogenous groups $(8.6 \%$ vs $5.2 \%$ for homogeneous and heterogeneous groups respectively, $P<0.1)$.
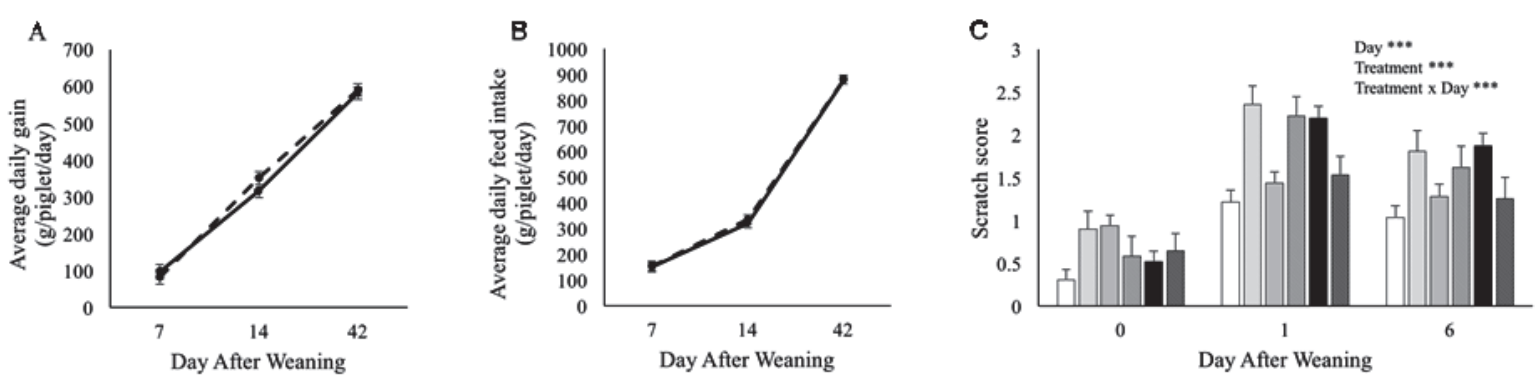

Figure 1. Post weaning ADG (A) and ADFI (B) for homogeneous (solid line, $n=24$ ) versus heterogeneous (dashed line, $n=8$ ) pens, and scratch scores (C) for individuals within different weight categories for homogenous ( $\square$ light, $\square$ medium, -heavy) and heterogeneous ( $\square$ light, medium, wheavy) groups $(n=24)$.

Conclusion Sorting pigs by body weight (BW) at weaning took longer with no improvement in post-weaning performance. Scratch score results suggest that light pigs may still benefit from being housed with similar sized pigs to reduce potential bullying or lack of access to resources, while BW asymmetry in pens with pigs from the heavier cohort may reduce injury scores possibly due to a quicker establishment of hierarchy.

Acknowledgements Supported in part by the Agricultural Produce Commission, Pork Producers' Committee.

\section{References}

Andersen IL, Andenæs H, Bøe KE, Jensen P and Bakken M 2000. Applied Animal Behaviour Science. 68, 107-120. Tindsley WEC and Lean IJ 1984. Applied Animal Behaviour Science. 12, 79-92.

Rushen J 1987. Canadian Journal of Animal Science. 67, 951-960. 
Farm differences, but not novel dietary ingredients, affects carcass and meat quality of pork $\underline{\text { R J Smits }}{ }^{1}$, A K Lealiifano ${ }^{1}$, R Aitken ${ }^{1}$, H A Channon ${ }^{2}$ and P Smith ${ }^{2}$

${ }^{I}$ Rivalea Australia Pty Ltd, Australia, ${ }^{2}$ Australian Pork Ltd, Australia

rsmits@rivalea.com.au

Application The use of novel feed ingredients, such as macadamia meal, is unlikely to improve pork carcass quality, whereas pig housing and production rearing systems and breed can contribute to carcass quality improvements.

Introduction Taste and tenderness are eating quality attributes that are influenced by genetics and diet, amongst other pre- and post- slaughter factors (Channon et al., 2018a). Outdoor free-range housing combined with the use of traditional breeds such as Duroc and Berkshire are often sought by consumers due to marketable characteristics of taste, juiciness and flavour, whilst "novel" ingredients such as macadamia are used by some producers to enhance value. However, other published research concluded that typical corn-soy diet formulations compared to wheat or sorghum-wheat diets had only minimal impact on carcass and pork quality (Channon et al., 2018b). We tested the hypotheses that different production rearing systems with representative breeds and supplementing a finisher diet with macadamia meal results in differences in objective and sensory pork quality parameters and changes the fatty acid profile of pork and pork fat.

Material and methods The study was conducted on 36 female pigs in total sourced from two farms (Primegro ${ }^{\mathrm{TM}}$ Genetics, Corowa, NSW). On Farm A, pigs of Berkshire x Duroc breed (BxD) were reared from 9 weeks of age on straw bedding in two ecoshelter pens at Gre Gre, VIC $(\mathrm{n}=18)$. On Farm B, pigs of Large White $\mathrm{x}$ Landrace $\mathrm{x}$ Duroc synthetic, (COMM) were housed in two partially slatted pens in a conventional grower-finisher shed at Corowa, NSW ( $\mathrm{n}=18)$. At both farms, pigs at 15 weeks of age were fed either an un-supplemented finisher diet or the same diet with a supplement (1:1) of macadamia meal (13.8\% crude fat; Proteco Oils, Kingaroy, QLD). All pigs were transported to the Corowa abattoir at 21 weeks of age and slaughtered at the same time the following day. Carcasses were measured for weight (HSCW, Trim 1), backfat depth at the P2 site, muscle $\mathrm{pH}$ at $45 \mathrm{mins}, 3 \mathrm{~h}, 6 \mathrm{~h}$, and $24 \mathrm{~h}$ after slaughter. Carcasses were boned 24h later and the loin (M. longissimus thoracis et lumborum) samples were collected for objective and sensory quality as per Channon et al. (2018c). All data was analysed by GLM ANOVA using Farm and Diet as main effects or interactions (IBM SPSS Statistics for Windows, v25.0. Armonk, NY).

Results At a similar age of 21 weeks, carcass weight and P2 fatness were higher $(P<0.01)$ in Farm A carcasses compared to Farm B $(83.5 \pm 1.9 \mathrm{~kg}, 18.8 \pm 1.2 \mathrm{~mm} v s 75.3 \pm 1.5 \mathrm{~kg}, 10.2 \pm 0.5$; mean $\pm \mathrm{SE})$, respectively. Intramuscular fat (IMF) concentration was higher in loin samples from Farm A (Table 1). Dietary macadamia meal had no effect on loin n-3 or n-6 fatty acids levels, however there was a tendency for a higher C16:1 n-7 (palmitoleic acid) concentration in pork from pigs fed macadamia meal compared to the un-supplemented diet (3.66 vs 3.34\%, $P=0.051$ ). There was no effect of Diet or Farm $\mathrm{x}$ Diet interactions on carcass weight, fatness, carcass $\mathrm{pH}$ or any pork quality parameters. Farm A loins were darker, redder, and more tender than Farm B. There were Farm differences in fatty acid profile (\% dry matter) for the n3 fatty acids C18:3 (0.39 vs 0.46\%), C22:5 (0.35 vs 0.59\%) and C22:6 (0.21 vs 0.31\%) in Farm A compared to Farm B pork loins $(P<0.010)$. The concentration of n-6 fatty acids $(P<0.010)$ was also lower in loins from Farm A: C18:2 $(7.65$ vs $11.2 \%), \mathrm{C} 18: 2$ (0.08 vs $0.12 \%)$ and $\mathrm{C} 20: 4$ (2.01 vs 3.19\%) respectively.

Table 1. Mean meat quality values of pork loins taken from Farm A (Berkshire x Duroc, straw based ecoshelter) and Farm B (Large White x Landrace x Duroc, conventional housing) lines as main Farm effects. Diet and Farm x Diet interactions for all parameters were not significant $(P>0.10)$.

\begin{tabular}{|c|c|c|c|c|}
\hline & Farm A & Farm B & SEM & $P$ value \\
\hline Loin $\mathrm{pH}_{24}$ & 5.41 & 5.25 & 0.02 & 0.001 \\
\hline Drip Loss (\%) & 6.6 & 7.7 & 0.5 & 0.289 \\
\hline Loin IMF (\% wet) & 2.4 & 1.4 & 0.1 & $<0.001$ \\
\hline Shear force $(\mathrm{kg})$ & 4.26 & 5.24 & 0.15 & 0.028 \\
\hline Colour L* & 47.2 & 49.6 & 0.5 & 0.021 \\
\hline $\mathrm{a}^{*}$ & 7.4 & 5.7 & 0.2 & $<0.001$ \\
\hline$b^{*}$ & 2.8 & 2.6 & 0.15 & 0.398 \\
\hline
\end{tabular}

Conclusion Pork quality parameters including colour, tenderness and IMF will differ when carcasses are supplied from farms of different production systems and breed. The use of macadamia meal as a diet supplement had no effect on carcass or meat quality measures, however it specifically increased the n-7 fatty acid, palmitoleic acid. This may impact on sensory assessment of flavour.

Acknowledgements Supported in part by Australian Pork Limited and Rivalea Australia Pty Ltd.

\section{References}

Channon HA, D’Souza DN, Jarrett RG, Lee GSH, Watling RJ, Jolley JYC and Dunshea FR 2018a. Meat Science. 144, 186-192.

Channon HA, D'Souza DN, and Dunshea FR 2018b. Meat Science. 135, 94-101.

Channon HA, D'Souza DN, and Dunshea FR 2018c. Meat Science. 136, 104-115. 


\section{Recognition of high-speed aggressive interactions of pigs on group level by depth sensor}

C Chen ${ }^{1,2}$, W Zhu ${ }^{1}$, D Liu ${ }^{2}$, J Steibel $^{3}$, J Siegford ${ }^{3}$, T Norton $^{2}$

${ }^{1}$ Jiangsu University, China, ${ }^{2} \mathrm{KU}$ Leuven, Belgium, ${ }^{3}$ Michigan State University, USA.

tomas.norton@kuleuven.be

Application This research optimises the approach of detecting aggressive behaviour based on characteristics of pig activity, which can be used to automatically quantify the level of aggression in the herd. This work assumes the number of moving pixels of aggressive behaviours is usually more than the moving pixels of other behaviours.

Introduction Compared to traditional methods of manually observing aggression, computer vision technology has the capacity to provide non-intrusive, uninterrupted, rapid and objective monitoring (Bracke et al., 2002). In order to combine advantages of group analysis and 3D cameras, this work attempts to recognise aggressive pig behaviours on group level by using a depth sensor. In the previous studies of aggressive behaviours of pigs based on group analysis (Viazzi et al., 2014; Oczak et al., 2014), all pigs' moving pixels were considered. However, the moving pixels caused by non-aggressive pigs will influence the accuracy of the aggression recognition. Hence, the objective of this study is to remove the influence of these non-aggressive pixels by setting the threshold of moving pixels in order to improve the accuracy of aggression recognition.

Material and methods In this work two experiments were performed. In each experiment, 8 pigs from 3 different pens were mixed for $2 \mathrm{~d}$ with $8 \mathrm{~h}$ of video recorded each day. Firstly, frame difference method was used to obtain moving pixels. Secondly, moving pixels with non-aggressive behaviours were removed by setting threshold of connected area. Number of filtered moving pixels in each frame were summed and defined as improved aggressive pixels (IAP). After dividing all videos into 3 second videos, mean, variance and standard deviation of IAP in each unit were extracted as features. The idea of Support Vector Machine (SVM) is to map features to a hyperplane, which is used to maximise the distance between these features in order to classify them. Finally, SVM was used to classify these features in order to recognise aggressive behaviours. These aggressive behaviours include head to head knocking, head to body knocking, parallel pressing, inverse parallel pressing, neck biting, body biting and ear biting.

Results The proposed algorithm can be used to recognise aggressive behaviours from a series of non-aggressive behaviours with good accuracy, sensitivity and specificity, 96.9\%, 97.8\% and 95.9\%, respectively.

Table 1. Classification results of SVM on test set and evaluation parameters of the proposed algorithm.

\begin{tabular}{ccccccc}
\hline TP units & FN units & FP units & TN units & Accuracy & Sensitivity & Specification \\
\hline 577 & 13 & 24 & 566 & $96.9 \%$ & $97.8 \%$ & $95.9 \%$ \\
\hline
\end{tabular}
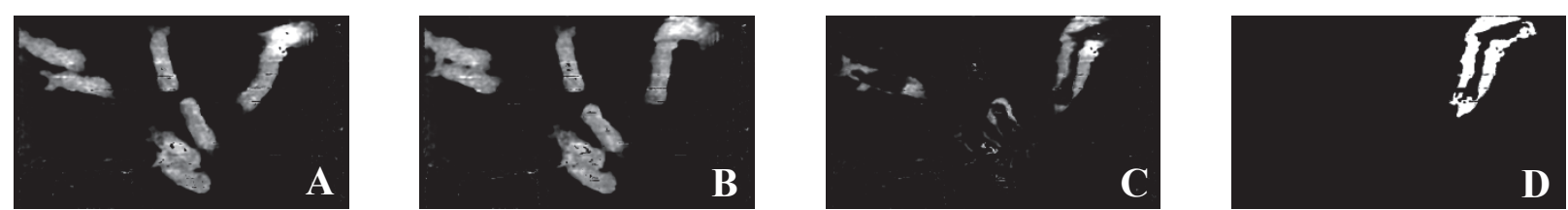

Figure 1. The former (A) and latter (B) depth images. Frame difference method was then conducted to extract moving pixels between the former and latter depth images $(\mathrm{C})$. Finally, setting the threshold of moving pixels and binarisation were used to extract filtered moving pixels (D).

Conclusion Setting the threshold of moving pixels can be used to remove the influence of non-aggressive pixels on the accuracy of aggression recognition. Mean, variance and standard deviation of IAP are powerful features. Using SVM to classify features can obtain higher accuracy. Future work could be focused on the recognition of aggressive behaviours during night.

Acknowledgements Supported in part by the National Natural Science Foundation of China (31872399), and the USDA National Institute of Food and Agriculture (2017-67007-26176).

\section{References}

Bracke MB, Metz JH, Spruijt BM and Schouten WG 2002. Journal of Animal Science. 80, 1835-1845.

Viazzi S, Ismayilova G, Oczak M, Sonoda LT, Fels M, Guarino M, Vranken E, Hartung J, Bahr C and Berckmans D 2014. Computers and Electronics in Agriculture. 104, 57-62.

Oczak M, Viazzi S, Ismayilova G, Sonoda LT, Roulston N, Fels M, Bahr C, Hartung J, Guarino M, Berckmans D and Vranken E 2014. Biosystems Engineering. 119, 89-97. 


\section{Mixing sows into group lactation housing shows marked differences between multisuckle and} sow separation systems

E C Greenwood $^{1}$, J van Dissel ${ }^{1}$, J Rayner ${ }^{2}$, P E Hughes ${ }^{3}$ and W H E J van Wettere ${ }^{1}$

${ }^{1}$ The University of Adelaide, Australia, ${ }^{2}$ South Australian Research and Development Institute, Australia, ${ }^{3}$ Paul Hughes Consulting, Australia

emma.greenwood@adelaide.edu.au

Application Group housing during late lactation may improve sow and piglet welfare by enabling natural behaviours. However, for these systems to be viable, sow and litter productivity must at least be maintained, compared to farrowing crate systems. This pilot provides evidence that mixing in lactation, in the presence of piglets, may increase sow welfare, while maintaining production targets, however, different methods of mixing in lactation create very different results.

Introduction In Australia, the majority of breeding sows are housed in farrowing crates prior to and during parturition and this is where they remain until weaning. Once weaning is complete, there are two main management strategies employed; either the sows are mixed into groups or housed individually and mixed in the first week after insemination. Group housing during late lactation may improve sow and piglet welfare by enabling natural behaviours (such as sowregulated lactation, social interaction and a more gradual weaning). Sow and litter productivity must be improved, or at least maintained, compared to conventional systems for these systems to be considered. We aimed to: 1) compare the effects of mixing sows in lactation, at weaning or after insemination, on aggression and stress; and, 2) determine the effects of lactation housing on the piglet growth and welfare. We hypothesised that mixing in lactation would decrease aggression in sows upon mixing and have no negative effects on piglet welfare.

Material and methods The pilot study used 120 multiparous Large White x Landrace sows and 54 focal litters. The sows were mixed into groups of six and allocated to four treatments. MS: (multisuckle) group-housed sows and litters, mixed from crates on d 21 of lactation, SEP; sows separated from litter and housed in groups, with piglets left in the crate, for seven hours daily and then returned to home crate (from d 21 lactation), WEAN: mixed at weaning (d 28 lactation) and MAI: mixed after post-weaning artificial insemination (AI) from stalls ( $4 \pm 1 \mathrm{~d}$ after last AI). The WEAN and MAI are controls, these treatments have the same weaning experience for piglets. Sow behaviour (observed continuously for $6 \mathrm{~h}$, between 0700 - $1300 \mathrm{~h}$, once all sows were in groups), saliva for free salivary cortisol concentration (sows chewed cotton swabs) and injury counts (modified assessment described by Karlen et al. (2007), injuries counted and summed) were measured on the $\mathrm{d}-1$, the day of mixing and $\mathrm{d} 1$ and 6 after mixing. Piglets were weighed, injury scored (1-3 score adapted from Widowski et al., 2003), and bloods taken for cortisol, on lactation d 20, 21, 24 and 28 (weaning day) and the day after weaning. Data were analysed using a general linear mixed model (IBM SPSS Statistics for Windows, v20.0. Armonk, NY). When transformation was required, the back-transformed data and non-transformed SEM is provided.

Results Aggressive behaviours (fights, bites and knocks) were reduced in MS (Table 1). MS sows had no fights on d 1 and 6 after mixing and had more piglets in the subsequent farrowing [MS; $3.7 \pm 0.1$ Sqrt total born (13.7) SEP; $3.5 \pm 0.1$ (12.2) WEAN; $3.5 \pm 0.1$ (12.2) MAI; $3.3 \pm 0.1$ (11.0), P < 0.05)]. Piglet weight, cortisol and mortality were unaffected by treatment $(P>0.05)$. MS piglets had greater injury scores on d 24 of lactation (lactation mixing began d 21) and lower injuries on the day of weaning (lactation $\mathrm{d} 28)$ and the day after weaning $(P>0.001)$ than the other treatments.

Table 1. Effect of mixing treatment (means \pm SEM) of gestating sows (MS, multisuckle; SEP, sows seperated; WEAN, mixed at weaning; MAI, mixed after AI) on measures of aggression delivered per hour in the days after mixing.

\begin{tabular}{lcccccc}
\hline Behaviour & MS & SEP & WEAN & MAI & Trans. & $P$ value \\
\hline Fights/hour, per sow & $0.08^{\mathrm{a}} \pm 0.04$ & $0.20^{\mathrm{b}} \pm 0.03$ & $0.15^{\mathrm{ab}} \pm 0.04$ & $0.11^{\mathrm{ab}} \pm 0.03$ & $\operatorname{Lg}^{10}(1+\mathrm{x})$ & $<0.05$ \\
Fight duration, seconds & $3.0^{\mathrm{a}} \pm 7.5$ & $15.6^{\mathrm{b}} \pm 7.0$ & $16.9^{\mathrm{b}} \pm 7.5$ & $16.5^{\mathrm{b}} \pm 7.0$ & $\operatorname{Lg}^{10}(1+\mathrm{x})$ & $<0.05$ \\
Bites/hour, per sow & $0.4^{\mathrm{a}} \pm 0.2$ & $2.1^{\mathrm{b}} \pm 0.2$ & $1.0^{\mathrm{c}} \pm 0.2$ & $0.8^{\mathrm{c}} \pm 0.2$ & $\operatorname{Lg}^{10}(1+\mathrm{x})$ & $<0.0001$ \\
Knocks/hour, per sow & $0.4^{\mathrm{a}} \pm 0.3$ & $1.5^{\mathrm{b}} \pm 0.3$ & $1.1^{\mathrm{ab}} \pm 0.3$ & $0.7^{\mathrm{ab}} \pm 0.3$ & $\operatorname{Lg} 10$ & $<0.05$ \\
\hline
\end{tabular}

${ }^{\mathrm{a}, \mathrm{b}, \mathrm{c}}$ Means within a row with different superscripts differ significantly $(P<0.05)$; Trans, transformation used for statistical analysis.

Conclusion Mixing sows during lactation, in the presence of their piglets, reduced aggression and injuries, improved subsequent reproduction and had no apparent ill effects on piglets. Injuries were greater after mixing in these piglets but lower around weaning. The mixing of sows during lactation, separated from piglets, increased aggression levels. Due to the substantial differences between the results from the lactation treatments, we cannot support or dispute the hypothesis that mixing in lactation decreases sow aggression, and so further work is required in this area.

Acknowledgements Supported in part by Pork CRC Ltd and The University of Adelaide. The UWA Institute of Agriculture are thanked for their assistance in analysing free cortisol concentrations in saliva.

\section{References}

Karlen GAM, Hemsworth PH, Gonyou HW, Fabrega E, Strom AD and Smits RJ 2007 Applied Animal Behaviour Science 105, 87-101.

Widowski TM, Cottrell T, Dewey CE and Friendship RM 2003 Journal of Swine Health and Production 11, $181-185$. 


\section{Pronounced inter-individual variation in plasma cortisol response to fluoxetine hydrochloride in the pig}

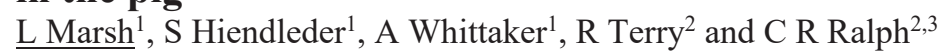

${ }^{1}$ The University of Adelaide, Australia, ${ }^{2}$ South Australian Research and Development Institute, Australia, ${ }^{3}$ Present address: Agersens, Australia

laura.latimer-marsh@adelaide.edu.au

Application Circulating cortisol is not a reliable marker to validate SSRI effects on the HPA axis in the pig.

Introduction It is widely recognised that assessment of animal welfare should include measures of both negative and positive emotional states (Boissy et al., 2007). Selective serotonin reuptake inhibitors (SSRI's) have been used to treat several psychological conditions, including depression and anxiety, by increasing serotonergic tone in regions of the brain associated with reward processing. Administration of SSRI's have been shown to activate the HPA axis in humans, sheep and rodents (Bhagwagar et al., 2002; Broadbear et al., 2004). As such, an increase in plasma cortisol can be used as a biomarker for effective delivery of the SSRI as well as effective activation of the brain by the SSRI in these species. In the present experiment we tested whether measurement of circulating cortisol following SSRI treatment could be used to determine effective delivery of administered SSRI to the brain of the pig. We hypothesised that through pharmacological manipulation of the serotonergic system in pigs, by SSRI, we would activate the HPA axis resulting in cortisol production. A peripheral cortisol response provides evidence of successful entry of SSRI to the brain target regions, including those associated with positive emotional state.

Material and methods Eight Large White x Landrace 18 week old gilts were randomly allocated to either the saline or SSRI treatment $(n=4$ gilts per treatment). SSRI treated gilts were administered a single intravenous dose of $100 \mathrm{mg}$ fluoxetine hydrochloride suspended in $10 \mathrm{ml}$ of saline whilst the saline treated gilts were administered intravenous $10 \mathrm{ml}$ saline. One week prior to the commencement of the experiment, gilts were transferred to individual pens and given sweet treats to aid in habituation to the environment. Indwelling ear vein catheters were inserted the day prior to treatment administration. Blood samples were collected every 15 minutes for one hour prior to and six hours post treatment and plasma cortisol concentration determined by radioimmunoassay. Data were analysed using a general linear model with time as the repeated-measure (IBM SPSS Statistics for Windows, v24.0. Armonk, NY).

Results Mean cortisol results indicated a significant treatment by time interaction where pigs exposed to SSRI displayed greater plasma cortisol (50-145 ng/mL), compared with control $(25-50 \mathrm{ng} / \mathrm{mL})$ between $15-165 \mathrm{mins}$ following treatment $(P<0.05)$. However, the individual cortisol profiles after SSRI treatment showed high inter-individual variation and apparent dichotomy in response to SSRI (Table 1).

Table 1. Mean, minimum and maximum cortisol concentration in response to intravenous fluoxetine hydrochloride or saline.

\begin{tabular}{ccccc}
\hline Animal & Treatment & Mean cortisol $(\mathrm{ng} / \mathrm{mL})$ & Min cortisol $(\mathrm{ng} / \mathrm{mL})$ & Max cortisol $(\mathrm{ng} / \mathrm{mL})$ \\
\hline 1 & Saline & 17.1 & 0.4 & 96.3 \\
2 & Saline & 21.0 & 0.2 & 59.5 \\
3 & Saline & 24.4 & 0.7 & 57.2 \\
4 & Saline & 25.1 & 1 & 79.2 \\
5 & SSRI & 70.1 & 5.3 & 214.2 \\
6 & SSRI & 33.5 & 0.7 & 102.1 \\
7 & SSRI & 75.8 & 3 & 228.3 \\
8 & SSRI & 31.0 & 0.9 & 178.4 \\
\hline
\end{tabular}

Conclusion These results provide information on the effectiveness of using circulating cortisol as a biomarker for SSRI efficacy in the pig. The SSRI altered HPA axis activity and mean cortisol concentrations were significantly higher than controls and therefore the hypothesis is supported. However, the relationship between the neurobiology of emotion and HPA activity is complex and individual differences in cortisol in response to SSRI may preclude the use of this approach in assessing the effectiveness of using SSRI to activate regions of the brain associated with reward processes and altered HPA activity.

Acknowledgements Supported in part by Australian Pork Limited. We thank Jessica Zemitis, Lisa McKenny, Serena Barnes and Tanya Nowland for their technical assistance in data collection.

\section{References}

Bhagwagar Z, Hafizi S and Cowen P 2002. Psychopharmacology. 163, 118-120.

Boissy A, Manteuffel G, Jensen MB, Moe RO, Spruijt B, Keeling LJ, Winckler C, Forkman B, Dimitrov I, Langbein J, Bakken M, Veissier I and Aubert A 2007. Physiology \& Behavior. 92, 375-397.

Broadbear J, Hutton L, Clarke I and Canny B 2004. Psychopharmacology. 171, 450-457. 


\title{
Hessian stimulates nest building behaviour in sows and the provision of straw improves traits important for piglet survival
}

\author{
L A McKenny ${ }^{1}, \mathrm{~K}$ J Plush ${ }^{3}$, T L Nowland ${ }^{1,2}$ and $\mathrm{W}$ H E J van Wettere ${ }^{2}$ \\ ${ }^{1}$ South Australian Research and Development Institute, Australia, ${ }^{2}$ The University of Adelaide, Australia, ${ }^{3}$ SunPork \\ Group, Australia \\ william.vanwettere@adelaide.edu.au
}

Application The use of straw as a nest building substrate is beneficial for crated sows and piglets, whilst hessian promotes nest building pre-partum.

Introduction Pre-partum nesting in sows is an intrinsic behaviour and is stimulated both internally (via hormones) and externally (via feedback from the environment). Inhibition of this behaviour can cause stress and frustration in the sow and may result in adverse production outcomes. The aim of this study was to investigate the impact of hessian sacks and straw on peri-partum sow behaviour. We hypothesised that; (1) The provision of nesting materials would reduce stress associated with confinement during the nest-building phase; (2) The provision of a man-made material (hessian sack) would be an effective alternate material to straw for farrowing sows.

Material and methods First and second parity sows $(1.7 \pm 0.1)$ were randomly allocated to one of four treatments at $d$ $110 \pm 2$ of gestation: sows housed in an open 360 farrowing pen with $2 \mathrm{~kg}$ chopped straw provided daily in the lead up to farrowing, with the pen closed at placental expulsion (STRAW OPEN; n=15), sows housed in a closed 360 farrowing pen with $2 \mathrm{~kg}$ chopped straw provided daily in the lead up to farrowing (STRAW CLOSED; $\mathrm{n}=14$ ), sows housed in a closed 360 farrowing pen with hessian sacks attached to the front bars via chains in the lead up to farrowing (HESSIAN; $\mathrm{n}=15$ ), sows housed in a closed 360 farrowing pen with no nesting substrate provided (CONTROL; $\mathrm{n}=13$ ). A subset of individual sow videos (CONTROL; n=7, HESSIAN; n=6, STRAW CLOSED; $n=6$, and STRAW OPEN; $n=6$ ) were analysed for pre-farrow behaviour ( $-18 \mathrm{~h}$ pre-farrow). Blood samples were collected 4 hourly from $-24 \mathrm{~h}$ until farrowing completion and pooled for cortisol analysis. Using SPSS (IBM SPSS Statistics for Windows, v21.0. Armonk, NY), behaviour analyses were conducted using a Generalised linear mixed model with poisson distribution and Cortisol analysed using a Genlin model.

Results Pooled plasma cortisol concentration was lower overall in the STRAW CLOSED treatment than any other treatment (Table 1). The HESSIAN, STRAW CLOSED and STRAW OPEN sows all performed more nosing behaviour towards the straw prior to farrowing. The number of posture changes during farrowing was lowest in the HESSIAN and STRAW CLOSED treatments. The highest incidence of piglets needing to be saved from potential crushing were observed in the STRAW OPEN treatment. Piglets born to confined sows provided with straw gained more weight to $24 \mathrm{~h}$ of age than their counterparts.

Table 1. Behavioural and physiological outcomes of sows and piglets following pre-partum treatments. Data are expressed as means \pm SEM

\begin{tabular}{|c|c|c|c|c|c|}
\hline & CONTROL & HESSIAN & $\begin{array}{l}\text { STRAW } \\
\text { CLOSED }\end{array}$ & $\begin{array}{l}\text { STRAW } \\
\text { OPEN }\end{array}$ & $P$ value \\
\hline $\begin{array}{l}\text { Pooled pre-partum cortisol } \\
\text { concentration }(\mathrm{ng} / \mathrm{ml})\end{array}$ & $30.4^{\mathrm{b}} \pm 2.2$ & $29.1^{\mathrm{b}} \pm 1.9$ & $22.9^{\mathrm{a}} \pm 1.8$ & $30.5^{\mathrm{b}} \pm 2.2$ & 0.008 \\
\hline Pawing events pre-partum & $14^{\mathrm{a}} \pm 2$ & $15^{\mathrm{a}} \pm 2$ & $6^{\mathrm{b}} \pm 1$ & $51^{c} \pm 5$ & $<0.001$ \\
\hline Nosing events pre-partum & $16.3^{\mathrm{a}} \pm 10.9$ & $168.1^{\mathrm{bc}} \pm 35.9$ & $117.1^{b} \pm 29.2$ & $199.2^{c} \pm 53.4$ & 0.031 \\
\hline $\begin{array}{l}\text { Number of posture changes } \\
\text { during farrowing }\end{array}$ & $29.4^{\mathrm{a}} \pm 3.3$ & $16.1^{\mathrm{b}} \pm 1.7$ & $12.3^{\mathrm{b}} \pm 1.4$ & $38.5^{\mathrm{c}} \pm 2.7$ & $<0.001$ \\
\hline Colostrum intake $(\mathrm{g})$ & $361.9^{b} \pm 21.9$ & $341.3^{b} \pm 26.2$ & $404.8^{\mathrm{c}} \pm 22.7$ & $325.0^{\mathrm{a}} \pm 19.5$ & 0.005 \\
\hline $\begin{array}{l}\text { Piglets saved during } \\
\text { farrowing }\end{array}$ & $0.2^{\mathrm{a}} \pm 0.1$ & $0.5^{\mathrm{b}} \pm 0.2$ & $0.3^{\mathrm{ab}} \pm 0.1$ & $1.9^{\mathrm{c}} \pm 0.9$ & $<0.001$ \\
\hline
\end{tabular}

Conclusion These findings demonstrate that both straw and hessian are utilised by crated sows during the nest-building phase prior to parturition. Results from this experiment partially supported our hypotheses. However, only straw provided the most benefit when provided in a closed crate, by reducing adverse physiological parameters that are thought to indicate sow distress in the lead up to parturition, including reducing cortisol levels, posture changes during the expulsion phase of farrowing and improving piglet survival.

Acknowledgements Supported in part by Pork CRC Limited. The authors wish to thank the technical staff involved in this project; Jessica Zemitis, Serena Barnes, Lauren Staveley. Additionally, we would like to thank honours student Alice Rainsford. 


\section{The provision of straw within a farrowing crate improves piglet survival}

\section{A McKenny ${ }^{1}$, K J Plush ${ }^{3}$, T L Nowland ${ }^{1,2}$ and W H E J van Wettere ${ }^{2}$}

${ }^{1}$ South Australian Research and Development Institute, Australia, ${ }^{2}$ The University of Adelaide, Australia, ${ }^{3}$ SunPork Group, Australia

william.vanwettere@adelaide.edu.au

Application The addition of straw to the farrowing crate for sow use pre-farrowing can be used to improve pre-weaning piglet survivability.

Introduction Farrowing crates are commonly used in pig production with the aim of reducing piglet mortality; however, such confinement prevents sows from performing natural nest building behaviours pre-partum (Lawrence et al., 1994; Wischner et al., 2009). The provision of straw to farrowing crates has demonstrated benefits, including increased sow reactivity towards piglets and maternal bonding, thereby favouring piglet survival (Herskin et al., 1998). Validation within a commercial setting is required as previous studies have occurred solely in research environments. This experiment determined the effects of different nest building materials on piglet mortality under commercial conditions. We hypothesised that the addition of a nest building substrate in a commercial setting would improve piglet survival.

Material and methods 264 Large White x Landrace multiparous sows (parity $2.9 \pm 0.1$ ) were randomly assigned to one of four treatments; sows housed in a farrowing crate with no nesting substrate (CONTROL; $n=66$ sows), sows housed in a closed combi pen that had two hessian sacks attached to the front bars via chains (HESSIAN; n=66 sows), sows housed in a closed combi pen and given $2 \mathrm{~kg}$ chopped straw daily until farrowing (STRAW CLOSED; n=64 sows), sows housed in an open combi pen and given $2 \mathrm{~kg}$ chopped straw daily until farrowing (STRAW OPEN; $\mathrm{n}=68$ sows). Piglet survival and growth were recorded and analyses conducted in SPSS (IBM SPSS Statistics for Windows, v21.0. Armonk, NY).

Results Piglets born alive and born dead were not affected by treatment (Table 1). However, there was a treatment by parity interaction $(P=0.023)$ for the number of piglets born dead. Older parity $(6+)$ CONTROL and HESSIAN sows gave birth to significantly more stillborn piglets $(1.9 \pm 0.4$ and $1.2 \pm 0.3$, respectively) than their counterparts (STRAW OPEN $0.7 \pm 0.4$ and STRAW CLOSED $0.6 \pm 0.3$, respectively). Pre- and post-foster deaths were lowest in the STRAW CLOSED treatment and highest in the STRAW OPEN treatment. Average piglet weight at $21 \mathrm{~d}$ of age was significantly lower in the STRAW OPEN treatment when compared with all other treatments.

Table 1. Production measurements in a commercial setting from piglets born to sows from four treatment groups. Data are expressed as means \pm SEM.

\begin{tabular}{|c|c|c|c|c|c|}
\hline & CONTROL & HESSIAN & $\begin{array}{l}\text { STRAW } \\
\text { CLOSED }\end{array}$ & $\begin{array}{l}\text { STRAW } \\
\text { OPEN }\end{array}$ & $P$ value \\
\hline Total born & $13.2 \pm 0.4$ & $12.6 \pm 0.4$ & $12.4 \pm 0.5$ & $12.9 \pm 0.5$ & 0.474 \\
\hline Born alive & $12.2 \pm 0.4$ & $11.8 \pm 0.4$ & $11.6 \pm 0.4$ & $12.1 \pm 0.5$ & 0.790 \\
\hline Still born & $0.7 \pm 0.1$ & $0.7 \pm 0.1$ & $0.7 \pm 0.1$ & $0.6 \pm 0.1$ & 0.851 \\
\hline Pre foster deaths & $1.3^{\mathrm{a}} \pm 0.2$ & $1.2^{\mathrm{a}} \pm 0.2$ & $0.7^{\mathrm{b}} \pm 0.2$ & $1.9^{c} \pm 0.2$ & 0.001 \\
\hline Litter size post foster & $11.5 \pm 0.1$ & $11.4 \pm 0.1$ & $11.5 \pm 0.1$ & $11.4 \pm 0.1$ & 0.794 \\
\hline Post foster deaths & $1.1^{\mathrm{a}} \pm 0.2$ & $1.1^{\mathrm{a}} \pm 0.2$ & $0.7^{\mathrm{b}} \pm 0.2$ & $1.8^{\mathrm{c}} \pm 0.2$ & 0.001 \\
\hline Number of pigs weaned & $9.4 \pm 0.3$ & $9.5 \pm 0.3$ & $9.8 \pm 0.3$ & $9.5 \pm 0.3$ & 0.689 \\
\hline Litter weight d 21(kg) & $59.5 \pm 2.0$ & $57.4 \pm 1.8$ & $62.0 \pm 2.1$ & $55.0 \pm 2.4$ & 0.137 \\
\hline Average piglet weight d $21(\mathrm{~kg})$ & $6.2^{\mathrm{a}} \pm 0.1$ & $6.1^{\mathrm{a}} \pm 0.1$ & $6.2^{\mathrm{a}} \pm 0.1$ & $5.7^{\mathrm{b}} \pm 0.1$ & 0.046 \\
\hline
\end{tabular}

${ }^{\mathrm{a}, \mathrm{b}, \mathrm{c}}$ Means within a row with different superscripts differ significantly $(P<0.05)$.

Conclusion The results from the present study highlight the importance of colostrum intake, as pre-weaning survival was highest when sows were provided straw in a closed farrowing crate. Our previous investigation showed improvements in nesting and farrowing behaviours for both STRAW and HESSIAN crated sows, but only STRAW sows demonstrated improved colostrum intake of piglets. Interestingly, in this present study the growth of piglets in the STRAW OPEN treatment was reduced and based on our previous research (McKenny et al., 2019) this could be due to the piglets ingesting less colostrum. Thus, our recommendation is to provide straw under farrowing crate conditions to maximise pre-weaning survival and growth.

Acknowledgements Supported by Pork CRC Limited. The authors wish to thank Jena Alexopoulos and Suzanne Hallett for their technical involvement in this project.

\section{References}

Herskin MS, Jensen KH and Thodberg K 1998. Applied Animal Behaviour Science, 58, 241-254.

Lawrence AB, Petherick JC, McLean KA, Deans LA, Chirnside J, Vaughan A, Clutton E and Terlouw EMC 1994. Applied Animal Behaviour Science. 39, 313-330.

McKenny LA, Plush, KJ, Nowland TL and van Wettere WHEJ 2019 Advances in Animal Biosciences. 10, s94.

Wischner D, Kemper N and Krieter J 2009. Livestock Science. 124, 1-8. 
Systematic review and meta-analysis of pre-weaning mortality for farrowing crates and pens D Glencorse $^{1}$, K J Plush ${ }^{1}$, S J Hazel ${ }^{2}$, D N D'Souza ${ }^{1}$ and M Hebert ${ }^{2}$

${ }^{1}$ SunPork Group, Australia, ${ }^{2}$ The University of Adelaide, Australia

dannielle.glencorse@sunporkfarms.com.au

Application Current farrowing pen designs result in a 16\% increase in the relative risk of piglet mortality indicating that producers will need to further refine these pen systems to minimise the negative impact on piglet survival.

Introduction The farrowing crate has maintained crushing-based piglet mortality to $10 \%$ and is believed to be the most viable housing system for piglet survival (Marchant et al., 2000). However, there is evidence that housing sows in confined systems leads to compromised welfare. Farrowing pens result in increased piglet mortality in some studies, while others have maintained rates consistent with farrowing crates (Moustsen et al., 2013). The aim of this study was to identify if farrowing accommodation influenced sow performance using a systematic review and meta-analyses approach.

Material and methods A literature search was conducted using Scopus, BIOSIS Previews, Cab Abstracts and Web of Science with 6695 articles retrieved and 21 publications included for the qualitative systematic review and quantitative meta-analysis. Each datapoint required a comparison between a farrowing crate and any variety of farrowing pen. Data was extracted from publications that allowed for the calculation of pre-weaning piglet mortality ( $\mathrm{n}=30$ ). Individual random effects meta-analyses were conducted to provide an estimate of the change in the mean relative risk of housing sows in farrowing pens in comparison with crates.

Results The qualitative analysis of data identified that $57 \%$ of studies found an increase in total piglet mortality in farrowing pens when compared with farrowing crates, $40 \%$ of studies observed a decrease and $3 \%$ of studies observed no change. The mean pre-weaning mortality for farrowing crates was 102 piglets/trial (range=7-506) which was lower than 123 piglets/trial (range $=9-590$ ) in pens. The relative risk of piglet mortality for a sow housed in a farrowing pen was $16 \%$ higher than in a farrowing crate (Figure 1).

\begin{tabular}{|c|c|c|c|c|c|c|c|}
\hline \multirow[b]{2}{*}{ Author(s) and Year } & \multicolumn{2}{|c|}{ Crate } & \multicolumn{2}{|c|}{ Pen } & & & \multirow[b]{2}{*}{ Relative Risk $[95 \%, \mathrm{Cl}]$} \\
\hline & \#Piglets & \#Died & \#Piglets & \#Died & & & \\
\hline Blackshaw et al.,1994 & 108 & 15 & 105 & 34 & & $\longmapsto$ & $0.43[0.25,0.74]$ \\
\hline Chidgey et al.,2015 & 4026 & 246 & 4677 & 478 & & 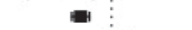 & $0.60[0.52,0.69]$ \\
\hline Cronin et al.,2000 & 856 & 150 & 706 & 109 & & $H$ & $1.13[0.91,1.42]$ \\
\hline Gu et al.,2011 & 67 & 7 & 63 & 16 & & $\longmapsto$ & $0.41[0.18,0.93]$ \\
\hline Gu et al.,2011 & 67 & 7 & 64 & 6 & & $\longmapsto$ & $1.11[0.40,3.14]$ \\
\hline IIImann et al.,2016 & 246 & 23 & 246 & 30 & & $\longmapsto-1$ & $0.77[0.46,1.28]$ \\
\hline Condous et al.,2016 & 714 & 97 & 244 & 36 & & $\mapsto-1$ & $0.92[0.65,1.31]$ \\
\hline Condous et al.,2016 & 714 & 97 & 516 & 68 & & $\mapsto-1$ & $1.03[0.77,1.38]$ \\
\hline Condous et al.,2016 & 714 & 97 & 242 & 57 & & $\mapsto-1 \vdots$ & $0.58[0.43,0.77]$ \\
\hline Condous et al.,2016 & 714 & 97 & 500 & 82 & & $1+4$ & $0.83[0.63,1.09]$ \\
\hline McGlone \& Blecha, 1987 & 106 & 31 & 97 & 9 & & 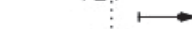 & $3.15[1.58,6.28]$ \\
\hline Collins et al.,1987 & 590 & 71 & 620 & 77 & & $1-1$ & $0.97[0.72,1.31]$ \\
\hline Cronin \& Smith,1992 & 111 & 11 & 111 & 9 & & 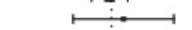 & $1.22[0.53,2.83]$ \\
\hline Cronin \& Smith, 1992 & 82 & 9 & 75 & 12 & & 0 & $0.69[0.31,1.54]$ \\
\hline Lou \& Hirnik,1994 & 269 & 76 & 285 & 64 & & $t=-1$ & $1.26[0.94,1.68]$ \\
\hline Moustsen et al.,2013 & 755 & 34 & 798 & 75 & & $\mapsto-1 \vdots$ & $0.48[0.32,0.71]$ \\
\hline Moustsen et al.,2013 & 755 & 34 & 735 & 38 & & $\longmapsto$ & $0.87[0.55,1.37]$ \\
\hline Moustsen et al.,2013 & 755 & 34 & 788 & 31 & & $\longmapsto$ & $1.14[0.71,1.84]$ \\
\hline Yun et al.,2014 & 146 & 19 & 124 & 19 & & $\longmapsto$ & $0.85[0.47,1.53]$ \\
\hline Hales et al.,2014 & 1034 & 130 & 4153 & 590 & & 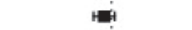 & $0.88[0.74,1.06]$ \\
\hline Hales et al.,2014 & 4181 & 506 & 3665 & 579 & & - : & $0.77[0.69,0.86]$ \\
\hline Hales et al.,2014 & 4396 & 470 & 3263 & 382 & & 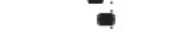 & $0.91[0.80,1.04]$ \\
\hline Hales et al.,2015 & 513 & 88 & 531 & 97 & & $\mapsto-1$ & $0.94[0.72,1.22]$ \\
\hline Hales et al.,2015 & 513 & 76 & 513 & 107 & & $1=-$ & $0.71[0.54,0.93]$ \\
\hline Ison et al.,2015 & 140 & 24 & 98 & 16 & & $\longmapsto$ & $1.05[0.59,1.87]$ \\
\hline Lambertz et al.,2015 & 704 & 80 & 691 & 89 & & $1=-1$ & $0.88[0.66,1.17]$ \\
\hline Lambertz et al.,2015 & 704 & 80 & 755 & 100 & & $1=-1$ & $0.86[0.65,1.13]$ \\
\hline Melisova et al.,2014 & 221 & 25 & 246 & 30 & & $\longmapsto$ & $0.93[0.56,1.53]$ \\
\hline Morrisson et al.,2015 & 1685 & 280 & 1670 & 332 & & $-i$ & $0.84[0.72,0.97]$ \\
\hline Payne et al.,2009 & 1716 & 118 & 1716 & 115 & & $1+1$ & $1.03[0.80,1.31]$ \\
\hline \multirow[t]{3}{*}{ RE Model } & & & & & & 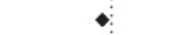 & $0.86[0.78,0.95]$ \\
\hline & & & & & $\Gamma$ & $T$ & \\
\hline & & & & & 0.05 & 0.25 & \\
\hline
\end{tabular}

Figure 1. Relative risk $(95 \% \mathrm{CI})$ of pre-weaning piglet mortality recorded for farrowing crates and pens by publication

Conclusion This was the first systematic review and meta-analyses focused on the performance of farrowing pens and crates. Post-natal piglet survival was decreased by housing sows in non-confinement farrowing accommodation. The freedom of sow movement in a farrowing pen is associated with more overlays (Oliviero et al., 2008). Thus, the continued use of farrowing crates will ensure optimum piglet survival rates.

Acknowledgements Supported in part by Pork CRC Limited (Project number 1A-117).

\section{References}

Oliviero C, Heinonen M, Valros A, Halli O and Peltoniemi OAT 2008. Animal Reproduction Science. 105, 365-377. Marchant JN, Rudd AR, Mendl MT, Broom DM, Meredith MJ, Corning S and Simmins PH 2000. Veterinary Journal. 147, 209-214.

Moustsen VA, Hales J, Lahrmann HP, Weber PM and Hansen CF 2013. Animal. 7, 648-654. 
Use of a synthetic olfactory agonist in the farrowing house

$\underline{\mathrm{R} \text { Terry }}^{1}$, T L Nowland ${ }^{1,2}$ and K J Plush ${ }^{3}$

${ }^{1}$ South Australian Research and Development Institute, Australia, ${ }^{2}$ The University of Adelaide, Australia, ${ }^{3}$ SunPork

Group, Australia

robyn.terry@sa.gov.au

Application Providing a synthetic olfactory agonist in the farrowing crate prior to parturition did not affect sow cortisol levels prior to parturition.

Introduction Farrowing has been suggested to be a time of heightened distress for sows. Appeasing pheromones are naturally secreted pheromones from the mammary glands of lactating sows that are absorbed by the piglet via the nasal cavity, stimulating the hypothalamus and amygdala regions of the brain (Morrow-Tesch and McGlone, 1990). A synthetic product that mimics such pheromones (SOA) has reduced sow aggression and stress at mixing (Plush et al., 2016). This experiment aimed to determine if sow exposure to SOA would reduce sow stress to a snout rope test (SRT) prior to farrowing, and if SOA influenced farrowing duration. It was hypothesised that the SOA would reduce sow cortisol levels in response to the SRT and reduce farrowing duration.

Material and methods A total of 47 sows were randomly allocated to treatment at farrowing shed entry with parity evenly distributed across treatments and blocks. The two treatments were: Control where no synthetic olfactory agonist was supplied ( $n=24 ; n=7$ first litter; and $n=17$ second litter sows) and; synthetic olfactory agonist (SOA; SecurePig ${ }^{\circ}$, SIGNS Laboratories, France): a SOA diffuser block hung in the creep area of each farrowing crate for a minimum of 5 days' prior to the expected farrowing date ( $n=23 ; n=6$ first litter; and $n=17$ second litter sows). Treatments were allocated into two separate farrowing rooms ensuring sows within the Control treatment were not exposed to the SOA. Prior to farrowing, a subset of sows were selected to determine if the SOA block had an effect on their ability to cope with stress (Control: $\mathrm{n}=6$; SOA: $\mathrm{n}=7$ ). A SRT was conducted 3.7 days prior to farrowing. The sow was snared with a rope for a total of 5 minutes to provide a stressful event and blood samples collected via an indwelling ear-vein catheter were analysed for cortisol concentration prior to and post- SRT. Cortisol data were sqrt transformed before analysis using a linear mixed model and time as the repeated measure. Farrowing duration $\left(\log _{10}\right)$, measured in real time from the birth of the first to last piglet, was analysed by univariate GLM with batch as a random effect and parity, treatment and their interactions as fixed effects (IBM SPSS Statistics for Windows, v24.0. Armonk, NY). All data are presented as back transformed values.

Results All sows demonstrated an immediate increase in plasma cortisol levels following the SRT however, there was no effect of treatment on changes in cortisol response to the SRT (Figure $1 ; P=0.612$ ). First litter sows exposed to SOA had a reduced farrowing duration compared to the Control (121.1 vs 382.8 mins, respectively; $P<0.0001$ ). Further, farrowing duration was reduced in first litter sows receiving SOA compared to second litter sows in both treatments (121.1 vs 184.5 and 187.9 mins, respectively; $P<0.0001)$. Farrowing duration was not significant between second litter sows.

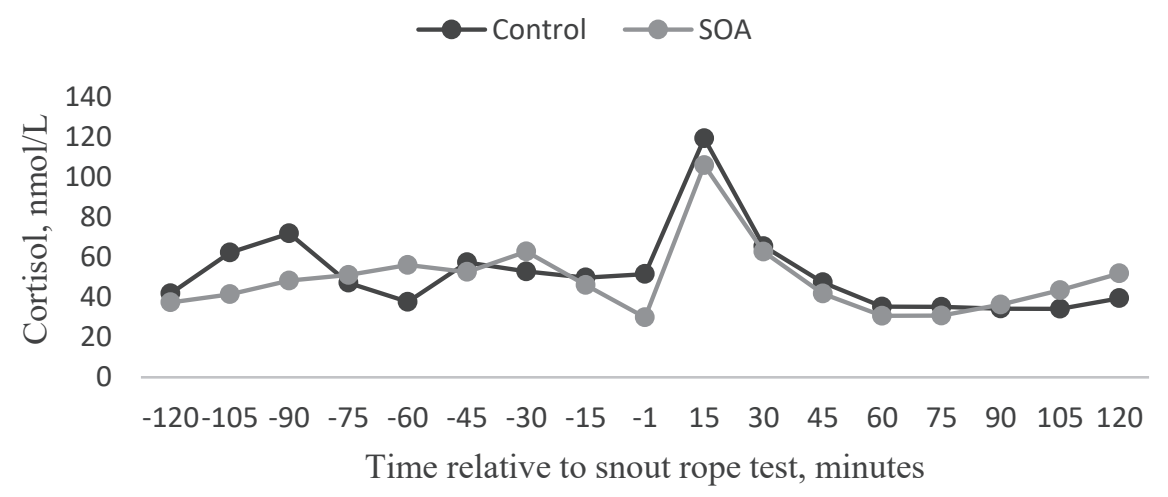

Figure 1. Sow cortisol concentration (nmol/L) 120 minutes pre- snout rope test, 1 minute prior to test and 120 minutes post- snout rope test for control and SOA treatments.

Conclusion These data indicate that the SOA can reduce farrowing duration of first litter sows. However, with such small sample sizes and the variability in the causes of extended farrowing duration, we cannot currently draw a conclusion on the effectiveness of the SOA to reduce farrowing duration or stress in pre-partum sows.

Acknowledgements Supported in part by Pork CRC Limited. We thank Jessica Zemitis, Lisa McKenny and Serena Barnes for their technical assistance in data collection.

\section{References}

Morrow-Tesch J and McGlone JJ 1990. Journal of Animal Science. 68, 3563-3571.

Plush K, Hughes P, Herde P and van Wettere W 2016. Applied Animal Behaviour Science. 185, 45-51. 


\section{Preliminary study: A novel snout cooling system in farrowing huts reduced stillbirths in free range sows during summer}

M Trezona $^{1,2}$, I Stensland ${ }^{2}$, E Loudon ${ }^{1}, \mathrm{Z} \mathrm{Cochran}^{3}$ and S Dutton ${ }^{4}$

${ }^{1}$ Pork Innovation WA Inc., Australia, ${ }^{2}$ Linley Valley Pork, Australia, ${ }^{3}$ Cool Sparky, Australia, ${ }^{4}$ University of Southern Queensland, Australia.

mtrezona@craigmostyn.com.au

Application Providing a source of cooled air in free range farrowing huts may reduce stillbirths in free range sows during summer.

Introduction In southern Western Australia (WA) free range (FR) sows, housed outdoors with free access to bedded shelter, have significant extended exposure to physiological heat stress during the summer period. Maximum daily temperatures can exceed $27^{\circ} \mathrm{C}$, the upper critical temperature for a $180 \mathrm{~kg}$ lactating sow (Farran, 1990), on more than $50 \%$ of the days during this time (January to April 2017, Mount Barker WA (BOM, 2018)) Addressing heat stress in FR sows is important for improving efficiency and welfare. High ambient temperature in the days preceding farrowing can reduce the number of live born piglets (Wegner et al., 2014). The objective of this study was to provide some relief for sows from high ambient temperatures in the days before farrowing, and the hypothesis was that a source of cooled air in the farrowing hut would positively impact upon the number of piglets born alive.

Material and methods A paddock, containing 11 individual sow farrowing huts, a water trough, and a wallow was selected as the project site on a commercial APIQ $\sqrt{ }{ }^{\circledR}$ Free Range accredited farm located in the South West of WA. All farrowing huts were of the same rectangular design, made of marine ply and positioned to face east. Huts were bedded with fresh straw for each batch. Sows, (9 to 11 per batch) were moved to the paddock approximately one week before farrowing and left to self select huts. Prototype, solar powered, refrigerated cooling units provided cooled air to farrowing huts via a $25 \mathrm{~mm}$ outlet located on the side wall near the door. Farrowing data were collected across three batches from up to four cooled huts and seven control huts per batch (January to May 2018). For cooled huts, fans initiated at an external ambient temperature of $21^{\circ} \mathrm{C}$ and cooling initiated at $23^{\circ} \mathrm{C}$. When the ambient temperature was between $23-30^{\circ} \mathrm{C}$ the air was chilled to $16-18^{\circ} \mathrm{C}$. At higher temperatures, chilled air remained about $10^{\circ} \mathrm{C}$ cooler than ambient air. Data were analysed by general ANOVA (unbalanced design) with parity as a covariate (GenStat $19^{\text {th }}$ ed, VSN International, Hemel Hempstead, UK).

Results Sows that farrowed in huts with snout cooling $(n=10)$ had a lower number of stillbirths compared to the sows that farrowed in control huts $(\mathrm{n}=19)(P=0.009)$. Consequently, the percentage of piglets born alive was higher for sows in the cooled huts $(95.6 \%)$ compared to control huts $(85.9 \%)$.

Table 1. Performance for free range sows farrowed during summer in huts with and without snout cooling.

\begin{tabular}{lcccc}
\hline & Snout cooling & Control & SED & $P$ value \\
\hline Parity & 2 & 2 & 0.426 & 0.797 \\
Total number born & 10.8 & 13.6 & 1.64 & 0.113 \\
Number born alive & 10.4 & 11.4 & 1.46 & 0.500 \\
Number of stillbirths & $0.4^{\mathrm{a}}$ & $2.1^{\mathrm{b}}$ & 0.59 & 0.009 \\
\% Born alive & $95.6^{\mathrm{a}}$ & $85.9^{\mathrm{b}}$ & 3.93 & 0.020 \\
\hline
\end{tabular}

${ }^{\mathrm{a}, \mathrm{b}}$ Means within a column with different superscripts differ significantly $(P<0.05)$; SED, standard error of difference of means.

Conclusion The likelihood of stillbirth increases with the farrowing duration and birthing interval, however there is evidence that high ambient temperature in the days immediately before farrowing can increase the number of stillbirths. Higher total numbers born tend to extend farrowing duration so can also increase the risk of stillbirths however, the results suggest that further investigations are warranted to determine if the provision of a source of cooled air in huts during the pre-farrowing period could positively impact the number of piglets born alive in FR production systems during summer.

Acknowledgements Supported in part by the Growers Group R\&D grants program administered through the WA Department of Primary Industries and Regional Development's Agribusiness Innovation Fund (GGRD-2015-0069AGSC). The assistance from staff at Craig Mostyn Farms is gratefully acknowledged. Special thank you to Hugh Payne whose ideas instigated this project.

\section{References}

BOM 2018. Bureau of Meteorology, Commonwealth of Australia, Melbourne, Australia.

Farran IG 1990. In Pig Production in Australia: 2nd Edition (Eds: Gardner JAA, Dunkin AC, Lloyd LC). Butterworths Pty Limited, Australia. pp. 112-122.

Wegner K, Lambertz C, Daş G, Reiner G and Gauly M 2014. Animal. 8, 1526-1533. 


\section{Proof of concept: Does snout cooling in farrowing huts during summer encourage free range sows to remain with piglets for longer periods during early lactation?}

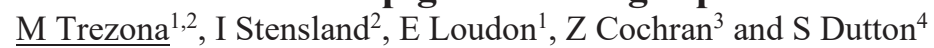

${ }^{1}$ Pork Innovation WA Inc., Australia, ${ }^{2}$ Linley Valley Pork, Australia, ${ }^{3}$ Cool Sparky, Australia, ${ }^{4}$ University of Southern Queensland, Australia.

mtrezona@craigmostyn.com.au

Application Providing a source of cooled air to free range farrowing huts in summer may encourage lactating sows to remain with piglets for longer periods during early lactation, potentially providing piglets with more suckling opportunities.

Introduction Farm records have indicated that pre-weaning mortality (PWM) in free range (FR) farrowing units is higher in summer (17\%) compared to the remainder of the year (14\%). Heat stress in lactating sows causes lower milk production and therefore reduced piglet growth during lactation (Black et al., 1993). In a FR production system sows have the ability to leave the farrowing hut, and therefore their piglets, for periods of time to seek a more comfortable environment, for example, a wallow. This may reduce the opportunities that the piglets have to suck and so impact piglet growth rate. The piglets are confined to the hut through the use of fenders for approximately one week after birth. This proof of concept study tested the hypothesis that the provision of a source of cooled air within the farrowing hut during summer, would encourage the lactating sow to remain within the hut for longer periods of time, allowing greater suckling opportunities for the piglets during early lactation.

Material and methods The project site, farrowing huts and prototype solar powered snout coolers were described by Trezona et al. (2019). In short, within a farrowing paddock there were control huts and huts fitted with an outlet that provided a source of cooled air (snout cooling) where fans initiated at an external ambient temperature of $21^{\circ} \mathrm{C}$ and cooling initiated at $23^{\circ} \mathrm{C}$. Each batch had 9 to 11 sows which were moved into the farrowing paddock one week before farrowing. Two cooled huts and two control huts per batch were video monitored during daylight hours, during the trial there was an occasion where a cooled hut and a control hut were not occupied $(n=5)$. Three batches were run between January to May 2018. Footage of pig activity was recorded between $0900-1700 \mathrm{~h}$ daily for the first $5 \mathrm{~d}$ after farrowing to determine the total time the sow spent away from the hut and the number of lactation events that occurred during the observation period. Data were analysed by general ANOVA with parity as a covariate (GenStat $19^{\text {th }}$ ed, VSN International, Hemel Hempstead, UK).

Results There were no statistical differences found, however, despite the large variation observed there was a trend $(P=$ 0.083 ) for sows in control huts to spend more time away from the hut between 0900 and $1700 \mathrm{~h}$ during the first $5 \mathrm{~d}$ after farrowing compared to sows that had farrowed in huts with snout cooling. Observations suggested that when sows returned to the hut after extended periods away suckling events occurred more frequently for a shorter duration. Preweaning mortality did not differ between cooled and control huts $(7.9 \%$ cooled, $6.9 \%$ control; $P=0.783)$.

Table 1. Average daily activity for sows and piglets between $0900-1700 \mathrm{~h}$ for the first five days after farrowing ( $\mathrm{n}=5$ ).

\begin{tabular}{lcccc}
\hline & Snout cooling & Control & SED & $P$ value \\
\hline Time sow spends outside of hut (mins/day) & 37.3 & 92.2 & 30.84 & 0.083 \\
Suckling events/day & 10.8 & 9.7 & 1.19 & 0.370 \\
\hline
\end{tabular}

Conclusion The findings suggest that the provision of cooling may encourage sows to remain in farrowing huts for longer periods during early lactation however there was little effect on the number of suckling opportunities for piglets. More replication is required to confirm the impact of providing a source of cooled air in FR farrowing huts on sow behavior and more measures are required to determine if the impact on suckling patterns will affect the growth of piglets. Extended periods between suckling may increase milk production per suckling event but decrease overall milk production, and therefore daily milk intake by the piglet is lower (Spinka et al., 1997). The review of footage also highlighted the variability between individual sows for various maternal behaviours, including time spent away from piglets and the number of suckling events per day. In confined farrowing systems this variability can be minimised, however in FR production there may be opportunities to develop strategies to manage or manipulate sow behaviours and ultimately improve piglet survivability and growth performance.

Acknowledgements Supported in part by the Growers Group R\&D grants program administered through the WA Department of Primary Industries and Regional Development's Agribusiness Innovation Fund (GGRD-2015-0069AGSC). The assistance from staff at Craig Mostyn Farms is gratefully acknowledged. Special thank you to Hugh Payne whose ideas instigated this project.

\section{References}

Black JL, Mullan BP, Lorschy ML and Giles LR 1993. Livestock Production Science. 35, 153-170.

Spinka M, Illmann G, Algers B and Stetkova Z 1997. Journal of Animal Science. 75, 1223-1228.

Trezona M, Stensland I, Loudon E, Cochran Z and Dutton S 2019. Advances in Animal Biosciences. 10, s98. 


\section{Author Index}

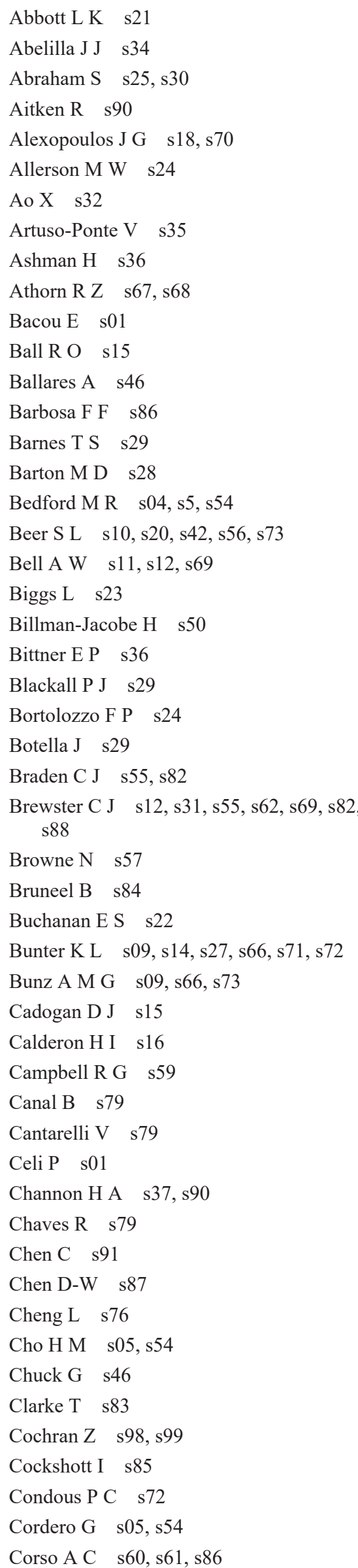

\begin{tabular}{|c|c|}
\hline Cottrell J J s11, s12, s19, s35, s69 & Hetchler B s22 \\
\hline Craig J R s19, s20, s55, s88 & Hewitt R J E s60, s61, s86 \\
\hline D’Souza D N s18, s36, s60, s61, s86, s96 & Hiendleder S s93 \\
\hline de Groot N s51, s52 & Hieu H L s35 \\
\hline De Luca V s64, s65 & Hilbrands A M s22 \\
\hline de Ruyter E M s88 & Hill I s15 \\
\hline Degroote J s03 & Hine B C s27 \\
\hline dela Cruz A R s29 & Hong J S s05, s54 \\
\hline Della Pia E A s58 & Horgan K s57 \\
\hline Delles R M s62 & Howard A J s41 \\
\hline DeRouchey J M s16, s24 & Hu J s32, s63, s76, s77 \\
\hline Doyle R E s42 & Hughes P E s92 \\
\hline Dritz S S s16, s24 & Hutchings $S$ s36 \\
\hline Dunlop R H s46 & Janni K A s22 \\
\hline Dunshea F R s11, s12, s19, s33, s36, s69 & Jenkin S s26 \\
\hline Dutton S s98, s99 & Jenkins S N s21, s53 \\
\hline Edwards A C s83 & Johnston L J s22 \\
\hline Edwards L E s42 & Johnston R s14 \\
\hline Edwards L J s50 & Jolley J Y C s37, s40, s41 \\
\hline Faba L s51 & Jordan D s30 \\
\hline Faccin J E G s24 & Jung H I s63 \\
\hline Ferguson N F s52 & Kelly J s13 \\
\hline Ford E M s12, s68, s69 & Kerssen N s08 \\
\hline Frerichs K A s57 & Kiermeier A s39, s40, s47, s48, s74, s75 \\
\hline Frugé E D s57 & Kim I H s32, s63, s76, s77 \\
\hline Furness J B s35 & Kim J C s04, s05, s44, s53, s54, s55, s75 \\
\hline Gannon N s56 & Kim Y B s05, s54 \\
\hline Gerhart A J s57 & Kim Y M s32 \\
\hline Glencorse D s18, s96 & Kind K s13 \\
\hline Goodband R D s16, s24 & Kirkwood R N s07, s17, s28 \\
\hline Gourley K M s16 & Klapish E s31 \\
\hline Grapentin J s86 & Kocher A s62 \\
\hline Greenwood E C s68, s92 & Lahaye L s59 \\
\hline Grupen C G s67 & Laird T s25 \\
\hline Guzmán-Pino S A s64, s65 & Lealiifano A K s37, s62, s90 \\
\hline $\begin{array}{l}\text { Hamilton D R s39, s41, s47, s48, s74, } \\
\text { s75 }\end{array}$ & $\begin{array}{l}\text { Lester S } \quad \text { s74 } \\
\text { Leury B J s11, s12, s69 }\end{array}$ \\
\hline Hansen E L s57 & LiY s22 \\
\hline Hansen S A s57 & Lines D S s07, s17 \\
\hline Harper J s27, s71, s72, s73 & Little S B s50 \\
\hline Hayes M s43 & Liu D s91 \\
\hline Hazel S J s96 & Liu F s10, s11, s12, s20, s69, s73, s82 \\
\hline Hebert M s96 & Lobo E T s26 \\
\hline Heim G s52 & Loudon E s33, s98, s99 \\
\hline Heller J s30 & Low W Y s28 \\
\hline Hemsworth L M s43 & Luo Y s81 \\
\hline Hemsworth P H s43 & Luo Y-H s87 \\
\hline $\begin{array}{l}\text { Henman D J s12, s20, s31, s56, s62, s69, } \\
\text { s88 }\end{array}$ & Luxford B G s09, s66 \\
\hline Heo J M s05, s54 & Macelline S P s05, s54 \\
\hline Hermesch S s08, s09, s14, s27, s66, s72 & $\begin{array}{l}\text { Mallo J J s79, s80 } \\
\text { Mansfield J P s04, s25, s44, s53, s78 }\end{array}$ \\
\hline Hernández-Jover M s30 & Maronich A s37 \\
\hline
\end{tabular}




\begin{tabular}{|c|c|c|}
\hline Marsh L s93 & Savage K s46 & Wiedemann S G s23 \\
\hline Mason M s29 & Schmeisser J s01 & Wijesiriwardana D A s19 \\
\hline Matsumoto N s46 & Segers L $\quad$ s84 & Wilkinson A R s62 \\
\hline McGahan E J s23 & Sharpe K T s22 & Woodworth J C s16, s24 \\
\hline McKenny L A s94, s95 & Sherwood D s86 & Wooten $\mathrm{H}$ s06 \\
\hline McQuade R s35 & Shi H s77 & Wu J s81 \\
\hline Meaney D s56 & Siegford J s91 & Yee $\mathrm{S} \quad \mathrm{s} 26$ \\
\hline Meers J s29 & Sinclair M s84 & Yu B s87 \\
\hline Mesas L s79 & Smith P s37, s90 & Zentek J s80 \\
\hline Meza K s84 & Smith S s10, s73 & Zhai $\mathrm{H} \quad$ s81, s87 \\
\hline Michiels J s03 & Smits R J s12, s37, s55, s69, s90 & Zhang S s32 \\
\hline Miller D W s44, s78 & Smyth D s37 & Zhao W s11, s12, s35, s69 \\
\hline Montalvo G s85 & Sol C s79, s80 & Zhu W s91 \\
\hline Monteiro A s85 & Stamatopoulos K s58 & \\
\hline Moore K L s33, s38, s83 & Staveley L M s13 & \\
\hline Morales J s85 & Steel A N s67 & \\
\hline $\begin{array}{l}\text { Morrison R S s09, s12, s14, s42, s43, } \\
\text { s66, s68, s69, s82, s88 }\end{array}$ & $\begin{array}{ll}\text { Steibel J } & \text { s91 } \\
\text { Stein H H } & \text { s34 }\end{array}$ & \\
\hline Muller M s02 & Stensland I s04, s38, s98, s99 & \\
\hline Musgrave I s41 & Sterndale S O s44, s78 & \\
\hline Nahar N s26 & Sumner J s40 & \\
\hline Nairn K s46 & Swanson A J s16 & \\
\hline Norton T s91 & Tactacan G $\quad$ s59 & \\
\hline Nowland T L s28, s94, s95, s97 & Tallaksen J E s22 & \\
\hline O’Halloran K S s20, s88 & Taylor-Pickard J A s57 & \\
\hline O'Dea M s25, s30 & Terry R s93, s97 & \\
\hline Palmieri C s29 & Tilbrook A s02 & \\
\hline Parke C s29 & Tokach M D s16, s24 & \\
\hline Pedersen N R s58 & Torok V A s $\mathbf{s} 8$ & \\
\hline Peelman L s03 & Trezona M s38, s98, s99 & \\
\hline Perez Calvo E s01 & Tritton S M s18, s60, s61, s86 & \\
\hline Phillips D s35 & Turni C s26, s29 & \\
\hline $\begin{array}{l}\text { Plush K J s07, s17, s18, s28, s42, s70, } \\
\text { s94, s95, s96, s97 }\end{array}$ & Turpin D L s04, s25, s45, s46, s49, s89 & \\
\hline $\begin{array}{l}\text { Pluske J M s49 } \\
\text { Pluske J R s04, s17, s19, s25, s44, s45, } \\
\quad \text { s49, s53, s55, s78, s89 }\end{array}$ & $\begin{array}{l}\text { Upadhaya S D s63, s77 } \\
\text { van Barneveld R J s02, s36, s60, s61 } \\
\text { van Dissel J s92 }\end{array}$ & \\
\hline Pointon A M s39, s47, s48, s74, s75 & Van Liefferinge E s03 & \\
\hline Puyalto $M \quad s 79$, s 80 & Van Noten M s03 & \\
\hline Rademacher-Heilshorn M s20 & Van Poucke M s03 & \\
\hline Rakhshandeh A s06 & van Sliedregt H s15 & \\
\hline Rakhshandeh A R s06 & $\begin{array}{l}\text { van Wettere W H E J s13, s68, s92, s94, } \\
\text { s95 }\end{array}$ & \\
\hline Ralph C R s17, s93 & Vargovic L s14, s72 & \\
\hline Ramos S H M s20 & Verlhac Trichet V s01 & \\
\hline Rayner J s92 & Vidacs E s35 & \\
\hline Reese M H s22 & Waite I S s21, s53 & \\
\hline Ren W s87 & Walker J s56 & \\
\hline Rice M s43 & Walsh M C s01, s87 & \\
\hline Rodríguez M s85 & Wang S s81 & \\
\hline Rombola L s84 & Ward S A s07 & \\
\hline Romero L F s01 & Watson K J L s23 & \\
\hline Roubos-van der Hil P s51 & Watt A s s0 & \\
\hline Roura E s02, s03, s64, s65 & Wheat L s21 & \\
\hline Sahibzada S s25, s30 & Whittaker A s93 & \\
\hline Sampaio M O s60, s61 & Wickramasuriya S S s05, s54 & \\
\hline
\end{tabular}


NOVEMBER 2019 VOLUME 10 ISSUE S1

\section{Australasian Pig Science Association}

The purpose of the Australasian Pig Science Association (Inc.) is to provide a forum for excellence in science related to all aspects of the pig industry. APSA was established in 1987 to bring together people from a range of disciplines with a primary interest in pig science.

The aim was to provide a forum for scientific discussion that would ultimately provide an impetus for further research and ultimately improve overall pig productivity. Since that time APSA has met every two years, and the conference has developed to a point where it has a world-wide reputation for science excellence and as a forum for the exchange of ideas relating to the pig industry. The conference proceedings have gained an international reputation for the quality of the symposia, reviews and short papers with all papers undergoing extensive peer review prior to acceptance.

The conference incorporates a blend of basic and applied research covering every aspect of pig science. The meeting is attended by a mix of scientists, consultants, industry support personnel and producers. APSA promotes interaction between people from different disciplines and from different countries and recognises that much of the value of a conference is the opportunity to meet new people in the industry or to renew old acquaintances. The conference is therefore held in a relaxed atmosphere with ample opportunity to socialise with other attendees.

If you would like to join or receive further information about the Australasian Pig Science Association contact:

Email: admin@apsa.asn.au

Website: www.apsa.asn.au 